\title{
Ghrelin - The Defender of Fat in the Face of Stress \\ Implications in Obesity Treatment
}

\section{Zachary R. Patterson}

A thesis submitted to the Faculty of Graduate \& Postdoctoral Affairs in partial fulfillment of the requirements of the degree of

Doctor of Philosophy

in

Neuroscience

Carleton University

Ottawa, ON

Copyright (C)

2013 - Zachary R. Patterson 


\subsection{Abstract}

Chronic activation of the stress response can lead to a number of metabolic disturbances such as obesity, metabolic syndrome, Type II diabetes and cardiovascular disease. The exact mechanisms underlying these metabolic changes are currently uncharacterized. Traditionally, many of these effects have been attributed to the increased levels of circulating glucocorticoids (cortisol in humans and corticosterone in rodents) as a function of hypothalamic pituitary adrenal (HPA) axis hyperactivity. However, recent evidence suggests that the gut derived hormone ghrelin may be a key contributor to the physiological changes generated in response to chronic stress. Ghrelin is a gut-brain peptide that promotes appetite and the accumulation of adipose tissue by encouraging the utilization of carbohydrates as a fuel source, while sparing fat tissue. Interestingly, plasma ghrelin concentrations increase in response to stressful stimuli, and remain elevated following cessation of the stressor. The present thesis was aimed at investigating the role of ghrelin in mediating stress-induced metabolic changes. In addition, this thesis explores the efficacy of potential therapeutic treatments of stress-induced metabolic disorders. Overall, the data presented in this thesis suggests that ghrelin elicits an increase in caloric intake in response to stress while promoting the utilization of carbohydrates as a fuel source. Central ghrelin signalling is required to elicit the metabolic consequences of a chronic social defeat stress. Increased ghrelin secretion in response to stress caused a significant increase in visceral adipose tissue and produced a hormonal profile indicative of obesity and metabolic syndrome. Animals lacking a functional ghrelin signalling system do not show these metabolic changes. Pharmacological blockade of ghrelin with a putative GOAT inhibitor was sufficient to reduce stress-induced ghrelin secretion and subsequent caloric intake, thereby improving the metabolic outcome of a chronic social defeat stress paradigm. 


\subsection{Co-Authors}

Albert Isaacs

Alfonso Abizaid

Elaine Waddington-Lamont

Harry MacKay

Hymie Anisman

Ian David Blum

Olga Vallegos

Rim Khazall

Robin Ducharme

Tamara Joelle Parno

Trevor Rodrigues 


\subsection{Acknowledgments}

First and foremost, I would like to thank my supervisor Dr. Alfonso Abizaid. Alfonso developed me as a scientist and lead me to where I am now, so after all I owe this to him. I would like to thank my family and friends for their patience and encouragement over the years. My colleagues and friends at the Department of Neuroscience at Carleton University created an environment that helped shape the structure of this thesis. Harry MacKay, above all others, pushed me to be the best graduate student and scientist that I could be. His relentless pursuit for perfection helped me become multidisciplinary and elevated the quality of research we conducted. 


\subsection{Dedication}

I dedicate this thesis to my beautiful girlfriend Brianne. Brianne was there to celebrate all the highs, and she supported me through all the lows. I truly could not have accomplished this without her and I owe every page of this thesis to her constant support and reassurance. 


\subsection{List of Abbreviations}

5-HIAA, 5-hydroxyindolacetic acid; 5-HT, serotonin; AC, adenylate cyclase; ACC, acetyl-CoA carboxylase; ACTH, adrenocorticotropic hormone; AgRP, agouti related peptide; AP, area postrema; ARC, arcuate nucleus of the hypothalamus; BMI, body mass index; BNST-OV, bed nucleus of the stria terminalis ; CART, cocaine-amphetamine related transcript; CCK, cholecystokinin; CeA, central nucleus of the amygdala; CMS, chronic mild stress; CORT, corticosterone; CPT- $1 \alpha$, carnitinepalmitoyle transferase- $1 \alpha$; $\mathrm{CPu}$, caudate putamen; CRF, corticotropin-releasing-factor; CSDS, chronic social defeat stress; DA, dopamine; DEX, dexamethasone; DMH, dorsomedial nucleus of the hypothalamus; DOPAC, 3,4-dihydroxy-phenylacetic acid; FAS, fatty acid synthase; GAPDH, glyceraldehyde 3-phosphate dehydrogenase; GHSR, growth hormone secretagogue receptor; GR, glucocorticoid receptor; HPA, hypothalamicpituitary-adrenal; HPLC, high-performance liquid chromatography; HVA, homovanillic acid; IL-6, interleukin 6; IP, intraperitoneal; IR, immunoreactivity; KO, knock-out; LH, lateral hypothalamus; MC4, melanocortin-4 receptor; mPFC, medial prefrontal cortex; MHPG, 3-methoxy-4- hydroxyphenylglycol; NAc, nucleus accumbens; NE, norepinephrine; NPY, neuro-peptide Y; NTS, nucleus of the solitary tract; ob-R, leptin receptor; POMC, proopiomelanocortin; PVN, paraventricular nucleus of the hypothalamus; RER, respiratory exchange ratio; $\mathrm{RF}$, restricted feeding; RIA, radioimmunoassay; RQ, respiratory quotient; RT-qPCR, real-time quantitative polymerase chain reaction; VBS, visual burrow system; VMH, ventromedial nucleus of the hypothalamus; VTA, ventral tegmentum area; SCN, suprachiasmatic nucleus of the hypothalamus; SN, substantia nigra; SSRI, selective serotonin reuptake inhibitor; TNF- $\alpha$, tumor necrosis factor- $\alpha$; UCMS, unpredictable chronic mild stress; WT, wild-type. Y2R, neuropeptide-Y receptor 2; ZT, zeitgeber; 


\section{Contents}

0.1 Abstract ........................ i

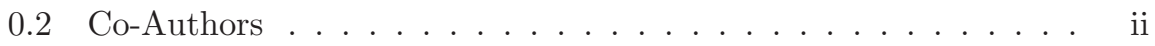

0.3 Acknowledgments . . . . . . . . . . . . . . iii

0.4 Dedication . . . . . . . . . . . . . . . iv

0.5 List of Abbreviations . . . . . . . . . . . . v

List of Figures $\quad$ x

List of Tables $\quad$ xi

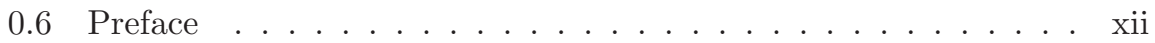

1 Stress Induced Obesity: Lessons from Rodent Models of Stress 1

1.1 Introduction . . . . . . . . . . . . . . . 1

1.2 Stress, Eating \& Energy Homeostasis . . . . . . . . . . . . 6

1.2.1 Central Regulation of Food Intake \& Energy

Balance . . . . . . . . . . . . . . . 7

1.2.2 Stress \& Autonomic Function . . . . . . . . . . . . . 9

1.2.3 Stress \& Comfort Foods: The Role of Reward . . . . . . . 10

1.2.4 Stress \& Metabolic Hormones Interact to Regulate Energy

Balance . . . . . . . . . . . . . . 11

1.3 Stress Models . . . . . . . . . . . . . . . . . . . . . 21

1.3.1 Visible Burrow System . . . . . . . . . . . . . . . . 21

1.3.2 Resident Intruder Paradigm . . . . . . . . . . . . . . . 22

1.3.3 Chronic Mild Stressors . . . . . . . . . . . . . . . . . 25

1.3.4 Physical Stressors . . . . . . . . . . . . . . 26

1.4 Factors Influencing Metabolic Outcome . . . . . . . . . . . 29

1.4.1 Predictability . . . . . . . . . . . . . . . 30

1.4 .2 Intensity . . . . . . . . . . . . . . . . 30

1.4.3 Gender Differences . . . . . . . . . . . . . . . . . . 31

1.4.4 Genetics \& Genetic Models . . . . . . . . . . . . . . . . 33

1.4.5 Conclusion . . . . . . . . . . . . . . . 34

1.5 Hypothetical Statement . . . . . . . . . . . . . . 35 
2 Altered Metabolic \& Neurochemical Responses to Chronic Unpredictable Stressors in Ghrelin Receptor-Deficient Mice $\quad 36$

2.1 Specific Aim $1 \ldots \ldots \ldots \ldots \ldots$

2.2 Introduction . . . . . . . . . . . . . . . . 37

2.3 Materials \& Methods . . . . . . . . . . . . . . . . . . . . 38

2.3 .1 Animals . . . . . . . . . . . . . . . . . . . . 38

2.3.2 Procedure . . . . . . . . . . . . . . . . . 38

2.3.3 Caloric Intake \& Body Weight . . . . . . . . . . . . . 39

2.3.4 Hormone Level Analysis . . . . . . . . . . . . . . . . . . 40

2.3.5 Brain Monoamine Analysis . . . . . . . . . . . . . . 40

2.3.6 Statistical Analysis . . . . . . . . . . . . . . . . . 41

2.4 Results . . . . . . . . . . . . . . . . . . . . 41

2.4.1 GHSR KO Mice Do Not Show the Stressor-Induced Alterations in Caloric Intake \& Body Weight Seen in GHSR WT mice . . . . . . . . . . . . . . . . . 41

2.4.2 Aminergic Neurotransmitter Concentrations are Differentially Altered by Stress in GHSR KO Mice . . . . . . . . 42

2.5 Discussion . . . . . . . . . . . . . . . . . . . . . 43

3 Central Ghrelin Signalling Mediates the Metabolic Response of C57 BL6J Male Mice to Chronic Social Defeat Stress 52

3.1 Specific Aim $2 \ldots \ldots \ldots \ldots \ldots \ldots \ldots$

3.2 Introduction . . . . . . . . . . . . . . . . . . . 53

3.3 Materials \& Methods . . . . . . . . . . . . . . . . . . 54

3.3 .1 Animals . . . . . . . . . . . . . . . 54

3.3.2 Chronic Social Defeat Stress Paradigm . . . . . . . . . . 55

3.3.3 Chronic Intracerebroventricular Delivery of Ghrelin Receptor Antagonist . . . . . . . . . . . . 55

3.3.4 Indirect Calorimetry . . . . . . . . . . . . . . . 56

3.3.5 Tissue Processing . . . . . . . . . . . . . . . . 56

3.3.6 Hormone Analysis . . . . . . . . . . . . . . . . . 56

3.3 .7 Histology . . . . . . . . . . . . . . . . . 57

3.3 .8 RT-qPCR . . . . . . . . . . . . . . 57

3.4 Results . . . . . . . . . . . . . . . . . . . 58

3.4.1 Chronic Social Defeat Stress Increases Plasma Ghrelin Concentrations \& Causes Long Lasting Changes in Caloric

Intake \& Body Weight . . . . . . . . . . . . . . 58

3.4.2 The Effects of Chronic Social Defeat on Body Weight \& Food Intake Are GHSR-1a Dependent \& Central in Origin 58

3.4.3 Chronic Social Defeat Favours the Use of Carbohydrates in WT but not in GHSR KO Mice . . . . . . . . . . 59

3.4.4 The Divergent Metabolic Consequences of Chronic Social Defeat Stress Are Exacerbated in Aged WT \& GHSR KO Mice . . . . . . . . . . . . . . . . 59

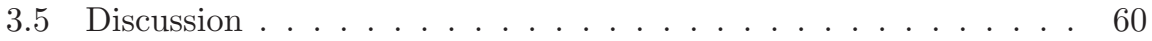


4 Interruption of Ghrelin Signalling in the PVN Increases High Fat Diet Intake \& Body Weight in Male C57BL6J Mice 72

4.1 Specific Aim 3 . . . . . . . . . . . . . . . . . . 72

4.2 Introduction . . . . . . . . . . . . . . . 73

4.3 Materials \& Methods . . . . . . . . . . . . . . . . . . . . 74

4.3 .1 Animals . . . . . . . . . . . . . . . . . 74

4.3.2 Stereotaxic Surgery - Chronic Delivery of Ghrelin Receptor Antagonist Into the Paraventricular Nucleus of the Hypothalamus . . . . . . . . . . . . . . 75

4.3.3 Chronic Social Defeat Stress Paradigm . . . . . . . . . . 75

4.3.4 Blood Plasma Collection . . . . . . . . . . . . . . . 76

4.3 .5 Statistics . . . . . . . . . . . . 76

4.4 Results. . . . . . . . . . . . . . . . 76

4.4 .1 Histology . . . . . . . . . . . . . . . 76

4.4.2 Stress Increases Caloric Intake and This Effect is Not Attenuated by GHSR Antagonism in the PVN . . . . . . . 77

4.4.3 Blocking GHSR in the PVN Increases the Intake of the High Fat Diet . . . . . . . . . . . . . 77

4.4.4 [D-Lys-3]-GHRP-6 Infusions into the PVN Does Not Influence Stress-Induced Hyperglycemia but Does Mediate Stress-Induced CORT Secretion . . . . . . . . . . . 78

4.5 Discussion . . . . . . . . . . . . . . . . . . 79

5 Ghrelin Deficient Mice Have Fewer Orexin Cells \& Reduced cFos Expression in the Mesolimbic Dopamine Pathway Under a Restricted Feeding Paradigm $\quad 85$

5.1 Specific Aim $4 \ldots \ldots \ldots \ldots \ldots$. . . . . . . . . . 85

5.2 Introduction . . . . . . . . . . . . . . . . 86

5.3 Materials \& Methods . . . . . . . . . . . . . . . . . . . . . 88

5.3 .1 Animals . . . . . . . . . . . . . . . 88

5.3.2 Restricted Feeding Paradigm . . . . . . . . . . . . . 88

5.3.3 Immunocytochemistry . . . . . . . . . . . . . . . . 88

5.3.4 Data Analysis . . . . . . . . . . . . . . . . . . . . . 89

5.4 Results . . . . . . . . . . . . . . . . . . 90

5.4.1 GHSR KO Animals Show Attenuated cFOS Expression in Major Regions of the Mesolimbic DA Pathway in Anticipation of a Scheduled Meal . . . . . . . . . . . . 90

5.4.2 Scheduled-Feeding Induces Activation of Orexin Neurons That is Reduced in GHSR KO Animals . . . . . . . . . 90

5.4.3 The Number of Orexin Neurons in the LH is Reduced in Ghrelin KO Mice . . . . . . . . . . . . . . . . . . . 90

5.5 Discussion . . . . . . . . . . . . . . . . . . . . . . 91

5.6 Conclusions . . . . . . . . . . . . . . . . . . 93 
6 Putative GOAT Inhibitor, CF 801, Prevents Stress Induced Ghrelin Secretion \& Subsequent Increases in Caloric Intake $\quad 99$

6.1 Specific Aim $5 \ldots \ldots$. . . . . . . . . . . . . . 99

6.2 Introduction . . . . . . . . . . . . . . . . . . 100

6.3 Materials \& Methods . . . . . . . . . . . . . . . . . . . . . . 101

6.3 .1 Animals . . . . . . . . . . . . . . . . . . . 101

6.3.2 CF801 Injections . . . . . . . . . . . . . . . . . 101

6.3.3 Chronic Social Defeat Stress Paradigm . . . . . . . . . . 102

6.3.4 Behavioural Analysis . . . . . . . . . . . . . . . . . . 102

6.3.5 Hormone Analysis . . . . . . . . . . . . . . . . . . . . 102

6.3 .6 Statistics . . . . . . . . . . . . . . . . 102

6.4 Results. . . . . . . . . . . . . . . . . . . 103

6.4.1 CF801 Reduces High Fat Diet Intake \& Overall Caloric

Intake . . . . . . . . . . . . . . . . . 103

6.4.2 CF801 Causes a Reduction in Body Weight in both Stressed

\& Non-Stressed Animals . . . . . . . . . . . . . . . . 104

6.4.3 Stress Tended to Increase Blood Glucose Independently

of CF801 Treatment . . . . . . . . . . . . . . . 105

6.4.4 Stress Increases Circulating Plasma Acylated Ghrelin, While

CF801 Treatment Blunts This Effect . . . . . . . . . . 105

6.4.5 Stress Increases Anxiety-like Behaviours . . . . . . . . . 106

6.5 Discussion . . . . . . . . . . . . . . . . . 106

6.6 Figures \& Tables . . . . . . . . . . . . . . . . . . . . . 110

7 General Discussion $\quad 116$

7.1 Conclusion . . . . . . . . . . . . . . . . 118

8 Appendix A - Supplemental Figures 120

9 Appendix B - Supplemental Tables 122

10 Appendix C - Copyright Documentation 124

10.1 Permission from Frontiers in Neuroendocriniology . . . . . . . . 124

10.2 Permission from European Journal of Neuroscience . . . . . . . . 124

10.3 Permission from Endocrinology . . . . . . . . . . . . . 125

10.4 Permission from Neuroscience . . . . . . . . . . . . . . 125

10.5 Co-Author Permission Statements . . . . . . . . 126 


\section{List of Figures}

1.1 Chapter 1, Figure $1 \ldots \ldots \ldots$

$2.1 \quad$ Chapter 2, Figure $1 \ldots \ldots \ldots \ldots$

2.2 Chapter 2, Figure $2 \ldots \ldots \ldots \ldots$

2.3 Chapter 2, Figure $3 \ldots \ldots \ldots$

2.4 Chapter 2, Figure $4 \ldots \ldots \ldots$

2.5 Chapter 2, Figure $5 \ldots \ldots \ldots$

2.6 Chapter 2, Figure $6 \ldots \ldots \ldots \ldots$

3.1 Chapter 3, Figure $1 \ldots \ldots \ldots \ldots$

3.2 Chapter 3, Figure $2 \ldots \ldots \ldots \ldots$

3.3 Chapter 3, Figure 3 . . . . . . . . . . . . . . . . . . 67

3.4 Chapter 3, Figure $4 \ldots \ldots$. . . . . . . . . . . . . 68

3.5 Chapter 3, Figure $5 \ldots \ldots \ldots$

3.6 Chapter 3 , Figure $6 \ldots \ldots \ldots$

3.7 Chapter 3 , Figure $7 \ldots \ldots \ldots \ldots$. . . . . . . . . . . . . .

$4.1 \quad$ Chapter 4, Figure $2 \ldots \ldots \ldots$

4.2 Chapter 4, Figure $2 \ldots \ldots \ldots \ldots$

4.3 Chapter 4, Figure $3 \ldots \ldots \ldots$

4.4 Chapter 4 , Figure $4 \ldots \ldots \ldots \ldots$

5.1 Chapter 6, Figure $1 \ldots \ldots \ldots$. . . . . . . . . . . . 94

5.2 Chapter 6, Figure $2 \ldots \ldots \ldots$

5.3 Chapter 6, Figure $3 \ldots \ldots \ldots$

5.4 Chapter 6, Figure $4 \ldots \ldots \ldots$

5.5 Chapter 6, Figure $5 \ldots \ldots \ldots \ldots$

6.1 Chapter 6, Figure 1 . . . . . . . . . . . . . . . . . 110

6.2 Chapter 6, Figure $2 \ldots \ldots \ldots 111$

6.3 Chapter 6, Figure 3 . . . . . . . . . . . . . . . . . . . . . . 112

6.4 Chapter 6, Figure $4 \ldots \ldots \ldots$

6.5 Chapter 6, Figure $5 \ldots \ldots \ldots \ldots \ldots$

6.6 Chapter 6, Figure $6 \ldots \ldots \ldots \ldots$

8.1 Chapter 3, Supplemental Figure 1 . . . . . . . . . . . . . . 121 


\section{List of Tables}

1.1 Chapter 1 , Table $1 \ldots \ldots \ldots \ldots$

2.1 Chapter 2, Table $1 \ldots \ldots \ldots \ldots \ldots \ldots$

9.1 Chapter 3, Supplemental Table 1 . . . . . . . . . . . . . . 123 


\subsection{Preface}

It is now generally accepted that in westernized societies, obesity has reached epidemic proportions. To some medical professionals, obesity is considered the leading preventable cause of death worldwide. More worrisome is the fact that the prevalence of obesity has drastically increased over the past 30 years, where $\approx 10 \%$ of the population was considered overweight in the 1970's, a number that has now risen to over 35\% in the United States (Flegal et al., 2010, 2012). This estimate is accompanied by a wealth of statistics demonstrating that individuals who are overweight or obese have a much higher susceptibility to disease such as Type II diabetes, cardiovascular disease and metabolic syndrome. In fact, some studies show that life expectancy decreases significantly as a function of weight gain, and that individuals suffering from obesity die up to a decade sooner than those of a normal weight (Peeters et al., 2003). For this reason, obesity is one of the most pressing health issues seen in the twenty-first century.

It is clear that there is a strong genetic predisposition to obesity and metabolic disorders. Nevertheless, environmental and social factors influence amounts of food consumed or energy spent and ultimately impact phenotype. Arguably, stress may be the most important environmental factor affecting metabolism and feeding behaviour to promote obesity. Indeed, there is an association between adiposity, particularly abdominal adiposity, and psychological distress (Räikkönen et al., 1996; Chrousos and Gold, 1998; Björntorp, 2001). Furthermore, and in parallel with the increasing rates of obesity, subjects from industrialized communities report higher levels of workplace stress and sleep deprivation in recent years relative to the past (Björntorp, 2001; Caruso, 2006; Grosch et al., 2006). The prevalence of chronic stressors, regardless of their origin, in combination with a secular trend in modern societies to increase food intake and decrease physical activity fuels the current obesity epidemic. In turn, the rising incidence of obesity is detrimental to health care as it exerts a financial burden to taxpayers while saturating medical services that would otherwise be available. The interaction between neural circuits involved in regulating energy homeostasis and body composition with the physiological responses to stressors has therefore become pertinent and clinically relevant. The present thesis aims to investigate the influence of stress-related hormones, while emphasizing the role of ghrelin, on energy homeostasis and adiposity, to ultimately shed light on the link between stress and obesity. 


\title{
Chapter 1
}

\section{Stress Induced Obesity: Lessons from Rodent Models of Stress}

\author{
Z.R. Patterson \& A. Abizaid \\ Frontiers in Neuroscience, 2013 \\ 7:130. doi: $10.3389 /$ fnins. 2013.00130
}

\section{$1.1 \quad$ Introduction}

Stress is defined as the behavioural and physiological changes generated in the face of, or in anticipation of, a perceived threat. These changes generated in response to a stressor represent an organisms attempt at coordinating processes to maintain a steady internal environment, or homeostasis (Cannon, 1929). Allostasis is a term used to describe the level of activity required to maintain physiological stability during times of change (Goldstein and McEwen, 2002; McEwen, 1998, 2000). Within the context of stress, allostasis refers to the metabolic processes required to maintain homeostatic balance while dealing with a stressor. The physiological and behavioural responses to stress are necessary for survival. An organism's response to a stressor involves the activation of the sympathetic nervous system and recruitment of the hypothalamic-pituitaryadrenal (HPA) axis, with particular attention paid to allostasis and homeostatic preservation (McEwen, 2007; Ulrich-Lai and Herman, 2009). The noradrenergic system, which drives sympathetic nervous system activation, originates predominantly in the locus coeruleus (LC) and the nucleus of the solitary tractus (NTS) where small populations of neurons send widespread projections that innervate nearly the entire central nervous system (CNS). Therefore, the noradrenergic system possesses ubiquitous control over a variety of physiological processes in the presence of elevated NE, as is the case during stress. It has been shown that 
$\mathrm{NE}$ can both excite and inhibit several brain regions critically involved in the response to stress, but furthermore NE can influence the sensitivity of other afferent signals in response to stress, thereby enhancing synaptic transmission at specific target sites (Woodward et al., 1991). Encountering a stressful stimuli, whether real or perceived, causes a direct activation of hindbrain noradrenergic neurons (Aston-Jones et al., 1991; Rasmussen et al., 1986). Stress-induced NE release has been shown, both electrophysiologically and neurochemically, to occur in a number of limbic forebrain structures associated with the behavioural, cognitive, affective and neuroendocrine changes in response to a wide array of rodent stressors (Abercrombie and Jacobs, 1987; Cecchi et al., 2002; Morilak et al., 1987a,b; Pacak et al., 1995; Page et al., 1992; Svensson, 1987; Valentino et al., 1993). Amongst other things, NE release in limbic forebrain structures following acute stress has been shown to mediate the anxiogenic responses to stress, and administration $\alpha_{1}$ and $\beta_{1}$-adrenergic receptor antagonists attenuates stress-induced anxiety (Cecchi et al., 2002). Interestingly, the paraventricular nucleus of the hypothalamus (PVN) receives dense noradrenergic and adrenergic projections from the hindbrain, as well as catecholaminergic projections from limbic structures, such as the bed nucleus of the stria terminalis, which serves to integrate afferent sensory information associated with stress (Sawchenko and Swanson, 1983; Cunningham and Sawchenko, 1988; Cunningham et al., 1990). Neurons in the PVN have been shown to express multiple adrenoreceptor subtypes, such as $\alpha_{1}, \alpha_{1 B}, \alpha_{2}$ and $\beta_{1}$-adrenergic receptors (Plotsky, 1987; Calogero et al., 1988; Kiss and Aguilera, 1992; Whitnall et al., 1993; McCune et al., 1993; Pieribone et al., 1994; Day et al., 1997), rendering them viable targets for sympathetic input in response to stress.

The PVN is known to be a site of origin for central corticotropin releasing factor $(\mathrm{CRF})$ and, given its adrenergic receptor expression profile, is thought to be a point of contact between the sympathetic nervous system and the HPA axis (Vale et al., 1981; Liposits et al., 1986). The PVN can be divided into distinct subnuclei - magnocellular and parvocellular nuclei - each with unique phenotypic characteristics and neuropeptide compositions (Kiss et al., 1991; van den Pol, 1982). The neurons containing CRF are most concentrated in the medial parvocellular subdivision of the PVN, and these neurons send projections predominantly to the external layer of the median eminence where they influence adrenocorticotropic hormone $(\mathrm{ACTH})$ release. Krieger and Krieger (1970) reported that administration of NE into the PVN stimulated activation of the HPA axis, an effect thought to be mediated by $\alpha_{1}$ and $\alpha_{2}$-adrenergic receptors (Leibowitz et al., 1989; Ganong et al., 1982; Kiss and Aguilera, 1992; Saphier and Feldman, 1989; Daniels et al., 1993). Thus, afferent NE projections to the parvocellular division of the PVN stimulates these CRF-containing somatotrophs which, in turn leads to the synthesis and release of CRF into the portal hypophyseal circulation neighbouring the anterior pituitary (Antoni, 1986). Corticotrophic cells in the anterior pituitary gland synthesize and express high-affinity membrane-bound CRF receptors (Aguilera et al., 1987; De Souza and Kuhar, 1986). Once secreted into circulation, CRF binds to and activates $\mathrm{CRF}$ receptors on cotricotrophs of the anterior pituitary, initiating 
adenylate cyclase and cyclic adenosine monophosphate dependent protein kinase signalling pathways, subsequently stimulating the release of ACTH (Aguilera et al., 1986). ACTH is one of several gene products of the precursor proopiomelanocortin (POMC) gene transcribed in the pituitary. When released into the general circulation, ACTH stimulates the release of glucocorticoids (cortisol in humans and corticosterone in rodents) from the adrenal cortex via activation of the MC2 receptors (type 2 melanocortin receptor). The $\mathrm{MC} 2$ receptor is a 7-transmembrane spanning G-coupled protein receptor that is densely expressed on adrenocortical cells of the zona fasciculata in the adrenal cortex. Similar to the effects of CRF on pituitary CRF receptors, ACTH binds to MC2 receptors in the adrenal cortex to activate adenyl cyclase and increase intracellular levels of cyclic AMP (Haynes, 1958; Butcher et al., 1968). Elevated levels of cyclic AMP in turn causes a rapid increase in the level of cholesterol in proximity of the inner mitochondrial membrane, providing the basis for steroidogenesis. Furthermore, ACTH is capable of elevating the levels steroid hydroxylases, $\mathrm{P} 450_{s c c}$ and $11 \beta$-hydroxylase for example, which are responsible for synthesizing steroid hormones (Zuber et al., 1985; Kramer et al., 1982), thus exerting control over the long-term synthesis of glucocorticoids (Hanukoglu et al., 1990).

Glucocorticoid release from the adrenal glands is the final output stage of the HPA axis, which represents, amongst other things, an organism's attempt to mobilize energy stores to fuel the brain, skeletal and cardiac muscles. The primary glucocorticoids secreted in response to stress are cortisol and corticosterone in humans and rodents, respectively. Once secreted into circulation, glucocorticoids are capable of binding to glucocorticoid receptors (GR's), which are expressed in almost every cell in the mammalian body. Unoccupied GR's reside in the cytoplasm of cells where they exist as complexes with several heat shock proteins (hsp90 \& hsp70) (Pratt et al., 2006). Once bound by glucocorticoids (i.e. cortisol or corticosterone), heat shock proteins dissociate and the receptor-ligand complex migrates to the nucleus to influence gene transcription controlling many physiological processes (e.g. development, metabolism and immune function) (Buckingham, 2006; Hayashi et al., 2004). In many tissues, glucocorticoids serve an opposing function to insulin's attempt at storing energy, in part by suppressing insulin secretion (Dinneen et al., 1993). By inducing a transient state of insulin resistance, glucocorticoids restrain hepatic glucose production and regulate glucose delivery to peripheral tissue thereby producing an acute state of hyperglycemia (Rizza et al., 1982; Mangos et al., 2000). The peripheral tissue involved in the stress response use the acute hyperglycemic environment to meet the energetic demands posed by the stressor.

Due to the catabolic nature of glucocorticoids, it is important that activation of the HPA axis and subsequent release of glucocorticoids is limited. As such, this system is under the control of a finely tuned negative feedback loop, wherein elevated circulating glucocorticoids travel back to the hypothalamus (Sapolsky et al., 1984, 2000), medial prefrontal cortex (Diorio et al., 1993; Radley, 2008), hippocampus Herman et al. (1992) and pituitary gland (Mahmoud et al., 1984; Cole et al., 2000) to inhibit further stimulation of the HPA axis (McEwen, 2007; Ulrich-Lai and Herman, 2009). At the level of the hypothalamus, there are nu- 
merous regions that send projections directly to the medial parvocellular region of the PVN, perhaps serving as relay stations for limbic structures modulating stress circuitry. Of these hypothalamic nuclei, the medial preoptic area and dorsomedial nucleus (DMH) have gained considerable interest for their abilities to directly inhibit PVN neurons (Boudaba et al., 1996). Currently it is unclear exactly which structures project to these relay stations in attempt to dampen any further activation of the HPA axis, however it is clear these nuclei are implicated, as lesions to these areas leads to hyper-activation of the HPA axis (Herman et al., 2003). In addition to hypothalamic relay stations, direct glucocorticoid exposure has been shown to cause rapid suppression of excitatory synaptic inputs to CRF neurons within the PVN (Di et al., 2003), an effect that is thought to be mediated by the inhibitory action of endocannabinoids on upstream glutamate neurons (Di et al., 2005).

At the level of the hippocampus, glucocorticoid feedback has been shown to suppress any further activation of the HPA axis. The hippocampus expresses high levels of both GR's and MR's (Herman, 1993; Aronsson, 1988; Arriza et al., 1988) and hippocampal stimulation, for example, has been shown to decrease glucocorticoid production and secretion in both rodents and humans (Dunn and Orr, 1984; Rubin et al., 1966). In contrast, hippocampalectomy or excitotoxic hippocampal lesions have been shown to cause hyper-activation of the HPA axis as measured by excessive glucocorticoid, CRH and/or ACTH production (Fendler et al., 1961; Knigge, 1961; Knigge and Hays, 1963; Sapolsky et al., 1984; Herman et al., 1995, 1992). Others have demonstrated that glucocorticoid signalling in the hippocampus increases excitatory post-synaptic potentials via modulation of membrane-bound mineralocorticoid receptors (Olijslagers et al., 2008). Rapid excitation of efferent hippocampal neurons, in turn, leads to activation of inhibitory GABAergic input onto the neuroendocrine PVN neurons (Boudaba et al., 1996; Herman et al., 2002) and is therefore implicated in the negative feedback regulation of the HPA axis (Furay et al., 2008). However, there appears to be regional specificity when it comes to hippocampal regulation of HPA axis function. For example, Herman et al. (1995) used a sequential lesion approach to demonstrate that the inhibitory effects of the hippocampus on stress-induced corticosterone secretion is restricted to subpopulation of neurons located in the $\mathrm{CA} 1$ region of the hippocampus.

The medial prefrontal cortex (mPFC) is also responsive to stress-induced secretion of glucocorticoids and is capable of directly and/or indirectly modulating the activity of the secretory PVN neurons (Radley, 2008). For example, the prelimbic cortex, a subregion of the mPFC, projects directly to the PVN (Hurley et al., 1991; Sesack et al., 1989) and the infralmibic cortex projects extensively to the bed nucleus of the stria terminalis, medial and central amygdala and the NTS (Hurley et al., 1991; Sesack et al., 1989; Takagishi and Chiba, 1991), all of which participate in the stress response. Lesions of the mPFC stimulates $\mathrm{ACTH}$ and glucocorticoid production, as well as c-Fos immunoreactivity in the PVN (Diorio et al., 1993; Figueiredo et al., 2003a,b). In contrast, injections of corticosterone into the $\mathrm{mPFC}$ results in significant reductions of CRF and ACTH release following restraint stress in rats (Diorio et al., 1993). Together, 
these data suggest a prominent role for the $\mathrm{mPFC}$ in the regulation of HPA axis activity. After answering the energetic demand posed by a stressor, the negative feedback loop of the HPA axis ensures an efficient return to homeostatic balance following cessation of the stressor and it is therefore considered a mediator of allostasis.

The acute response to stress is one that is both necessary and beneficial because it mediates the behavioural and physiological adaptations necessary for homeostatic maintenance under stressful conditions. Protracted stressors, on the other hand, lead to an excessive energetic burden resulting in long lasting physiological changes, a process referred to as allostatic overload (Goldstein and McEwen, 2002; McEwen, 2004). Allostatic overload occurs during a protracted stressor because the cost of adaptation requires the prolonged activation of physiological systems involved in allostasis. Increasing allostatic load eventually leads to the depletion of fuel resources and wear and tear on physiological systems, a process that underlies many of the long-term health consequences of stress (Goldstein and McEwen, 2002). Failure to adjust to the energetic demands posed by this overload, or failure to reduce the stressor itself, may lead to negative health consequences, both metabolic and psychiatric (Black, 2006; Frasure-Smith and Lespérance, 2005; McEwen, 2007). Homeostatic systems predominantly use a series of negative feedback loops, which serve to regulate the internal environment. The aforementioned negative feedback loop of the HPA axis is one system involved in the maintenance of homeostasis following a stressor. Chronic dysregulation of homeostasis by increasing allostatic load has been shown to create tolerance within the HPA axis negative feedback system, ultimately leading to a pathological state as a consequence of HPA axis hyperactivity (Hennessy and Levine, 1977). Tolerance, in the context of the HPA axis negative feedback, refers to a decreased response to circulating glucocorticoids overtime after multiple exposures to a homotypic stressor. It is well known that GR's in limbic structures, such as the hippocampus, are sensitive to high levels of circulating glucocorticoids during times of stress, however chronic stress has been shown to reduce both GR mRNA and protein levels (Sapolsky et al., 1984; Herman et al., 1995; Mizoguchi et al., 2003). Downregulation of GR's at sites involved in the negative feedback loop of the HPA axis may lead to an attenuation of glucocorticoid negative feedback. For example, Mizoguchi et al. (2003) demonstrated the dexamethasone (DEX) infusions into the PFC and/or hippocampus of chronically stress rats was unable to suppress further activation of the HPA axis. Furthermore, systemic injections of DEX in chronically stressed rats showed a significant reduction in the suppressive actions of DEX (Mizoguchi et al., 2003). Given that the higher-order structures involved in regulating HPA axis activity, such as the hippocampus and mPFC, are implicated in the pathogenesis of stress related disorders (e.g. depression \& obesity), these data suggest a role of disrupted feedback mechanisms in these processes.

Chronic stress, in combination with increased availability of foods high in calories creates an environment conducive to the development of obesity and other metabolic disturbances. The interaction between neural circuits involved in regulating energy homeostasis and body composition with the physiological 
responses to stressors has therefore become pertinent and clinically relevant.

\section{$1.2 \quad$ Stress, Eating \& Energy Homeostasis}

One of the most notable physiological disturbances seen in animal models of chronic stress is an increase in metabolic rate resulting in the inappropriate breakdown of lean body mass and a conservation of fat stores (Depke et al., 2008). Basal metabolic rate stands as a reference point for which we gauge the level of metabolism necessary to sustain life. Therefore, instability of ones metabolic rate poses a threat to energy homeostasis and eventually leads to changes in body composition in the long term. The neural mechanisms that are recruited by the stress response to mediate these physiological changes are complex and involve a number of circuits that include hypothalamic networks regulating energy balance, brain stem networks regulating visceral function, sympathetic and parasympathetic mechanisms, midbrain nuclei involved in reward and arousal, and corticolimbic structures that mediate emotional and cognitive aspects related to both stress and food intake. Furthermore, stress produces dramatic endocrine and neuroendocrine responses that in themselves affect energy balance and food intake. Among these, the best-characterized responses are the activation of the sympathetic nervous system and that of the HPA axis. The former is elicited to generate rapid physiological responses including increased heart rate and respiration, glucose uptake in muscle and decreased visceral function, all important for generating a fight or flight response. Subsequent activation of the HPA axis, as previously discussed, represents a hormonal cascade that culminates in the secretion of glucocorticoids. These steroid hormones have a multitude of effects, both in the periphery and the brain that among other things alter metabolism, promote appetite and increase locomotor activities associated with food seeking behaviors in rodents (McEwen, 2004).

Numerous studies have shown that stress modulates metabolism, but these effects are not always consistent and can range from marked decreases in both body weight and food intake, to increases in body weight and calorie consumption (Dallman et al., 2005; Depke et al., 2008; Nieuwenhuizen and Rutters, 2008). For example, acute psychogenic or systemic stressors results in a rapid decline of food intake and the successive breakdown of nutrient stores (Depke et al., 2008). Similarly, exposure to chronic stressors can also lead to decrease body weight and food intake (Torres and Nowson, 2007; Depke et al., 2008). While these animals appear to lose weight, they do so by using nutrients derived from tissues other than fat in spite of decreasing their body weight (Akana et al., 1999; Depke et al., 2008). Metabolically, these animals show altered glucose regulation, an early indicator of insulin resistance (Black, 2006).

Interestingly, repeated exposure to psychogenic stressors, in particular social stressors, generates increases in body weight, adiposity, and the intake of high calorie "comfort" meals in a number of species including non-human and human primates (Pecoraro et al., 2004; Dallman et al., 2005; Tamashiro and Hegeman, 2007; Coccurello et al., 2008; O'Connor et al., 2008; Wilson et al., 2008). These 
data are of particular relevance considering that in human populations stress is highly associated with social interactions (Tamashiro and Hegeman, 2007; Tamashiro, 2007; Michaud et al., 2008; O'Connor et al., 2008; Wilson et al., 2008). The mechanisms underlying metabolic changes in response to social stressors have commonly been associated with the negative consequences of repeated stimulation of the HPA axis (Anisman and Matheson, 2005). In the case of obesity, elevated levels of glucocorticoids appear to be associated with abdominal adiposity (Black, 2006). Indeed, clinical conditions characterized by high cortisol levels, as seen in patients with Cushing's or Prader-Willi syndrome, are also associated with abdominal obesity and type II diabetes (Nieuwenhuizen and Rutters, 2008; Weaver, 2008). In contrast, removal of the adrenal glands in experimental animals results in decreased body weight and food intake that can be restored by corticosterone replacement (Saito and Bray, 1984; Pralong et al., 1993; Makimura et al., 2000).

Finally stress also affects psychological processes that could lead to the preference of foods that have comfort value. For instance, some stressors have been associated with a loss of control over food intake and an increased drive to eat highly palatable non-nutritious foods (Groesz et al., 2012). This active seeking of highly palatable foods is thought to be a form of self-medication for both humans and rodents, wherein HPA axis activation is down regulated in response to the rewarding properties of such foods ultimately creating a preference, and potentially cravings, for these foods (Dallman and Pecoraro, 2003; Dallman et al., 2005; Pecoraro et al., 2004; Tomiyama et al., 2011). Interestingly, while all other stressors are known to stimulate the HPA axis, there is a documented, bi-directional effect on food intake. Physical stress, for example, may cause a reduction in food intake (Popper et al., 1989) while psychological stress can increase or decrease food intake (Huhman, 2006).

\subsubsection{Central Regulation of Food Intake \& Energy Balance}

To understand how stress influences metabolism and feeding we first need to consider how feeding and metabolism are regulated and then how stress can influence these mechanisms. Within the brain there exists a very complex organization of nuclei that collectively control food intake and energy homeostasis. In order to do so, these circuits must first obtain all relevant information from the periphery before mounting an appropriate response. Metabolic and endocrine signals from the periphery communicate with the brain primarily through humoral signals in circulation. Peripheral tissues such as white adipose tissue, gastrointestinal tract, thyroid and adrenal glands, skeletal muscles and reproductive organs are all capable of using hormones to communicate their metabolic states with the central nervous system (Bray, 2000; Havel, 2001). There are several peripherally circulating hormones that an organism uses to convey metabolic information to the brain. Each of these hormones has a unique ability to convey specific pieces of metabolic information from the periphery and they function in concert to regulate metabolism, however only a subset of these 
hormones will be discussed in the present thesis (see below, section 1.2.4).

Traditionally, the hypothalamus is considered the focal point for processing and integrating both afferent and efferent metabolic information (Elmquist, 2000; Schwartz et al., 2000; Dhillo and Bloom, 2001; Cummings and Shannon, 2003). Within the hypothalamus, these nuclei can be roughly divided into two distinct groups based on their tendencies to promote (lateral hypothalamus) or inhibit food intake (ventromedial nucleus and paraventricular nucleus; VMH and PVN respectively). Furthermore, at the base of the hypothalamus the arcuate nucleus (ARC) lies within an area that is less protected by the blood brain barrier and is thus capable of detecting peripherally circulating metabolic signals (Ciofi, 2011; Morita and Miyata, 2012, 2013). With this real estate, and the expression of both orexigenic and anorectic peptides, the ARC is thought to play an intimate role in the regulation of food intake and energy homeostasis (Schwartz et al., 2000; Cone et al., 2001; Cowley et al., 2001, 2003). The influence of the ARC on feeding behaviours is mediated by a sub-set of firstorder neurons that either co-express the orexigenic transcripts neuropeptide $\mathrm{Y}$ (NPY) and agouti-related peptide (AgRP), or the anorectic transcripts proopiomelanocortin (POMC) and cocaine-amphetamine related transcripts (CART). NPY and AgRP are two potent orexigenic peptides found predominantly in the ARC and whose expression and secretions are mediated through impairments in energy balance such as depletions of body fat stores, reductions in circulating glucose and/or altered metabolic hormone signalling in the brain (White and Kershaw, 1990; Kalra et al., 1991; Wilding et al., 1993; Schwartz et al., 2000). While both NPY and AgRP are potent orexigenic peptides, their mechanisms of action are fundamentally different. NPY, for example, responds to circulating humoral signals of energy deficiencies by increasing the rate of firing and secreting NPY which binds to and stimulates NPY $\mathrm{Y}_{1}, \mathrm{Y}_{2}$ and $\mathrm{Y}_{5}$ receptors (Gerald et al., 1996; Herzog et al., 1993; Parker et al., 2002). In contrast, when stimulated by a negative energy balance, AgRP neurons increase their firing rate and promote food intake through the inhibition of the anorectic melanocortin $\mathrm{MC}_{3}$ and $\mathrm{MC}_{4}$ receptors in the hypothalamus (Ollmann et al., 1997). POMC and CART are also major regulators of energy balance, however, in contrast to NPY and AgRP, they produce a potent and long lasting anorexigenic effect (Kristensen et al., 1998; Hagan et al., 1999; Larsen et al., 2000; Cowley et al., 2001). The expression and secretions of POMC and CART are mediated by signals of an energy surplus such as overfeeding and increases in body weight (Hagan et al., 1999). NPY/AgRP and POMC/CART neurons are profoundly interconnected and differentially regulated by other hypothalamic nuclei involved in energy homeostasis (Broberger et al., 1997; Parker and Herzog, 1999). Recently, it has been shown that selective activation of NPY/AgRP neurons in the ARC causes a potent suppression of POMC/CART neuronal activity, and a subsequent increase in long-term food intake (Atasoy et al., 2012). Activation of NPY/AgRP neurons by peripherally circulating orexigenic signals (e.g. ghrelin) increases the firing rate of GABAergic projections that synapse with neighbouring POMC/CART neurons, thereby inhibiting their anorectic counterparts (Cowley et al., 2003). In contrast, circulating anorectic signals (e.g. 
insulin and leptin) are capable of suppressing the activation of NPY/AgRP neurons through interactions with their respective receptors (Schwartz et al., 2000; Crowley et al., 2002). Thus, in addition to being influenced by peripheral signals indicating energy availability, NPY/AgRP neurons are capable of directly regulating activity of other neuronal subpopulations involved in food intake (Wahlestedt et al., 1986; Broberger et al., 1997; Parker and Herzog, 1999; Cowley et al., 2001; Atasoy et al., 2012). Furthermore, satiety centres such as the VMH have been shown to send excitatory inputs onto POMC/CART neurons, providing a more complex inhibition of food intake (Sternson et al., 2005). Interestingly, the excitatory glutamatergic neurons in the VMH express CRF receptors (Chalmers et al., 1995) and are thus stimulated by stress-induced CRF secretion, as well as the structurally related urocortin peptides (Chen et al., 2010; Kuperman and Issler, 2010). Once stimulated, these neurons project from the $\mathrm{VMH}$ to arcuate POMC/CART neurons and therefore initiate an anorectic signalling cascade downstream of the VMH (Chen et al., 2010). In contrast, NPY/AgRP neurons are regulated by weak inhibitory inputs from within the ARC but do not respond to the VMH (Sternson et al., 2005) and are not controlled by anorectic signals.

Peripheral signals ascending via the third ventricle and/or the vagus nerve are capable of directly and/or indirectly updating the first order arcuate neurons with information pertaining to the organism's nutritional status. Once stimulated, these first order NPY/AgRP and POMC/CART neurons project to second order hypothalamic centres located predominantly in the PVN, LH, and brain stem nuclei (Clark et al., 1984; Ramos et al., 2005; Sternson et al., 2005; Palmiter, 2007; Atasoy et al., 2012; Wu et al., 2012) to regulate meal frequency (Marín Bivens et al., 1998) and meal size, respectively (Kalra et al., 1988). NPY/AgRP neurons, for example, project directly to the PVN where they are capable of producing prolonged inhibitory post-synaptic currents and a robust feeding bout (Atasoy et al., 2012). In addition, NPY/AgRP neurons project to the LH where they serve to promote the secretion of other orexigenic transcripts such as hypocretin/orexin in response to hunger signals (Cone et al., 2001). POMC/CART neurons, on the other hand, are capable of down regulating activity in these same areas. POMC/CART neurons project to the LH and PVN to reduce food intake and increase energy expenditure, respectively (Cone et al., 2001; Cowley et al., 2001).

\subsubsection{Stress \& Autonomic Function}

Integration of circulating metabolic and stress signals also occurs in several brain stem nuclei that are part of the autonomic and enteric nervous system (Berthoud et al., 2006). While the hypothalamus gathers most of the attention when discussing the integration and processing of peripheral metabolic signals, some argue that the brain stem's role is equally as important (Berthoud, 2002). As such, these nuclei are critical for the responses that follow the activation of the stress axis by psychogenic, metabolic, or immunological challenges (Smith and Vale, 2006). Within the brain stem, the nucleus of the solitary tract (NTS) 
is the main target for information ascending via the vagus nerve and the enteric nervous system. The NTS contains noradrenergic neurons that modulate the activity of hypothalamic and limbic structures implicated in feeding, including the PVN and central nucleus of the amygdala (CeA) (Treece et al., 2000; Grill and Kaplan, 2001, 2002). Another brain stem region important for the regulation of food intake and stress is area postrema (AP). The AP lies outside the blood brain barrier and is sensitive to blood born nutritional signals including hormones like leptin, insulin, cholecytokinin (CCK) and ghrelin, as well as being sensitive to changes in circulating levels of glucose. Signals are relayed from the AP to the NTS where they are integrated with ascending visceral signals, as well as to the PVN where they may elicit feeding and autonomic responses (Ellenberger and Feldman, 1990; Woulfe et al., 1990; Ferguson, 1991; Castañeyra Perdomo et al., 1992; Díaz-Regueira and Anadón, 1992; Cai et al., 1996). In addition to the regulation of feeding responses, the brain stem plays a key role in the generation of the autonomic responses, including increases in blood pressure, respiration, hepatic glucose production, vasoconstriction, lipolysis, and thermogenesis (Bamshad et al., 1998; Krukoff, 1998; Dunn et al., 2004).

Given these roles, it is not surprising that noradrenergic cells in the brain stem are affected by chronic stress. Indeed, many of the effects of chronic stress on this system are controlled by medications that block noradrenergic receptors (i.e. $\beta$-blockers) (Grassi, 2007). Moreover, NPY released from sympathetic cells into peripheral circulation may be critical for the adipogenic effects of stress, primarily by acting directly on NPY-Y2 receptors located on adipocytes (Kuo et al., 2007).

\subsubsection{Stress \& Comfort Foods: The Role of Reward}

The midbrain contains a number of cell groups implicated in brain function underlying reward seeking, learning and memory, affective states and the generation of locomotor responses. The ventral tegmentum area (VTA) has reemerged as an important structure in the modulation of food intake (Simerly, 2006). Dopamine (DA) cells within this region project to the striatum, prefrontal cortex, hippocampus and amygdala (Margolis et al., 2006; Fields et al., 2007; Robinson et al., 2007) where they produce behaviours to get rewards such as food and sex, or behaviours to escape aversive stimuli (Wang et al., 2004; Wise, 2004, 2006; Volkow and Wise, 2005; Baler and Volkow, 2006). These cells also play a key role in the development of addiction (Wise, 2004, 2006; Volkow and Wise, 2005). Interestingly, fasting increased DA tone and re-feeding produces an increase of DA release into the nucleus accumbens (Wang et al., 2002). Anticipation of food and other rewards also produce DA release from the VTA into the nucleus accumbens (Blackburn et al., 1992; Richardson and Gratton, 1996). In addition to DA-related processes, serotonergic components of the raphe nucleus have been implicated in the regulation of food intake (Heisler et al., 2003; Halford et al., 2007). Indeed, there are serotonin (5-HT) projections to hypothalamic and limbic centres involved in the regulation of food intake (Heym and Gladfelter, 1982; Vertes et al., 1999; Brown and Molliver, 
2000; Silva et al., 2002; Jankowski and Sesack, 2004). Several lines of evidence demonstrate that 5-HT has an inhibitory effect on food intake, including clinical and experimental data on anorectic effects of 5-HT reuptake inhibitors (i.e. SSRI's) (Halford et al., 2007). The effects of 5-HT on food intake and metabolic function are mediated, at least in part, through the stimulation of POMC cells in the hypothalamic ARC, as well as through the direct effects of 5-HT onto cells of the PVN (Heisler et al., 2003).

Stress influences these midbrain regions, and chronic stress produces long lasting changes in the monoaminergic neurotransmitters produce and released by cells in these structures (Anisman et al., 2008). Nevertheless, stimulation of DA and 5-HT seem to be counter regulatory responses to the stress and appear to promote behaviours associated with coping including the consumption of palatable comfort foods to reduce stress (Dallman et al., 2006).

\subsubsection{Stress \& Metabolic Hormones Interact to Regulate Energy Balance}

In response to stress, there are several peptides and hormones released both centrally and peripherally that are capable of acting on many hypothalamic centres, which will inevitably dictate the feeding behaviours generated in response to a stressor. There are countless transmitters, peptides and hormones released directly or indirectly, in response to stressful stimuli (See Anisman and Matheson (2005); Black (2006) for reviews). Here, we will review a subset of these messengers and discuss briefly how they relate to feeding responses following stress.

\section{Glucocorticoids}

Traditionally, the development of stress-induced metabolic disturbances has been attributed to the hypersecretion of glucocorticoids (cortisol in humans and corticosterone in rodents) as a consequence of HPA axis hyperactivity (Bartolomucci et al., 2009; Black, 2006; Dallman et al., 2006; Dallman and Warne, 2007; Rebuffé-Scrive et al., 1992). Both human and rodent obesity, with an emphasis on visceral obesity, have been associated with increased HPA axis activity and subsequent rises in glucocorticoid concentrations that can affect both the brain and peripheral tissues (Weaver et al., 1993). Excess production of glucocorticoids, as seen in patients with Cushing's disease for example, leads to increases in central adiposity and additional metabolic complications (Björntorp and Rosmond, 1999, 2000). The mechanisms by which glucocorticoids promote central adiposity remain controversial and largely uncharacterized. Like other steroid hormones, the lipophilic glucocorticoids gain access to the brain via the blood brain barrier (Castonguay, 1991) and influence the expression of hypothalamic peptides involved in the regulation of food intake and energy homeostasis (Cavagnini et al., 2000; Savontaus et al., 2002). For example, glucocorticoids have the ability to suppress the production and subsequent release of anorectic $\mathrm{CRH}$, which may play a role in their ability to increase caloric and dietary fat intake 
(Raber, 1998; Rosmond et al., 1998). Another mechanism by which glucocorticoids promote adiposity could be through their ability to increase the amount of fatty acids available in circulation by increasing lipoprotein lipase (LPL) activity, while simultaneously escalating de novo lipid production in hepatocytes (Fried et al., 1998; Wang et al., 2004). Furthermore, it has been suggested that glucocorticoids have acute antilipolytic effects on adipocytes, which could help explain their adipogenic effects following stress, however these results remain controversial (Ottosson et al., 2000; Xu et al., 2009; Campbell et al., 2011). Expression of glucocorticoid receptors (GR) has been shown in many brain regions heavily implicated in energy homeostasis such as the ARC, LH and PVN (Morimoto et al., 1996). Given that glucocorticoids are capable of regulating behaviours that control energy input and energy expenditure, they are regarded as a key factor in the link between stress and obesity. To strengthen this association, it has been shown that bilateral adrenalectomy in rats decreases glucocorticoid concentrations as well as food intake (Germano et al., 2007), and glucocorticoid replacement in adrenalectomized animals restores food intake (Green et al., 1992; Jacobson, 1999). However, the association between glucocorticoid concentration and obesity may not be as straightforward as it was once thought to be. Glucocorticoids have been shown to stimulate expression, inhibit expression and interact with, other metabolically active hormones (e.g., insulin, leptin, CRF, and others), in turn providing a much more complex control over feeding behaviours (Brindley and Rolland, 1989; Cavagnini et al., 2000; la Fleur, 2006; Jahng et al., 2008). As previously mentioned, all stressors possess the ability to elicit glucocorticoid secretion. No two stressors, however, elicit the same response from the HPA axis or the same feeding response. The degree to which HPA axis becomes activated, and thus the amount of glucocorticoids produced in response to the stressor, depends in large part on the length and severity of the stressor at hand (Martí et al., 1994; Harris et al., 1998; Vallès et al., 2000).

\section{Ghrelin}

Despite glucocorticoids being considered the primary hormonal culprit behind the development of stress-induced metabolic disturbances, other metabolically active hormones may also play a role in this process. One of these potential hormones is the gut derived hormone ghrelin. Ghrelin is a 28 amino acid hormone peptide produced in the $\mathrm{X} / \mathrm{A}$-like cells of the gastric oxyntic mucosa lining the stomach (Dornonville de la Cour et al., 2001). The X/A-like cells constitute 20-30\% of all oxyntic endocrine cells and, in addition to ghrelin, express a number of other metabolically active hormones such as obestatin, nesfatin-1 and des-acyl-ghrelin (Zhang et al., 2005; Stengel et al., 2009). Although the function of obestatin remains controversial, the cumulative evidence suggests that obestatin, nesfatin-1 and des-acyl-ghrelin all have anorexic properties making ghrelin the only orexigenic peptide expressed in these cells (Kobelt et al., 2008; Shimizu et al., 2009; Inhoff et al., 2009). Intuitively, these cells are extremely sensitive to metabolic signals in the circulation and change their protein expression profile accordingly. For example, CCK and insulin suppress ghrelin produc- 
tion in the X/A-like cells, while adrenalin, noradrenalin and endocannabinoids increase ghrelin levels (Brennan et al., 2007; Saad et al., 2002; de la Cour et al., 2007; Zbucki et al., 2008).

Since its discovery by Kojima et al. (1999), ghrelin has been chiefly ascribed as a major player in the maintenance of both short term and long term energy homeostasis, as well as a key regulator of physiological processes such as glucose metabolism (Broglio et al., 2003; Sun et al., 2007a), insulin secretion (Broglio et al., 2003; McLaughlin et al., 2004), gastric emptying (Masuda et al., 2000), cell proliferation (Andreis et al., 2003), memory (Carlini et al., 2002, 2004), anxiety (Asakawa et al., 2001a; Carlini et al., 2002; Lutter and Sakata, 2008) and reward (Abizaid and Liu, 2006; King et al., 2011). Acutely, ghrelin has the unique ability to promote food intake (Nakazato et al., 2001; Currie et al., 2005; Date et al., 2006) an effect that is mediated by the stimulation of distinct hypothalamic, brain stem, and midbrain nuclei (Cowley et al., 2003; Seoane et al., 2003; Abizaid and Liu, 2006). While there are countless neuropeptides that carry out anorexic functions, to date, ghrelin is the only known gut-brain peptide that encourages food intake. In addition, ghrelin contributes to the control of long-term energy homeostasis by regulating body weight and adiposity presumably by reducing lipid oxidation (Tschöp et al., 2000; Choi et al., 2003; Theander-Carrillo et al., 2006). Tschöp et al. (2000) demonstrated very elegantly that ghrelin increases respiratory quotient, thus promoting the utilization of carbohydrates as a fuel source while sparing fat stores, without effecting energy expenditure.

The ability of ghrelin to affect physiological processes relies on its affinity to the growth hormone secretagogue receptor-1a (GHSR-1a), the only known ghrelin receptor. GHSR-1a is a G-coupled protein receptor with seven transmembrane spanning $\alpha$-helices (Camiña, 2006). The molecular architecture of this receptor allows ghrelin, and other non-related growth hormone secretagogues, to bind to the transmembrane core in order to convey information across the membrane (Feighner et al., 1998). The characteristics of the binding pocket of GHSR-1a are such that the first five residues of the ghrelin sequence (GlySer-Ser-Phe-Leu) compliment and interact with specific regions of the active site (Bednarek et al., 2000; Bondensgaard et al., 2004). The responsiveness of GHSR-1a to ghrelin, and other ligands, is dictated by transcriptional regulation of its expression, as well as desensitization in the form of receptor internalization (Camiña et al., 2004; Pitcher et al., 1998). Not until recently, it was discovered that GHSR-1a remains constitutively active at roughly $50 \%$ efficacy in the absence of its ligand (Holst et al., 2003; Holst and Schwartz, 2004). The functional reasoning for constitutive activity of GHSR-1a is currently unknown. It is tempting to speculate that the constitutive activity of GHSR-1a provides a mechanism for ghrelin-signalling induced feeding at times when ghrelin levels are low (i.e. between meals). As such, anti-obesity agents have been tailored to act as inverse agaonists to GHSR-1a and therefore reduce the constitutive activity of this receptor (Holst et al., 2003). Other possible explanations for the constitutive activity of GHSR-1a are to ensure a constant control over metabolic processes such as basal metabolic rate, growth hormone secretion and lipid ox- 
idation (Howard et al., 1996; Holst et al., 2003). Holst et al. (2003) suggest the possibility of an endogenous inverse agonist, capable of regulating the constitutive activity of GHSR-1a similar to the long lasting effects of AgRP on the constitutive activity of $\mathrm{MC}_{3} / \mathrm{MC}_{4}$ receptors. Intuitively, it makes sense that an organism is capable of fine tuning the activity of receptors via multiple mechanisms.

Interestingly, activation of GHSR-1a requires post-translational modification of the mature ghrelin protein (Kojima et al., 1999), another characteristic unique to ghrelin amongst all other metabolically active hormones. Specifically, ghrelin must be acylated on the hydroxyl group of the third serine (Ser3) residue with an n-octanoic acid, or other medium-chain fatty acids (MCFA) containing 6-12 carbons, in order to bind to and activate GHSR-1a (Kojima et al., 1999). Modification with an n-octanoyl group plays an important role in the binding of ghrelin to GHSR-1a by inducing a conformational flexibility that accommodates the geometric specificity of the receptors binding pocket (Bednarek et al., 2000). It has been shown experimentally that peptides with longer octanoyl side chains are capable of activating GHSR-1a, but as the length of the side chain decreases so too does the efficiency of binding to the receptor (Bednarek et al., 2000).

The enzyme responsible for the acylation of the mature ghrelin protein is called ghrelin O-acyltransferase (GOAT) (Gutierrez et al., 2008; Yang et al., 2008a) which belongs to a superfamily of enzymes referred to as membranebound O-acyltransferases (MBOAT) (Hofmann, 2000). GOAT is extremely conserved across all vertebrate species, and is therefore considered a primitive regulator of energy homeostasis (Gutierrez et al., 2008). GOAT expression has been demonstrated in many rat, mouse and human tissues, including an exclusive distribution in ghrelin producing X/A-like cells of the gastric oxyntic mucosa lining the stomach (Sakata et al., 2009; Stengel et al., 2010; Lim et al., 2011), and has been shown to exist in circulation of both rodents and humans (Stengel et al., 2010; Lim et al., 2011; Stengel et al., 2011). The activity of GOAT is thought to rely on the availability of dietary lipids, however contradicting results have recently emerged with respect to peripheral GOAT expression as a function of nutritional status. According to some, GOAT expression is up-regulated following an acute fast and circulating plasma GOAT levels are dependent on BMI, where low-BMI anorexic patients show low levels of circulating GOAT and high-BMI obese patients show high levels (Stengel et al., 2010; Goebel-Stengel et al., 2013). Despite the paradoxically high levels of ghrelin in anorexic patients, Goebel-Stengel et al. (2013) suggest that GOAT expression is positively correlated with both body weight and BMI. In contrast, others have found that GOAT expression is significantly decreased following an acute fast or long-term dietary restriction (Kirchner et al., 2009; Reimer et al., 2010). Regardless of these contradicting results, the connection drawn between GOAT expression and nutritional status has led to the hypothesis that GOAT acts as a sensor of nutrients, particularly lipids (Kirchner et al., 2009). To date, GOAT is the only known enzyme capable of acylating and therefore activating ghrelin, as evidenced by the complete absence of acylated ghrelin in GOAT null mice (Gutierrez et al., 2008). GOAT is therefore heavily involved in the regu- 
lation of energy homeostasis, given its monopolizing role in the production of acylated ghrelin.

GHSR-1a is widely expressed in both rodents and humans, with the highest expression found in the hypothalamus and the pituitary gland, both areas heavily invested in energy homeostasis and growth hormone secretion (Howard et al., 1996). In the mouse pituitary, GHSR-1a expression appears to peak early in development where the highest levels of expression can be observed at 1-2 months of age (Sun et al., 2007b). Thereafter, GHSR-1a expression declines by the age of 6 months and remains relatively constant throughout adulthood (Sun et al., 2007b). Importantly, GHSR-1a expression is confined to the anterior pituitary gland and is co-localized with growth hormone releasing somatotrophic cells (Reichenbach et al., 2012). In addition to murine GHSR-1a expression on GH producing pituitary cells, others have demonstrated similar patterns of GHSR1a expression in human pituitary tissues (Kim et al., 2001). Furthermore, in adult mice, there is a modest expression of GHSR-1a on ACTH producing corticotrophs (Reichenbach et al., 2012). This finding provides some direct evidence for a link between ghrelin secretion and HPA axis activity, via GHSR-1a-positive ACTH producing secretory cells in the anterior pituitary. Within the hypothalamus, GHSR-1a expression is most concentrated to the arcuate nucleus (Howard et al., 1996), a region important in the regulation of food intake, metabolism and energy homeostasis (Kirchner et al., 2009; Tschöp et al., 2000). Specifically, GHSR-1a resides primarily on a sub-set of first-order arcuate neurons that co-express the orexigenic transcripts NPY and AgRP (Lucidi et al., 2005; Willesen et al., 1999; Zigman and Jones, 2006). As previously discussed, NPY and AgRP are two potent orexigenic peptides found predominantly in the ARC and whose expression and secretions are mediated through impairments in energy balance such as depletion of body fat stores and/or reduced insulin/leptin signalling in the brain (Kalra et al., 1991; Schwartz et al., 2000; White and Kershaw, 1990; Wilding et al., 1993). It has been shown that ghrelin is capable of depolarizing NPY/AgRP neurons (Cowley et al., 2003) and that antagonists for both NPY and AgRP abolish ghrelin induced feeding (Nakazato et al., 2001). However, the ability of ghrelin to depolarize the majority of arcuate neurons is regulated by the overlapping sub-set of leptin-responsive POMC and CART neurons. Ghrelin exerts potent orexigenic actions by simultaneously activating the NPY/AgRP, and inhibiting the POMC/CART neurons in the arcuate nucleus (Currie et al., 2005; Konturek et al., 2004; Lucidi et al., 2005; Nakazato et al., 2001; Riediger et al., 2003). Once activated by ghrelin, the NPY/AgRP neurons release their orexigenic transcripts at the aforementioned downstream target sites to affect food intake and body weight via modulation of intracellular signalling cascades (Currie et al., 2005; Nakazato et al., 2001). However, the way in which ghrelin activates these hypothalamic neurons is not entirely clear. Once ghrelin has been biologically activated by GOAT and secreted by the stomach, it is thought to stimulate hypothalamic centres (e.g. ARC) by accessing the brain via the circulation, as well as suppressing the activation of vagal afferents. Indeed the ARC contains a dense expression of ghrelin receptors and is located in an area permeable to the blood brain barrier (Ciofi, 2011; 
Morita and Miyata, 2012, 2013). Furthermore, the vagus nerve also contains a dense expression of ghrelin receptors (Asakawa et al., 2001a; Date et al., 2002), suggesting that both mechanisms are viable modes of communication between ghrelin and the brain. The afferent projections from the vagus nerve to the NTS represent the strongest, and most notable, neuroanatomical mode of communication between the digestive system and the brain. Anorectic signals such as CCK are known to stimulate these vagal afferents while orexigenic signals such as ghrelin inhibit vagal afferents (Date et al., 2002). While it is still believed that ghrelin is capable of accessing the brain via the bloodstream, several studies demonstrate that peripheral ghrelin injections into vagotomized animals did not induce cFos activation in NPY/AgRP neurons (Asakawa et al., 2001b; Date et al., 2002). These data suggest the orexigenic effects of ghrelin are relayed by the vagus nerve to the hypothalamus, and are therefore dependent on intact vagal nerve communications. However, vagotomized animals still responded in a predictable manner to central administration of ghrelin (Date et al., 2002), indicating a role for central ghrelin in the control of food intake despite the dependency on vagal nerve communication for peripheral-ghrelin induced feeding. It remains unclear exactly how the gut-peptide ghrelin communicates with the brain, and the respective roles of vagal-mediated communication vs. direct hypothalamic activation.

The precise intracellular mechanisms downstream of GHSR-1a activation that lead to the eventual synthesis and secretion of orexigenic peptides onto second order hypothalamic neurons remains to be elucidated. Understanding how hormonal signals such as ghrelin regulate intracellular signalling cascades to control whole body energy homeostasis may lead to therapeutic targets to treat metabolic disorders such as obesity. To date, several intracellular signalling cascades are thought to mediate the orexigenic properties of GHSR-1a activation (Andrews et al., 2008; Sangiao-Alvarellos et al., 2010; Velásquez et al., 2011; Verhulst et al., 2012). In particular, the adenosine mono-phosphate protein kinase (AMPK) pathway has gathered the majority of attention for its importance in ghrelin signalling (See Figure 1.1). At the level of the hypothalamus, AMPK coordinates mitochondrial fatty acid oxidation by regulating a series of enzyme-catalyzed reactions, ultimately increasing carnitine-palmitoyltransferase- $1 \alpha(\mathrm{CPT} 1 \alpha)$ activity and lipid $\beta$-oxidation (Ha et al., 1994; Munday et al., 1988). When long-chain fatty acids (LCFA) are taken up into neurons, they are immediately converted into LCFA-CoA molecules by the enzyme fatty acid synthase (FAS). Cytoplasmic LCFA-CoA molecules serve as a fuel gauge to neurons in the hypothalamus, whereby a build-up of LCFA-CoA molecules is indicative of an energy surplus, and a reduction indicates a state of energy depletion (Lam et al., 2005; Obici et al., 2002, 2003). Once inside the cell, CPT1 $\alpha$ is responsible for shuttling the LCFA-CoA molecules into the mitochondria where they undergo $\beta$-oxidation and generate cellular ATP (McGarry et al., 1978). The rate at which LCFA-CoA molecules are sent to the mitochondria to undergo $\beta$-oxidation is entirely dependently on the AMPK activity upstream of CPT1 $\alpha$. Following its phosphorylation, phosphorylated AMPK (pAMPK) phosphorylates acetyl-CoA carboxylase (ACC), at the Ser79 residue, rendering 
the enzyme inactive (Ha et al., 1994; Munday et al., 1988). Normally, ACC catalyzes the conversion of acetyl-CoA to malonyl-CoA which allosterically inhibits CPT1 $\alpha$ activity (McGarry et al., 1978). Therefore, upon inactivation of ACC by pAMPK phosphorylation, malonyl-CoA production is stopped which removes inhibition of CPT1 $\alpha$. Finally, upon removal of CPT1 $\alpha$ inhibition, cytoplasmic LCFA-CoA molecules are shuttled into the mitochondria, depleting cytoplasmic LCFA-CoA levels and signalling a state of energy depletion therefore promoting food intake (Andersson et al., 2004; Gao and Horvath, 2007; Hardie and Carling, 1997; López et al., 2008; Verhulst and Depoortere, 2012).

Enzymes involved in the AMPK-ACC-fatty acid oxidation pathway are densely expressed in the hypothalamic NPY/AgRP and POMC/CART neurons that express GHSR-1a (López et al., 2008). Intraperitoneal administration of ghrelin has been shown to induce AMPK phosphorylation, decrease FAS mRNA expression and increase ACC phosphorylation (pACC) in the hypothalamus (Andersson et al., 2004; Kola et al., 2005; López et al., 2008). The effects of ghrelin on FAS expression and ACC activity ultimately reduce hypothalamic malonyl-CoA production and cytoplasmic LCFA-CoA levels (López et al., 2008), signalling an energy-depleted state to the organism and therefore promoting food intake. In contrast, the effects of anorectic peptides such as insulin and leptin have been shown to decrease AMPK activity in the hypothalamus, which in turn leads to increases in cytoplasmic LCFA-CoA molecules signalling an energy surplus and thus reducing food intake (Andersson et al., 2004). There also exists other signalling cascades downstream of GHSR-1a that may too play a role in ghrelins biological activities. For example, it has been shown that intracellular calcium $\left(\left[\mathrm{Ca}^{2+}\right]_{i}\right)$ is dose-dependently elevated in $80 \%$ of NPY neurons in response to ghrelin administration, and that $\mathrm{N}$-type $\mathrm{Ca}^{2+}$ channels are responsible for the $\mathrm{Ca}^{2+}$ influx (Kohno et al., 2003, 2007). Interestingly, this effect is completely abolished with co-administration of the adenylate cyclase (AC) inhibitor SQ22536, the PKA inhibitor H89, and/or the phospholipase C (PLC) inhibitor U73122 but not the PKC inhibitor H85, highlighting the role of the adenylate cyclase PKA and PLC signalling cascades in ghrelin induced activation of arcuate NPY neurons (Kohno et al., 2003, 2007). It remains to be fully understood, however, the relative contribution of each independent signalling cascade on the various physiological functions of ghrelin (i.e increasing food intake vs. increasing carbohydrate utilization vs. GH secretion). While it is well established that AMPK phosphorylation downstream of ghrelin induced GHSR-1a activation in the hypothalamus is responsible for generating the orexigenic effects of ghrelin, it has been suggested that both $\mathrm{AC} / \mathrm{PKA}$ and PLC/PKC activation are responsible for stimulating GH secretion (Malagón et al., 2003). Supporting this claim is evidence demonstrating that the $\mathrm{PKC}$ inhibitor phloretin is capable of abolishing ghrelin induced GH secretion, without affecting basal GH secretion (Malagón et al., 2003). Similarly, the PKA inhibitor H89 completely abolishes ghrelin induced GH secretion from pituitary somatotrophs (Malagón et al., 2003).

It is well established that ghrelin promotes the utilization of carbohydrates as an energy substrate while sparing the utilization of body fat, ultimately tipping 
the energy homeostatic scales in favour of adiposity (Tschöp et al., 2000). It is believed ghrelin exerts its orexigenic and adipogenic effects through its influence on the AMPK signalling pathway. Interestingly, plasma ghrelin levels have been shown to rise in parallel with glucocorticoids in response to both acute and chronic stress, and ghrelin levels remain elevated for an extended period of time after the cessation of the stressor (Asakawa et al., 2001a; Kristenssson et al., 2006; Ochi et al., 2008). For example, acute water avoidance stress in rats has been shown to elicit an $85 \%$ increase in circulating ghrelin 1 hour after cessation of the stressor (Kristenssson et al., 2006). Similarly, chronic social defeat stress has been shown to increase circulating acylated ghrelin levels (Lutter and Sakata, 2008; Chuang and Perello, 2011), a change that persists up to 30 days after the stress paradigm has been terminated (Lutter and Sakata, 2008). The mechanism responsible for stress-induced ghrelin secretion is not fully understood, however it is thought to be mediated, in part, by sympathetic tone. It has been shown that both chemical and electrical stimulation of the sympathetic nervous system, mimicking activation caused by an acute stressor, resulted in a significant increase in circulating plasma ghrelin (Mundinger et al., 2006). Interestingly, this effect remained uninterrupted in streptozotocin-induced diabetic rats with chronically high insulin levels suggesting that neural sympathetic nervous system activation, and not neurohormonal components, was responsible for eliciting ghrelin secretion (Mundinger et al., 2006). Injections of epinephrine, on the other hand, have produced somewhat divergent results. Some show that an injection of enough epinephrine to mimic a severe stressor was not sufficient enough to elicit any change in circulating ghrelin levels (Mundinger et al., 2006). Others have shown that epinephrine injections decreased fasting ghrelin levels (Joibari and Khazali, 2013). While these data are by no means conclusive, or in agreement with each other, together they provide a direct line of evidence linking sympathetic nervous system activation and stress-induced ghrelin secretion. In support of this claim, others have used retrograde tracers to demonstrate the existence of selective noradrenergic innervation of central vagal projections with the fundus of the rat stomach, the precise area where acylated ghrelin is typically harboured (Pearson et al., 2007; Ariyasu, 2001). Most neurons and cell bodies that were found to project to the fundus originated in the hind brains area postrema (AP) (Pearson et al., 2007). Furthermore, it has been shown that ghrelin-secreting cells express high levels of mRNA encoding $\beta_{(1)}$-adrenergic receptors, and direct application of norepinephrine and/or epinephrine onto these cells results in ghrelin secretion (Zhao et al., 2010), an effect that is abolished in the presence of a $\beta_{(1)}$-adrenergic receptor antagonist (Zhao et al., 2010).

Administration of ghrelin, both centrally and peripherally, has been shown to indirectly stimulate hypothalamic CRF neurons, leading to HPA axis activation (Cabral et al., 2012). Ghrelin deficient mice show a reduction in the number of CRF expressing cells in the PVN and demonstrate a hyporesponsive HPA axis in response to acute stress (Spencer et al., 2012). Interruption of ghrelin signalling, both genetically and pharmacologically, during times of chronic stress can improve the metabolic outcome of these animals suggesting that ghrelin does in fact contribute to the development of stress-induced metabolic disturbances 
(Lutter and Sakata, 2008; Chuang and Perello, 2011). Although it is generally accepted that ghrelin stimulates food intake in response to a stressor, it remains unclear what type of food is encouraged following a stress-induced secretion of ghrelin. Chuang and Perello (2011) have very nicely demonstrated that stressinduced ghrelin secretion mediates increases in high-fat diet consumption, as well as contributes to a stronger conditioned place preference toward a high-fat diet following a chronic stressor. Thus, mice may engage in reward-based eating of comfort foods and develop a conditioned place preference to such foods after being stressed (Chuang and Perello, 2011), however it is unknown how chronic social stress dictates food intake during the stress paradigms.

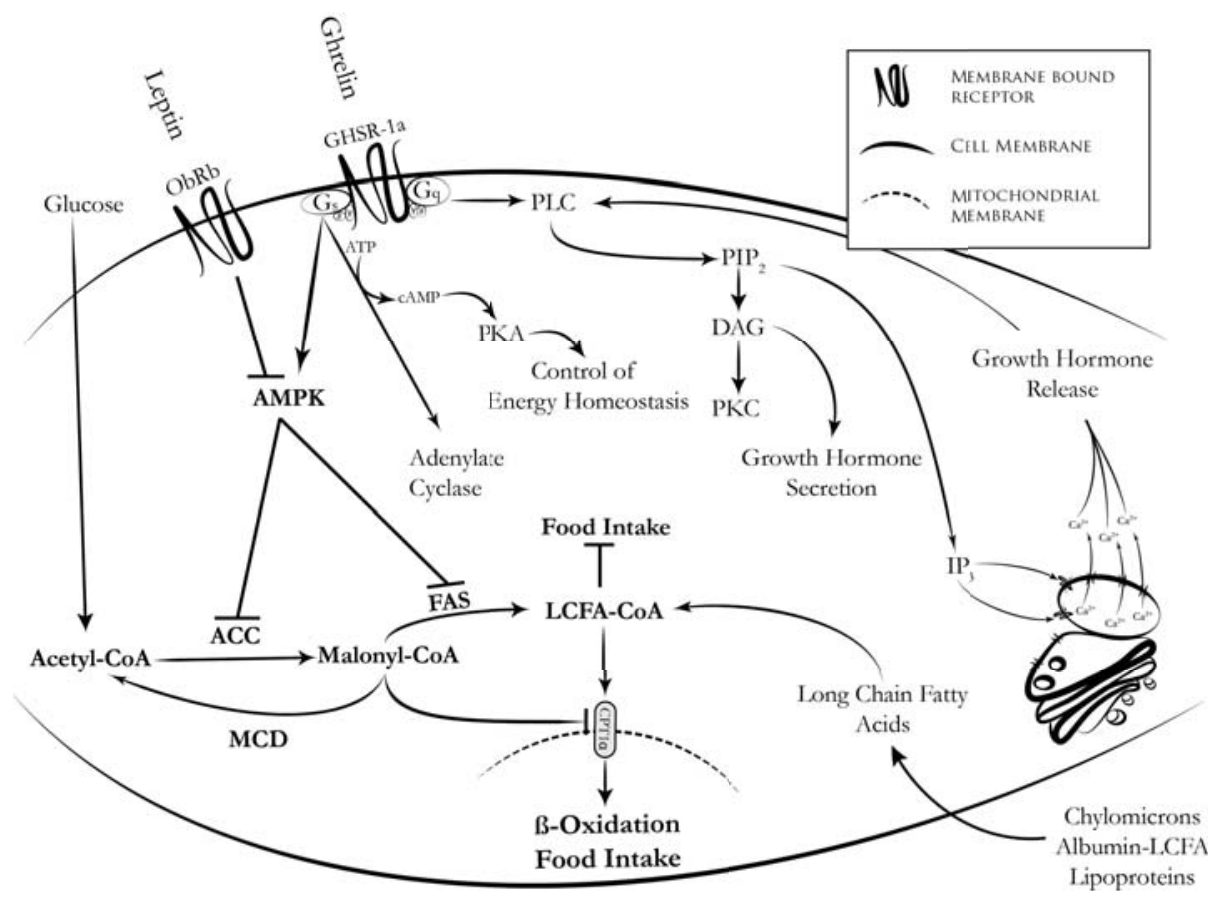

Figure 1.1: Hormonal influence of hypothalamic AMPK-mediated mitochondrial fatty acid $\beta$-oxidation, phospholipase $\mathrm{C}$ and protein kinase $\mathrm{A}$ signalling in response to ghrelin and/or leptin receptor activation 


\section{Leptin}

Leptin is another peripherally derived hormone, expressed in accordance to the size and amount of adipocytes, that is known to exert some control of feeding behaviours and energy homeostasis. Once secreted from peripheral adipocytes, leptin acts on hypothalamic centres (e.g. ARC) to reduce food intake and increase energy expenditure, thus encouraging a negative energy balance (Friedman, 2002). Leptin is also capable of downregulating the expression of orexigenic hormones such as ghrelin (Kojima and Kangawa, 2005). It has been suggested that leptin is also involved in the metabolic consequences of stress. Circulating glucocorticoids, for example, increase leptin mRNA expression and its secretion from adipocytes (Hardie et al., 1996; Russell et al., 1998; Williams et al., 2000). This pattern of direct glucocorticoid mediated stimulation of leptin secretion has been shown in both humans and rodents (Miell et al., 1996; Mostyn et al., 2001). Once secreted, leptin directly stimulates anorectic POMC/CART neurons in the ARC and mediates the ability of other metabolically active hormones like ghrelin, for example, to depolarize these neurons (Elias et al., 1998; Cowley et al., 2001). Furthermore, it has been shown that leptin shares an overlapping physiological and intracellular signalling cascade with hormones such as insulin (Niswender and Schwartz, 2003) thus exerting control of feeding circuits from multiple standpoints. There is, however, contradicting data regarding interactions with leptin and glucocorticoids thus making it difficult to interpret the effects of leptin in the context of stress-induced feeding and/or changes in body weight. One group has shown that IP administration of glucocorticoids (mimicking levels seen following an acute stressor) increases leptin concentrations and therefore results in a decrease in food intake and body weight (Zakrzewska et al., 1999a). However, the same group demonstrated an opposing role for leptin when glucocorticoids were administered centrally, wherein NPY expression was increased and so too were food intake and body weight (Zakrzewska et al., 1999b). It remains unclear how leptin contributes to stress induced metabolic disorders and more studies are required in order to dissociate the interactions between central vs. peripheral leptin and glucocorticoids in response to stressful stimuli.

\section{Others}

In addition to corticosterone, ghrelin and leptin, there are a host of hormones that are stimulated by stress and that contribute directly to the regulation of food intake and energy balance. For instance, cytokines, both inflammatory and anti-inflammatory, are secreted following acute stressors (Black, 2006; Anisman et al., 2008). Chronic stressors, however, result in high levels of cytokines like IL-6 and TNF- $\alpha$, both of which may contribute to obesity by causing peripheral and central leptin resistance (Velloso et al., 2008). Furthermore, while insulin may not be directly related to the stress response, its interactions with glucocorticoids allow it to participate in shaping an organism's phenotype in response to stress. For example, in the presence of insulin glucocorticoids shift their focus 
from mobilization of lipids to lipid accumulation (Ottosson et al., 2000). In addition, the interaction between insulin and elevated levels of glucocorticoids has been linked to increased obesity following overconsumption of comfort foods (Dallman et al., 2005). For more detailed review on the neurochemical control of food intake see Maniam and Morris (2012).

\subsection{Stress Models}

There are many disorders/diseases that are studied through the use of animal models, and obesity is no different. Over the past century, many different stress models have been designed, some in attempt to study the effects of stress on depression or anxiety, and others to directly study the effects of stress on food intake and obesity. While it is well-documented that stress is associated with changes in body weight in rodents (Barnett, 1958; Barnett et al., 1960; Martí et al., 1994) it is not always the case that animal models mimic human reality and the basis of dissimilar findings remains poorly understood. Here, we will examine several different stress models used in the context of obesity and discuss the benefits and pitfalls of each.

\subsubsection{Visible Burrow System}

This visible burrow system (VBS) was originally developed as a naturalistic social stressor in attempt to investigate agonistic behaviours in rats (Blanchard et al., 1977; Blanchard and Spencer, 1995). This model reproduces an underground burrow system, which is thought to mimic the natural habitat of a rat. Traditionally, four adult male rats, and two adult female rats, are group housed in the VBS and a social hierarchy is rapidly formed within days yielding one dominant and three submissive male animals (Tamashiro et al., 2006). This provides a very unique scenario for scientists interested in studying aggressive behaviours in rodents in a "naturalistic" setting wherein there is little requirement for intervention by the experimenter. Since its inception, others who are more interested in studying the neuroendocrine systems involved with stress, feeding and energy homeostasis have adopted the VBS. Inside the VBS, submissive animals show increased basal CORT (Blanchard et al., 1993; Blanchard and Spencer, 1995; McKittrick et al., 1995, 2000), increases in testosterone (Blanchard and Spencer, 1995; Hardy et al., 2002; Tamashiro et al., 2004) and reliably lose $\approx 10-15 \%$ of their body weight (Blanchard and Spencer, 1995; Hardy et al., 2002; Tamashiro et al., 2004). This reduction in body weight has been carefully attributed to decreases in food intake and changes in metabolic rate, while other factors such as basal locomotor activity have been eliminated as contributing factors (Tamashiro et al., 2004, 2006). The molecular underpinnings of such a robust and reliable reduction of food intake are not entirely clear. The VBS has been shown to elicit no change in the expression of orexigenic peptides or hormones, but rather elicits a reduction of leptin and insulin in submissive animals. More experiments are required for a detailed description of changes in 
hypothalamic feedings circuits that underlie the metabolic outcome of this stress paradigm. As a result of decreased food intake and changes in metabolic rate, submissive animals in the VBS show reductions of adiposity and a decline in lean body mass (Tamashiro et al., 2004). Interestingly, if the animals are removed from the VBS and allowed to recover, in attempt to correct their metabolic phenotype, they show a restoration of most physiological and endocrine measures, but will not regain body weight to levels equivalent to dominant or non-stressed control animals (Tamashiro et al., 2006). During the recovery period, animals immediately become hyperphagic and it has been shown that the gradual accumulation of body weight during a 3 week recovery from the VBS is the result of increased visceral adipose tissue (Tamashiro et al., 2006). Interestingly, the animals display persistent anhedonia and will consume less sucrose during the recovery period, compared to controls (Tamashiro and Hegeman, 2007).

In this regard, it would appear that the VBS is not an ideal stress paradigm for the study of obesity. While there are certainly some well-documented increases in adipose tissue observed in submissive animals, this is a relative measurement to where the animal was following participation in the VBS. In absolute terms, these animals show an overall reduction of food intake, body weight and visceral adipose tissue and therefore do not mimic human cases of stressinduced obesity. Perhaps these findings are of no surprise given the endocrine profile of these animals. Increased testosterone, for example, as seen in submissive animals has been shown to predict reduced adipose tissue (Krotkiewski et al., 1980). Interestingly, in the absence of females inside the VBS, submissive animals do not show any changes in body weight (Tamashiro and Hegeman, 2007). It is possible that competition for resources (food, water, enrichment, females, etc.) results in submissive animals lacking the energetic resources required to develop an obese-like phenotype.

\subsubsection{Resident Intruder Paradigm}

The resident intruder paradigm, also known as social defeat stress has been used as a method for studying the effects of social dominance and subordination in animals (Ginsburg and Allee, 1942; Miczek, 1979; Miczek et al., 1982). Social hierarchies result in the expression of dominant and submissive behavioural and neuroendocrine patterns that can be used to study the effects of chronic stressors. When compared to other types of stress paradigms such as foot shock or restraint stress, the resident intruder paradigm appears to elicit the most robust physiological response, as measured by changes in hormonal and cardiovascular function (Koolhaas et al., 1997; Sgoifo et al., 1999; Keeney and Jessop, 2006). The territorial nature of rodents allows experimenters to manipulate social hierarchies in attempt to study the physiological consequences of chronic social stressors. When an animal is single housed for $\approx 1$ week, there is a rapid development of territorial behaviour within the home cage and this animal is said to be the resident (Bartolomucci et al., 2001). Traditionally, in the resident intruder paradigm, the experimenter presents a novel, non-littermate rodent to the resident inside the home cage. The novel animal is thus considered an intruder and 
will have to fight for his (typically performed in males only) position in the social hierarchy within that cage. It has been shown that housing animals with litter mates, while permitting the formation of social hierarchy, has no influence on CORT production or anxiety-like behaviours in submissive animals and is thus considered non-stressful (Bartolomucci et al., 2001). In contrast, when housed with a novel, non-sibling rodent, submissive animals show signs of stress such as decreased immune responses (Bartolomucci et al., 2001), increased food intake, increased body weight and fat mass (Haller et al., 1999; Bhatnagar and Vining, 2003; Foster and Solomon, 2006). It is therefore necessary to use non-familiar animals when attempting to elicit metabolic changes as a consequence of social defeat stress.

Normally, this paradigm consists of two male rodents, one resident and one intruder. There are several variants of the resident intruder paradigm, all of which reflect differences in predictability, habituation, intensity and ultimately the consequent phenotype. Typically, the intruder is presented in the home cage of the resident and they are allowed to interact until one animal subdues the other. Following, interaction, animals are separated by a central divider hemisecting the home cage of the resident (Bartolomucci et al., 2001). This divider permits the exchange of sensory information but forbids any physical contact between the animals. By lifting this divider and allowing the two animals to interact, the experiment presents an opportunity for the animals to establish a social hierarchy, creating four possible groups: resident-dominant, residentsubordinate, intruder-dominant and intruder-subordinate (Bartolomucci et al., 2001). The physiological outcome in animals exposed to the resident intruder paradigm seems highly dependent on the nature of the social hierarchy (Bartolomucci et al., 2001; Bartolomucci and Pederzani, 2004; Bartolomucci and Palanza, 2005; Solomon et al., 2007). Reversal of social status in mice, for example, seen when dominant resident becomes submissive to the intruder has been associated with immunosuppression (Devoino et al., 2003) and dramatic increases in body weight (Bartolomucci and Pederzani, 2004), while residents who did not lose their dominant status showed no changes in immune function (Devoino et al., 2003) and in some cases show reductions in body weight (Bartolomucci and Pederzani, 2004). The emergence of aggressive and dominant behaviour in non-defeated intruders is considered an immunostimulant (Devoino et al., 2003) and these animals show resistance to the development of obesity, as well as predictable allocation of adipose depots (Solomon et al., 2007). In the context of chronic social defeat stress, some laboratories choose novel residents each day of defeat (Bhatnagar and Vining, 2003; Devoino et al., 2003; Foster and Solomon, 2006; Keeney and Jessop, 2006; Krishnan et al., 2007; Lutter and Sakata, 2008; Lutter et al., 2008; Chuang and Perello, 2011). By employing novel residents, the experimental subjected are faced with a new social hierarchy within each unique episode of defeat. In contrast, other laboratories reuse the resident, thus maintaining an exclusive social hierarchy for the duration of the experiment (Bartolomucci et al., 2001; Bartolomucci and Pederzani, 2004; Bartolomucci, 2010; Moles et al., 2006; Solomon et al., 2007). The former prevents habituation through predictability and the latter leads to eventual habituation 
and is thus considered a milder form of chronic social defeat.

Interestingly, the metabolic phenotypes generated in concert with positions in social hierarchies can be substantiated by the hormonal profile of the animals. Submissive animals in the resident intruder paradigm, for example, show elevations in CORT and blood glucose, but also increases in circulating orexigenic/adipogenic plasma ghrelin (Lutter and Sakata, 2008). Furthermore, hormonal changes that influence metabolic phenotype may vary across time throughout a chronic social defeat stress paradigms. Some physiological changes, for example, are seen immediately following an episode of defeat (Bhatnagar and Vining, 2003; Bartolomucci and Pederzani, 2004; Bartolomucci and Palanza, 2005; Foster and Solomon, 2006; Keeney and Jessop, 2006; Lutter and Sakata, 2008) whereas others do not emerge until the subject has been removed from the stress paradigm and allowed to recover (Keeney and Jessop, 2006; Moles et al., 2006; Solomon et al., 2007; Lutter and Sakata, 2008; Chuang and Perello, 2011).

There are several advantages to using the resident intruder paradigm in attempt to study the physiological consequences of chronic stress. One distinct advantage is there has been a clear dissociation between food intake and body weight changes, where stressed animals will show distinct changes in food intake, thus suggesting that stress-induced changes in body weight and body composition are not solely attributed to increases or decreases in food intake (Haller et al., 1999; Bhatnagar and Vining, 2003; Bartolomucci and Pederzani, 2004; Moles et al., 2006; Solomon et al., 2007). Some groups have taken this one step further and dissociated the social stress aspect from the physical stress aspect of an episode of defeat and shown that social stress alone can produce changes in caloric intake (Moles et al., 2006). Interestingly, these changes in metabolism appear to extend beyond the last social interaction between resident and intruder. Subordinate animals in the resident intruder paradigm, for example, continue to show increases in body mass even weeks after their last social defeat, when levels of food intake were equivalent to non-stressed controls (Solomon et al., 2007) suggesting that this stress paradigm left an imprint on the metabolism of these animals. This increase in body mass has been attributed to the ghrelin-dependent shift in carbohydrate utilization resulting in an increase in visceral adipose tissue (Bartolomucci et al., 2001; Bartolomucci and Pederzani, 2004) and an increase in overall lipid content (Moles et al., 2006; Solomon et al., 2007).

Another advantage to using the resident intruder paradigm is its apparent external validity. It has been well-documented in human cases that a higher position on a social hierarchy (as measured by socioeconomic status, for example) is associated with decreased reported stress levels (?Sujoldzić and De Lucia, 2007; Burdette and Hill, 2008), healthier diets (McEwen, 1998; Cartwright et al., 2003; Burdette and Hill, 2008), decreased body mass index (BMI) (George et al., 2005; Brunner et al., 2007; Burdette and Hill, 2008), etc. On the contrary, being lower on the socioeconomic hierarchy has been associated with increased stress and increased BMI (Hellerstedt and Jeffery, 1997; Laitinen et al., 2002; George et al., 2005; ?), thus shaping the trends in obesity such that humans are likely to 
be healthier and more fit then those beneath them on the socioeconomic ladder, regardless of their starting position. While the VBS also involved a similar type of stressor, one major difference between these two paradigms is food availability. Because a divider in the resident intruder paradigm separates the animals, they are granted unimpeded access to ad libitum calories, as is seen in human cases of chronic social stress.

Finally, while some stressors have anorectic effects, this particular stress paradigm causes submissive animals to overeat. Overall, animals placed in the resident intruder paradigm eat more foods that are high in fat relative to nonstressed controls, but subordinate animals show increases in high fat diet intake relative to their dominant counterparts (Moles et al., 2006). However, it should be noted that the consumption of high fat diets as a consequence of chronic stress varies from one study to another and may depend on other factors such as diet availability (time and location), gender and animal strain.

While acute social defeat is considered to be a potent stressor by means of HPA axis activity (ACTH, CORT, immune function, etc.) within hours of a defeat episode (Koolhaas et al., 1997; Sgoifo et al., 1999; Keeney and Jessop, 2006) chronic social defeat stress results in significant habituation wherein defeat stress elicits a bi-phasic response from the HPA axis (Keeney and Jessop, 2006). Within the first 24 hours, a dramatic increase in CORT is evident following an episode of defeat. If the paradigm is continued, the rise in CORT in response to an episode of defeat is blunted for the next 2 weeks, but will show another spike if the paradigm is continued beyond 2 weeks (Keeney and Jessop, 2006). Furthermore, there is a discrepancy in food intake, hypothalamic CRF expression and glucocorticoid secretion between acute vs. chronically defeated animals (Keeney and Jessop, 2006; Moles et al., 2006). These data are indicative of an adaptive stress response, whose ability to downplay the potency of the stressor is limited and may become detrimental in response to chronic social defeat (Keeney and Jessop, 2006).

\subsubsection{Chronic Mild Stressors}

Chronic mild stress (CMS) has been well-documented for its ability to produce depression and the wide range of accompanying physical, behavioural and neurochemical changes that accompany depression (e.g. anhedonia) (Willner et al., 1992; Willner, 1997; Stöhr et al., 2000). Generally speaking, chronic mild stress is composed of daily stressors generated randomly from a select list of possible stressors, however, CMS protocols can vary substantially from one laboratory to the next. Daily stressors could be housing the animal in a small cage or a cage with wet bedding, housing in an overcrowded cage, cage tilt, foot shock, tail pinch, changes to light/dark cycle (24 hours of light, for example), housing in a soiled cage, predator odour, loud noises and more (Willner, 1991, 1997; Muscat and Willner, 1992; Willner et al., 1992; Stöhr et al., 2000; Duncko et al., 2001; Westenbroek et al., 2003; Patterson and Ducharme, 2010).

The most notable physiological consequence of CMS is the induction of anhedonia, however, this model is also capable of producing impairments in condi- 
tioned place preference, reductions in brain stimulation reward, decreased sexual behaviour, compromised immune function and irregular sleep architecture (Willner, 1997). These consequences of CMS are reminiscent of the physiological and neurochemical/neuroendocrine changes observed in human cases of depression, and many consequences of CMS can be corrected with chronic antidepressant administration (Willner et al., 1992; Moreau et al., 1994). Because chronic, but not acute, treatment with generic antidepressants can consistently reduce the consequences of CMS, it is considered one the best animal models of depression.

However, very few studies that use CMS elicit an obese like state in their experimental animals. Instead, it has been shown that CMS elicits decreases in the consumption of highly palatable foods (Muscat and Willner, 1992) and subsequently body weight (Muscat and Willner, 1992; Westenbroek et al., 2003). It is difficult to map out the hormonal and neurochemical responses as a consequence of CMS due to the inherent variability of stress paradigms. Within any given CMS protocol exists multiple forms of stressors, each capable of eliciting a unique feeding response. Furthermore, it is difficult to draw similarities between animal models of CMS and any type of human chronic stress. As previously mentioned, any given stressor poses a distinct physiological challenge to an organism and therefore elicits a distinct response. One must consider what type of CMS can be applied to human scenario before attempting to model human obesity.

\subsubsection{Physical Stressors}

Despite the growing body of evidence suggesting that psychological stress is one of the largest sources of human stress and may in fact contribute to the obesity epidemic, most animals models aimed at investigating the role of stress on metabolic processes focus on physical, rather then psychosocial, stressors (Tamashiro et al., 2006). Physical stressors are often used in tandem with other forms of stressors in chronic stress models (e.g. UCMS) or, more frequently, as acute stressors on their own.

\section{Tail Pinch \& Foot Shock}

Tail pinch and foot shock, for example, are two physical stressors used commonly and have both been shown to have an impact on feeding behaviours in rodents. It was shown very early on that chronic tail pinch in rats is capable of eliciting the consumption of an energy rich diet (Rowland and Antelman, 1976). In this particular experiment the rats were encouraged to consume calories through the presence of a highly palatable, energy rich diet. It is unclear if tail pinch is sufficient enough to induce physiological changes capable of influencing dietary habits. Rather, it has been suggested that these feeding responses are a product of general arousal as opposed to an induction of motivated feeding behaviours (Greeno and Wing, 1994). Furthermore, it has been shown that an increase in feeding behaviour is not observed in all subjects exposed to tail pinch, and is therefore thought to produce unreliable feeding responses in rodents (Levine 
and Morley, 1981).

Foot shock, on the other hand, has been shown to increase circulating plasma glucose, insulin and leptin in rats (Farias-Silva et al., 2002; Solomon et al., 2007) suggesting that this type of stressors results in an anorectic hormonal profile. The hyperglycemia and hyperleptinemia observed in animals exposed to foot shock has been attributed to insulin sub-sensitivity in adipose tissue (Farias-Silva et al., 2002). Despite the anorectic hormonal profile in response to repeated foot shock, animals show no change in food intake but still maintain a positive energy balance and show greater cumulative weight gain relative to non-stressed controls (Solomon et al., 2007). The changes in body mass and body composition following repeated foot shocks are said to be reminiscent of the metabolic consequences of subordinate animals in the intruder resident paradigm, albeit occurred at a much faster rate (Solomon et al., 2007).

\section{Restraint \& Immobilization Stress}

Physical restraint and immobilization are other commonly used methods of acute physical stressors. Restraint stress is regarded as a form of mild stress, as indicated by physiological and neuroendocrine responses, however, it has been shown to increase expression of orexigenic peptides in the hypothalamus nonetheless (Chagra et al., 2011). Interestingly, the expression of orexigenic peptides seems to increase when restraint stress is repeated over time (Chagra et al., 2011) and it has been shown that feeding responses to restraint stress are maintained in the absence of the stressor (Tamashiro and Hegeman, 2007). Mild restraint stress has the ability to increase the number of AgRP-expression neurons, while simultaneously reducing $\alpha$-MSH receptors, in the ARC (Chagra et al., 2011). The pattern of hypothalamic activation seems to change with repeated exposure to restraint stress. Specifically, cFos activation in the PVN, ARC and LH can be observed following acute restraint, but activation is subsided with repeated exposures suggesting habituation to this stress paradigm (Chagra et al., 2011).

Exposure to an immobilization stress, on the other hand, elicits a dramatic increase in CORT secretion and is considered to be one of the most potent stressors with respect to HPA axis hyperactivity (Haque et al., 2012). Following immobilization stress, rats have been shown to decrease their food-intake and body weight (Haque et al., 2012; Wang et al., 2012), an indication of the stressors potency. Similarly, immobilization stress has been shown to increase anorectic leptin and leptin receptor (ob-R) expression in the rat hypothalamus, particularly the ARC (Wang et al., 2012). Furthermore, no changes in NPY expression are observed following immobilization stress (Wang et al., 2012), providing hormonal data to support the feeding responses seen following acute exposure. There exists conflicting data pertaining to the potential for immobi-

lization stress to habituate (Márquez et al., 2002; Dal-Zotto et al., 2004; Wang et al., 2012). 


\section{Cold Stress}

Exposure to cold (cold stress) has been used in both rodent and human models of stress. In humans, a cold stress produces a significant rise in cortisol levels at about 15 minutes following the onset of the stressor (Geliebter et al., 2012). Normally, in stress protocols that are capable of eliciting an obese like phenotype, cortisol can be correlated to subsequent caloric intake. However, the cortisol secretion observed in human patients following a cold stress do no correlate with subsequent caloric intake and these subjects show an overall decrease in the amount of calories consumed following the stressor relative to non-stressed controls (Geliebter et al., 2012). In contrast, cold stress in rodents has been shown to increase CORT secretion and CORT secretion was directly correlated to the upregulation of hypothalamic orexigenic peptides (e.g., NPY/AgRP) (Kuo et al., 2007). Furthermore, these changes were thought to elicit proliferation and differentiation of adipocytes, ultimately producing obesity and a metabolic syndrome-like condition (Kuo et al., 2007). It remains unclear if changes in NPY circuitry in response to cold stress are an attempt to insulate/thermoregulate or if they are due to marked changes in metabolism leading to an obese like state.

Given that cold stress can be consistently applied to both human and animal subjects, but produce opposing metabolic outcomes, it is not considered to be a good model for studying stress-induced obesity. Decreasing body temperature, as can be expected during a cold stress paradigm, is metabolically expensive to an organism, as autonomic processes will immediately attempt to thermoregulate. Thermoregulation is metabolically expensive and might therefore elicit a feeding response to replenish the organisms energy stores. This is itself can be considered a confound when attempting to study stress-induced obesity. Further studies are required to better define the role of orexigenic transcripts in response to cold stress. 


\begin{tabular}{|c|c|c|c|c|c|c|c|}
\hline $\begin{array}{c}\text { Stress } \\
\text { Paradigm }\end{array}$ & Duration & Species & Intensity & $\begin{array}{l}\text { Predict- } \\
\text { ability }\end{array}$ & $\begin{array}{l}\text { Physiological } \\
\text { responses }\end{array}$ & $\begin{array}{l}\text { Food Intake/Body } \\
\text { composition }\end{array}$ & Reference(s) \\
\hline $\begin{array}{l}\text { Visual burrow } \\
\text { system }\end{array}$ & $\begin{array}{c}\text { Chronic } \\
(3 \text { weeks })\end{array}$ & $\begin{array}{l}\text { Long evans } \\
\text { rats }\end{array}$ & + & +++ & $\begin{array}{l}\text { Increased CORT } 1 / / \\
\text { decreased CORT }{ }^{2} \text {, } \\
\text { reduced leptin } \\
\text { and insulin }{ }^{2}\end{array}$ & $\begin{array}{l}\text { Decreases in food } \\
\text { intake resulting in } \\
10-15 \% \text { body } \\
\text { weight reduction }\end{array}$ & $\begin{array}{l}{ }^{1} \text { Blanchard and Spencer (1995) } \\
{ }^{2} \text { Tamashiro et al. (2004) }\end{array}$ \\
\hline $\begin{array}{l}\text { Resident Intruder } \\
\text { paradigm }\end{array}$ & Intermittent & $\begin{array}{c}\text { Syrian } \\
\text { hamsters }\end{array}$ & +++ & + & $\begin{array}{c}\text { Increased } \\
\text { leptin }\end{array}$ & $\begin{array}{l}\text { Increased food } \\
\text { intake, } \\
\text { body weight } \\
\text { and adiposity }\end{array}$ & Foster and Solomon (2006) \\
\hline $\begin{array}{l}\text { Resident intruder } \\
\text { paradigm }\end{array}$ & $\begin{array}{l}{ }^{1} \text { Chronic } \\
(10 \text { days }) \\
\left({ }^{2} \text { Chronic }\right. \\
21 \text { days })\end{array}$ & $\begin{array}{l}{ }^{1} \mathrm{C} 57 \\
\text { BL6/J Mice } \\
{ }^{2} \mathrm{CD}-1 \text { Mice }\end{array}$ & $\begin{array}{c}++ \\
++\end{array}$ & $\begin{array}{c}++ \\
+++\end{array}$ & $\begin{array}{l}\text { Increases } \\
\text { in CORT } \\
\text { ghrelin }^{1}\end{array}$ & $\begin{array}{l}\text { Increased food } \\
\text { intake; } \\
\text { no change in } \\
\text { body weight }{ }^{1} ;\end{array}$ & $\begin{array}{c}{ }^{1} \text { Lutter et al. } 2008,2008 \mathrm{~b} \\
{ }^{2} \text { Bartolomucci et al. } \\
2004,2009\end{array}$ \\
\hline Chronic mild stress & Chronic & $\begin{array}{l}{ }^{1} \text { Lister } \\
\text { hooded rats }\end{array}$ & ++ & + & Increased CORT $^{1}$ & $\begin{array}{l}\text { Anhedonia and } \\
\text { depression }^{1} ; \\
\text { Decreased } \\
\text { consumption } \\
\text { of palatable diet } \\
\text { and body wight }{ }^{1}\end{array}$ & $\begin{array}{c}{ }^{\mathrm{T}} \text { Muscat and Willner, } \\
\text { (1992) }\end{array}$ \\
\hline Foot shock & Acute (1 day) & $\begin{array}{c}\text { Syrian } \\
\text { hamsters }\end{array}$ & ++ & + & $\begin{array}{l}\text { Increased leptin, } \\
\text { no change } \\
\text { in insulin }\end{array}$ & $\begin{array}{c}\text { Increased } \\
\text { body mass, } \\
\text { feed efficiency } \\
\text { and adipose tissue }\end{array}$ & Solomon et al. (2007) \\
\hline Foot shock & $\begin{array}{c}\text { Chronic } \\
\text { (3-14 days) }\end{array}$ & $\begin{array}{c}{ }^{\mathrm{I}} \mathrm{Wistar} \text { rats } \\
{ }^{2} \mathrm{C} 57 \mathrm{BL} 6 / \mathrm{J} \\
\text { Mice } \\
{ }^{2} \mathrm{BALB} / \mathrm{c} \text { mice }\end{array}$ & ++ & ++ & $\begin{array}{l}{ }^{1} \text { Increased glucose, } \\
\text { increased insulin }\end{array}$ & $\begin{array}{l}{ }^{1} \text { Increased lipolysis } \\
{ }^{2} \text { Decrease in } \\
\text { caloric } \\
\text { consumption; no } \\
\text { change in } \\
\text { body weight }\end{array}$ & $\begin{array}{l}{ }^{1} \text { Farias-Silva et al. } \\
2002 ; \\
{ }^{2} \text { Griffiths et al. (1992) }\end{array}$ \\
\hline Tail Pinch & $\begin{array}{c}\text { Chronic } \\
\left(6 \times \text { day }^{-1} \times 5\right. \\
\text { days })\end{array} \times 5$ & $\begin{array}{l}\text { Sprague- } \\
\text { Dawley } \\
\text { rats }\end{array}$ & + & +++ & $\mathrm{n} / \mathrm{a}$ & $\begin{array}{c}\text { Increased } \\
\text { caloric intake } \\
\text { and body weight }\end{array}$ & $\begin{array}{c}\text { Rowland and } \\
\text { Antelman, (1976) }\end{array}$ \\
\hline Restraint & $\begin{array}{l}{ }^{1} \text { Acute }(1 \text { day }) \\
{ }^{2} \text { Acute }(15 \mathrm{~min})\end{array}$ & $\begin{array}{l}{ }^{\mathrm{I}} \text { Sprague- } \\
\text { Dawley rats } \\
{ }^{2} \mathrm{C} 57 \text { BL6/J } \\
\text { Mice }\end{array}$ & + & + & $\begin{array}{c}\text { Increase cFOS } \\
\text { in } \mathrm{PVN}^{1,2} \\
\mathrm{LH}^{1} \text { and } \\
\mathrm{ARC}^{1} ; \\
\text { Increased CORT }\end{array}$ & $\begin{array}{l}\text { Orexigenic effects }^{1} \\
\text { Anxiogenic effects }\end{array}$ & $\begin{array}{l}{ }^{2} \text { Chagra et al. (2011) } \\
{ }^{2} \text { Spencer et al. (2012) }\end{array}$ \\
\hline Restraint & $\begin{array}{l}\text { Chronic } \\
\text { (14 days) }\end{array}$ & $\begin{array}{l}\text { Sprague- } \\
\text { Dawley } \\
\text { rats }\end{array}$ & + & ++++ & $\begin{array}{l}\text { Increased LH } \\
\text { AgRP; } \\
\text { Decreased } \\
\text { MC4-R in } \\
\text { ARC and LH }\end{array}$ & $\begin{array}{c}\text { Increased } \\
\text { feeding-related } \\
\text { behaviours and } \\
\text { distribution } \\
\text { of energy stores } \\
\end{array}$ & Chagra et al. (2011) \\
\hline Immobilization & $\begin{array}{l}\text { Acute } \\
(2 \mathrm{~h})\end{array}$ & $\begin{array}{l}\text { Albino wistar } \\
\text { rats }\end{array}$ & ++++ & + & $\begin{array}{l}\text { Dramatic increase } \\
\text { in CORT }\end{array}$ & $\begin{array}{l}\text { Decrease } \\
\text { food intake } \\
\text { and body } \\
\text { weight }\end{array}$ & Haque et al. (2012) \\
\hline Immobilization & $\begin{array}{c}{ }^{1} \text { Chronic } \\
(1 \mathrm{~h} \times 7 \text { days }) \\
{ }^{2} \text { Chronic } \\
(3 \mathrm{~h} \times 21 \text { days })\end{array}$ & $\begin{array}{l}\text { Sprague- } \\
\text { Dawley } \\
\text { rats }\end{array}$ & +++ & + & $\begin{array}{c}\text { Dramatic increase } \\
\text { in CORT; increase in } \\
\text { leptin² }^{2} \text {; no changes } \\
\text { in NPY } \text { NPY }^{2}\end{array}$ & $\begin{array}{c}\text { Decrase } \\
\text { food intake } \\
\text { and body weight }\end{array}$ & $\begin{array}{l}{ }^{1} \text { Rabasa et al. (2011) } \\
{ }^{2} \text { Wang et al. (2012) }\end{array}$ \\
\hline Cold Stress & $\begin{array}{c}\text { Chronic (14 } \\
\text { days-3months) }\end{array}$ & $\begin{array}{l}\text { C57 BL6/J } \\
\text { mice }\end{array}$ & + & +++ & $\begin{array}{c}\text { Increase CORT } \\
\text { and NPY/AgRP, } \\
\text { Y2R }\end{array}$ & $\begin{array}{l}\text { Increased } \\
\text { adiposity }\end{array}$ & Kuo et al. (2007) \\
\hline
\end{tabular}

Table 1.1: Summary of stress paradigms, hormonal responses and physiological outcome. +, Mild; ++, Moderate; +++, High.

\subsection{Factors Influencing Metabolic Outcome}

One hurdle faced by those using animal models to study stress-induced obesity is choosing the right model, but also the right parameters. One of the biggest difficulties when comparing results between laboratories is that their stress paradigms are often associated with different parameters (predictability, presence/absence of a calorically dense diet, presence/absence of a recovery period, time of day, etc.). Undoubtedly, there are many variables that can be manipulated by an experimenter when using any of the aforementioned stress paradigms, all of which appear to influence the metabolic outcome of the stress paradigm. 


\subsubsection{Predictability}

Predictability of a stressor will occur when experimental routines are repeated multiple times, at the same time of day in accordance with the light/dark schedule. It is well-known that the stress response adapts quickly to the presence of a stressor (McEwen, 1998; McEwen and Wingfield, 2010), and it has therefore been suggested that unpredictability of stressors will exaggerate the normal physiological response to that stressor. Intermittent bouts of social defeat, for example, have been shown to elicit very large CORT responses (Koolhaas et al., 1997; Sgoifo et al., 1999; Moles et al., 2006) and greater increases in adipose tissue deposition in rodents when compared to repeated bouts in chronic social defeat (Foster and Solomon, 2006). Repeated bouts, on the other hand, become habituated to and elicit a dampened HPA axis response and metabolic adaptations (Keeney and Jessop, 2006). By varying the time lapse between aggressive episodes in the resident-intruder paradigm, habituation of the physiological response to the stressors cannot occur. If sustained for an extended period of time, this response may overwhelm the homeostatic mechanisms and increase the potency of that stress paradigm.

Similarly, intermittent, or acute, foot shock has been shown to cause a significant reduction in food intake and body weight, as do most physical stressors. Interestingly, when chronic foot shocks are delivered, animals are able to predict their presence, and they show no changes in caloric consumption or body weight (Griffiths et al., 1992), again lending the experimenter control over the metabolic outcome through predictability.

One must consider the evolutionary purpose of the physiological response to stress when discussing how predictability influences the outcome of a particular stressor. The physiological responses to stress are generated in attempt to meet the energetic demands of the particular stressor, liberate the appropriate energy resources while temporarily down regulating any non-pertinent bodily functions. As previously discussed, the acute stress response is an adaptive response and is one that is necessary for the survival and well-being of an organism. However, this may not be the case when dealing with protracted stressors and even more so to unpredictable protracted stressors. Habituation of the CORT response and other secreted hormones may be indicative that the demand for energy in response to the stressor may also have familiarized, thus avoiding any unnecessary changes to metabolism. However, if the animal cannot predict the presence of the stressor, then metabolic habituation is not possible which exerts the largest expense on the organism and ultimately produces the greatest metabolic consequence.

\subsubsection{Intensity}

The intensity of a stressor can be gauged through several objective measures such as glucocorticoid secretion (i.e, CORT), circulating CRF/ACTH levels, cFos activation in stress related brain regions, etc. There are limited number of studies investigating the relationship between stress severity and metabolic 
outcome, however, as a rule of thumb, the magnitude of food intake reduction as a consequence of stress can be used as a general marker of stress severity. For example, in an experiment wherein 3 types of physical stressors were used to represent low, medium and high intensity (handling, restraint, and immobilization) there was a graduated anorexic response as the intensity of the stressor increased (Martí et al., 1994). Interestingly, in this particular experiment they also employed a restraint stress (medium intensity) for different durations of time and showed no differences in reactivity, suggesting that it is in fact dependent on the stressor itself and not the length of time it is administered (Martí et al., 1994). The same pattern has been shown when comparing feeding responses between a mild food shock $(1.5 \mathrm{~mA})$ and increased CORT secretion (Rabasa et al., 2011). Similar findings have been reported from human cases, wherein eating patterns of US Marine's are negatively correlated with severity of stress exposure (Popper et al., 1989). Stress severity not only influences feeding response during times of stress, but it may also predict the persistent feeding behaviours during the recovery period (Harris et al., 1998; Vallès et al., 2000). This suggests that stress severity, even in acute stressors, may in fact shape the metabolic outcome of the stress paradigm for the long-term.

Physiologically speaking, what makes one stressor more potent then the next? Of course one would have to consider varying levels of glucocorticoid secretion, or other metabolically active hormones, given the extent to which each stressor activates CRF neurons in the PVN. However, this may not be the case. It has been shown that many different stress paradigms, both physical and psychological stressors, with seemingly different potencies elicit indiscriminate activation of the HPA axis, with measurable differences in amygdala activation (Dayas et al., 2001). Furthermore, it is well-documented that the melanocortin system is an integral component to the anorexic responses to stress. Interestingly, MC4 receptors are densely expressed in the amygdala, and when activated, these particular receptors potently inhibit food intake (Liu et al., 2013). Therefore, perhaps analysis of regional activation, outside of the HPA axis, in areas like the amygdala can better predict the intensity of a stressor and therefore the metabolic outcome. More experiments need to be conducted to better understand this phenomena.

\subsubsection{Gender Differences}

Like many other disorders (e.g. schizophrenia, major depression, anxiety, etc.) gender seems to play a key role in determining the overarching consequences of stress on body weight management. While there exists evidence suggesting that, in humans, there is no sex difference in stress exposure (Young and Korszun, 2010), there also exists evidence suggesting that women are more likely to acknowledge and/or recognize an event as being stressful (Goldman et al., 2005). In humans, women often report more stress-related eating (Greeno and Wing, 1994) and more consumption of foods high in fats and sugars in response to stressful situations (Wansink et al., 2003) compared to men. In support of these data, it has been suggested that perhaps the feeding responses to stres- 
sors is different between men and women as a result of their coping strategies (Grunberg and Straub, 1992). Men, for example, tend to display the typical fight or flight response to a stressor while women are more likely to seek out social support following stress, a strategy termed "tend-befriend" (Taylor et al., 2000).

Once again, we look to the use of animal models to further clarify the role of gender in stress induced metabolic dysfunctions. Under basal conditions, female rats show higher levels of CORT then do males (Stöhr et al., 2000) which sets the stage for divergent phenotypes in response to stress. There is an abundance of evidence demonstrating gender-specific effects of social defeat stress (Haller et al., 1999), social instability stress (Haller et al., 1999), chronic mild stress (Stöhr et al., 2000; Dalla et al., 2005; Shors et al., 2007), foot shock (IwasakiSekino et al., 2009), acute forced swim test and the development of learned helplessness (Shors et al., 2007; ?; Dalla et al., 2010). There is very limited data pertaining to the effect of social stressors on females. In the context of social defeat, males seem to be more vulnerable to changes in body weight and food intake then do females (Haller et al., 1999).It is possible that the aggressive nature of males renders them more susceptible to detrimental effects of social stressors with an aggressive component. In comparison, social stressors that lack aggression (e.g. overcrowded housing or social isolation, unpredictable social environments) elicit a greater response in female rats compared to males (Haller et al., 1999; Schmidt et al., 2010).

Given that, in humans, women are twice as likely to develop major depression then men (Kendler et al., 2002; Marcus et al., 2005), the majority of animal work done investigating gender differences in response to stress have employed the use of CMS and acute physical stressor due to their superiority in modelling human depression. It has been shown that both CMS and acute foot shock elicits an exaggerated CORT response in females compared to males (Dalla et al., 2005; Iwasaki-Sekino et al., 2009), suggesting that gender affects stress-related regions of the brain. Additionally, female rats show disruption of estrous cycles, increased floating time in a forced swim test, no reduction of body weight and dramatic changes in hippocampal serotonergic and dopaminergic neurotransmission compared to male rats, in response to CMS (Dalla et al., 2005). Interestingly, these data reflect, to some extent, human conditions of depression wherein hypo-serotonergic function in the hippocampus may lead to depressive like symptoms and correction with antidepressants, whom increase serotonergic neurotransmission, can alleviate atrophy seen in hippocampal neurons. Here we see that female rats showed reduced hippocampal serotonin turnover in response to CMS, whereas males showed no changes (Dalla et al., 2005). As would be expected, these same female rats show reduced locomotion in an open field test and increased immobility in a forced swim test (Dalla et al., 2005), behaviours reminiscent of human depression. 


\subsubsection{Genetics \& Genetic Models}

It is not surprising that genetics also contribute to the metabolic outcome of some stress paradigms. When discussing rodent models of stress, different rodent strains will reliably acquire distinct metabolic phenotypes in response to equivalent stressors. It is well-documented that different strains of rodents show different effects of social stress (Berton et al., 1998) and unpredictable chronic mild stressors including both physical and psychological components (Stöhr et al., 2000; Mineur et al., 2003; Pothion et al., 2004; Yalcin et al., 2008). Under basal conditions, for example, Fischer rats show a hyperactive HPA axis, as evidenced by elevated CORT levels, compared to the hypoactive HPA axis of the Lewis rat (Stöhr et al., 2000). This provides a unique opportunity to investigate the relationship between HPA axis activity and metabolic outcome of stress paradigms. For example, it has been shown that Fischer rats are more susceptible to the anxiogenic effects of CMS compared to Lewis rats, as would be expected based on their genetic differences (Stöhr et al., 2000). Fischer rats also demonstrate differences in their peak CORT response and negative feedback loops associated with HPA axis as measured by a two fold increase in latency to return to basal CORT levels following a stressor (Stöhr et al., 2000). Interestingly, in this study Lewis rats showed greater startle responses and more risk assessment behaviours as compared to Fischer rats (Stöhr et al., 2000), an effect thought to be mediated by CRF.

The same phenomena has been demonstrated in different mouse strains, wherein $\mathrm{BALB} / \mathrm{c}$ and $\mathrm{C} 3 \mathrm{H} / \mathrm{He}$ strains appear more sensitive to an unpredictable CMS compared to C57BL/6J mice, for example (Mineur et al., 2003). Similarly, differences in body weight changes, sucrose consumption, water maze performance, grooming behaviours, coat characteristics and responses to forced swim following unpredictable CMS vary significantly over different strains of mice (Mineur et al., 2003; Pothion et al., 2004; Yalcin et al., 2008). In a study by Pothion et al. (2004), it was demonstrated that sucrose preference over water, and baseline body weights, were consistent across 11 different strains of mice. When exposed to an unpredictable CMS, however, some obvious strain differences were observed wherein stressed mice consumed significantly less sucrose $(\mathrm{CBA} / \mathrm{H}$ and $\mathrm{C} 57 \mathrm{BL} / 6 \mathrm{~J})$ and gained significantly less weight $(\mathrm{CBA} / \mathrm{H}$ only) relative to control mice, a phenomena that did no occur in any other strain (Pothion et al., 2004). Similarly, others have shown a reduction in sucrose consumption in $\mathrm{CD}-1, \mathrm{DBA} / 2 \mathrm{~J}, \mathrm{C} 57 \mathrm{BL} 6 / \mathrm{J}$ and BALB/C mice in response to acute foot shock while this effect was entirely absent in A/J mice (Griffiths et al., 1992). Furthermore, the extent of sucrose consumption decrease varied across different strains of mice where CD-1, DBA/2J and BALB/C mice showed a greater reduction compared to that of $\mathrm{C} 57 \mathrm{BL} / 6 \mathrm{~J}$ and $\mathrm{A} / \mathrm{J}$ mice (Griffiths et al., 1992).

In addition to comparing how different rodents strains respond to a variety of stress paradigms, many have used knock-out models, wherein the gene encoding a specific peptide or receptor has been removed, to explore the roles and contributions of these peptide signalling systems in response to stress. The use 
of ghrelin and ghrelin receptor knockout mice has been instrumental in determining the role of central ghrelin in mediating the orexigenic, adipogenic and anxiolytic effects of ghrelin following chronic social defeat stress (Lutter and Sakata, 2008). Others have used the well-defined leptin deficient ob/ob mouse model to determine leptin's role in stress-induced feeding and anxiety. The obese-like phenotype in ob/ob mice is characterized by hypercorticosteronemia and leptin injections given to ob/ob mice reverse this hormonal profile (Ahima et al., 1996) suggesting that leptin plays a key role in the regulation of the HPA axis. It should be of no surprise that knockout models that interrupt normal ghrelin and/or leptin signalling will influence the metabolic response to a stressor given the dense expression of ghrelin and leptin receptors on neurons containing NPY/AgRP, POMC/CART, CRF, ACTH and more. Similarly, urocortin knockout animals have been used to help characterize the role of urocortins in mediating the physiological response to stress. Urocortin-2 deficient mice, for example, show elevations in basal stress hormones (i.e. ACTH) and do not show a rebound feeding in response to a 2 hour caloric restriction test (Chen et al., 2006). Furthermore, urocortin-2 deficient mice display an antidepressant-like phenotype when exposed to both a forced swim test and a tail-suspension test (Chen et al., 2006). CRF2 receptor deficient mice also show premature inactivation of the HPA axis (Coste et al., 2000), both illustrating urocortin's influence on HPA axis activation as well as stress induced food intake and anxiety (Coste et al., 2000; Chen et al., 2006). The same pattern of diverse physiological responses can be expected from other rodent models wherein the normal expression of hypothalamic peptides is interrupted genetically.

Interestingly, the responses to antidepressants can also vary across different rodent strains and genetically modified rodent models (Yalcin et al., 2008) suggesting not only a genetic component to stress susceptibility but to the treatment of stress-related disorders as well. For example, it has been shown that hypothalamic orexigenic peptides such as ghrelin and orexin mediate the depressive-like symptoms and antidepressant-like effects of calorie restriction following chronic stress (Lutter and Sakata, 2008; Lutter et al., 2008). These findings are relevant given the association between stress, depression and feeding. Calorically restricted orexin-null mice, for example, show anxiety like behaviours following chronic social defeat where wild-type litter mates do not (Lutter et al., 2008). Similarly ghrelin is capable of dampening the depressive like symptoms that normally arise following chronic social defeat (Lutter and Sakata, 2008). These data highlight the ability of hypothalamic feeding circuitry to influence the physiological response to stress and alter the behavioural and metabolic responses generated.

\subsubsection{Conclusion}

It has been well-established that stressful environments are conducive to the development of obesity and related metabolic disorders. Indeed, the stress response represents a metabolic challenge that is followed by physiological and behavioural responses in attempt to meet this challenge. Evolutionary pres- 
sures have shaped these homeostatic and allostatic responses, yet continued exposure to stressors and the constant struggle to meet these energetic demands may ultimately serve as detrimental. Chronic stress may therefore scale toward an increase in energy consumption and adiposity. A detailed understanding of the interaction between the stress response, the nature of the stressor and their impact on food intake and energy balance regulation may allow for more personalized treatments of obesity, be it pharmacologically of psychologically based. The use of animal models will certainly increase our understanding of these types of interactions and aid in defining the neurobiological underpinnings of consequent metabolic disorders. However, a comprehensive understanding of the wide array of stress paradigms poses its own challenges. Using variants, or derivatives, of established stress paradigms makes any type of direct comparison of data between laboratories difficult. As a result, many different stressors are broadly categorized (e.g. Table 1.1) and may not reflect the physiological differences between them. Perhaps an attempt can be made to better distinguish the details surrounding these stress paradigms or implement some type of standardization. One thing is for certain, however, in that many different stressors pose distinctive energetic challenges on an organism, and while metabolic processes are adjusted to compensate for these challenges, many result in the conservation of adipose tissue.

\subsection{Hypothetical Statement}

The contribution of metabolic hormones to the physiological response to chronic stress remains to be fully understood. What is known, however, is that stressinduced glucocorticoid secretion cannot not fully explain the metabolic outcomes of chronic stress exposure. Therefore, treatment of stress-induced obesity cannot encompass all relevant factors until the roles of other hormones are adequately defined. Ghrelin has recently emerged as a hormone that is involved in the physiological response to stress and one whose actions may explain the changes in metabolism that occur following exposure to a stressor. Given that stress has been shown to stimulate the secretion of ghrelin in the periphery, and that ghrelin is a known orexigenic and adipogenic hormone, the present thesis aims to better define the role of ghrelin in mediating the metabolic consequences of stress. The following chapters will outline and explain a series of experiments aimed at characterizing the role of ghrelin in this process and more importantly investigate the metabolic outcome of stressors in the absence of ghrelin signalling. Here, we hypothesize that stress-induced ghrelin secretion promotes an increase in appetite and a preservation of adipose tissue ultimately leading to the development of an obese-like state in rodents. Furthermore, we hypothesize that interruption of ghrelin signalling may alleviate some of these metabolic consequences. Ultimately, the data gathered in the present thesis will contribute to the knowledge of how stress-induced hormone secretion can be used as pharmacological targets for the prevention of stress-induced obesity. 


\title{
Chapter 2
}

\section{Altered Metabolic \& Neurochemical Responses to Chronic Unpredictable Stressors in Ghrelin Receptor-Deficient Mice}

\author{
Z.R. Patterson, R. Ducharme, H. Anisman \&3 A. Abizaid \\ European Journal of Neuroscience, 2010 \\ 32(4):632-9. doi:10.1111/j.1460-9568.2010.07310.x
}

\subsection{Specific Aim 1}

It has been previously demonstrated that ghrelin is secreted in response to stress (Asakawa et al., 2001b; Giordano et al., 2006; Kristenssson et al., 2006; Lutter and Sakata, 2008) and that stress-induced ghrelin secretion mediates the behavioural symptoms that arise following chronic stress (Lutter and Sakata, 2008). In addition to behavioural modifications, it is well known that chronic stress has widespread consequences on metabolism, often leading to an obese like state in both rodents and humans (McEwen, 1998; Blanchard and Spencer, 1995; Foster and Solomon, 2006; Solomon et al., 2007). With the idea that stress elicits changes in circulating hormones and results in metabolic changes, we hypothesized that stress induced ghrelin secretion may mediate the metabolic alterations observed following chronic stress. To this end, we exposed WT and GHSR KO mice to a chronic unpredictable stress paradigm and monitored their metabolic changes. 


\subsection{Introduction}

Stress is viewed as a state in which behavioral and physiological responses are generated in the face of a perceived threat. The physiological signatures of this state are sympathetic nervous system and hypothalamic-pituitary adrenal (HPA) axis activation as well as variations of several neurotransmitters within the brain (McEwen, 2007; Ulrich-Lai and Herman, 2009). Acutely, these responses are considered to be adaptive, but continuous stressor exposure may lead to physiological alterations that ultimately result in detrimental health effects, including major depressive illness, type II diabetes, obesity and cardiovascular disease (Frasure-Smith and Lespérance, 2005; Black, 2006; McEwen, 2007; Anisman et al., 2008).

A physiological consequence of chronic stress is a change in metabolism that entails an increase of metabolic rate, where proteins become inappropriately metabolized while fat stores are spared (Depke et al., 2008). These stressorinduced metabolic changes are generally attributed to continuous stimulation of both the sympathetic system and the HPA axis (Dallman and Warne, 2007; Kuo and Czarnecka, 2008). Persistent increases in glucocorticoids have, in particular, been linked to metabolic and behavioral abnormalities including obesity, compromised immunity and depression (Black, 2006; Dallman et al., 1993; Dallman and Pecoraro, 2003; Dallman and Warne, 2007; Bartolomucci et al., 2009).

Ghrelin, a hormone produced by the stomach, is associated with regulation of appetite and metabolism (Kojima et al., 1999; Tschöp et al., 2000; Nakazato et al., 2001; Kirchner et al., 2009). Continuous peripheral or central ghrelin infusions increases food intake and body weight, and favours the utilization of carbohydrates while preventing the utilizaiton of body fat as a fuel substrate, ultimately leading to increased adiposity (Tschöp et al., 2000). This would be helpful during stress, where defensive responses require the availability of energy from substrates that can be oxidized rapidly, while maintaining fat depots (Kyrou and Tsigos, 2007). Indeed, in parallel with corticosterone, circulating plasma ghrelin levels increase in response to acute and chronic stressors (Asakawa et al., 2001a; Kristenssson et al., 2006; Ochi et al., 2008; Zheng et al., 2009). Ghrelin also affects the activity of ascending monoaminergic neurotransmitter systems that are activated in response to stressors (Brunetti et al., 2002; Date et al., 2006; Kawakami, 2008; Nonogaki et al., 2006). Indeed, noradrenergic cells in the brainstem nucleus of the solitary tract (NTS), dopaminergic cells in the midbrain ventral tegmentum area and substantia niga (VTA/SN), and serotonergic cells in the midbrain raphe nucleus are all sensitive to ghrelin (Guan et al., 1997; Mitchell et al., 2001; Carlini et al., 2004; Abizaid and Liu, 2006; Date et al., 2006; Zigman and Jones, 2006) and modulate the activity of hypothalamic and limbic structures associated with stressor-induced pathology (Anisman and Zacharko, 1990; Hoebel et al., 1989; Bremner et al., 1996).

Given the links between stressors and both ghrelin and central monoamine variations, we assessed the importance of ghrelin in mediating stressor-provoked metabolic and neurotransmitter changes. To do this, we exposed mice with genetic deletions to the ghrelin receptor gene (GHSR KO) to chronic unpre- 
dictable stressors for 2 weeks (Tannenbaum et al., 2002), and compared their caloric intake, body weight gain and adipose tissue size with those of their wild-type (WT) littermates. We also examined differences in the content of norepinephrine, dopamine and serotonin and their metabolites in several brain regions affected directly or indirectly by ghrelin and by stress.

\subsection{Materials \& Methods}

\subsubsection{Animals}

Male GHSR WT $(\mathrm{n}=20)$ and male GHSR KO $(\mathrm{n}=20)$ mice, 2-4 months old that had been bred at Carleton Universitys Institute of Neuroscience, served as experimental subjects. These mice were created on a C57BLJ6 and DBA mixed background strain and originated from breeding pairs obtained from Regeneron Pharmaceuticals Inc. (Tarrytown, NY, USA). Their metabolic phenotype has previously been characterized (Pfluger et al., 2008). All animals were singlehoused in standard plastic mouse cages $(27 \times 21 \times 14 \mathrm{~cm})$ in a temperaturecontrolled room $\left(22^{\circ} \mathrm{C}\right)$, and kept on a twelve hour lightdark cycle with lights on at 07:00 $\mathrm{h}$ for the duration of the study. Stressed animals were housed in a different room from non-stressed animals. All procedures were approved by the

Carleton University Animal Care Committee and followed the guidelines of the Canadian Council on Animal Care.

\subsubsection{Procedure}

Animals were matched for age (average age $82.8 \pm 2.3$ days old) and body weight $(30.1 \pm 1.13 \mathrm{~g})$ and were assigned to one of four groups: (i) WT no stress $(\mathrm{n}=10)$, (ii) WT stress $(\mathrm{n}=10)$, (iii) KO no stress $(\mathrm{n}=10)$ and (iv) KO stress $(\mathrm{n}=10)$. Standard and high-fat diet intake and body weight were measured for 10 days before the onset of the unpredictable stressor regimen to obtain a baseline. At the end of the baseline period, WT and GHSR KO mice in the stress groups were taken to a separate room and subjected to two daily stressors for 14 days. The stressors used were as follows: (i) Restraint: mice were placed in a semicircular chamber strainer made of clear Plexiglas $(4.0 \mathrm{~cm}$ diameter $\times 12.0 \mathrm{~cm}$ long), for 15 minutes with their tails taped to prevent them from turning. (ii) Exposure to predator scent: mice were placed in a rat cage $(32 \times 22 \times 20 \mathrm{~cm})$ with soiled litter for 15 minutes. (iii) Novel aversive environment: mice were placed in a clear mouse cage $(27 \times 21 \times 14 \mathrm{~cm})$ with wet bedding for 15 minutes. (iv) Social stressor: mice were placed in the cage of a sexually experienced male mouse (a mouse of the highly aggressive CD-1 strain) for a maximum of 15 minutes, or until fighting occurred. This interaction was always stopped before experimental mice were physically injured. (v) Forced swim test: mice were placed in a pool of water $(32 \times 22 \times 20 \mathrm{~cm})$ at $20^{\circ} \mathrm{C}$ for 3 minutes. (vi) Noise stress: mice were kept in their home cages and exposed to loud music for 15 minutes. (vii) Open field stress: mice were placed 
in the center of an open field box $(60 \times 60 \times 20 \mathrm{~cm})$ for a period of 15 minutes. Animals were exposed to a different stressor (chosen randomly) and at two randomly selected times of the day. In this way, mice were not able to adapt to a single stressor nor were they able to predict when they were to be stressed. This combined stressor paradigm has been used previously and shown to be effective in producing marked hormonal and neurochemical responses (Shanks et al., 1994; Mineur et al., 2003, 2006, 2007). We emphasize that this regimen involves stressors that are more intense than those typically used in chronic mild stress paradigms (Willner et al., 1987). Following the 14th day of treatment, mice from both groups were killed by rapid decapitation 5 minutes after the last stressor (this being 15 minutes of restraint stress). Control non-stressed mice were killed on the same day as stressed animals, and occurred between 08:00 and 12:00 h. Trunk blood was collected in borosilicate tubes coated with EDTA and containing $10 \mu \mathrm{L}$ of $1 \mathrm{~N} \mathrm{HCl}$ per millilitre of blood and a cocktail of protease inhibitors and chilled on ice before being centrifuged. Brains were rapidly dissected and placed on a stainless steel block (immersed in ice) with slots (spaced approximately $500 \mu \mathrm{m}$ apart) that served as guides for razor blades. Tissue micropunches of the hippocampus (1.5 - 3.0mm behind bregma), central nucleus of the amygdala (1.0 - 1.5mm behind bregma), paraventricular nucleus of the hypothalamus (PVN) (0.5 - $1.0 \mathrm{~mm}$ behind bregma), arcuate nucleus of the hypothalamus (ARC) (1.5 - 2.0mm behind bregma), prefrontal cortex (1.0 - $1.5 \mathrm{~mm}$ in front of bregma), locus coeruleus (5.3 - 5.8mm behind bregma) and the nucleus accumbens $(1.0-1.5 \mathrm{~mm}$ in front of bregma) were collected from slices using hollow 16- and 20-gauge needles with a beveled tip as described by Palkovits (1973). Paxinos \& Franklin (2001) was used as a guide. Tissue punches were placed in $0.3 \mathrm{M}$ monochloroacetic acid containing $10 \%$ methanol and internal standards, and stored at $-80^{\circ} \mathrm{C}$ until assayed. Following decapitation, mice bodies were collected, wrapped in aluminum foil and frozen at $-80^{\circ} \mathrm{C}$ until fat dissection was possible. The carcasses were later thawed and retroperitoneal, perigonadal and subcutaneous fat pads were dissected and weighed. The mass of adipose tissue in these fat pads was converted to a percentage of total body weight for statistical analyses.

\subsubsection{Caloric Intake \& Body Weight}

Mice were given free access to standard laboratory chow (18\% caloric content from fat; $3.0 \mathrm{kcal} / \mathrm{g}$; Harlan Diets, Misississaga, ON, Canada), a high-fat diet (58\% caloric content from fat, 5.56 kcalg; Research Diets, New Brunswick, NJ, USA) and tap water throughout the study. Measurements of weight and caloric consumption for both standard lab diet and high-fat diet were recorded over a 10-day baseline period and during a 14-day stressor period. Recordings were carried out at the same time every day. Caloric value for each food type was used to calculate the total daily caloric intake for each mouse across both the baseline and the treatment periods, as well as the percentage of total calories consumed coming from each food type. Caloric efficiency, defined as the amount of weight gained per calorie of energy consumed during a given time period, was 
calculated for each mouse during the baseline period as well as the treatment period.

\subsubsection{Hormone Level Analysis}

Blood samples were centrifuged at $3000 \times \mathrm{g}$ for 15 minutes at $4^{\circ} \mathrm{C}$ to separate plasma from red blood cells. The plasma was then collected and stored at $-20^{\circ} \mathrm{C}$. Plasma acylated ghrelin and corticosterone levels were measured in duplicate using commercially available radioimmunoassay (RIA) kits (ICN Biomedicals, CA, USA), sensitivity ranging from 5.0 to $40.0 \mu \mathrm{g} / \mathrm{dL}$; acylated ghrelin (Millipore, MA, USA), sensitivity ranging from 7.9 to $1000 \mathrm{pg} / \mathrm{mL}$. The RIA assay for acylated ghrelin detects with $100 \%$ cross-reactivity to the active form of ghrelin (acylated ghrelin) in plasma samples from humans, rodents and canine species. Furthermore, the antibody detects specifically the first three aminoacids of the ghrelin molecule, including the Octanoyl group that is acylated to the serine 3 aminoacid in the sequence. This particular portion is shared by most vertebrates (Hosoda et al., 2006). All samples were assayed in a single run for each hormone and had an inter-assay variability lower than $10 \%$.

\subsubsection{Brain Monoamine Analysis}

Brain samples from all regions described above were homogenized and monoamine neurotransmitter analyses were performed from each independent sample using high-performance liquid chromatography (HPLC), as previously described (Anisman and Zacharko, 1990). Levels of dopamine (DA), norepinephrine (NE) and serotonin (5-HT), and their metabolites, 3,4-dihydroxy-phenylacetic acid (DOPAC), 3-methoxy-4-hydroxyphenylglycol (MHPG) and 5-hydroxyindolacetic acid (5-HIAA), respectively, were assessed in each of the brain regions. To this end, tissue punches were sonicated in a homogenizing solution comprising $14.17 \mathrm{~g}$ monochloroacetic acid, $0.0186 \mathrm{~g}$ EDTA, $5.0 \mathrm{ml}$ methanol and $500 \mathrm{~mL}$ HPLC-grade water. Following centrifugation, supernatants were used for the HPLC analysis. Using an Agilent pump (Mississauga, Ontario, Canada), guard column, radial compression column $(5 \mathrm{~m}, \mathrm{C} 18$ reverse phase, $8 \mathrm{~mm} \times 10 \mathrm{~cm})$, and colometric electrochemical detector (ESA), $40 \mu \mathrm{l}$ of the supernatant from each individual region sampled from each mouse was passed through the system at a flow rate of $1.5 \mathrm{~mL} / \mathrm{min}$ (1400 - 1600 pounds per square inch). Each litre of mobile phase contained sodium dihydrogen phosphate $(90 \mathrm{~mm})$, 1-octase sulfonic acid $(1.7 \mathrm{~mm})$, EDTA $(50 \mathrm{~mm})$, citric acid $(50 \mathrm{~mm})$, potassium chloride $(5 \mathrm{~mm})$ and $10 \%$ acetonitrile. The mobile phase was filtered $(0.22 \mathrm{~mm}$ filter paper) and degassed. The area and height of the peaks were determined using an Agilent data integrator and the software associated with this instrument. The protein content of each sample was determined using bicinchoninic acid with a protein analysis kit (Pierce Scientific, Brockville, ON, Canada) and a Fluorostar colorimeter (BMG, Durham, NC, USA). The lower limit of detection for the monoamines and metabolites was approximately $1.0 \mathrm{pg}$. 


\subsubsection{Statistical Analysis}

All data were analysed using $2 \times 2$ ANOVA with genotype (KO and WT) and treatment (stress or no stress) as the between-group factors. Tukeys HSD post hoc tests were performed where significant effects were found. The limit for statistical significance was set at an $\alpha=0.05$.

\subsection{Results}

\subsubsection{GHSR KO Mice Do Not Show the Stressor-Induced Alterations in Caloric Intake \& Body Weight Seen in GHSR WT mice}

Figure 2.1 shows the mean change in caloric intake during the treatment period as compared with baseline. The chronic stressor caused a significant decrease in caloric intake in WT stressed animals, but a similar effect was not observed in GHSR KO stressed mice [significant treatment $\times$ genotype interaction $\left(\mathrm{F}_{(1,34)}=\right.$ $9.16, p<.05)$ ]. Similarly, body weight gain and caloric efficiency were lowered in WT stressed but not in GHSR KO stress mice [significant treatment $\times$ genotype interaction (weight gain: $\mathrm{F}_{(1,34)}=5.86, p<.05$; caloric efficiency: $\mathrm{F}_{(1,34)}=8.48$, $p<.05)$ ]. Interestingly, there were no selective differences in the proportion of calories obtained from either the palatable high-fat diet or the standard chow $(p$ $>$.05), suggesting that the drop in caloric intake seen in WT mice comes from a drop in the consumption of both diets. Figure 2.2 shows the proportion of body weight stored as fat in different fat pads. Despite a significant decrease in caloric intake, WT mice had an overall larger proportion of body weight stored in the fat pads than did GHSR KO mice (main effect of genotype, $\mathrm{F}_{(1,34)}=94.03, p<$ $.05)$. Thus, WT mice maintained their total percentage of body fat and tended to have the highest percentage of abdominal fat [retroperitoneal + perigonadal, main effect of genotype $\left.\mathrm{F}_{(1,34)} 101.09, p<.05\right)$ ]. Similarly, WT mice tended to have more abdominal fat in relation to body weight than did GHSR KO mice and the stressor did not affect this proportion [main effect of genotype $\left.\left.\mathrm{F}_{(1,34)}=56.97, p<.05\right)\right]$. Analysis of subcutaneous fat indicated that WT mice had higher proportion of subcutaneous fat than GHSR KO mice (Figure $\left.2.2 \mathrm{C} ; \mathrm{F}_{(1,34)}=56.97, p<.05\right)$, but stress had no overall effect on the proportion of subcutaneous fat $\left(\mathrm{F}_{(1,34)}=0.296, p=.0591\right)$, and did not affect WT GHSR $\mathrm{KO}$ mice differentially (stress $\times$ genotype interaction, $\mathrm{F}_{(1,34)}=.154, p=.699$ ). Figure 2.3 shows the plasma ghrelin and corticosterone levels measured from trunk blood samples taken at the time of death. The analyses revealed that stressed mice had significantly higher plasma active ghrelin $\left(\mathrm{F}_{(1,34)}=5.04, p\right.$ $<.05)$ and corticosterone levels $\left(\mathrm{F}_{(1,34)}=194.56, p<.001\right)$. Moreover, WT animals showed overall higher levels of corticosterone then did KO mice $\left(\mathrm{F}_{(1,34)}\right.$ $=5.56, p<.05)$. 


\subsubsection{Aminergic Neurotransmitter Concentrations are Dif- ferentially Altered by Stress in GHSR KO Mice}

Table 2.1 shows a summary of the significant main effects detected by the ANOVAs examining brain differences in the content of aminergic neurotransmitters and their metabolites in stressed and non-stressed GHSR KO and WT mice. All interaction effects are described in detail below. Non-significant interactions effects $(p>.05)$ are not shown.

NE \& MHPG Statistical analyses showed that total NE concentrations were not significantly affected by the chronic stressor paradigm in any of the regions examined $(p>.05)$. The chronic stressor, however, did increase the accumulation of MHPG in the central nucleus of the amygdala, hippocampus, prefrontal cortex, and the hypothalamic PVN (see Table 2.1). Within the prefrontal cortex the stress-induced increase in MHPG was greater in GHSR KO mice than in WT mice as shown by a significant interaction effect $\left(\mathrm{F}_{(1,34)}=\right.$ 4.56 and 5.22, $p<.05$; Figure 2.4). Interestingly, MHPG concentrations in the ARC of the stressed GHSR WT mice were lower than those in non-stressed WT mice, whereas in the ARC of stressed GHSR KO mice MHPG concentrations were increased (significant interaction effect, $\mathrm{F}_{(1,34)}=5.22, p<.05$; Figure 2.4).

DA, HVA \& DOPAC Overall, the chronic stressor paradigm was effective in altering DA utilization in some of the regions examined, as reflected by increases in the levels of DA metabolites but not DA. Specifically, the stressor increased the DA content in the nucleus accumbens (main effect of stress: $\mathrm{F}_{(1,34)}$ $=3.98, p=.05)$, and also increased DOPAC accumulation $\left(\mathrm{F}_{(1,34)}=7.97, p<\right.$ $.05)$. Interestingly, the stressor increased DOPAC concentrations in WT mice but not in GHSR KO mice (significant genotype $\times$ stress interaction, $\mathrm{F}_{(1,34)}$ $=5.19, p .05$; Figure 2.5). Finally, the stressor also increased DOPAC levels in the prefrontal cortex $\left(\mathrm{F}_{(1,34)}=5.60, p<.05\right)$, but it did so to all animals irrespective of their genotype.

5-HT \& 5-HIAA 5-HT concentrations in stressed and non-stressed GHSR $\mathrm{KO}$ and WT mice were not different in any of the areas examined with the exception of the ARC, where 5-HT levels were significantly lower in GHSR WT compared with GHSR KO mice $\left(\mathrm{F}_{(1,34)}=6.55, p<.05\right)$. Concentrations of the 5-HT metabolite 5-HIAA in the amygdala, PVN and prefrontal cortex were increased in stressed mice, regardless of their genotype $\left(\mathrm{F}_{(1,34)}=7.74, p<\right.$ .05 for amygdala; $\mathrm{F}_{(1,34)}=21.55, p<.001$ for PVN; $\mathrm{F}_{(1,34)}=11.47, p<.05$ for prefrontal cortex; Table 2.1). Interestingly, regardless of the manipulations, GHSR KO mice had lower levels of 5-HIAA in the PVN than GHSR WT mice (main effect of genotype, $\mathrm{F}_{(1,34)}=4.24, p<.05$; Figure 2.6). 


\subsection{Discussion}

Stress is an expensive energetic state, and organisms respond to this challenge by mobilizing energy that is easy to break down into useable substrates. Thus, in the face of acute stress, carbohydrates are preferentially metabolized, while anabolic processes are delayed. These processes have been previously linked to the peripheral actions of glucocorticoids (Sapolsky et al., 1986; Black, 2006; Depke et al., 2008). In the present study, stressed WT mice showed an overall decrease in their total caloric intake that was independent of the type of diet the mice consumed, energy efficiency and a reduction in body weight gain similar to that reported in the literature (Depke et al., 2008). Chronically stressed GHSR $\mathrm{KO}$ mice, however, do not show these metabolic changes despite having physiologically relevant glucocorticoid responses to stressors. Therefore, our data suggest that ghrelin, like glucocorticoids, is important for the metabolic shift required to deal with chronic stress. Although levels of corticosterone were overall lower in GHSR KO mice, corticosterone responses to stress were not different between strain, making it unlikely that this hormone mediates the inter-strain changes in body weight, caloric intake and metabolic efficiency in response to stressors. The presence of glucocorticoids, however, may be necessary for ghrelin to maintain adiposity in WT stressed mice, as intact adrenals are necessary for the adipogenic effects of ghrelin mimetics (Tung et al., 2004).

In parallel with the changes in body weight, caloric intake and metabolic efficiency in response to stressors, several monoamine alterations were observed in stressed animals, some of which differed between GHSR KO and WT mice. There were few differences in the content of NE and DA and their metabolites between non-stressed GHSR KO and WT mice. Higher levels of MHPG, however, were observed in the amygdala, PVN, hippocampus and prefrontal cortex, and tended to increase in the locus coeruleus of stressed mice regardless of their genotype. This effect has been previously reported, and demonstrates that the unpredictable stress paradigm was effective in producing a significant neurochemical response in the brain (Shanks et al., 1994; Anisman and Zacharko, 1990; Tannenbaum et al., 2002; Ulrich-Lai and Herman, 2009).

The only region where NE neurotransmission was not increased overall by the stressor was the ARC. Here, MHPG content was reduced in stressed WT mice but not in the GHSR KO mice. The ARC is an important conduit for the regulation of the metabolism by peripheral signals such as ghrelin and corticosterone (Abizaid and Horvath, 2008). Cells within the ARC produce orexigenic (i.e. Neuropeptide Y (NPY) and Agouti-related peptide (AgRP)), and anorectic (cocaine-amphetamine related transcript (CART), alpha-melanin stimulating hormone $(\alpha-\mathrm{MSH}))$ peptides that regulate energy balance and these peptides are modulated by NE (Abizaid and Horvath, 2008; Wellman, 2000). Indeed, NE stimulates feeding as well as the expression of NPY and AgRP in the ARC of rats, and ghrelin's effects on food intake depend on noradrenergic inputs to the ARC (Fraley et al., 2002; Fraley and Ritter, 2003; Date et al., 2006). Lower NE utilization in the ARC could therefore explain the decrease in general caloric intake that was observed in stressed WT mice. Of course, this is speculative and 
it will be necessary to determine whether the effects of the stressor on caloric intake and body weight are modifiable by $\mathrm{NE}$ alterations at the ARC.

Overall, GHSR KO mice tended to be slightly smaller than WT mice and to accumulate less body fat despite having access to high-calorie diet, a phenotype reported by Pfluger et al. (2008). This may be explained by strain differences in serotonin content in the ARC, where serotonin levels were higher in GHSR $\mathrm{KO}$ mice than in WT mice regardless of treatment. Serotonin acts on proopiomelanocortin cells in the ARC to stimulate the release of $\alpha$-MSH, a potent stimulator of energy expenditure with anorexic properties (Heisler et al., 2002; Zhou et al., 2005). These data would support the idea that ghrelin inhibits the release of serotonin in the ARC of mice, as shown previously (Brunetti et al., 2002).

Chronic stress did increase levels of serotonin metabolites in the PVN of mice, an effect particularly evident in WT mice. Increased serotonin neurotransmission in the PVN following stress has been associated with increased production of corticotropin-releasing factor in the PVN, and with decreased food intake and body weight (Jø rgensen et al., 2002, 1998; Le Feuvre et al., 1991). Thus, it seems likely that both the overall decreases in caloric intake seen in stressed WT mice are related to stress-induced increases in serotonin utilization in the PVN that are not seen in GHSR KO mice.

In corroboration of previous research, NE utilization was increased in the prefrontal cortex of stressed mice (Anisman and Zacharko, 1990; Shanks et al., 1994; Birnbaum et al., 1999; Arnsten, 2000, 2009; Tannenbaum et al., 2002). In this instance, stressed GHSR KO mice showed greater levels of MHPG than stressed WT mice. The prefrontal cortex plays an important role in regulating HPA axis activity following acute stressors (Diorio et al., 1993). Nevertheless, although increased NE neurotransmission may lower the activity of the HPA axis, excess NE activity in the prefrontal cortex has been associated with impaired stress regulation and ultimately psychopathology (Birnbaum et al., 1999; Arnsten, 2000, 2009). These results might suggest that ghrelin secretion in response to stressors serves to limit NE release and utilization in the prefrontal cortex, and that unrestrained NE release and utilization in the prefrontal cortex may be detrimental to GHSR KO mice and render them vulnerable to stressorinduced psychopathology. Indeed, Lutter and Sakata (2008) have shown that GHSR KO mice display more depressive-like behaviors following chronic exposure to social stressors than their WT counterparts.

As shown previously (Anisman and Zacharko, 1990; Tannenbaum et al., 2002), the chronic unpredictable stressor paradigm used in the present study also resulted in increased levels of DA metabolites in the PVN, prefrontal cortex and nucleus accumbens. It is particularly interesting that in the nucleus accumbens, DA utilization was significantly increased only in WT mice. DA released into the nucleus accumbens comes primarily from cells in the midbrain VTA and is associated with reward-seeking behaviors (Wise, 2004). About 50$60 \%$ of the VTA DA cells contain ghrelin receptors and these cells are stimulated by ghrelin (Guan et al., 1997; Abizaid and Liu, 2006; Zigman and Jones, 2006). Peripheral injections and local ghrelin infusions into the VTA produce DA re- 
lease in mice and rats, an effect that is absent in GHSR KO mice (Abizaid and Liu, 2006; Jiang et al., 2006; Jerlhag et al., 2007; Jerlhag, 2008). As such, it is possible that stress-induced increases in DA release into the nucleus accumbens are mediated in part by ghrelin acting on VTA DA neurons. This conclusion is consistent with previous observations (Abizaid and Liu, 2006; Abizaid, 2009) and raises the possibility that ghrelin might contribute to the rewarding effects of dopamine in appetitive situations.

Here we used mice with genetic deletions of the GHSR gene and as such developed in the absence of this receptor. This probably caused developmental patterns that are different from those of their WT littermates, and thus it is difficult to determine if the data obtained are due to abnormal developmental patterns or to the actions of ghrelin on its receptor in adult animals. Future studies using either pharmacological blockade of the receptor or genetic knockdown of the receptor in adult may provide for more specific answers to this question. There is also the possibility that receptors other than GHSR bind ghrelin to alter adiposity (Halem et al., 2005). However, the relative contribution of these putative receptors remains obscure. Overall, our results indicate that ghrelin, a hormone normally associated with the regulation of appetite and energy balance, plays an important role in mediating the metabolic and neurochemical responses to stressors. In line with this perspective, several studies have shown that ghrelin is increased following acute and chronic stressors (Asakawa et al., 2001a; Giordano et al., 2006; Kristenssson et al., 2006; Ochi et al., 2008). Acutely, the release of ghrelin appears to enhance the activity of the HPA axis and it may be important in the regulatory feedback mechanisms controlling this endocrine response (Asakawa et al., 2001a; Kristenssson et al., 2006; Stevanović et al., 2007). Additionally, ghrelin could play a role in the allostatic changes required to respond to acute and chronic stressors. In this context, ghrelin may serve to maintain energy balance in stressed organisms by increasing the release of glucose available for utilization while protecting fat stores from depletion (Tschöp et al., 2000; van der Lely, 2009). Continuous exposure to stress may, however, unmask negative metabolic consequences that could be related to ghrelin, including hyperglycemia, increased adiposity and ultimately obesity. 
A

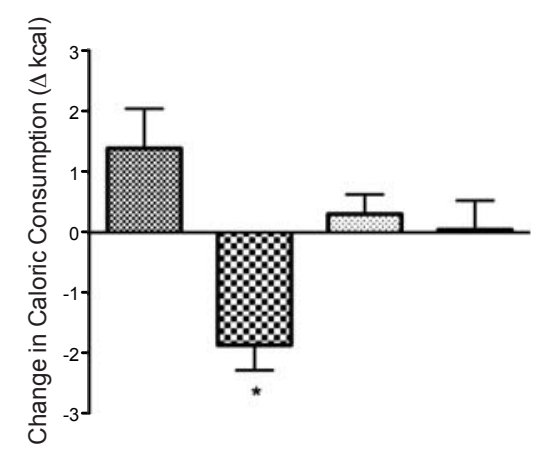

$\mathrm{C}$

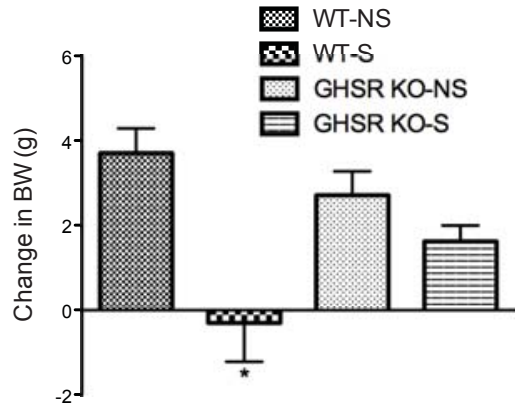

B

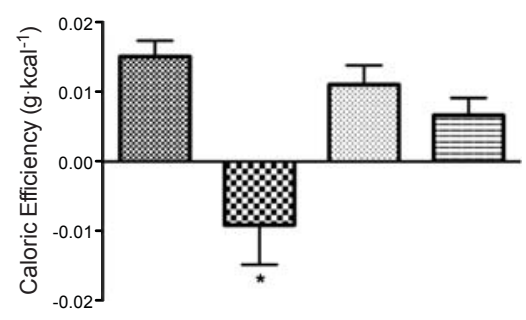

D

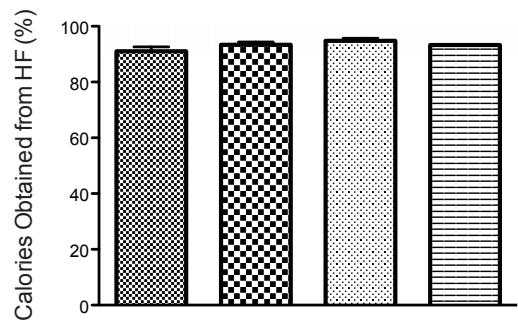

Figure 2.1: Stress-induced changes from baseline in caloric intake (A), caloric efficiency (B), weight change (C) and proportion of calories obtained from the consumption of a high-fat diet (D). All values are expressed as mean $\pm \mathrm{SEM}$. ${ }^{*} p<.05$ compared to all other groups 
A
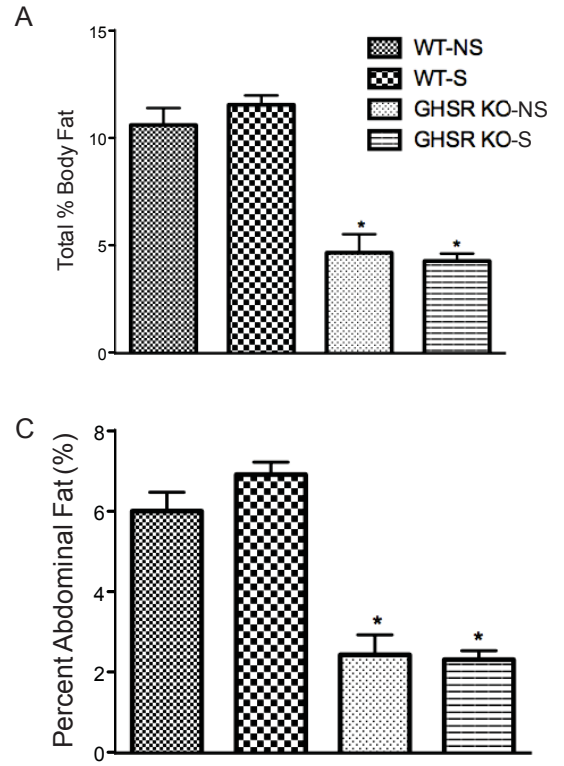

B

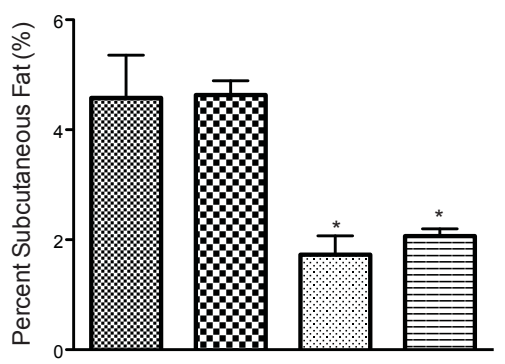

Figure 2.2: Stress-induced alterations at the end of the stress paradigm in total percentage body fat/total body weight (A), percentage of abdominal fat/total body weight (B) \& percent subcutaneous fat/total body weight (C) in nonstressed and stressed mice. All values are expressed as mean \pm SEM. ${ }^{*} p<.05$ compared to WT mice ( $\mathrm{n}=10$ per group). 

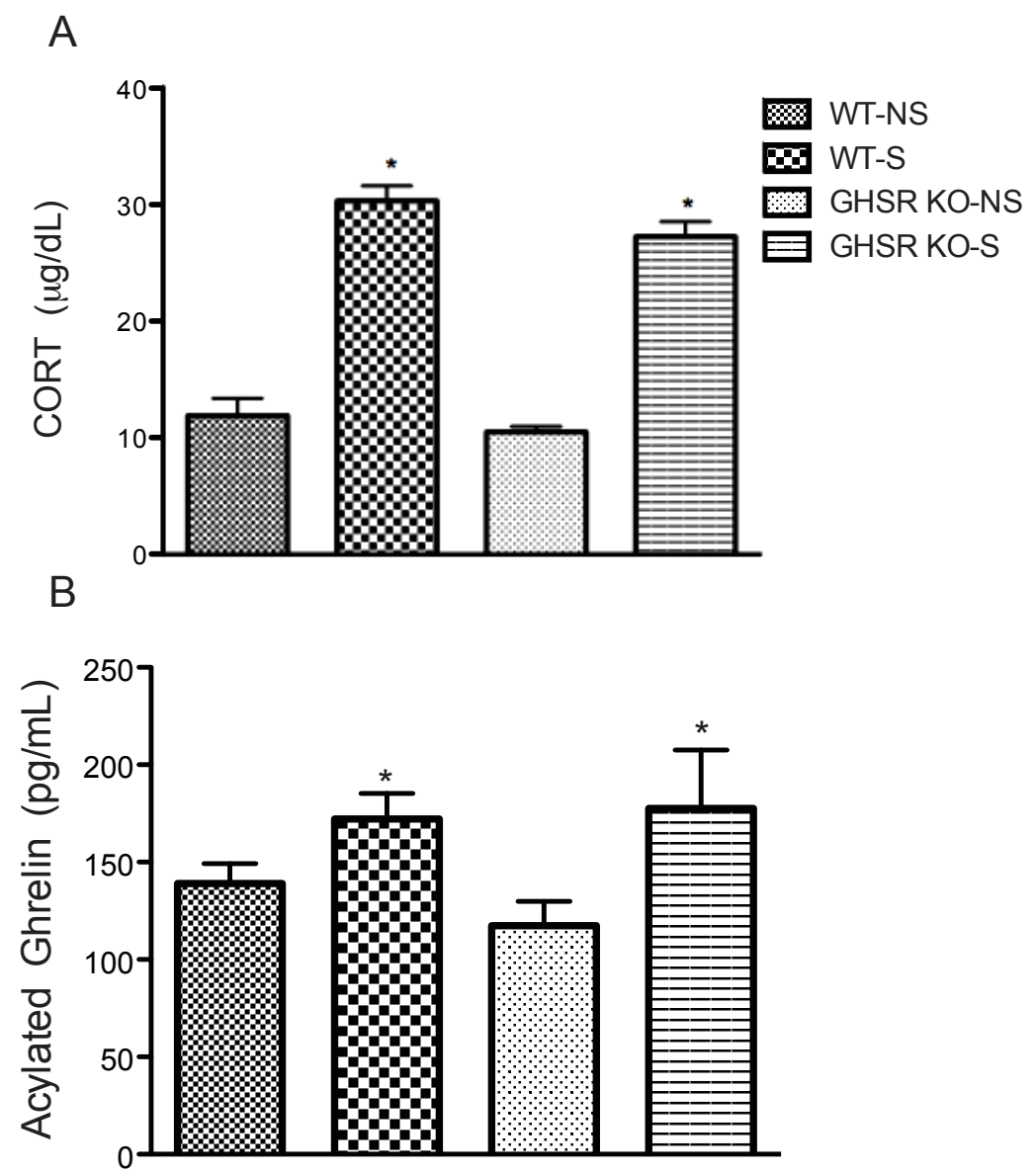

Figure 2.3: Mean levels of circulating corticosterone (A) \& mean levels of circulating acylated ghrelin (B) at the time of sacrifice in non-stressed and stressed mice. ${ }^{*}<.05$ compared to non-stressed controls $(\mathrm{n}=10$ per group) 
Prefrontal Cortex

A
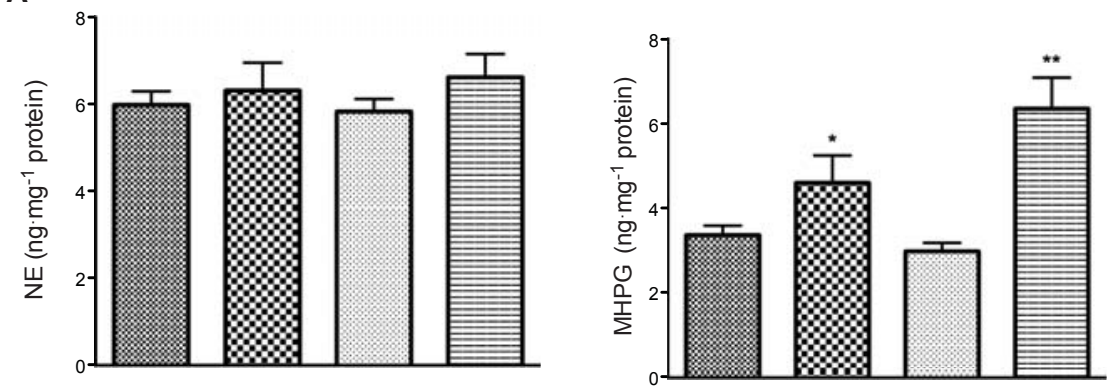

Arcuate Nucleus

B
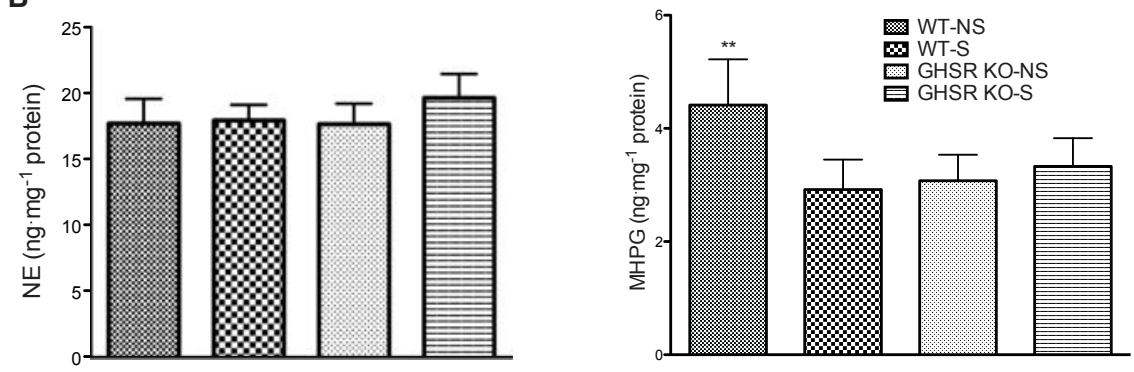

Figure 2.4: Mean levels of NE \& MHPG in the prefrontal cortex (A) and the arcuate nucleus of the hypothalamus (B) of non-stressed and stressed mice. ${ }^{*} p$ $<.05$ compared to non-stressed controls, ${ }^{* *} p<.05$ compared to all other groups.

Nucleus Accumbens
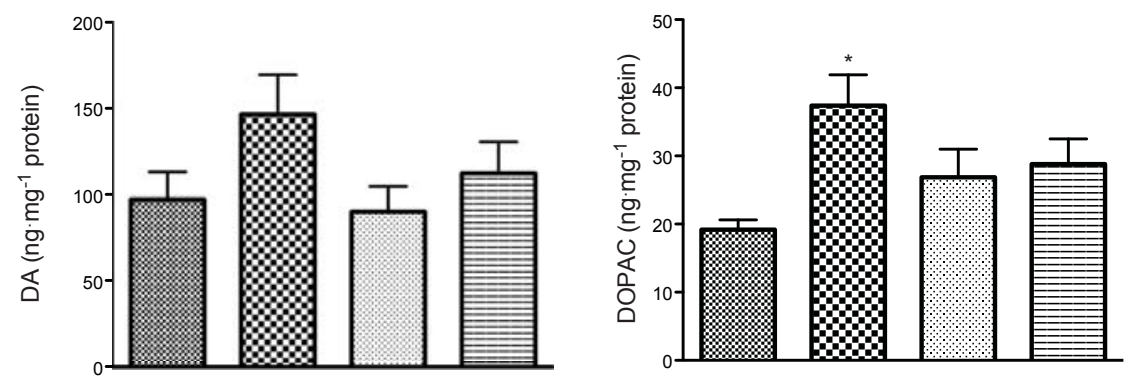

Figure 2.5: Mean levels of DA \& DOPAC in the nucleus accumbens of nonstressed and stressed mice. ${ }^{* *} p<.05$ compared to all other groups. 
A

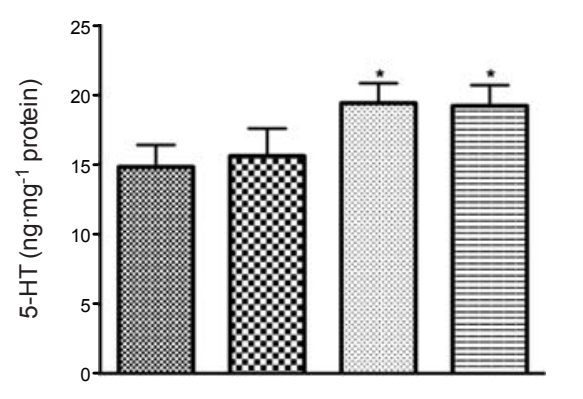

Paraventricular Nucleus

B

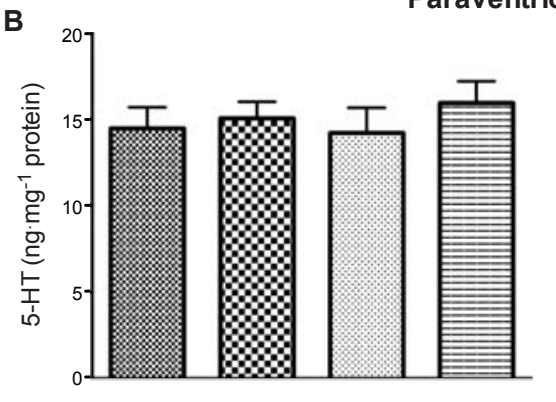

Arcuate Nucleus
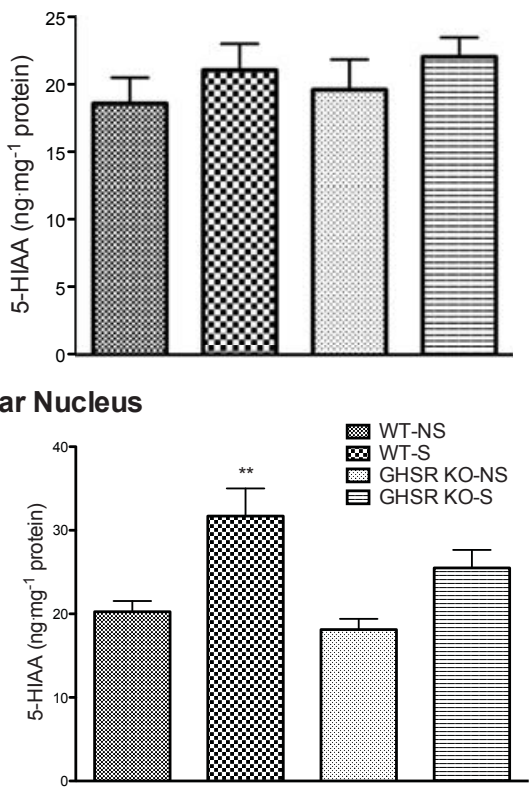

Figure 2.6: Mean levels of 5-HT \& 5-HIAA in the arcuate nucleus of the hypothalamus (A) and the paraventricular nucleus of the hypothalamus (B) of non-stressed and stressed mice. ${ }^{*} p<.05$ compared to WT stressed mice, ${ }^{* *} p$ $<.05$ compared to all other groups 


\begin{tabular}{|c|c|c|c|c|c|}
\hline & & WT Control & WT Stressed & GHSR KO Control & GHSR KO Stress \\
\hline \multicolumn{6}{|l|}{ NE \& MHPG } \\
\hline \multirow[t]{2}{*}{ Amygdala } & $\mathrm{NE}$ & $23.27 \pm 1.02$ & $24.42 \pm 0.95$ & $25.44 \pm 1.33$ & $23.97 \pm 1.39$ \\
\hline & $\mathrm{MHPG}^{*}$ & $4.84 \pm 0.64$ & $8.11 \pm 1.81$ & $4.78 \pm 0.75$ & $7.99 \pm 1.17$ \\
\hline \multirow{2}{*}{ Arcuate Nucleus } & NE & $17.67 \pm 1.87$ & $17.91 \pm 1.17$ & $17.62 \pm 1.55$ & $19.62 \pm 1.80$ \\
\hline & $\mathrm{MHPG}^{\psi}$ & $4.41 \pm 0.81$ & $2.92 \pm 0.53$ & $3.07 \pm 0.46$ & $4.33 \pm 0.50$ \\
\hline \multirow[t]{2}{*}{ Hippocampus } & $\mathrm{NE}$ & $7.16 \pm 0.52$ & $6.90 \pm 0.71$ & $8.30 \pm 0.86$ & $6.60 \pm 0.51$ \\
\hline & MHPG & $4.65 \pm 0.59$ & $5.27 \pm 0.45$ & $4.05 \pm 0.47$ & $5.92 \pm 0.99$ \\
\hline \multirow[t]{2}{*}{ Locus Coeruleus } & $\mathrm{NE}$ & $16.87 \pm 1.02$ & $17.29 \pm 1.48$ & $19.13 \pm 1.36$ & $18.68 \pm 1.40$ \\
\hline & MHPG & $3.43 \pm 0.34$ & $5.23 \pm 1.25$ & $3.71 \pm 0.34$ & $4.55 \pm 0.72$ \\
\hline \multirow[t]{2}{*}{ Prefrontal Cortex } & $\mathrm{NE}$ & $5.97 \pm 0.31$ & $6.29 \pm 0.64$ & $5.81 \pm 0.29$ & $6.60 \pm 0.54$ \\
\hline & $\mathrm{MHPG}^{\psi}$ & $3.37 \pm 0.22$ & $4.59 \pm 0.65$ & $2.99 \pm 0.20$ & $6.34 \pm 0.74$ \\
\hline \multirow[t]{2}{*}{ Paraventricular Nucleus } & $\mathrm{NE}$ & $39.31 \pm 1.37$ & $39.99 \pm 0.81$ & $37.36 \pm 2.29$ & $42.84 \pm 1.65$ \\
\hline & $\mathrm{MHPG}^{*}$ & $4.79 \pm 1.37$ & $39.99 \pm 0.81$ & $37.36 \pm 2.29$ & $42.84 \pm 1.65$ \\
\hline \multicolumn{6}{|l|}{ DA. HVA \& DOPAC } \\
\hline \multirow[t]{3}{*}{ Nucleus Accumbens } & DA & $97.23 \pm 15.56$ & $145.98 \pm 22.98$ & $90.13 \pm 14.28$ & $111.92 \pm 18.37$ \\
\hline & DOPAC $^{\psi}$ & $19.19 \pm 1.42$ & $37.40 \pm 4.50$ & $26.86 \pm 4.14$ & $28.80 \pm 3.68$ \\
\hline & HVA* & $1.87 \pm 0.32$ & $2.93 \pm 0.50$ & $1.933 \pm 0.71$ & $3.28 \pm 0.71$ \\
\hline \multirow[t]{3}{*}{ Prefrontal Cortex } & DA & $2.82 \pm 0.30$ & $2.51 \pm 0.29$ & $2.32 \pm 0.21$ & $2.71 \pm 0.23$ \\
\hline & DOPAC* & $1.92 \pm 0.20$ & $2.72 \pm 0.43$ & $1.65 \pm 0.20$ & $2.21 \pm 0.31$ \\
\hline & $\mathrm{HVA}^{\Phi}$ & $1.16 \pm 0.11$ & $1.76 \pm 0.29$ & $2.01 \pm 0.57$ & $2.97 \pm 0.69$ \\
\hline \multirow{3}{*}{ Paraventricular Nucleus } & DA & $17.13 \pm 0.81$ & $17.75 \pm 0.57$ & $15.49 \pm 1.18$ & $17.81 \pm 0.81$ \\
\hline & DOPAC & $7.02 \pm 0.29$ & $7.40 \pm 0.23$ & $6.53 \pm 0.37$ & $7.32 \pm 0.33$ \\
\hline & HVA* & $7.77 \pm 0.63$ & $13.84 \pm 1.26$ & $7.87 \pm 1.30$ & $13.55 \pm 1.25$ \\
\hline \multicolumn{6}{|l|}{ 5-HT \& 5-HIAA } \\
\hline \multirow[t]{2}{*}{ Amygdala } & 5-HT & $26.80 \pm 2.02$ & $22.76 \pm 2.90$ & $22.39 \pm 1.75$ & $26.78 \pm 3.66$ \\
\hline & 5-HIAA* & $20.55 \pm 1.35$ & $25.59 \pm 3.74$ & $18.85 \pm 1.34$ & $27.49 \pm 3.03$ \\
\hline \multirow[t]{2}{*}{ Arcuate Nucleus } & 5 -HT* & $14.83 \pm 1.57$ & $15.60 \pm 1.99$ & $19.42 \pm 1.43$ & $19.23 \pm 1.48$ \\
\hline & 5-HIAA & $18.56 \pm 1.91$ & $21.05 \pm 1.96$ & $19.59 \pm 2.24$ & $22.02 \pm 1.44$ \\
\hline \multirow[t]{2}{*}{ Hippocampus } & $5-\mathrm{HT}$ & $7.03 \pm 0.28$ & $6.40 \pm 0.65$ & $6.89 \pm 0.56$ & $6.57 \pm 0.63$ \\
\hline & 5-HIAA & $5.25 \pm 0.27$ & $5.78 \pm 0.47$ & $5.94 \pm 0.29$ & $6.57 \pm 0.56$ \\
\hline \multirow[t]{2}{*}{ Prefrontal Cortex } & 5 -HT & $3.63 \pm 0.26$ & $3.67 \pm 0.52$ & $4.19 \pm 0.17$ & $3.88 \pm 0.31$ \\
\hline & 5 -HIAA* & $3.53 \pm 0.25$ & $4.94 \pm 0.65$ & $3.98 \pm 0.21$ & $4.93 \pm 0.23$ \\
\hline \multirow{2}{*}{ Paraventricular Nucleus } & 5 -HT & $14.48 \pm 1.22$ & $15.06 \pm 0.96$ & $14.21 \pm 1.46$ & $15.95 \pm 1.27$ \\
\hline & 5 -HIAA $*, \Phi$ & $20.27 \pm 1.27$ & $31.70 \pm 3.30$ & $18.12 \pm 1.29$ & $13.55 \pm 1.25$ \\
\hline
\end{tabular}

Table 2.1: Summary of changes in the content of amine and monoamine neurotransmitters and their metabolites in the brains of GHSR KO and WT mice following exposure to chronic unpredictable stressors (mean concentrations were in the order of ng/g of tissue protein). NE, norepinephrine; MHPG, 3-methoxy4-hydroxyphenylglycol; DA, dopamine; HVA, homovanillic acid; DOPAC, 34-dihydroxy-phenylacetic acid; 5-HT, serotonin, 5-HIAA, 5-hydroxyindolacetic acid. * Significant main effect of treatment $(p<.05)$. ${ }^{\psi}$ Significant genotypetreatment interaction $(p<.05)$. ${ }^{\Phi}$ Significant main effect of genotype $(p<.05)$ 


\section{Chapter 3}

\section{Central Ghrelin Signalling Mediates the Metabolic Response of C57 BL6J Male Mice to Chronic Social Defeat Stress}

Z.R. Patterson, R. Khazall, H. MacKay, H. Anisman \& A. Abizaid Endocrinology, 2013

\subsection{Specific Aim 2}

Following the experiments conducted in Chapter 2, it became more evident that ghrelin is involved in the physiological response to chronic stress, and that stressinduced ghrelin secretion may contribute to the overall metabolic outcome of the stress paradigm. The previous experiments demonstrated very clearly that ghrelin signalling mediated the stress-induced changes in caloric intake, body weight and monoamine utilization in several stress-related areas of the brain. However, the potency of unpredictable stressors may in fact prevent animals from storing excess energy in the form of body mass and the subsequent development of adiposity. To circumvent this confound, a chronic social defeat stress paradigm was adopted. Chronic social defeat stress paradigms have been used to mimic human cases of chronic social stress, and the nature of this stressor has been shown to foster the development of states reminiscent of metabolic syndrome. Over the past several years many independent groups have used an array of chronic social defeat stress paradigms, all of which are slight variations of each other. The metabolic outcomes of these paradigms depend in 
large part on the parameters of the study in terms of length, severity, predictability, diet availability, presence or absence of a recovery period, strain of mouse, etc. Therefore, experiments in this chapter were aimed at characterizing the metabolic consequences of our chronic social defeat stress paradigm and describing the role of ghrelin in this process. Here, we hypothesized that stress-induced ghrelin secretion would contribute to the metabolic dysfunctions that occur as a consequence of chronic social defeat stress. To this end, we ran a series of experiments where both WT and GHSR KO mice were exposed to chronic social defeat stress and their daily metabolic activities were monitored daily (i.e. caloric intake and body weight). To further characterize ghrelin's role in mediating these metabolic changes and to identify where ghrelin signalling was important, we next exposed mice to the same social defeat stress paradigm while administering a ghrelin receptor antagonist into the left lateral ventricle. Finally, to clarify the role of age in development of stress-induced metabolic dysfunctions, we exposed both young and old WT and GHSR KO animals to the chronic social defeat stress paradigm.

\subsection{Introduction}

In mammals, the stress response involves, among other things, the activation of the sympathetic nervous system, recruitment of the hypothalamic-pituitaryadrenal (HPA) axis, changes in neurotransmitter release in several brain regions, as well as a variety of immunological changes (McEwen, 2007; Ulrich-Lai and Herman, 2009). Activation of these systems facilitates the appropriate channeling of energy resources (including increased respiration, blood pressure, and the release and use of glucose stores over fat) to promote defense, escape, and ultimately survival.

In rodents these behavioural and physiological responses are associated with the adrenal steroid corticosterone (cortisol in primates). This hormone is the final product of a cascade of events that begins with the release of $\mathrm{CRH}$ from specialized neurons in the paraventricular nucleus of the hypothalamus (PVN) into the anterior pituitary gland. This, in turn, leads to the release of ACTH into the general circulation, which ultimately stimulates the release of glucocorticoids from the adrenal cortex. This system is under the control of a negative feedback loop, whereby elevated circulating glucocorticoids travel back to the hypothalamus (Di et al., 2003, 2005; Jones et al., 1977), hippocampus (Sapolsky et al., 1984, 2000), medial prefrontal cortex (Diorio et al., 1993; Radley, 2008), and pituitary gland (Cole et al., 2000; Mahmoud et al., 1984) to inhibit further stimulation of the HPA axis via activation of glucocorticoid receptors (McEwen, 2007; Ulrich-Lai and Herman, 2009). Acutely, this response enables the behavioral and physiological adaptations necessary for the survival of an organism under stressful conditions. Repeated stressors, however, may lead to overstimulation of the HPA axis and might result in long-lasting physiological changes that ultimately lead to pathological states, including metabolic disturbances such as obesity and metabolic syndrome (McEwen, 2007; Frasure-Smith 
and Lespérance, 2005; Black, 2006). Excessive glucocorticoids, for example, have been shown to cause suppression of excitatory signals (Di et al., 2003) as well as increase inhibitory tone (Boudaba et al., 1996; Herman et al., 2002) onto $\mathrm{CRH}$ neurons in the PVN.

In addition to corticosterone, there are other hormones that are released in response to stressors, including the gut peptide ghrelin. Ghrelin is a 28amino acid hormonal peptide produced in the X/A-like cells of gastric oxyntic mucosa (Dornonville de la Cour et al., 2001). Ghrelin is best known for its ability to increase appetite and adiposity through its actions on the GH secretagogue receptor (GHSR), the only known functional ghrelin receptor (Nakazato et al., 2001; Date et al., 2006; Tschöp et al., 2000; Nogueiras, R; Lopez, M; Dieguez, 2009; Delhanty et al., 2010a; Currie et al., 2005). The GHSR is also expressed in the hypothalamus, with the highest expression found in the arcuate nucleus (Howard et al., 1996), a region critical for the regulation of food intake, metabolism, and energy homeostasis (Tschöp et al., 2000; Kirchner et al., 2009). Upon binding to the GHSR, ghrelin elicits orexigenic effects by stimulating orexigenic hypothalamic peptides including neuropeptide Y (NPY) and the Agouti-related peptide (AgRP) (Sapolsky et al., 1984; Diorio et al., 1993). Ghrelin promotes the use of carbohydrates as an energy substrate while sparing the use of body fat, ultimately tipping the energy homeostatic scales in favor of adiposity (Tschöp et al., 2000). Interestingly, plasma ghrelin levels have been shown to rise in parallel with glucocorticoids in response to both acute and chronic stressors, and ghrelin levels may remain elevated for an extended period of time after the cessation of the stressor (Asakawa et al., 2001a; Kristenssson et al., 2006; Ochi et al., 2008). Because both stress and ghrelin favour the utilization of carbohydrates as a rapid fuel source, and given that ghrelin is secreted in response to stressors, we hypothesized that ghrelin contributes to the stress-induced metabolic switch that favours carbohydrate utilization and that ultimately leads to the accumulation of fat stores. In addition, because ghrelin influences food intake and the consumption of foods that are palatable and high in caloric content, we reasoned that ghrelin promotes the intake of these types of food in response to psychosocial stressors.

\subsection{Materials \& Methods}

\subsubsection{Animals}

Male C57BL/J6 (The Jackson Laboratory, Bar Harbor, Maine), GHSR-KO mice and their wild-type (WT) littermates aged 8 - 9 weeks were used as experimental subjects. Retired breeding male CD-1 mice 13 - 15 weeks of age and weighing 40 - 50g (Charles River Farms, St Constant, Quebec, Ontario, Canada) were used as stressors. GHSR WT and KO mice were bred at Carleton University and originated from breeding pairs obtained from Regeneron Pharmaceuticals, Inc. (Tarrytown, New York). The metabolic phenotype of this strain has been previously characterized (Pfluger et al., 2008). Throughout the duration of the 
studies, mice were housed under standard laboratory conditions and received ad libitum access to standard laboratory mouse chow and tap water as well as a daily 4-hour access to a high-fat diet containing $60 \%$ caloric content from fat (TD 06414; Harlan Teklad, Indianapolis, Indiana) to measure intake of a preferred diet. All procedures were approved by the Carleton University Animal Care Committee and followed the guidelines of the Canadian Council on Animal Care.

\subsubsection{Chronic Social Defeat Stress Paradigm}

Mice were matched for body weight and caloric intake after a 21-d baseline period wherein they had ad libitum access to tap water and regular mouse chow as well as 4-hour access to a high-fat diet from 10:00AM to 2:00PM. After the baseline period, mice were assigned to either the stress or non-stress groups. Animals in the stress groups were housed in a separate room and subjected to the resident intruder paradigm to induce chronic social defeat stress (Bartolomucci and Palanza, 2005; Bartolomucci et al., 2009). In this paradigm, mice were housed with the same CD-1 mouse resident for the entire 21-day stress period. CD-1 mice were screened for aggression before the experiment and established dominance within 2-3 days of the stress period. A central divider made from transparent acrylic and wire mesh was used to prevent any physical contact between the two animals, but permitted the exchange of sensory information. Each day, at 2:00PM the divider was removed to allow interaction. The divider was replaced once the CD-1 mouse subdued the experimental mouse, or after 15 minutes had passed. After the stress period, all animals were allowed to recover for 3 weeks (returning to baseline conditions) before being sacrificed unless otherwise stated. Control animals were housed in cages without the dividers given pilot data showing that food intake and body weight of nonstressed WT and GHSR mice housed in cages with dividers does not differ from that of mice housed without dividers.

\subsubsection{Chronic Intracerebroventricular Delivery of Ghrelin Receptor Antagonist}

After a baseline period of 2 weeks, mice were assigned to groups that were matched for average body weight and caloric intake and implanted with a 31gauge stainless steel L-shaped cannula (Alzet Brain Infusion Kit no. 0004760) coupled to an osmotic mini-pump (Alzet Mini-Osmotic Pump model 2ML4; flow rate: $0.25 \mu \mathrm{l} / \mathrm{h}$ ) for 28 days via a polyethylene catheter under isofluorane/oxygen (4\%) anesthesia. The cannula was aimed at the left lateral ventricle (coordinates: anterioposterior $-0.10 \mathrm{~mm}$, mediolateral $-0.80 \mathrm{~mm}$, and dorsoventral 2.20mm; (Paxinos and Franklin, 1997). Minipumps were filled with $240 \mu$ l of either sterile saline $(0.9 \% \mathrm{NaCl})$ or a ghrelin receptor antagonist ([D-Lys $\left.{ }^{3}\right]$ GHRP-6) solution (Peptides International; 20nmol/day/mouse). The cannula was held in place secure to the skull using contact and dental cement. After 
1 week of recovery after surgery, mice were started on the 21-day experimental stress paradigm. Mice were then sacrificed by rapid decapitation, and their brains fixed by $4 \%$ paraformaldehyde. Brains were collected and sliced to confirm the accuracy of the cannula placements. Mice with missed placements were not included in the analyses.

\subsubsection{Indirect Calorimetry}

Measurements of oxygen consumption $\left(\mathrm{VO}_{2}\right)$, carbon dioxide production $\left(\mathrm{VCO}_{2}\right)$, and respiratory exchange ratio (RER) were performed using phenomaster/labmaster metabolic cages (TSE instruments, Chesterfield, Missouri). Seven days after the last stressor, mice were housed individually in the chambers for 48 hours, with regular chow and water available ad libitum. The high-fat diet was also available during the same time period because it was in their home cages. The software recorded oxygen, carbon dioxide, food intake, water intake, and locomotor activity every 30 minutes for 48 hours. Only the recordings from the latter 24 hours were used for the analyses.

\subsubsection{Tissue Processing}

Animals were killed by rapid decapitation unless otherwise stated, and plasma and tissue samples were collected and frozen at $-80^{\circ} \mathrm{C}$. To measure glucose levels, glucose strips attached to a Contour glucose meter (Bayer Corp., Pittsburgh, Pennsylvania) were dipped in trunk blood collected before being centrifuged. Brains were rapidly dissected and tissue micropunches of the mediobasal hypothalamus [1.5 to $2.0 \mathrm{~mm}$ behind bregma (Paxinos and Franklin, 1997)] were collected using hollow 16-gauge needles with a beveled tip as described byPalkovits (1973). The collection of these punches took no longer than 2 minutes after the decapitation of the animal. Finally, carcasses were frozen at $-80^{\circ} \mathrm{C}$ until they were dissected to obtain different fat pad weights. In addition, samples of the perigonadal fat were collected and stored in Trizol at $-80^{\circ} \mathrm{C}$ for histological or real time-quantitative PCR (RT- qPCR) analysis.

\subsubsection{Hormone Analysis}

After animals were sacrificed, trunk blood was collected in EDTA-coated tubes, placed on ice and centrifuged at $3000 \times \mathrm{g}$ for 15 minutes to separate plasma from red blood cells. Blood plasma was aliquoted separately for each assay to avoid multiple freeze/thaw cycles, and stored at $-80^{\circ} \mathrm{C}$ until processed. To protect the acylated ghrelin molecule, a $50 \mu \mathrm{l}$ aliquot of blood plasma was treated with $2.7 \mu \mathrm{l}$ of $1.0 \mathrm{~N}$ hydrochloric acid and $10 \mu \mathrm{l}$ of $100 \mathrm{mM}$ 4-(hydroxymercuri) benzoic acid before storage. Plasma corticosterone levels were measured in duplicates using a commercially available RIA kit (ICN Biomedicals, Inc, Aliso Viejo, California). Plasma insulin, leptin, resistin, and IL-6 were measured using a mouse adipokine milliplex kit (Mouse Serum Adipokine Immunoassay; Millipore Corp., Bedford, Massachu- setts). Plasma acylated ghrelin and adiponectin were measured using 
an ELISA kit (Millipore). All samples had a coefficient of variation $\leq 10$ and $15 \%$, respectively).

\subsubsection{Histology}

To analyze adipocyte morphology, paraffin-embedded perigonadal fat samples were sliced at $8 \mu \mathrm{m}$ and stained with hematoxylin and eosin. Adipocyte diameter was measured using ImagePro software (Media Cybernetics, Bethesda, Maryland). A total of 200 randomly selected adipocytes were used per animal.

\subsubsection{RT-qPCR}

Total RNA from brain and white adipose tissue was isolated with TriZol and precipitated with $13 \mu \mathrm{l}$ of linear acrylamide. RNA quality and concentrations were determined by absorbance at $280 \mathrm{~nm}$ and $260 \mathrm{~nm}$ with a Thermo Scientific Nanodrop 100 spectrophotometer (Thermo Scientific, Rockford, Illinois). To synthesize cDNA, $1 \mu \mathrm{l}$ oligo(dT) primer (Invitrogen, Carlsbad, California) was added to $9 \mu \mathrm{l}$ of mRNA and heated to $70^{\circ} \mathrm{C}$ for 5 minutes. To each sample, a master mix composed of $4 \mu \mathrm{l}$ of $5 \times$ first-strand buffer (Invitrogen), $2 \mu \mathrm{l}$ of $0.1 \mathrm{M}$ dithiothreitol (Invitrogen), $1 \mu \mathrm{l}$ of RNase inhibitor (Promega Corp, Madison, Wisconsin), $1 \mu \mathrm{l}$ of $10 \mathrm{mM}$ deoxynucleotide triphosphate (Invitrogen), $1 \mu \mathrm{l}$ of diethylpyrocarbonate water, and $1 \mu \mathrm{l}$ of SSR2 reverse transcriptase (Invitrogen) were added. Samples were then run on a PTC-200 Thermal Cycler (MJ Research, Watertown, Massachusetts) at $42^{\circ} \mathrm{C}$ for 1.5 hours followed by $90^{\circ} \mathrm{C}$ for 10 minutes. Samples were stored at $-20^{\circ} \mathrm{C}$. RT-qPCR was conducted on all cDNA samples to determine fold changes using the $2^{\Delta \Delta C t}$ method using primers detecting the glyceraldehyde 3-phosphate dehydrogenase gene as a control transcript (Schmittgen and Livak, 2008). Briefly, $5 \mu$ l of each cDNA sample were added to separate wells in a PCR plate. Two microliters of working primer solution, $3 \mu \mathrm{l}$ of Milli-Q water, and $10 \mu \mathrm{l}$ of iQ SYBR Green Super Mix with fluorescein (Bio-Rad Laboratories, Inc, Hercules, California) were added to each well. Samples were run in duplicate, with nontemplate controls. The plate was run on a MyIQ Single Color Real-Time PCR Detection System (Bio-Rad) for 30 seconds at $95^{\circ} \mathrm{C}$, followed by 45 cycles of the following settings: 10 seconds at $95^{\circ} \mathrm{C}$ for denaturing, 30 seconds at $55^{\circ} \mathrm{C}$ for annealing, and 20 seconds at $72^{\circ} \mathrm{C}$ for extension. The plate was then run for 1 minute at $95^{\circ} \mathrm{C}$ and 1 minute at $55^{\circ} \mathrm{C}$. The nucleotide sequences of the various primers used are provided as Supplemental Table 9.1. All primers were tested for amplification efficiency using the standard curve method, yielding efficiencies over $90 \%$. 


\subsection{Results}

\subsubsection{Chronic Social Defeat Stress Increases Plasma Ghre- lin Concentrations \& Causes Long Lasting Changes in Caloric Intake \& Body Weight}

Social stressors affect food intake, body weight, body composition and lipid metabolism in rodents and non-human primates (Bartolomucci and Pederzani, 2004; Bartolomucci et al., 2009; Chuang et al., 2010; Chuang and Perello, 2011; Tamashiro, 2007; Foster and Solomon, 2006; Schmidt, 2009). In support of these studies, Figure 3.1 shows ghrelin concentrations, caloric intake and weight gain of stressed and non-stressed mice after 3 weeks of chronic social defeat and after a 3-week recovery period. As seen in this figure, ghrelin concentrations were elevated in C57BL/J6 mice at the end of a 3-week chronic social defeat stress paradigm $\left(\mathrm{t}_{(13)}=-2.89, p<.05\right)$, and in parallel with morning corticosterone concentrations (CORT; $t_{13}=-3.89, p<.05$; see Figure 3.1). During baseline, high-fat diet consumption increased gradually, and in parallel with a gradual decrease in the intake of regular chow (see Figure 3.1), indicating that the mice developed a preference for this diet. During the stress period, however, socially defeated mice increased their total caloric intake $\left(\mathrm{F}_{(1,23)}=6.24, p<.05\right)$, their regular chow intake $\left(\mathrm{F}_{(1,23)}=9.41, p<.05\right)$, and weight gain $\left(\mathrm{F}_{(1,23)}=17.61\right.$, $p<.05)$ despite a decrease in the consumption of the calorically dense high-fat $\operatorname{diet}\left(\mathrm{F}_{(1,23)}=35.97, p<.05\right)$. More importantly, the food intake and body weight of stressed mice did not return to control levels for another week after the stress $(p<.05)$. At the end of the recovery, plasma concentrations of active ghrelin in socially defeated mice did not differ from those of non-stressed mice $\left(\mathrm{F}_{(1,23)}=0.05, p>.05\right.$; See Figure 3.1).

\subsubsection{The Effects of Chronic Social Defeat on Body Weight \& Food Intake Are GHSR-1a Dependent \& Central in Origin}

To determine the role of ghrelin in mediating the effects of stress on body weight gain and caloric intake, we assessed the impact of chronic social defeat in GHSR KO mice as well as their WT littermates. As in the previous study, chronic social defeat caused a significant increase in acylated ghrelin concentrations, coupled with an increase in body weight gain and a tendency to increased caloric intake in WT mice; these effects were attenuated in GHSR KO mice (see Figure 3.2). However, chronic social defeat increased plasma corticosterone levels similarly in both GHSR KO and WT mice, indicating that the feeding and body weight increases seen in WT mice were due to increases in ghrelin and not corticosterone (see Figure 3.2).

To determine whether these effects are mediated by the action of ghrelin at central sites, we assessed the effects of the ghrelin receptor antagonist [D-Lys ${ }^{3}$ GHRP-6 vs. vehicle administered into the left lateral ventricle of mice equipped with osmotic minipumps connected to an intracranial cannula delivery system. 
Half of the mice in each group were exposed to the chronic social defeat stress paradigm, while the other half served as non-stressed controls.

As expected, stressed animals infused with the vehicle increased both their total caloric intake and body weight $\left(\mathrm{F}_{(3,21)}=4.45, p<.05\right.$, and $\mathrm{F}_{(3,21)}=4.86$, $p<.05$, respectively; see Figure 3.3) relative to their non-sressed controls. Although stressed mice treated with the ghrelin receptor antagonist also increased their food intake and body weight, the extent of the increase was attenuated and was significantly lower than the increases observed in stressed vehicle-treated mice (see Figure 3.3).

\subsubsection{Chronic Social Defeat Favours the Use of Carbohy- drates in WT but not in GHSR KO Mice}

Chronically elevated levels of ghrelin promote the use of carbohydrates as a fuel source while conserving fat stores, metabolic changes that lead to increased adiposity (Tschöp et al., 2000). Given that chronic social defeat stress causes an increase of plasma acylated ghrelin concentrations, we hypothesized that this treatment would cause a metabolic change promoting carbohydrate use while sparing fat stores, and that this would not be evident in GHSR KO mice. To this end, we housed GHSR KO and WT mice in metabolic chambers to obtain measures of metabolic parameters using indirect calorimetry. During the baseline period, all mice showed equivalent metabolic phenotypes on all measures taken (data not shown). After the chronic social defeat paradigm, mice were allowed to recover for two weeks before being placed back into the metabolic chambers. As shown in Figure 3.3, the RER, an index of energy substrate utilization, was higher in WT stressed mice compared with WT controls, an effect that did not occur in GHSR KO mice $\left(\mathrm{F}_{(1,31)}=3.95, p<.05\right)$. Given these data, we suspected that stressed GHSR KO mice would show depletion of fat stores. An analysis of fat pad weights after death indeed revealed that GHSR KO mice had the lowest total fat pad weights, although only the differences in intrascapular brown fat pad and subcutaneous fat pad weights attained statistical significance $\left(\mathrm{F}_{(3,38)}=7.37 \& 4.18\right.$ respectively, $p<.05$; see Figure 3.4$)$.

\subsubsection{The Divergent Metabolic Consequences of Chronic Social Defeat Stress Are Exacerbated in Aged WT \& GHSR KO Mice}

The expression of GHSR in adipose tissues is very low in young mice, but increases as the mice age, and this increase has been associated with increased adiposity and altered metabolism (Lin et al., 2011). Because of this, we suspected that chronic social defeat would have a greater impact on metabolism in mice that were older than those we used in the previous study. We therefore exposed 6- to 7-month-old GHSR KO and WT mice to the chronic social defeat stress and allowed them to recover for an additional 3 weeks before being sacri- 
ficed.

Results from this study showed that chronic social defeat caused an increase in visceral (perigonadal/inguinal) fat pad and intrascapular brown fat pad weights in WT, but not in GHSR KO mice, relative to their non-stressed controls $\left(\mathrm{F}_{(3,36)}=4.69 \& 4.57\right.$ respectively; $p<.05$; see Figure 3.5). Histological analysis from visceral fat samples showed that WT stressed mice had significantly larger adipocytes than WT non-stressed mice, with no differences existing between stressed and non-stressed GHSR KO mice $\left(\mathrm{F}_{(3,33)}=8.60, p<\right.$ .05 ; see Figure 3.5). In concert with these measures of adiposity, WT stressed mice also showed markers of obesity such as hyperinsulinemia $\left(\mathrm{F}_{(3,30)}=8.57\right.$, $p<.05)$, elevated basal corticosterone levels $\left(\mathrm{F}_{(3,33)}=3.44, p<.05\right)$ and an increase of IL- $6\left(\mathrm{~F}_{(3,33)}=5.80, p<.05\right.$; see Figure 3.6) relative to their nonstressed controls. Furthermore, WT mice tended to have higher levels of blood glucose and leptin relative to $\operatorname{GHSR} \operatorname{KOs}\left(\mathrm{F}_{(3,31)}=3.33 \& \mathrm{~F}_{(3,25)}=2.981\right.$ respectively, $p<.10$; See Figure 3.6).

Gene expression analysis of white adipose tissues using RT-qPCR showed that visceral white adipose tissue obtained from stressed GHSR KO mice had decreased expression of fatty acid synthase, an enzyme important for the anabolic processes associated with storing fat as well as an enzyme that is decreased after fasting and ghrelin treatment $\left(\mathrm{F}_{(3,28)}=3.38, p<.05\right.$, Appendix A - Figure 8.1). Other transcripts associated with lipid handlings such as lipoprotein lipase, acetyl CoA carboxylase, and the NPY Y2 receptor (Y2-R) were not difference between the strains of mice and were unaltered after chronic social defeat (Supplemental Figure 3.1). The metabolic profile of WT stressed mice was positively correlated with the expression of hypothalamic peptides that are elevated during negative energy balance. For instance, WT stressed mice had increased hypothalamic NPY $\left(\mathrm{F}_{(3,28)}=7.21, p<.05\right)$; and AgRP gene expression $\left(\mathrm{F}_{(3,28)}\right.$ $=4.13, p<.05$; see Figure 3.6).

\subsection{Discussion}

Physiological responses to stress include a number of endocrine changes that affect both the periphery and the brain. In the short term these changes allow for an organism to generate sufficient energy to meet the challenges presented by the stressors. Over prolonged periods of time, however, these protective mechanisms may themselves become pathological (McEwen, 2007). Here we present data suggesting that ghrelin, a metabolic hormone that is released after stress to protect the periphery and the brain, can ultimately lead to metabolic changes that promote obesity in the face of chronic exposure to stress. Furthermore, these effects may be mediated, in part, by the action of ghrelin in the brain.

The protective effects of ghrelin have been highlighted in a number of studies using both acute and chronic stress paradigms. Indeed, ghrelin is secreted in response to acute stressors (Kristenssson et al., 2006; Spencer et al., 2012), and exogenous ghrelin treatment evokes the release of ACTH and prolactin from the pituitary (Schmid et al., 2005; Tassone et al., 2003). Chronic stressors also 
increase ghrelin concentrations that persist for some time beyond the duration of the stress protocol (Ochi et al., 2008; Lutter and Sakata, 2008). Peripherally, ghrelin seems to protect the stomach against ulcerations that occur after chronic stress (Ochi et al., 2008). However, ghrelin serves a number of functions other than gastro-protection after stress. For instance, ghrelin attenuates the display of anxiety and depressive-like behavior in rodents (Lutter and Sakata, 2008; Alvarez-Crespo et al., 2012; Kanehisa et al., 2006). In contrast, GHSR KO mice display more of these behaviors after 10 days of daily social defeat (Lutter and Sakata, 2008). In all, these data suggest that ghrelin signalling during stress is important to counter the effects of chronic stressors, and the lack of ghrelin signaling may lead to psychopathological conditions such as depression (Lutter and Sakata, 2008).

The protective effects of ghrelin could come from promoting energy replenishment, where ghrelin promotes an increase of appetite to offset the energetic costs of dealing with a stressful event. This effect has been reported previously (Lutter and Sakata, 2008; Kanoski et al., 2013) and is confirmed in our current studies in which exposure to chronic social defeat in mice produced increased plasma acylated ghrelin concentrations, and this occurred in tandem with increased caloric intake and body weight. Unexpectedly, the stress-induced increase in caloric intake did not come from an increase in the consumption of the calorically dense diet that the animals clearly preferred before the stress paradigm began. During the baseline, mice in our studies clearly increased their intake of the high-fat diet when it became available, while decreasing their intake of regular chow. When stressed, however, mice clearly decreased their intake of the high-fat diet, but increased their intake of the regular chow, an effect that was slowly reversed during the recovery period. Although we expected that stressed mice would eat more of this preferred diet in anticipation of the stressor, they ate less. In contrast, others have shown that mice increase the intake of high-fat pellets, and show stronger conditioned place preferences to these pellets after chronic social defeat stress, whereas GHSR KO mice do not show these preferences (Chuang and Perello, 2011). One possibility for the discrepency between our results and those of others (Chuang and Perello, 2011) may have to do with the timing and the context in which the preferred diet is presented. Thus, although mice may engage in increased reward-based eating and develop conditioned place preferences to palatable foods when tested after being stressed (Chuang and Perello, 2011), this may not be the case if tested while they are being stressed, as is the case in our studies, where they clearly show a decrease in their preference for the high-fat diet. Moreover, it is possible that in our studies, stressed mice associated the presence of the diet with the oncoming stressors, ultimately reducing the intake of the high-fat diet. Hours after the stress bout has occurred, but when the high fat is no longer available, stress-induced ghrelin secretion produced an increase in chow intake that was significant enough to increase total caloric intake.

A second protective mechanism would be one in which ghrelin favors the utilization of carbohydrates to meet the rapid energetic challenges posed by a chronic stressor. In the present studies we show that WT stressed mice favor 
the utilization of carbohydrates as a fuel source, while sparing fat utilization as reflected in a higher RER compared with non-stressed mice. This difference, however, was absent in GHSR KO mice. Interestingly, stressed GHSR KO mice tended to have the lowest amounts of fat pads overall, and this was particularly evident in the brown and subcutaneous adipose depots. Thus, it appears that GHSR KO stressed mice use more of these depots as a fuel source relative to WT stressed mice, or that they are less capable of storing fat while stressed. The change in body composition seen in GHSR KO stressed animals is likely a product of their energy substrate utilization and not due to a difference in food intake. For instance, young WT stressed animals showed an increase in caloric intake relative to their non-stressed controls, but no differences in body composition. In contrast, GHSR KO stressed animals do not show changes in caloric intake compared with their non-stressed controls, but do show a reduction in fat deposition, supporting the notion that they continue to use fat depots as fuel. If differences in food intake were to dictate the overall accumulation of adipose tissue, one would expect stressed WT mice to show increased adiposity whereas GHSR KO mice would not show a drop in adipose tissue because they continued to eat the same as in the baseline.

Stress-induced increases in caloric intake, along with decreased use of fat as fuel, would be expected to result in increased adiposity over time. Young WT mice in our studies, however, did not show long-term increases in fat accumulation after the stress paradigm, suggesting that, despite stressor-induced increases in food intake, body weight, and RER, they ultimately recovered and did not accumulate more body fat than non-stressed animals. In contrast, older WT mice showed clear signs of metabolic imbalance following the same chronic social defeat stress paradigm. Among these, mice showed increased accumulation of visceral white adipose tissue, as well as increased intrascapular brown fat mass. In contrast, stressed GHSR KO mice had the lowest accumulation of adiposity in these same regions. Older stressed WT mice showed adipocytes that were significantly larger in size than those of non-stressed WT mice, a difference not seen in GHSR KO mice. Although these are crude measures of adiposity, and a more accurate measure of body composition is required, they do correlate well with other measures of adiposity, such as higher plasma concentrations of leptin, insulin, glucose, and IL-6, a difference that was absent in GHSR KO animals. Finally, the hypothalamus of WT stressed mice showed increased NPY and AgRP mRNA expression, whereas hypothalamic gene expression among GHSR KO mice remained equivalent to that of their non-stressed controls. It is important to note that these plasma analyses were obtained from samples collected 2 weeks after the last stressor. Therefore, not only are older WT mice less able to maintain metabolic homeostasis during chronic stress paradigms, but these alterations are long lasting. More importantly, old GHSR KO mice did not show these alterations, suggesting that ghrelin is responsible for these alterations.

Ghrelin's effect on food intake and metabolism are mediated by the action of ghrelin on the GHSR-1a both in the brain and in white adipose tissue (Tschöp et al., 2000; Lin et al., 2011). Effects of ghrelin directly on adipose tissue, how- 
ever, are more pronounced as animals (including humans) age. It has been suggested that increases in adiposity in older animals is a function of increased adipose tissue GHSR expression in older animals (Lin et al., 2011). Our data suggest that the effects of chronic social defeat on energy balance are mediated, in part, by central ghrelin signalling. Indeed, GHSR KO mice, and mice treated chronically with intracerebroventricularly with [D-Lys ${ }^{3}$ ]-GHRP-6 during the stress period, showed attenuated feeding and weight gain responses, while maintaining elevated concentrations of corticosterone. There are several central sites at which ghrelin could act to increase food intake and body weight during the chronic social defeat, because ghrelin receptors are located in a number of brain sites known to be part of both regulation of energy balance and stress including the PVN, hippocampus, ventral tegmental area (VTA), the edingerwestphal nucleus, the nucleus of the solitary tract (NTS) in the brain stem, and the amygdala (Alvarez-Crespo et al., 2012; Kanoski et al., 2013; Zigman and Jones, 2006; Faulconbridge et al., 2003; Currie et al., 2012; Abizaid and Liu, 2006); however, the relative contributions of each to stress-induced feeding remains to be determined. For instance, ghrelin could be acting directly onto the PVN to alter substrate utilization and favor carbohydrate metabolism (Currie et al., 2012). Ghrelin may act on the hippocampus to protect against damage from chronic glucocorticoid exposure after chronic social defeat while promoting the effectiveness of cues that predict availability of foods to elicit feeding (Kanoski et al., 2013; Razzoli and Domenici, 2011). In the VTA, ghrelin may increase the motivation to eat palatable foods after chronic social defeat particularly through the stimulation of VTA dopamine neurons (Chuang and Perello, 2011; Abizaid and Liu, 2006; Perello et al., 2010; Skibicka et al., 2011; King et al., 2011). In the edinger-westphal nucleus, ghrelin could increase urocortin release, which, in turn, could modulate feeding and lipid metabolism after chronic stress (Spencer et al., 2012; Chao et al., 2012). Finally, stress-induced ghrelin secretion may alter sympathetic responses and glucose-regulatory processes by actions on GHSRs located in the nucleus of the solitary tract (Scott et al., 2012).

There are some limitations in our study that must be considered. For instance, the ghrelin receptor antagonist used in this study is not entirely selective to GHSR (Depoortere et al., 2006; Patel et al., 2012). It has been shown that [D$\mathrm{Lys}^{3}$ ]-GHRP-6 is also capable of acting as an agonist to the $5-\mathrm{HT}_{2 B}$ receptor to influence the contraction of fundic strips (Depoortere et al., 2006). Furthermore, [D-Lys $\left.{ }^{3}\right]$-GHRP-6 is also capable of regulating chemokine signalling through interactions with CXCR4 in immune cells (Patel et al., 2012). Whether similar effects occur centrally has not yet been determined, and given that antagonisttreated mice show a profile similar to that of GHSR KO mice when exposed to the chronic social defeat, we tend to attribute these effects to the influence of [D-Lys $\left.{ }^{3}\right]$-GHRP-6 on the GHSR and not to serotonin or chemokine receptors. However, future studies need to be conducted to determine this as better GHSR antagonists become more readily available.

It is also important to note that the endocrine responses seen after the chronic stress period were not as marked as expected, particularly in mice killed 
24 hours after their last stressor. For instance, regardless of their genotype, stressed mice showed significantly higher corticosterone levels, but theses were only about $10 \%$ higher than controls. In addition, GHSR KO mice did not have increased ghrelin concentrations 24 hours after their last stressor, whereas WT mice did. This is in contrast with studies using chronic social defeat paradigm similar (but not the same) to the one used here, or using chronic unpredictable stressors, wherein increased plasma ghrelin concentrations were seen in GHSR KO mice (Chuang and Perello, 2011; Patterson and Ducharme, 2010). Given that in our studies stressed mice are exposed to the same aggressive male for 21 days, the lower than expected corticosterone levels seen in stressed mice and/or lower ghrelin concentrations observed in stressed GHSR KO mice, may reflect habituation to the familiar aggressive males (Anisman and Matheson, 2005). This also would explain differences in corticosterone seen after the social defeat paradigm that we used here and those seen by our group when using a chronic unpredictable stressor paradigm (See Chapter 2). In spite of this, basal corticosterone levels from mice in these studies were elevated and maintained for at least 2 weeks after the end of the stress, suggesting that our stress protocol was not only sufficient to elicit the activation of the HPA axis, but that this system continued to be excited for several days after the stress protocol was terminated.

Overall these data suggest that stress produces an increase of ghrelin that increases food intake and promotes the utilization of carbohydrates over fat, in order to meet the energetic challenge posed by the stressor. This allostatic change is helpful in the short term, but in the long term it has an impact on metabolism, particularly in older mice whose ability to maintain energy balance is compromised (see Figure 3.7). Stressed mice with mutations to the GHSR gene do not show these allostatic changes. Instead, GHSR KO mice meet the energetic demands of the chronic stressor by increasing the use of fat as a fuel source. This physiological change, however, may be insufficient to protect these mice against the effects of chronic stress and may make them more vulnerable to stress-induced depression (Lutter and Sakata, 2008). 


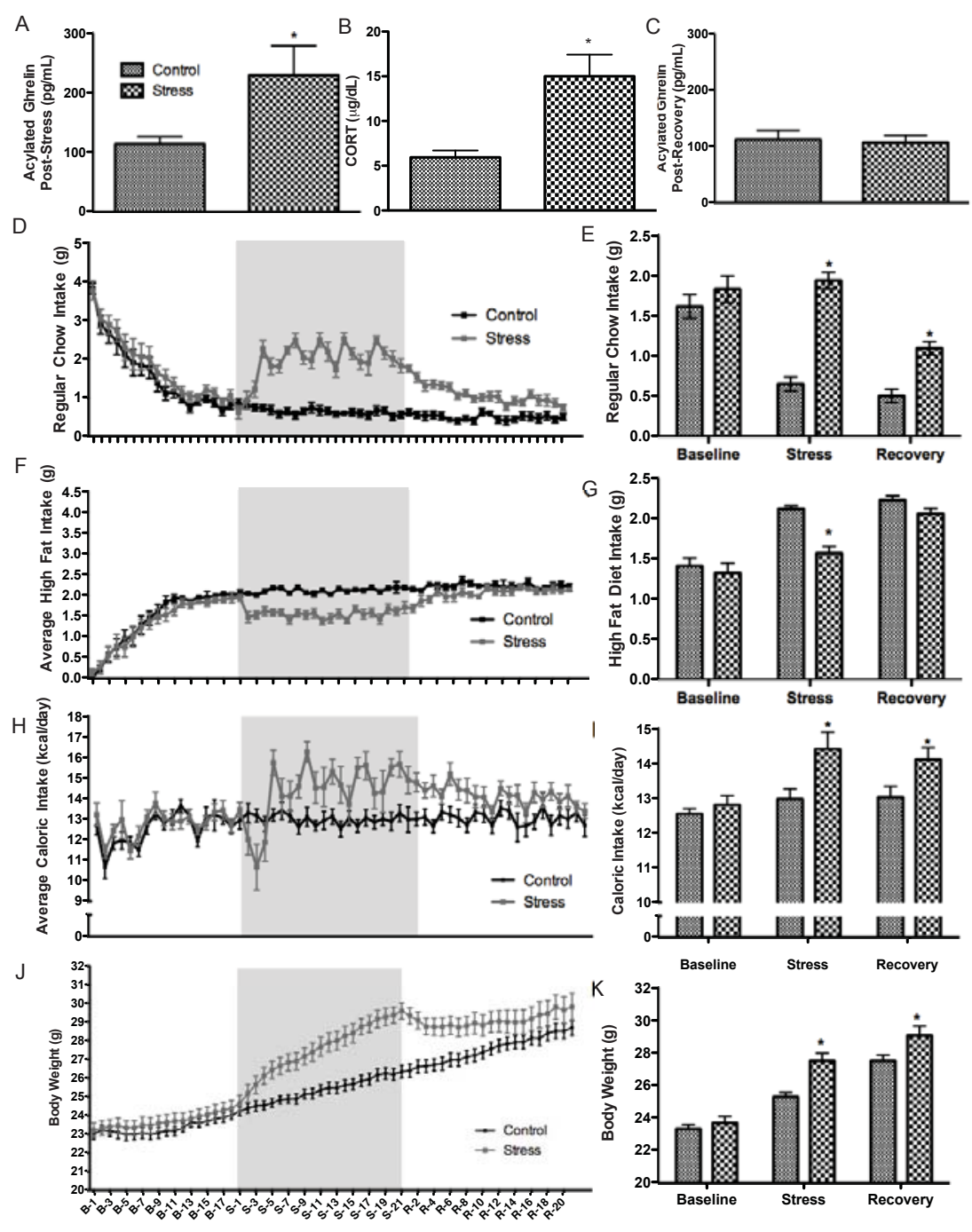

Figure 3.1: Acylated ghrelin levels 24 hours after stress (A), plasma corticosterone levels 24 hours after stress (B), plasma acylated ghrelin 21 days after their last social defeat episode $(\mathrm{C})$, daily regular chow consumption $(\mathrm{D})$, average regular chow consumption (E), daily high-fat diet intake $(\mathrm{F})$, average high-fat diet intake $(\mathrm{G})$, daily caloric intake $(\mathrm{H})$, average caloric intake $(\mathrm{I})$, daily body weights $(\mathrm{J})$, and average body weights $(\mathrm{K})$ for stress \& non-stressed controls during the baseline period, stress period \& recovery period. All values are express as mean \pm SEM. Baseline period is denoted as B1-B21; stress period is denoted as S1-S21 and is illustrated with shaded box, and recovery period is denoted as R1-R21. ${ }^{*} p<.05$ relative to non-stressed controls 

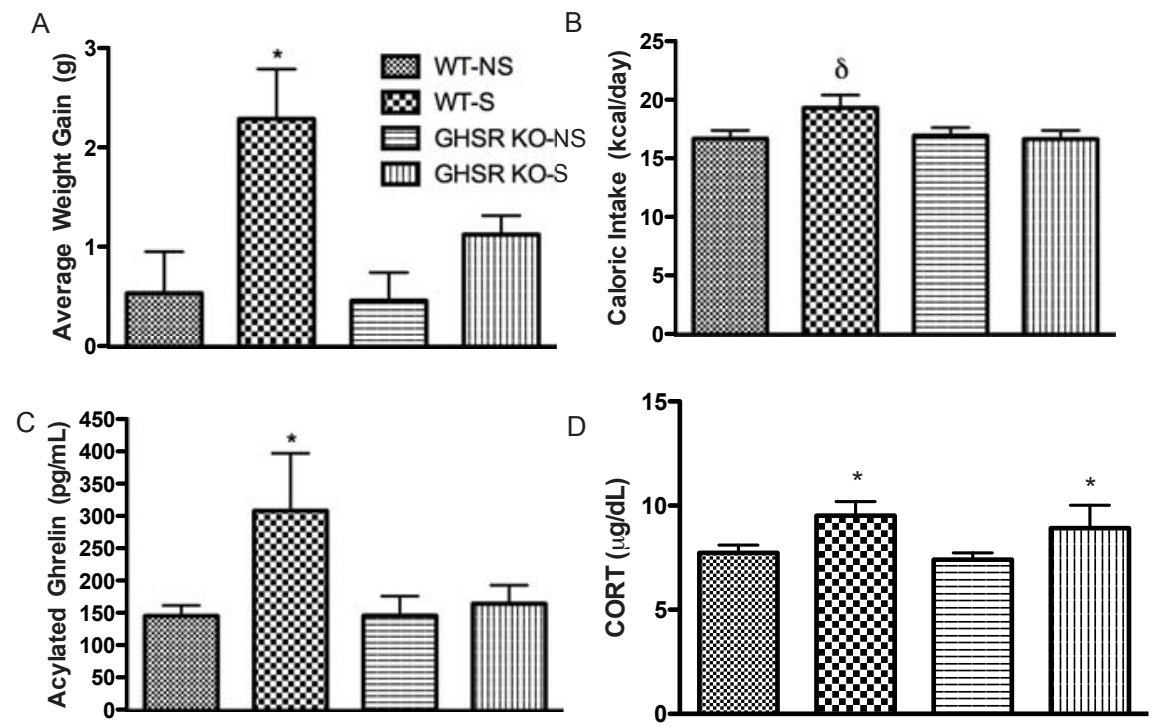

Figure 3.2: Weight gain (A) \& caloric intake (B) during the chronic social defeat stress paradigm. Acylated ghrelin levels (C) \& corticosterone (CORT; D) from trunk blood collected at time of sacrifice. All values are expressed as mean \pm SEM. ${ }^{*} p<.05 ;{ }^{\delta} p<.10$ relative to non-stressed controls. 

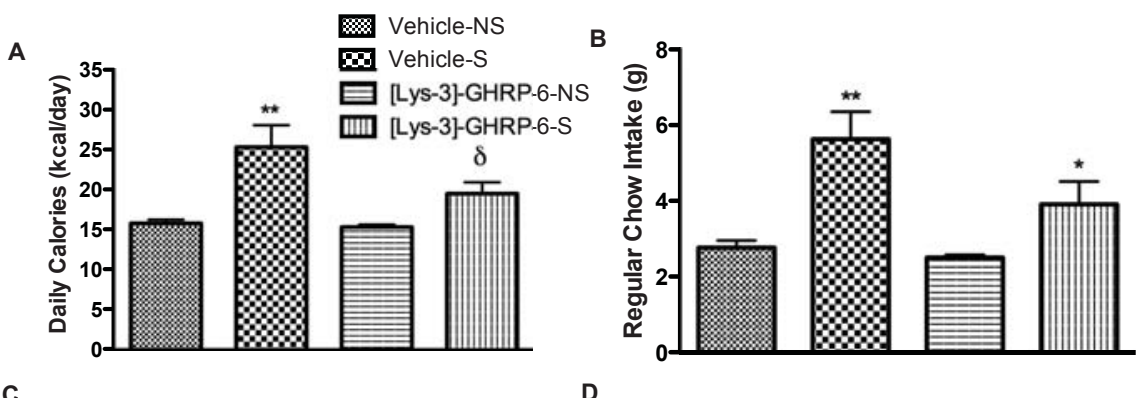

C

D
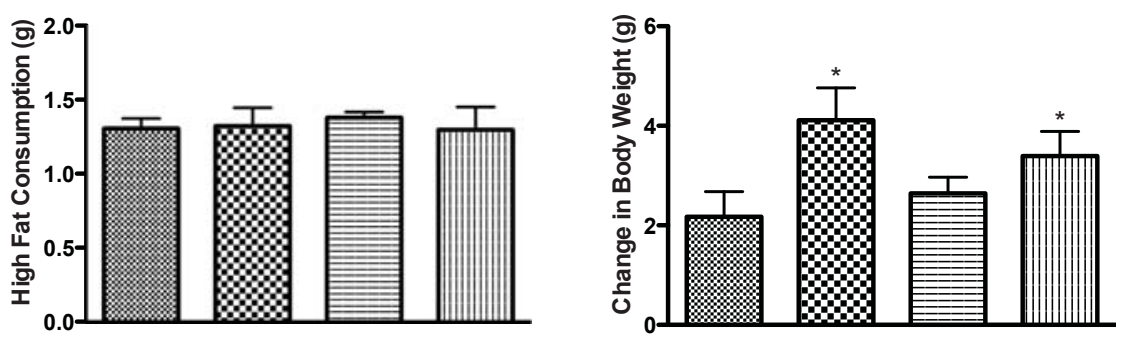

Figure 3.3: Metabolic profile of animals receiving central infusions of either vehicle (0.9\% saline) or a ghrelin receptor angtagonist ([D-Lys $\left.\left.{ }^{3}\right]-G H R P-6\right)$ in response to chronic social defeat. Average daily caloric consumption (A), chow consumption (B), high-fat diet consumption (C) and average change in body weight during the stress period (D). All values are reported as mean $\pm \mathrm{SEM}$. ${ }^{* *} p<.01 ;{ }^{*} p<.05 ;{ }^{\delta} p<.10$ vs non-stressed controls. 

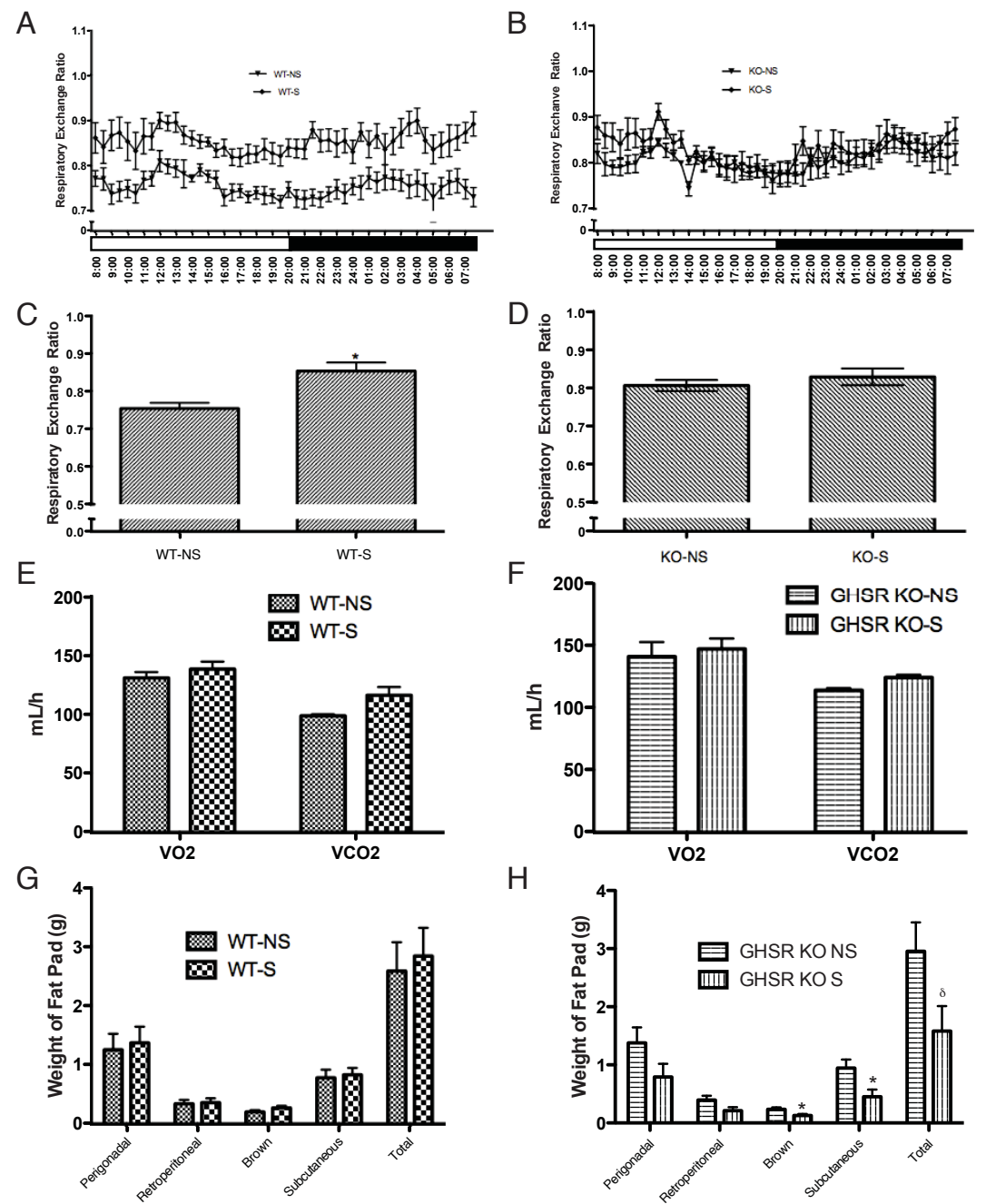

Figure 3.4: RER measured via indirect calorimetry during the recovery period vs time of day for WTs (A) and GHSR KOs (B), average daily RER for WTs (C) and GHSR KOs (D), average oxygen consumption and carbon dioxide production for WTs (E) and GHSR KO (F) and fat pad weights for WTs $(\mathrm{G})$ and GHSR KOs $(\mathrm{H})$. All values are reported as mean \pm SEM. ${ }^{*} p<.05 ;{ }^{\delta} p<.10$ vs non-stressed controls. 

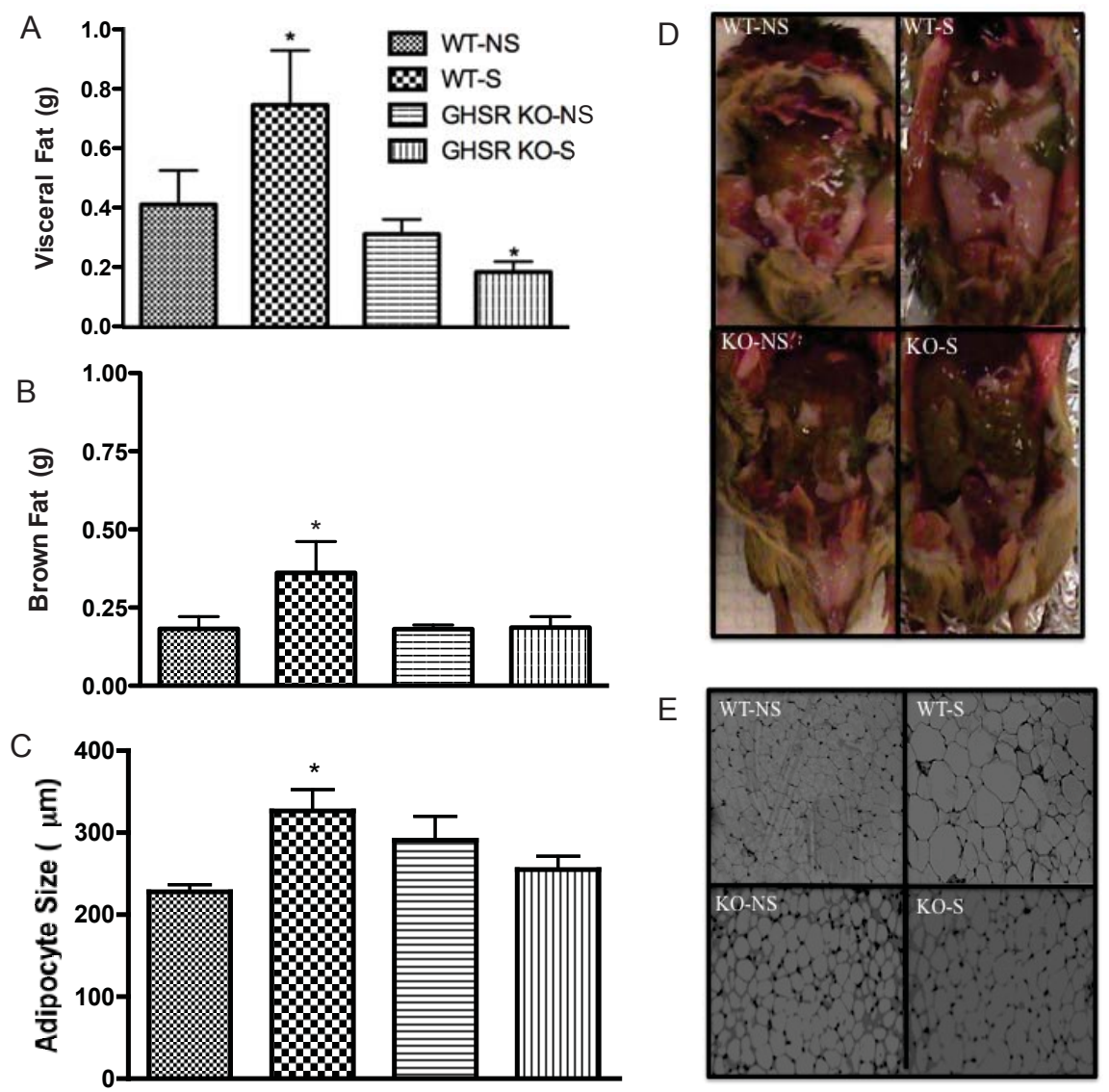

Figure 3.5: Average weight of visceral fat (A), average weight of intrascapular brown fat (B), and the average diameter of visceral adipocytes (C). Illustration of visceral adipose tissue (D) and average diameter of visceral adipocytes (E). ${ }^{*} p<.05$ vs non-stressed controls 


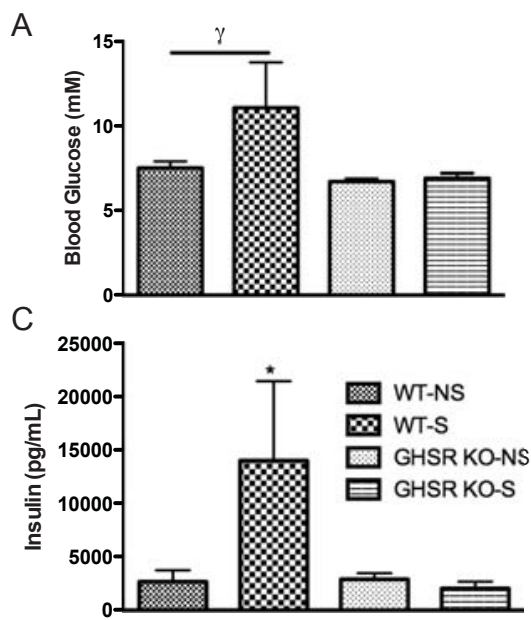

$E$

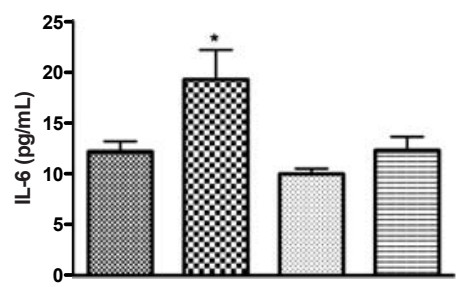

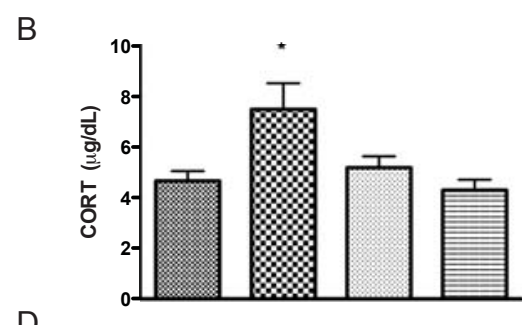

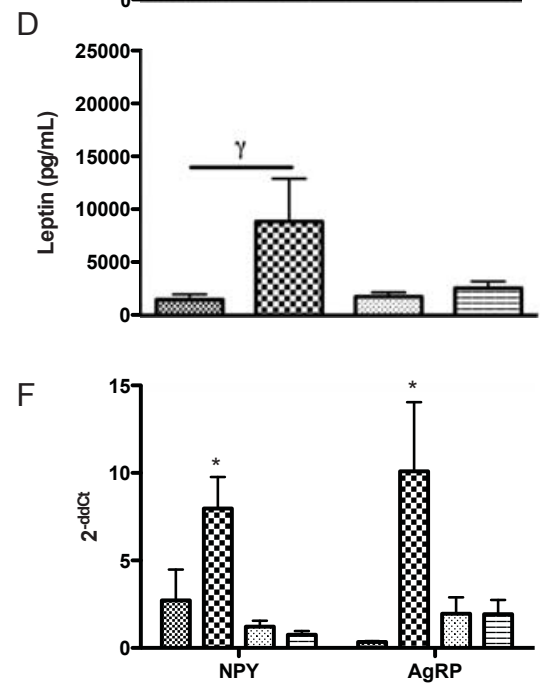

B

F

Figure 3.6: Hormonal profile of trunk blood collected at the time of sacrifice. Mean concentration of plasma blood glucose (A), corticosterone (B), insulin (C), leptin (D) and IL-6 (E). All values are express as mean \pm SEM. mRNA expression of NPY \& AgRP in the mediobasal hypothalamus (F). All values are expressed as mean $\pm \mathrm{SEM}$, relative to GAPDH expression. ${ }^{*} p<.05$ relative to non-stressed controls; ${ }^{\gamma} p<.10$ vs GHSR KOs. 


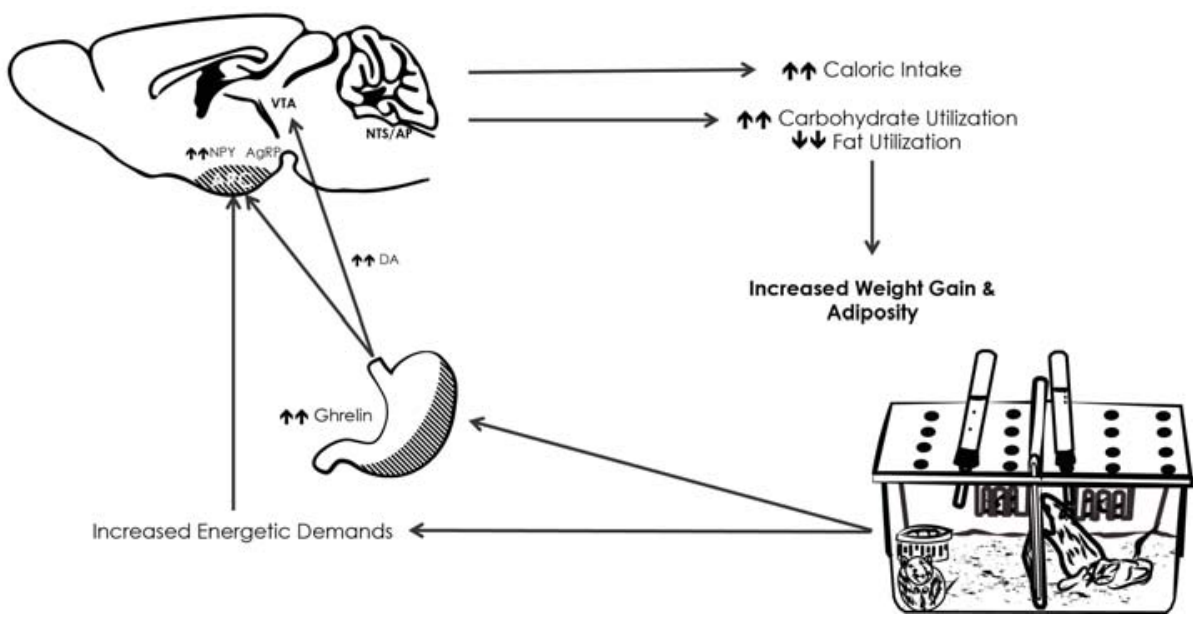

Figure 3.7: Proposed explanation of how ghrelin mediates the metabolic \& behavioral adaptations necessary to deal with stress. Chronic stress produces an energetic demand that is met by increased caloric intake and by switching metabolism to favor the utilization of carbohydrates over fat as a source of fuel. This effect is mediated by ghrelin, and lack of GHSR prevents this metabolic switch from occurring. In the long run chronically increased levels of ghrelin may lead to obesity and metabolic disorders. 


\title{
Chapter 4
}

\section{Interruption of Ghrelin Signalling in the PVN Increases High Fat Diet Intake \& Body Weight in Male C57BL6J Mice}

\author{
Z.R. Patterson, T.J. Parno, A. Isaacs \&3 A. Abizaid
}

Frontiers in Neuroendocrine Science, 2013

doi: $10.3389 /$ fnins.2013.00167

\subsection{Specific Aim 3}

As previously described, GHSR-1a is expressed in several stress and feeding related areas of the brain. Given the role of central ghrelin signalling in mediating the metabolic switch that promotes the intake and utilization of carbohydrates and subsequent increases in adiposity, we next wanted to examine the role of ghrelin signalling in specific hypothalamic nuclei. The PVN is a structure known to express GHSR-1a and is sensitive to exogenous ghrelin administration (Currie et al., 2005, 2012; Cabral et al., 2012). Ghrelin administration into the PVN elicits an increase in respiratory quotient suggesting that this region may mediate the ghrelin-induced promotion of carbohydrate utilization. Moreover, the PVN has a well defined role in the initiation and termination of the physiological responses generated in the face of stress, and ghrelin has been shown to indirectly mediate the activity of CRH neurons within the PVN (Cabral et al., 2012). For this reason, we hypothesized that ghrelin signalling in the PVN would mediate the metabolic switch that occurs following chronic social defeat stress, 
and blockade of ghrelin signalling in the PVN would disrupt the preference for carbohydrate rich diets in stressed animals. To this end, we exposed mice to the chronic social defeat stress paradigm while simultaneously delivering a ghrelin receptor antagonist selectively in the PVN.

\subsection{Introduction}

In mammals, environmental stressors result in the activation of a number of homeostatic and allostatic physiological mechanisms that enable the organism to deal with the impending threat (McEwen, 2007). One of these mechanisms involves the activation of the hypothalamic pituitary adrenal (HPA) axis, a major neuroendocrine system that controls the response to stress. Thus, in the face of a stressor, parvocellular cells in the paraventricular nucleus of the hypothalamus $(\mathrm{PVN})$ secrete corticotropin release hormone $(\mathrm{CRH})$ onto the portal system in the median eminence and onto the anterior pituitary. Here CRH acts on corticotropes to elicit the secretion of adrenal corticotropin releasing hormone (ACTH) into the blood stream (Smith and Vale, 2006). Increased circulating levels of ACTH stimulate cells in the adrenal cortex to release glucocorticoids (Stratakis and Chrousos, 1995; Smith and Vale, 2006). Increased levels of glucocorticoids are important for a number of reasons that include the release of glucose and glycogen stores from the liver and muscle, and the release of insulin from the pancreas facilitating the generation of energy required to meet the energetic demands posed by the stressor (Black, 2006). Glucocorticoids then provide a negative feedback signal to the hypothalamus and hippocampus to terminate any further activation of the HPA axis (McEwen, 2007).

Recently, the orexigenic hormone ghrelin has emerged as another hormone that is secreted in response to stressors and one that may produce some of the metabolic changes required to deal with the energetic demands posed by the stressors. In addition to increasing food intake and growth hormone secretion, ghrelin promotes the utilization of carbohydrates as a fuel source, while preventing the use of lipids, resulting in increases in body weight due to increases in body fat (Kojima et al., 1999; Tschöp et al., 2000). Moreover, these effects are produced by ghrelin acting centrally (Tschöp et al., 2000; Nakazato et al., 2001). Under normal conditions, ghrelin is secreted in circadian patterns, or in anticipation of scheduled meals (Cummings et al., 2001; Drazen et al., 2006; Blum et al., 2009). Nevertheless, ghrelin is also secreted in response to acute stressors, and circulating ghrelin levels are higher in chronically stressed animals (Lutter and Sakata, 2008). Thus, animals that are exposed to repeated social stressors often show increased caloric intake, body weight and adiposity in tandem with increased ghrelin concentrations (Lutter and Sakata, 2008; Bartolomucci et al., 2009; Kumar et al., 2013). Work from Chapter 3 demonstrates that ghrelin mediates these effects through its action on brain sites that contain GHSR-1a. These data support the notion that stress generates a metabolic change, and that ghrelin is released as part of the physiological mechanisms that are generated to meet this challenge. Interestingly, mice with mutations 
to the only known ghrelin receptor, the growth hormone secretagogue receptor (GHSR), are more susceptible to develop depressive like behaviors after chronic social stress compared to their wild type (WT) littermates (Lutter and Sakata, 2008).

While the work from Chapter 3 clearly demonstrates that stress-induced ghrelin secretion affects food intake and energy balance through central actions, not much is known about the relative contribution of different ghrelin-sensitive brain regions, many of which are implicated in the behavioural and metabolic responses to stress. Among these regions, the PVN stands out given its role in the regulation of the HPA axis as well as the modulation of sympathetic responses, and its connections with peripheral tissues such as the liver, pancreas and adipose tissues (Hill, 2012). Ghrelin receptors are found within the PVN although it appears that they do no co-localize with CRH producing neurons (Guan et al., 1997; Zigman and Jones, 2006; Cabral et al., 2012). Nevertheless, ghrelin acting on other cells in the PVN could be responsible for some of the behavioural and metabolic alterations that are produced by exposure to chronic social stress (Cabral et al., 2012). To examine this possibility, we exposed mice to chronic social defeat stress for a period of twenty one days while being infused with a ghrelin receptor antagonist ([D-Lys-3-GHRP-6) or vehicle (0.9\% saline) and compared their metabolic phenotypes with non-stressed controls receiving the same drug or vehicle infusions.

\subsection{Materials \& Methods}

\subsubsection{Animals}

Male C57BL6J mice weighing 20-22 grams were obtained from Charles River farms, St. Constant, Quebec as experimental subjects. Mice $(\mathrm{N}=48)$ were housed under standard laboratory conditions with ad libitum access to mouse chow $(3.3 \mathrm{kcal} / \mathrm{g}$, with $70 \%$ of calories derived from carbohydrates) and tap water in addition to daily 4-hour high fat diet containing $60 \%$ caloric content from fat (TD 06414, Harlan). The calculated metabolized energy of the high fat diet was $5.1 \mathrm{kcal} / \mathrm{g}$ with $60 \%$ calories from fat. Male CD-1 retired breeder mice weighing 40-50 grams, also obtained from Charles River Farms, were used as aggressors in the chronic social defeat stress paradigm. Food intake (both standard laboratory chow and high-fat diet) and body weight were weighed and recorded daily by the experimenter at 9:00AM. The high-fat diet was available from 10:00AM 2:00PM daily. Following the baseline period, mice were separated into 4 experimental groups as follows: vehicle stressed $(\mathrm{n}=12)$, vehicle non-stressed $(\mathrm{n}=$ 12), [D-Lys-3]-GHRP-6 non-stressed $(\mathrm{n}=12)$ and [D-Lys-3]-GHRP-6 stressed $(\mathrm{n}=12)$. Due to attrition of animals throughout the 28-day cannula-minipump infusion process, as well as misplaced cannulae, the final group numbers used for data analysis were as follows: vehicle stressed $(\mathrm{n}=6)$, vehicle non-stressed ( $\mathrm{n}$ $=10)$, [D-Lys-3]-GHRP-6 non-stressed $(\mathrm{n}=4)$ and [D-Lys-3-GHRP-6 stressed

$(\mathrm{n}=6)$. All procedures documented were approved by the Carleton University 
Animal Care Committee and the guidelines of the Canadian Council on Animal Care were followed.

\subsubsection{Stereotaxic Surgery - Chronic Delivery of Ghrelin Receptor Antagonist Into the Paraventricular Nu- cleus of the Hypothalamus}

Prior to surgery, we conducted a two-week baseline where measures of food intake (both standard laboratory chow and high fat diet) and body weight were recorded daily. After baseline, mice were anesthetized using isofluorane mixed with oxygen $(4 \%)$ and implanted with an intracranial cannula connected to an osmotic minipump delivering either saline or the ghrelin receptor antagonist [D-Lys-3]-GHRP-6. To do this, the mouse's head was shaved and secured onto a mouse stereotaxic apparatus while anesthetized (Kopf Instruments, Tujuanga, CA). The scalp was cleaned with surgiprep and privodine to provide an aseptic canvas. Tear gel was applied to prevent dehydration of the eyes. A midline incision was made and the skin was retracted for a clear visualization of bregma. A 28 gauge stainless steel unilateral cannula was coupled with an osmotic mini-pump (Alzet Mini-Osmotic Pump - Model 1004; flow: 0.11 $\mu / \mathrm{hr}$ for 28 days) using a polyethylene catheter and was implanted into the paraventricular nucleus of the hypothalamus (PVN). Sterotactic coordinates of the cannula, relative to bregma, were AP $-0.94 \mathrm{~mm}$, ML $-1.75 \mathrm{~mm}$ and DV $-4.83 \mathrm{~mm}$ (Paxinos and Franklin, 1997). Mini-pumps were filled with $100 \mu \mathrm{L}$ of the ghrelin receptor antagonist ([D-Lys-3]-GHRP-6) solution (Peptides International; $20 \mathrm{nmol} /$ day $/$ mouse $)$ or vehicle $(0.9 \%$ saline $)$. The implant was secured with contact and dental cement. When the cement was dry, the dorsal portion of the skin was separated from the muscle using blunt dissection to implant the mini-pump subcutaneously. The incision was closed using silk surgical sutures. Polysporin and Lidocaine were applied topically to the surgical site to prevent bacterial infection and pain, respectively. Mice were also injected with a low dose of meloxicam (Metacam, $0.1 \mathrm{~mL}$ of $5 \mathrm{mg} / \mathrm{mL}$ ) to provide postoperative analgesia. Following surgery, mice were moved to a recovery area in a clean cage with a heating pad. Upon wakening, mice were monitored closely for optimal recovery for a total of seven days following surgery.

\subsubsection{Chronic Social Defeat Stress Paradigm}

Following the one week recovery period after surgery, half of the saline and half of the ghrelin receptor antagonist infused mice were taken from their room and transported to another room where each of them was housed in a cage inhabited by a much larger sexually experienced male CD-1 mouse for the next 21 days (as discussed in Chapter 3). The experimental mice were protected from the aggressive CD-1 mice by an acrylic divider and wire mesh, yet olfactory, visual and auditory contact was maintained with the resident for the entire 21 day stress period. Each day the divider was removed and the animals were permitted to interact until the experimental mouse was subdued or until 15 minutes had 
passed. Measurements of regular chow intake, high fat diet intake, and body weight were recorded each day during the stress period.

\subsubsection{Blood Plasma Collection}

At the end of the 21 days of chronic social defeat stress, mice in the stress group were sacrificed by rapid decapitation, the morning following the last social interaction with the dominant mouse. Non-stressed mice were sacrificed on the same day. Glucose concentrations from trunk blood samples were recorded using Accucheck C (Aviva) glucose meter and strips. Blood samples were also collected for analyses of plasma corticosterone (CORT) content. These samples were analyzed using a radioimmunoassay kit according to the manufactuerer's procotol (ICN Biomedicals, CA, USA). Inter assay variability was less than $10 \%$.

\subsubsection{Statistics}

All data were analyzed using $2 \times 2$ ANOVAs with drug ([D-Lys-3]-GHRP-6 vs $0.9 \%$ saline) and treatment (stress vs. non-stress) as the between group factors, unless otherwise stated. Data from animals with misplaced cannula were not included in the analyses. All analyses were preceded with a Leven's Test of Quality of Error Variance to ensure the homogeneity of variance assumption was satisfied. The limit for statistical significance was set at $\alpha=0.05$. All mice with mispalced cannula were eliminated from all statistical analysis.

\subsection{Results}

\subsubsection{Histology}

Postmortem analysis of cannulae placements showed that six mice had misplaced cannulae (vehicle non-stressed miss, $\mathrm{n}=1$; vehicle stressed miss, $\mathrm{n}=1$; [DLys-3]-GHRP-6 non-stressed miss, $\mathrm{n}=2$; [D-Lys-3]-GHRP-6 stressed miss, $\mathrm{n}$ $=2$ ). The location of the cannulae for animals in each group is shown in Figure 4.1. As seen in this figure, most placements were located mostly in the dorsolateral portion of the PVN. Because the cannula were inserted on an angle $\left(18^{\circ}\right)$, most missed cannulae were placed too far lateral to the PVN, with only a few placed too far dorsal. The data from these animals were not included in the analysis given that there were only one or two animals from each group that had misplaced cannulae. Nevertheless, none of the animals with misplaced cannulae and receiving [D-Lys-3]-GHRP-6 showed the effects observed below suggesting that these effects were caused by localized action of the drug on the PVN and not at sites outside this region. 


\subsubsection{Stress Increases Caloric Intake and This Effect is Not Attenuated by GHSR Antagonism in the PVN}

Total caloric intake was calculated daily throughout the study by adding the caloric content of the standard laboratory chow and high-fat diet. At the end of the baseline period, mice were assigned to each of the experimental groups after being matched for total caloric intake. As such, there were no group differences in the amount of calories consumed during the baseline (data not shown). During the week that followed the surgical implantation of the cannulae, mice continued to eat similar amounts of calories and there were no differences between any of the groups. During the stress period, there was no significant interaction between drug and treatment on caloric consumption [interaction, $\left.\mathrm{F}_{(1,22)}=0.019, p>0.05\right]$, nor was there an effect of [D-Lys-3]-GHRP-6 treatment [main effect of drug, $\left.\mathrm{F}_{(1,22)}=3.099, p>0.05\right]$. There was, however, a significant treatment effect where stressed mice increased their total daily caloric intake, and this effect was not attenuated by GHSR antagonism [main effect of stress, $\mathrm{F}_{(1,22)}=11.77, p<0.05$; See Figure 4.2A].

The increase in caloric intake seen in stressed animals was due primarily to an increase in the daily intake of regular chow. Both vehicle and [D-Lys-3]GHRP-6 treated mice consumed equivalent amounts of chow before the stress period began $(p>0.05)$. Following the stress period, there was no significant interaction between drug and treatment on the consumption of regular chow [interaction, $\left.\mathrm{F}_{(1,22)}=0.063, p>0.05\right]$. However, we did observe a significant treatment effect, where stressed mice rapidly increased their intake of regular laboratory chow regardless of the infusion they received in the PVN [main effect of stress, $\mathrm{F}_{(1,22)}=10.48, p<0.05$; See Figure 4.2B].

\subsubsection{Blocking GHSR in the PVN Increases the Intake of the High Fat Diet}

Given that stress has been associated with increased consumption of diets that are calorically dense and highly palatable, we next examined if chronic social defeat stress would alter the intake of a high-fat diet. Mice in all groups ate little of the high fat diet early in the baseline period but increased their intake steadily over time, so that by the end of the baseline period, they were consuming about $37 \% \pm 4.7$ of their daily caloric intake from the diet. There was no significant interaction between treatment and drug on the proportion of calories obtained from the high-fat diet during the stress period [interaction, $\mathrm{F}_{(1,22)}=$ $0.411, p>0.05]$. However, there was a significant treatment effect wherein the proportion of calories obtained from the high fat diet decreased in all animals that were stressed [main effect of stress, $\mathrm{F}_{(1,22)}=4.32, p<0.05$; data not shown]. Interestingly, there was also an effect of drug wherein mice receiving [D-Lys-3]-GHRP-6 infusions into the PVN increased the total amount of high fat diet consumed, relative to animals receiving vehicle infusions regardless of their treatment conditions [main effect for drug, $\mathrm{F}_{(1,22)}=4.25, p=0.051$; See 
Figure $4.2 \mathrm{C}]$.

The increase in the total amount of fat consumed by animals infused with [D-Lys-3]-GHRP-6 into the PVN was reflected in their body weight at the end of the stress period. There was no significant interaction between drug and treatment on the change in body weight during the stress period [interaction, $\mathrm{F}_{(1,22)}$ $=0.00, p<0.05]$ or on the average body weight at the end of the stress period [interaction, $\left.\mathrm{F}_{(1,22)}=0.425, p>0.05\right]$. There was, however, a significant effect of drug infusions on the change in body weight, wherein mice treated chronically with [D-Lys-3]-GHRP-6 weighed significantly more at the end of the stress period compared to animals treated with vehicle, regardless of their treatment group [main effect of drug, $\mathrm{F}_{(1,22)}=12.49, p<0.05$; See Figure 4.3A].

The effect of [D-Lys-3]-GHRP-6 on body weight were more pronounced when the data were expressed as a change from baseline, and a significant drug effect demonstrates that animals treated with [D-Lys-3]-GHRP-6 gained more weight than those treated with saline during the stress period [main effect for drug, $\mathrm{F}_{(1,22)}=7.32, p<0.05$; See Figure 4.3B]. Furthermore, there was a significant main effect of treatment on body weight gain. As expected, stressed mice gained significantly more weight than their non-stressed controls, however, this occurred independently of their drug treatment [main effect of stress, $\mathrm{F}_{(1,22)}=$ 4.65, $p<0.05$; See Figure 4.3B].

\subsection{4 [D-Lys-3]-GHRP-6 Infusions into the PVN Does Not Influence Stress-Induced Hyperglycemia but Does Mediate Stress-Induced CORT Secretion}

Following sacrifice by rapid decapitation, trunk blood was analyzed for blood glucose and corticosterone. There was no interaction between drug and treatment on circulating blood glucose at the time of sacrifice [interaction, $\mathrm{F}_{(1,22)}$ $=1.238, p>0.05]$. As expected, mice exposed to chronic social defeat stress had higher basal glucose levels at the time of sacrifice [Figure 4.4A; main effect of stress, $\left.\mathrm{F}_{(1,22)}=5.6, p<0.05\right]$, and this effect was not altered by [D-Lys3]-GHRP-6 infusions into the PVN $(p>0.05)$. Similarly, stressed mice had significantly higher levels of circulating CORT at the time of sacrifice compared to non-stressed controls, independent of drug treatment [main effect of stress, $\left.\mathrm{F}_{(1,22)}=12.263, p<0.01\right]$. Furthermore, animals receiving [D-Lys-3]-GHRP-6 infusions into the PVN tended to have higher circulating CORT at the time of sacrifice regardless of their treatment group [main effect of drug, $\mathrm{F}_{(1,22)}=4.188$, $p=0.058]$, and this main effect appeared to come from increased plasma CORT in samples collected from stressed [D-Lys-3]-GHRP-6 infused animals (Figure 4.4B). There was, however, no interaction effect between drug and treatment on circulating plasma CORT at the time of sacrifice [interaction, $\mathrm{F}_{(1,22)}=2.788$, $p=0.110]$. 


\subsection{Discussion}

Ghrelin is a peptide that acts centrally to increase food intake and to alter metabolism by promoting the oxidation of carbohydrates while sparing the oxidation of fatty acids as substrate, ultimately increasing adipose tissue accumulation (Tschöp et al., 2000). The effects of ghrelin on food intake and carbohydrate utilization have been mimicked by direct infusions of ghrelin onto the PVN (Currie et al., 2005). Similar to chronic delivery of ghrelin, acute and chronic social defeat stress increase the concentrations of the active form of ghrelin in plasma, but also result in higher caloric intake, and in some cases increased body weight, effects that are attenuated in socially defeated ghrelin receptor KO mice (Lutter and Sakata, 2008; Davies et al., 2009; Raspopow et al., 2010; Kumar et al., 2013). Interestingly, this type of social stress causes mice to alter their metabolism to promote the utilization of carbohydrates, ultimately leading to increased adipose stores (Davies et al., 2009). In contrast, ghrelin receptor $\mathrm{KO}$ mice continue to use fat as a substrate for nutrients and tend to lose body fat when chronically stressed, ultimately showing depletion of adipose tissue as described in Chapter 3. Furthermore, ghrelin seems to produce these metabolic effects centrally as chronic delivery of [D-Lys-3]-GHRP-6 into the cerebral ventricles decreases stress induced food intake and body weight gain.

In order to produce these effects, ghrelin may be targeting a number of central sites that contain the ghrelin receptor and that are involved in food intake and metabolic rate, as well as in the regulation of the stress response including the hypothalamic PVN (Guan et al., 1997; Zigman and Jones, 2006). The present study was designed to examine the contribution of ghrelin receptors in the hypothalamic PVN in modulating some of the metabolic effects caused by chronic social defeat stress in mice. The results from the current experiment indicate that chronic ghrelin receptor blockade in the PVN via infusions of [DLys-3]-GHRP-6, does not attenuate stress induced increases in caloric intake or body weight, nor does it affect plasma levels of glucose. Interestingly, while stress decreased the intake of a high fat diet that was provided for $4 \mathrm{~h}$ during the day, [D-Lys-3]-GHRP-6 treated mice tended to consume more of this diet compared to animals receiving vehicle infusions and this effect was independent from stress. Not surprisingly, [D-Lys-3]-GHRP-6 treated mice weighed more than vehicle treated mice. Furthermore, these animals also tended to have elevated levels of circulating CORT regardless of the treatment. These effects were not observed in the few animals that received [D-Lys-3]-GHRP-6 infusions into areas outside the PVN (i.e. missed cannula placements) supporting the evidence that GHSR signalling in the PVN selectively mediates these effects.

Given that acute direct infusion of ghrelin into the PVN increases food intake in rats (Currie et al., 2005) we expected that stress induced increases in caloric intake would be attenuated by chronic blockade of ghrelin receptors using [DLys-3]-GHRP-6. Nevertheless, [D-Lys-3]-GHRP-6 was ineffective in decreasing caloric intake. In contrast, work from Chapter 3 demonstrated that [D-Lys-3]GHRP-6 delivered into the ventricles while animals are being stressed does decrease stress induced caloric intake. It is therefore likely that when infused into 
the ventricles, [D-Lys-3]-GHRP-6 blocks ghrelin receptor in regions of the brain other than the PVN to reduce feeding including the ventromedial hypothalamus $(\mathrm{VMH})$ and brain stem nuclei like the parabrachial nucleus and nucleus of the solitary tract (NTS), all implicated in the feeding responses to ghrelin (Tschöp et al., 2000; Nakazato et al., 2001; Faulconbridge et al., 2003; Naleid et al., 2005; Abizaid and Liu, 2006; Skibicka et al., 2011). In this sense, central blockade of ghrelin receptors may attenuate the release of agouti related peptide (AgRP) and neuropeptide Y (NPY) from the ARC, attenuate dopaminergic tone from the VTA and noradrenergic tone from the brain stem to decrease food intake all resulting in overall attenuated feeding responses to chronic social stress. In our study, however, chronic blockade of the ghrelin receptor in the PVN was not sufficient to attenuate overall caloric intake.

In contrast, we did observe an increase in the intake of the palatable diet in those animals that received [D-Lys-3]-GHRP-6 into the PVN regardless of whether they were stressed or not. This would suggest that ghrelin receptors in the PVN, while not necessary for the overall feeding response seen during chronic social defeat, may be necessary for the switch in dietary preference that has been observed in stressed animals in previous studies. In these studies, mice with restricted access to a high fat diet to $4 \mathrm{~h}$ during the day gradually increase the proportion of calories consumed from this high fat diet while decreasing the proportion of calories consumed from a regular diet, indicating a bias toward the calorically dense and highly palatable diet. When stressed, however, these mice increased the intake of regular chow while decreasing the intake of the high fat diet. It is therefore possible that stress induced ghrelin secretion acts on the PVN to inhibit the intake of high fat without altering total caloric intake. This would be in accordance with data showing that the PVN is important for dietary preference for carbohydrates and lesions to the PVN result in increased preference for high fat diets (Aravich and Sclafani, 1983; Leibowitz, 1988; Hoebel et al., 1989). Interestingly, ghrelin decreases inhibitory post synaptic currents in parvocellular neurons within the PVN, primarily cells that secrete $\mathrm{CRH}$ (although also in some TRH and other cells of unknown phenotype), suggesting that ghrelin facilitates indirect activation of these neuroendocrine cell groups (Cowley et al., 2003). Furthermore, central ghrelin infusions increase extracellular norepinephrine content in the PVN (Kawakami, 2008). Of course these effects are acute and it is not known if they are the same in animals subjected to chronic stress. Thus, while plasma ghrelin and CORT levels correlate during acute stress, ghrelin secretion remains elevated during chronic stress but the CORT response becomes blunted (Zheng and Berthoud, 2008). Interestingly, our data shows that chronic GHSR antagonism in the PVN results in higher plasma CORT levels, an effect that seems more pronounced in stressed animals (although not statistically significant). This would suggest that ghrelin may be important in the adaptive mechanisms that bring CORT levels down in the face of chronic stress. As such, one would expect that chronic blockade of GHSR in the PVN would make mice more vulnerable to stress induced pathological conditions, a state previously shown in GHSR KO mice (Lutter and Sakata, 
2008).

Finally, it is important to note that in our studies cannulae placements were closer to the magnocellular portion of the PVN, where cells that produce oxytocin, vasopressin, and galanin are located (Akabayashi et al., 1994). All of these cell groups have been associated with feeding, and ghrelin may influence the expression of these peptides in the PVN directly or indirectly (Akabayashi et al., 1994; Sclafani et al., 2007; Beck and Max, 2008). Thus, one could argue that stress induced ghrelin secretion targets the PVN to alter the preference of high fat diets via modulation of oxytocin, vasopressin and galanin, a hypothesis that needs further study.

Possible limitations of this study are the use of [D-Lys-3]-GHRP-6 to antagonize the ghrelin receptor. For instance, [D-Lys-3]-GHRP-6 appears to interact with the $5-\mathrm{HT}_{2 b}$ receptors in the gut (Depoortere et al., 2006) although this interaction needs to be further demonstrated, as brain expression of this receptor is low compared to the gut (Hayes and Greenshaw, 2011). Given this finding, and the fact that other ghrelin receptor antagonists appear to act centrally to cause weight gain in spite of blocking the somatotropic effects of ghrelin (Halem et al., 2005), support the notion that the effects of [D-Lys-3]-GHRP-6, are related to their interaction with the ghrelin receptor and not to the other receptors.

In summary, our results further demonstrate that chronic social defeat stress results in increased caloric intake and preference for carbohydrate rich diets in mice. This preference for carbohydrate (but not the overall increase in caloric intake) is mediated by direct action of stress induced ghrelin secretion on the PVN. Blockade of the GHSR in the PVN biases animals toward increasing their intake of high fat, whether they are stressed or not. As such, the stress induced increases in caloric intake seen in vehicle treated mice, and the increases in the intake of the high fat diet are independent from each other and only the preference for fat is related to blockade of ghrelin receptors in the PVN. 
Figure 1

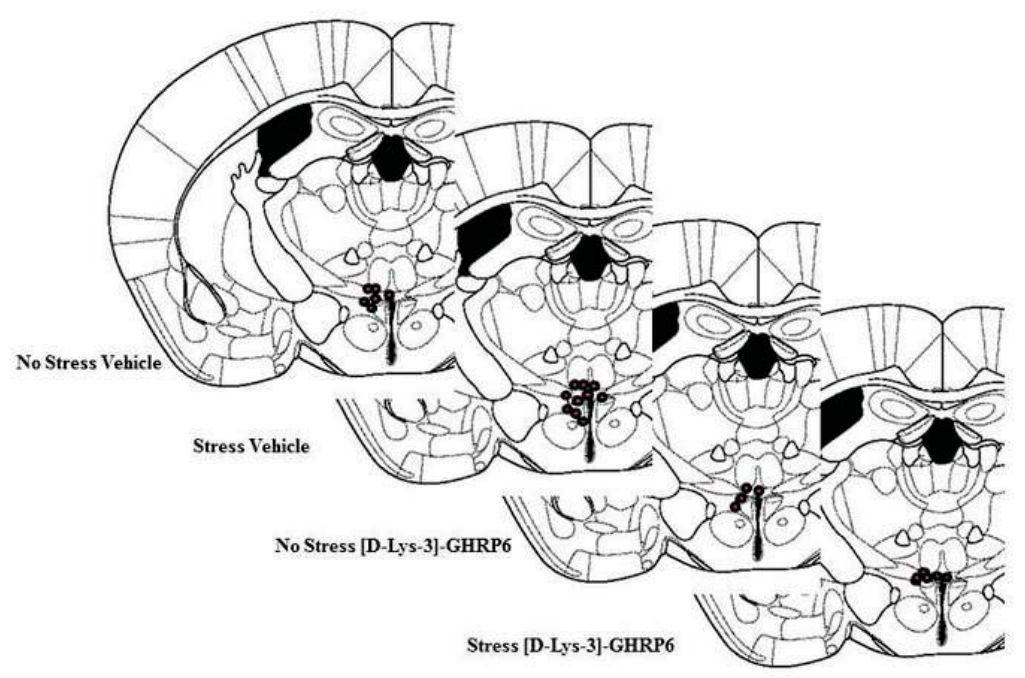

Figure 4.1: Location of cannulae placement in animals from each group. Animals with missed cannulae placements were not included in this figure or in the analysis 

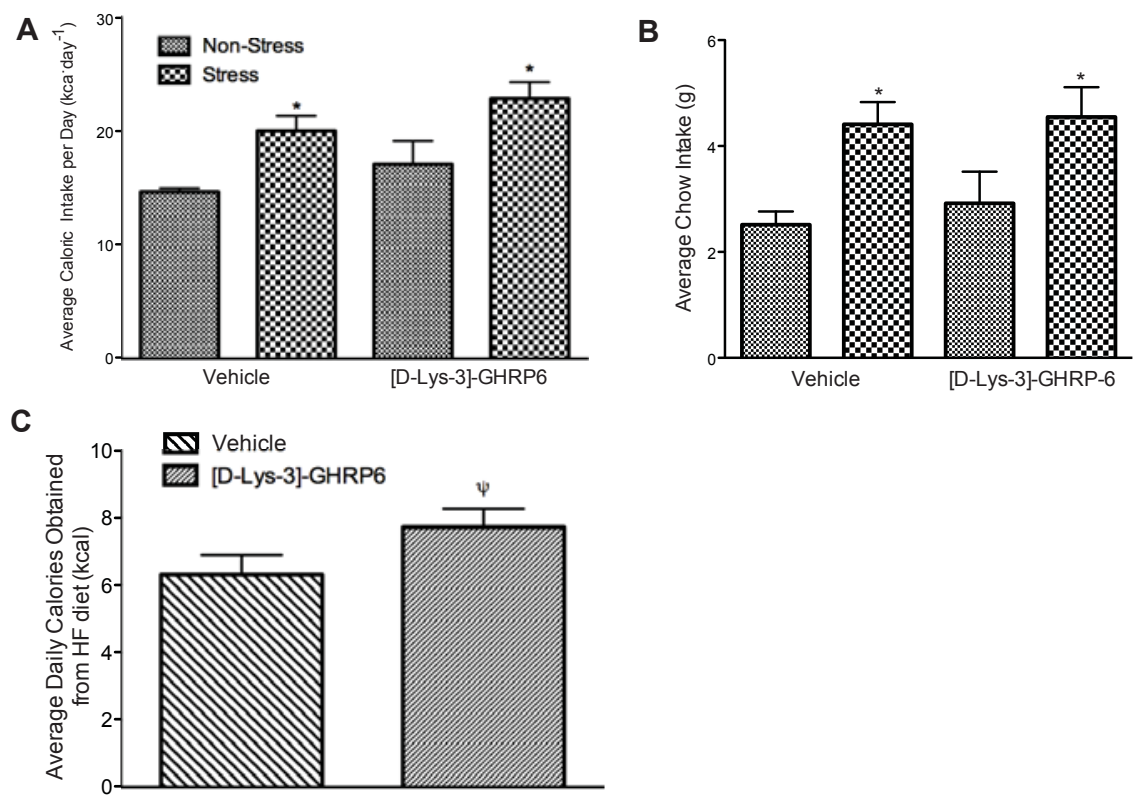

Figure 4.2: (A) Average daily caloric intake (calculated as the sum of calories consumed from standard laboratory chow and high-fat diet), (B) average daily standard laboratory chow intake, $(\mathrm{C})$ average number of calories consumed from the high fat diet per day for animals receiving either vehicle $(0.9 \%$ saline $)$ or 20nmol/day of [D-Lys-3]-GHRP-6; stressed and non-stressed animals are collapsed to illustrate main effect of drug. All values are expressed as mean \pm S.E.M. ${ }^{*} p<0.05$ relative to non-stressed controls. ${ }^{*} p=0.05$ relative to vehicle treated animals. 

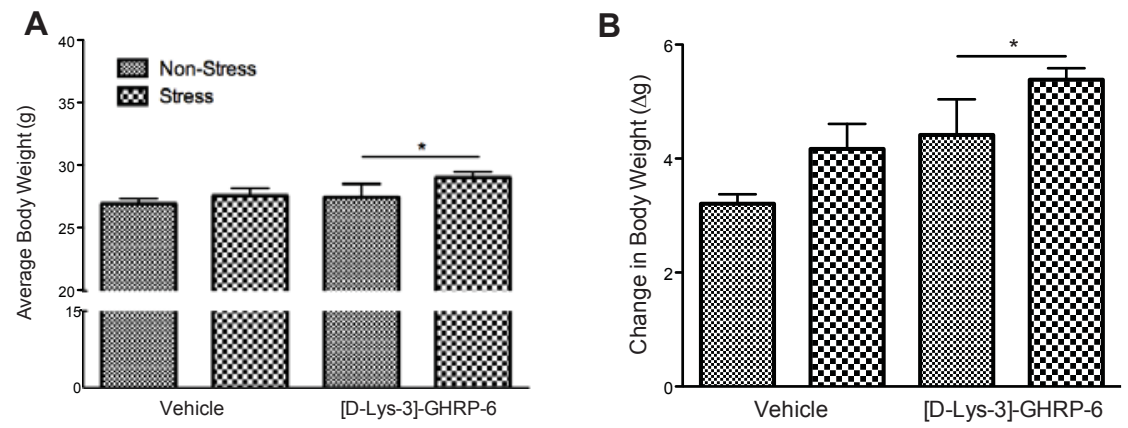

Figure 4.3: (A) Body weight at the end of the 3 week chronic social defeat paradigm, and (B) the change of body weight during the stress period. All values are expressed as mean \pm S.E.M. ${ }^{*} p<0.05$ relative to animals receiving vehicle infusions.

A

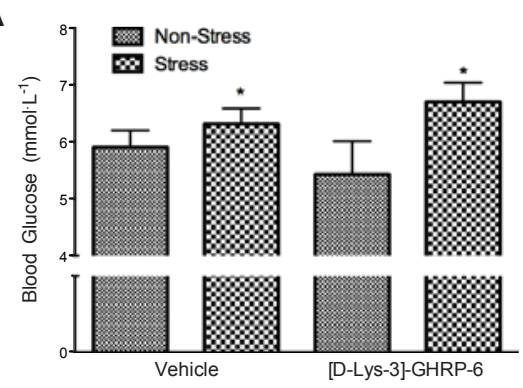

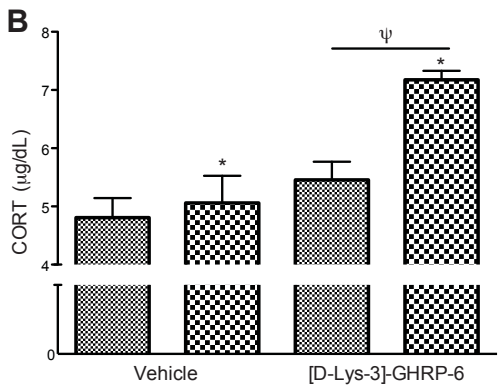

Figure 4.4: (A) Plasma blood glucose levels and (B) plasma corticosterone levels in trunk blood collected at the time of sacrifice. All values are expressed as mean \pm S.E.M. ${ }^{*} p<0.05$ relative to non-stressed animals; ${ }^{*} p<0.05$ relative to vehicle treated animals. 


\section{Chapter 5}

\section{Ghrelin Deficient Mice Have Fewer Orexin Cells \& Reduced cFos Expression in the Mesolimbic Dopamine Pathway Under a Restricted Feeding Paradigm}

E.W. Lamont, Z.R. Patterson, T. Rodrigues, O. Vallejos, I.D. Blum \&3 A. Abizaid

Neuroscience, 2012

doi: $10.1016 / \mathrm{j}$.neuroscience.2012.05.046

\subsection{Specific Aim 4}

It is possible that the effects of ghrelin on regulating the metabolic switch that occurs in response to stress is the direct result of signalling cascade activation downstream of GHSR-1a, and subsequent changes in protein transcription/translation, fatty acid oxidation, growth hormone secretion, etc. However, ghrelin's actions may also include the direct and indirect recruitment of orexin neurons in the lateral hypothalamus, an area known for its ability to promote food intake and food-motivated behaviors. Indeed ghrelin causes direct activation of cFos immunoreactivity in isolated orexin neurons (Lawrence et al., 
2002) and ghrelin's antidepressant like effects following an acute food restriction stressor depends on the presence of orexin neurons. There is also evidence demonstrating that ghrelin positive neurons project directly to the LH, and that these neurons directly modulate VTA dopamine neurons to mediate feeding behaviours (Borgland et al., 2006, 2009). These data suggest these two systems operate in parallel to mediate the physiological response to acute food restriction and to promote motivated feeding behaviors. However, it is unknown whether the orexin system is involved, or recruited by ghrelin to mediate the metabolic changes that occur in response to stress. The orexin pathway is capable of responding to changes in ghrelin secretion and has been shown to elicit orexigenic and adipogenic responses in rodents. Therefore, we hypothesized that a lack of ghrelin signalling would down-regulate the orexin system in areas of the brain that are critical to feeding behaviour (i.e VTA, LH). To this end, we examined the relative co-expression of orexin and GHSR-1a in many stress and feeding related areas of the brain. Furthermore, we exposed both WT and GHSR KO mice to a food restriction stress paradigm and compared the neuronal activation of orexin-positive neurons in these critical brain regions.

\subsection{Introduction}

Stress is defined as the behavioural and physiological changes that arise in the face of, or in anticipation of a perceived threat. One of the most notable changes that arises as a consequence of stress is a change in an organisms metabolism. These metabolic changes result in an increase in food intake, body weight and adipose tissue, and have been associated with the development of obesity, cardiovascular disease and metabolic syndrome. Work presented in this thesis suggests that stress promotes caloric intake as a protective mechanism to ensure that the organism has adequate energy supplies necessary to deal with the stressor at hand. Chronic stress, however, leads to prolonged activation of the stress response and the once protective mechanisms eventually become detrimental. The stress response, as described in Chapter 1, terminates with the secretion of glucocorticoids into the general circulation. Until recently, it was thought that glucocorticoids served as a signal promoting the intake of calorically dense comfort foods (Dallman and Pecoraro, 2003; Dallman et al., 2005) and promoting the storage of fat tissue, ultimately shaping an organisms phenotype in response to stress. More recent work, however, has demonstrated the role of other metabolically active hormones in the physiological response to stress. One of those hormones, as outlined in Chapters 1-5 is the peripherally derived hormone ghrelin. Work presented in this thesis suggests that ghrelin plays an important role in mediating stress-induced increases in both food intake and body weight, as interruption of ghrelin signalling blunts this effect. Furthermore, central ghrelin signalling is sufficient to dictate the real-estate from which an organism gathers its energy resources. Specifically, ghrelin promotes the utilization of carbohydrates as a rapid fuel source, while sparing the breakdown of adipocytes, a potential role of ghrelin signalling in the PVN. What remains 
to be fully understood is whether intracellular signalling cascades downstream of GHSR-1a activation (i.e. AMPK signalling cascade) are solely responsible for mediating the metabolic changes associated with stress-induced ghrelin secretion, or whether activation of GHSR-1a results in the recruitment of other parallel circuits.

The orexin/hypocretin system is one potential pathway recruited by GHSR-1a activation in the context of increasing food intake and promoting food motivated behaviours. Ghrelin activation of arcuate neurons leads to the release of NPY onto lateral hypothalamic orexin neurons, producing excitatory responses in these cells and subsequent food intake (Olszewski et al., 2003; Yamanaka et al., 2003). Interestingly, the orexigenic effects of orexin are thought to be closely related to the actions of NPY, as their exists reciprocal connections between LH orexin neurons and ARC NPY neurons. These same NPY neurons show a dense expression of GHSR-1a and are known to be stimulated in the presence of ghrelin (Howard et al., 1996; Lucidi et al., 2005; Willesen et al., 1999; Zigman and Jones, 2006).

The orexin system projects widely throughout the CNS and has been heavily implicated in responding to rewarding stimuli, both natural and artificial, with particular attention paid its role in food motivated behaviours. Indeed, central administration of orexin is sufficient to increase food intake and interruption of orexin neurostransmission causes a reduction in food intake. Orexin's ability to mediate reward-oriented behaviours are a product of connections made with the VTA dopaminergic cells (Borgland et al., 2006, 2009). Orexin administration directly into the VTA causes significant firing of VTA dopamine neurons and orexin-receptor antagonist blunt drug induced activation of this system (Borgland et al., 2006). The same phenomena has been described within the context of ghrelin. Ghrelin administration directly into the VTA increases food intake, and blocking ghrelin signalling decreases food intake (Abizaid and Liu, 2006; Skibicka et al., 2011). Furthermore, it has been shown that rats will show increased motivation to obtain a food reward as a consequence of ghrelin signalling in the VTA (King et al., 2011). The strong reciprocal connections, and similar physiological functions between ghrelin and orexin lead to the hypothesis that ghrelin may in fact recruit the orexin system in response to a stressor. To this end, we exposed WT and GHSR KO mice to a chronic food restriction stressors and measured the relative activation of orexin neurons in the LH as well as dopamine neurons in the VTA. Food restriction is known to increase ghrelin levels, and animals require circulating ghrelin in order to anticipate meal presentations during a food restriction paradigm (Blum et al., 2009). Furthermore, food restriction has been shown to upregulate stress responsive genes, increase circulating CORT levels and promote the development of stress-induced ulcers (Guarnieri et al., 2012; Pesic et al., 2010; Savard et al., 1983; Nakamura et al., 1990).

Results indicate that ghrelin promotes food anticipation in part through its actions on midbrain and hypothalamic pathways associated with reward and arousal. GHSR-1a expression in the LH is important for producing food motivated behaviours in response to food restriction stress, and therefore may be 
involved in stress-induced metabolic disturbances.

\subsection{Materials \& Methods}

\subsubsection{Animals}

Male mice with targeted mutations to the ghrelin (ghrelin KO) and ghrelinreceptor genes (GHSR KO) and their WT littermates were bred at the Carleton University Department of Neuroscience animal facilities. Animals were approximately 3-4 months of age at the beginning of the experiment. Both types of knock-out mice originated from heterozygous breeding pairs obtained from Regeneron Pharmaceuticals (Tarrytown, NY, USA). These mice were generated using a mixed C57BL/6J and DBA strain as background and the coding region of the GHSR locus or the GHRL locus, for GHSR KO and ghrelin KO respectively, was deleted and replaced with an in-frame lacZ reporter gene (Wortley et al., 2005; Abizaid and Liu, 2006; Diano et al., 2006). All animals were housed in standard mouse cages (Nalgene) and received ad libitum chow (unless otherwise specified) and water in a temperature and humidity-controlled vivarium with a 12-h lightdark schedule (L:D), with lights on at 8:00AM. All manipulations were conducted under the guidelines of the National Institute of Health (NIH) and Canadian Council on Animal Care and approved by the Animal Care Committees of Carleton and Yale Universities.

\subsubsection{Restricted Feeding Paradigm}

The time course of the experiment is shown in Figure 5.1. GHSR KO and WT mice were placed on a RF schedule for 7 days. Animals were given a daily scheduled meal from noon to 4:00PM for 6 days. On the last day, animals were sacrificed in the 2-h period prior to the anticipated mealtime, between 10:00AM and 12:00PM.

\subsubsection{Immunocytochemistry}

Mice were injected with an overdose of sodium pentobarbital and perfused with $100 \mathrm{ml}$ of saline $(0.9 \%)$ followed by $200 \mathrm{ml}$ of $4 \%$ paraformaldehyde. Every fourth $60 \mu \mathrm{m}$ section through the NAc and VTA were processed for immunocytochemistry using a cFOS antibody (Oncogene Science, Boston, MA, USA), as described previously (Abizaid and Liu, 2006). Briefly, free-floating sections were washed in $0.1 \mathrm{M}$ phosphate-buffered saline (PBS), $\mathrm{pH} 7.4$, and incubated at room temperature (RT) for 30 minutes in a quenching solution made of PBS and 1\% $\mathrm{v} / \mathrm{v} \mathrm{H}_{2} \mathrm{O}_{2}$. After the quenching phase, sections were rinsed in cold PBS and incubated for 1 hour at RT in a pre-blocking solution made of $0.3 \%$ Triton X-100 in PBS and BSA. After the pre-blocking phase, sections were transferred directly into an affinity-purified rabbit polyclonal antibody raised against cFOS diluted 1:20,000 with a solution of Triton X-PBS with BSA. Sections were incubated with the primary antibody for 48 hours at $4^{\circ} \mathrm{C}$ then rinsed in cold PBS and 
incubated for 1 hour at RT with a biotinylated anti-rabbit IgG made in donkey (Jackson ImmunoResearch Laboratories, Inc., Westgrove, PA, USA), diluted 1:250 with BSA in PBS. After incubation with secondary antibody, sections were rinsed in cold PBS and incubated for 1 hour at RT with an avidinbiotinperoxidase complex (Vectastain Elite ABC Kit; Vector Labs). Finally, sections were rinsed with PBS and rinsed for 5 minutes with $0.05 \%$ 3,3'-diaminobenzidine $(\mathrm{DAB})$ in PBS. Sections were then incubated on an orbital shaker for 5 minutes in $\mathrm{DAB}$ with $0.01 \% \mathrm{H}_{2} \mathrm{O}_{2}$ and cobalt chloride. After this final incubation, sections were rinsed in cold PBS, wet-mounted onto gel-coated slides, dehydrated through a series of alcohols, soaked in Clearene (Surgipath, Leica Microsystems Inc., Concord, ON, Canada), and cover-slipped with Permount (Fisher Scientific, Toronto, ON, Canada). For cFOS/orexin B double-labeled tissue, separate sections through the LH from the same GHSR KO and WT mice and were processed for cFOS as described above, then again for orexin B (goat anti orexin B IgG, 1:10,000, Santa Cruz Biotechnology Inc., Santa Cruz, CA, USA). After being processed for cFOS, sections were rinsed in PBS then incubated with orexin B for 48 hours at $4^{\circ} \mathrm{C}$, rinsed in cold PBS and incubated for $1 \mathrm{~h}$ at RT with a biotinylated anti-goat IgG made in donkey (Jackson; 1:250), rinsed and incubated for 1 hour at RT with an avidinbiotin-peroxidase complex (Vector Labs). Finally, sections were rinsed with PBS, rinsed for 5 min with $0.05 \%$ DAB in PBS, then incubated for 30 minutes in DAB with $0.01 \% \mathrm{H}_{2} \mathrm{O}_{2}$. After this final incubation, sections were rinsed in cold PBS, wet-mounted onto gelcoated slides, dehydrated through a series of alcohols, soaked in Clearene, and cover-slipped with Permount. Sections through the LH from ghrelin KO and $\mathrm{WT}(\mathrm{n}=8 /$ group) mice were stained for orexin B as described above.

\subsubsection{Data Analysis}

Brain tissue sections were examined using an Olympus BX51 bright field microscope (Olympus Canada, Markham, ON, Canada) and associated digital capture device DVC-2000C (DVC Company, Austin, TX, USA) connected to a desktop computer with Windows XP operating system (Microsoft Corp., Redmond, WA, USA). Cells immunopositive for $\mathrm{cFOS}$ and orexin B were counted manually using the captured images using Image XSM software (v. 1.91, 2010, www.liv.ac.uk/ sdb/ImageSXM/). For analysis, the mean number of cFOS and orexin B immunoreactive (IR) cells per region was calculated for each animal from the counts of four unilateral images showing the highest number of labeled cells. Differences between KO and WT group means were assessed using T-tests (Prism 5 for Mac OSX, v. 5.0c, 2009, GraphPad Software Inc., La Jolla, CA, USA), with significance threshold set at $p<0.05$ for all analyses. Subjects with an average cell count more than 5 standard deviations outside of the mean for their group were excluded from the analysis. 


\subsection{Results}

\subsubsection{GHSR KO Animals Show Attenuated cFOS Expres- sion in Major Regions of the Mesolimbic DA Path- way in Anticipation of a Scheduled Meal}

As can be seen in Figure 5.2, the expression of cFOS in the VTA was significantly lower in aniticpation of a scheduled meal in GHSR KO $(\mathrm{n}=3)$ mice relative to WT $(\mathrm{n}=4)$ mice $(\mathrm{T}[5]=2.7, p<.05)$. In the VTA, GHSR KO mice had only $21 \%( \pm 3.6 \%)$ of the number of cFOS-IR cells observed in WT animals $(100 \% \pm 24.4 \%)$. An examination of some of the major projection areas of the VTA DA neurons, the dorsal and ventral striatum, shown in Figure 5.3, reveals that the expression of cFOS in the NAc shell was also lower in GHSR KO compared to WT mice, with KO animals expression $37 \%( \pm 11.7 \%)$ the number of cFOS-IR cells observed in WT animals $(100 \% \pm 26.4 \%$; T[7] $=2.0$, $p<.05)$, but cFOS expression in the NAc core $(73 \% \pm 21.5 \%$ in GHSR KO mice, vs. $100 \% \pm 17.6 \%)$ and in the caudate/putamen $(\mathrm{CPu})(64 \% \pm 23.8 \%$ in GHSR KO mice, vs. $100 \% \pm 64.0 \% ; p>.05)$ did not differ by genotype $(p>$ $.05)$.

\subsubsection{Scheduled-Feeding Induces Activation of Orexin Neu- rons That is Reduced in GHSR KO Animals}

Figure 5.4 shows sample images from the LH of GHSR KO and WT mice sacrificed just prior to their scheduled meal. As seen in the figure, the total number of cFOS-IR cells (including orexin B-IR and non-orexin cells) significantly differed between the two genotypes $(\mathrm{T}[11]=2.5, p<.05)$. The number of double-labeled orexin B/cFos-IR cells also differed significantly after 7 days of daily food access during the middle of the day $(\mathrm{T}[11]=2.7, p<.05)$, which also corresponded to a significantly greater proportion of double-labeled cells, as a percentage of the number of orexin B-IR cells in KO $(42 \% \pm$ SEM 5.6\%) relative to WT $(25 \%$ \pm SEM $4.7 \%)$ mice $(\mathrm{T}[11]=2.2, p<.05)$. Interestingly, GHSR KO animals showed a trend for a reduced number of orexin B-IR cells $(\mathrm{T}[11]=1.4 ; p=$ $0.095)$, such that GHSR KO animals had $77 \%( \pm 14 \%)$ of the number of orexin cells observed in WT animals $(100 \% \pm 9.4 \%)$.

\subsubsection{The Number of Orexin Neurons in the LH is Re- duced in Ghrelin KO Mice}

The previous experiment suggested that ghrelin is important for the full expression of the orexin protein in the LH of mice. To determine this, mice with targeted deletion of the ghrelin gene were sacrificed and processed for immunocytochemistry as described above and the number of cells expressing orexin B was counted and analyzed to determine basal differences between ghrelin $\mathrm{KO}$ mice and their WT litter mates. As seen in Figure 5.5, the number of orexin-BIR cells is significantly reduced relative to WTs $(\mathrm{T}[13]=2.6, p<.05)$, such that 
ghrelin KO animals had $75 \%( \pm 8.5 \%)$ of the number of orexin cells observed in WT animals $(100 \% \pm 5.2 \%)$.

\subsection{Discussion}

In the present study, we examined the role of ghrelin in activating areas of the brain related to reward and arousal including the VTA, NAc core and shell, dorsal striatum and LH, in anticipation of a scheduled meal. Our findings confirm that cFOS expression is elevated in anticipation of a scheduled meal in some regions of the mesolimbic reward pathway in WT mice, and this effect is attenuated in GHSR KO mice. These effects were observed in the VTA and the shell portion of the NAc, a terminal field to VTA dopaminergic neurons (Wise, 2004, 2005). In contrast to the dorsal striatum, the $\mathrm{CPu}$, which primarily receives projections from the DA cells of the substantia nigra (Wise, 2004), had similar levels of cFOS expression in both genotypes in anticipation of the scheduled meal. These findings are consistent with previous studies showing that a RF schedule can increase daytime cFOS expression in motivation-related brain regions (Angeles-Castellanos et al., 2007). In fact Angeles-Castellanos et al. (2007) demonstrated that the NAc core and shell regions have a circadian rhythm of cFOS expression that is shifted in response to a RF schedule, such that the peak level that normally occurs during the middle of the night (active period) under ad libitum feeding conditions, is dramatically increased during the daytime, with a time of peak occurring around mealtime. Our data show, however, that this daytime increase in the level of cFOS expression in the VTA and NAc shell may be related in part to high ghrelin levels during the period immediately prior to scheduled arrival of the meal. Indeed, a number of studies show that total and active ghrelin concentrations are elevated in anticipation of scheduled meals and that these plasma concentrations correlate with both anticipatory activity as well as with cFOS expression in a number of hypothalamic regions (Drazen et al., 2006; Blum et al., 2009; LeSauter et al., 2009). Moreover, GHSR mutation leads to attenuated cFOS expression in these hypothalamic regions (Blum et al., 2009), and as seen in our study, attenuated cFOS expression in the VTA and shell portion of the NAc. While GHSR is found in cells that project to the NAc core and dorsal striatum, we found no differences between GHSR KO and WT mice in the activity of these regions when anticipating a scheduled meal. This suggests that neuronal activation in these regions is driven by factors other than ghrelin. Alternatively, activation of the NAcc core may reflect behaviors other than food anticipation. Indeed, the careful analysis by Angeles-Castellanos et al. (2007) clearly shows that while the peak of cFOS expression in the NAc shell is at the time of meal presentation, the same time point used in our experiment, the NAc core showed the greatest number of cFOS-IR cells after mealtime (Angeles-Castellanos et al., 2007). A study examining the time-course of cFOS expression in GHSR KO animals on an RF schedule would allow for a finer discrimination of changes in level and 
timing of activation in the NAc on this type of feeding schedule.

The effects of ghrelin on feeding have been attributed in part to the stimulation of orexin neurons in the LH. Injections of ghrelin induce a greater level of cFOS expression in the LH, particularly in orexin (but not $\mathrm{MCH}$ ) neurons (Lawrence et al., 2002; Toshinai et al., 2003; Blum et al., 2009). In addition, we demonstrated that GHSR KO animals showed fewer cFOS-IR cells in the LH, relative to WT mice, in response to 2 weeks on a temporally RF schedule and when sacrificed just prior to the scheduled feeding time (Blum et al., 2009). In the present study we also found that GHSR KO mice showed a lower number of cFOS-IR cells than WT mice after 7 days on an identical RF feeding schedule. In addition, we also observed a significantly reduced number of double-labeled orexin/cFOS-IR cells. In WT animals, the proportion of orexin-IR neurons double-labeled with cFOS was $42 \%$ versus only $25 \%$ in GHSR KO mice at ZT4. Moreover, and unexpectedly, we also found that mice with mutations to either the ghrelin or GHSR gene tended to have lower number of orexin-IR cells in the LH. Indeed we found that the number of orexin cells in GHSR KO mice was approximately $77 \%$ that of the WT animals. A follow up study using non-food-restricted ghrelin KO mice confirmed that the absence of ghrelin was correlated with lower orexin immunoreactivity with ghrelin KO mice having approximately $75 \%$ of the number of orexin-IR cells, as compared to WT mice. As such, these data suggest that orexin cells in the LH are important targets for ghrelin to promote anticipation. This idea is supported by studies showing that orexin-incompetent mice show attenuated food anticipatory locomotor activity to scheduled meals (Akiyama et al., 2004; Mieda et al., 2004; Kaur et al., 2008; Gunapala et al., 2011). But perhaps more interestingly, the data suggest that ghrelin is important for the development of the orexin system, a question that needs to be addressed in future studies.

An important alternative explanation to consider is whether the reduced number of orexin B-IR cells in ghrelin KO and GHSR KO mice may be responsible for the reduced cFOS-IR observed in the VTA and NAc shell. Orexin neurons in the LH modulate the midbrain DA reward system (Borgland et al., 2006) at the level of the VTA (Fadel and Deutch, 2002), and NAc shell (Mori et al., 2011). In fact, orexin B stimulates almost $80 \%$ of neurons in the NAc shell (Mori et al., 2011). If orexin has such a powerful effect on the NAc shell, then even a small reduction in orexin cells could attenuate a food restrictioninduced increase in activity. It is difficult to separate the contribution of ghrelin vs. orexin in this type of situation, since there are so many similarities in terms of their behavioral effects. Both GHSR KO and orexin KO mice show less effect of the arousing effects of short term food-deprivation (Yamanaka et al., 2003; Esposito et al., 2012), and the effects of these two mutations on the synchronization of anticipatory activity to RF are also similar (Akiyama et al., 2004; Mieda et al., 2004; Kaur et al., 2008; Blum et al., 2009; LeSauter et al., 2009). Clearly some more research is needed to understand the interaction of orexin and ghrelin systems in arousal and RF. 


\subsection{Conclusions}

These experiments support a growing body of literature advancing the synergistic relationship between ghrelin and orexin in coordinating metabolism, reward, and arousal. Orexin is critical for both arousal and motivation and absence of orexin, either due to genetic deletion or disease, causes narcolepsy, a sleep disorder characterized by profound disturbances of sleep and arousal (Tsujino and Sakurai, 2009). In addition, orexin ablation leads to marked motivational and metabolic disturbances (Tsujino and Sakurai, 2009; Thompson and Borgland, 2011). Finally, ghrelin, which is primarily known as an orexigenic hormone, has also been shown to have effects on sleep and arousal. Injections of ghrelin directly into the LH induced feeding and wakefulness (Szentirmai et al., 2007), and GHSR KO animals show a lack of arousal in response to 48 hours fooddeprivation (Esposito et al., 2012). Further, there are studies suggesting that ghrelin's effects on food reward, may be mediated through the orexin pathway, given that numerous ghrelin-mediated effects are absent in orexin KO mice (Lutter and Sakata, 2008; Lutter et al., 2008; Perello et al., 2010). In general, our data support the idea that ghrelin promotes food anticipation in part through its actions on midbrain and hypothalamic pathways associated with reward and arousal. Receptors for ghrelin in these regions are therefore important for the full expression of food anticipation, and animals with a mutation to the GHSR gene may show a diminished motivation to engage in activities related to food seeking, a state that may be maladaptive in nature, but perhaps useful when designing drugs aimed at curbing appetite. 


\begin{tabular}{|c|c|c|c|c|c|c|c|}
\hline Baseline & Day 1 & Day 2 & Day 3 & Day 4 & Day 5 & Day 6 & Day 7 \\
\hline Ad llbltum food access & & & & & & & \\
\hline
\end{tabular}

Figure 5.1: A schematic diagram showing the time course of the experimental design. Prior to the beginning of the experiment, all mice had ad libitum access to standard laboratory chow and tap water. Mice with a targeted deletion of the ghrelin receptor (GHSR KO animals) and wild type (WT) mice were placed on restricted feeding schedule (RF) for 7 days. Animals were given a daily scheduled meal from noon-4:00PM for 6 days (indicated in gray). On the 7 th day, animals were sacrificed in the 2 hour period prior to anticipated mealtime, between 10:00AM \& 12:OOPM 

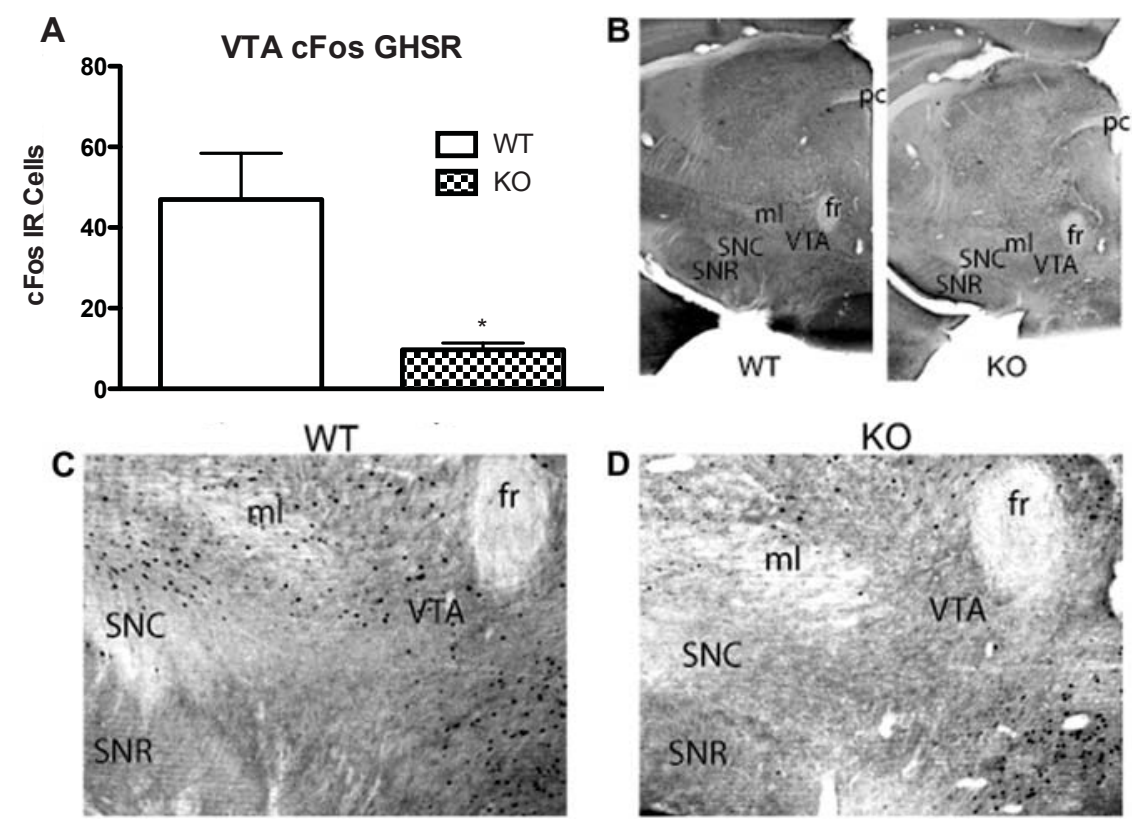

Figure 5.2: The expression of cFOS in the ventral tegmental area (VTA) was reduced at 7 days of restricted feeding in GHSR KO animals, compared to WT mice. (A) Mean \pm standard error of the mean (SEM) number of cFOS immunoreactive (IR) nuclei in the VTA as a function of genotype. * indicates a significant difference from WT $(p<.01 ; \mathrm{n}=4$ per group). (B) Photomicrographs showing examples of cFOS expression in the VTA of WT (left) and $\mathrm{KO}$ (right) animals. Magnification 2.5×. (C) Photomicrographs showing examples of cFOS immunoreactivity in the VTA of WT mice, and (D) KO mice. Magnification $10 \times$. 


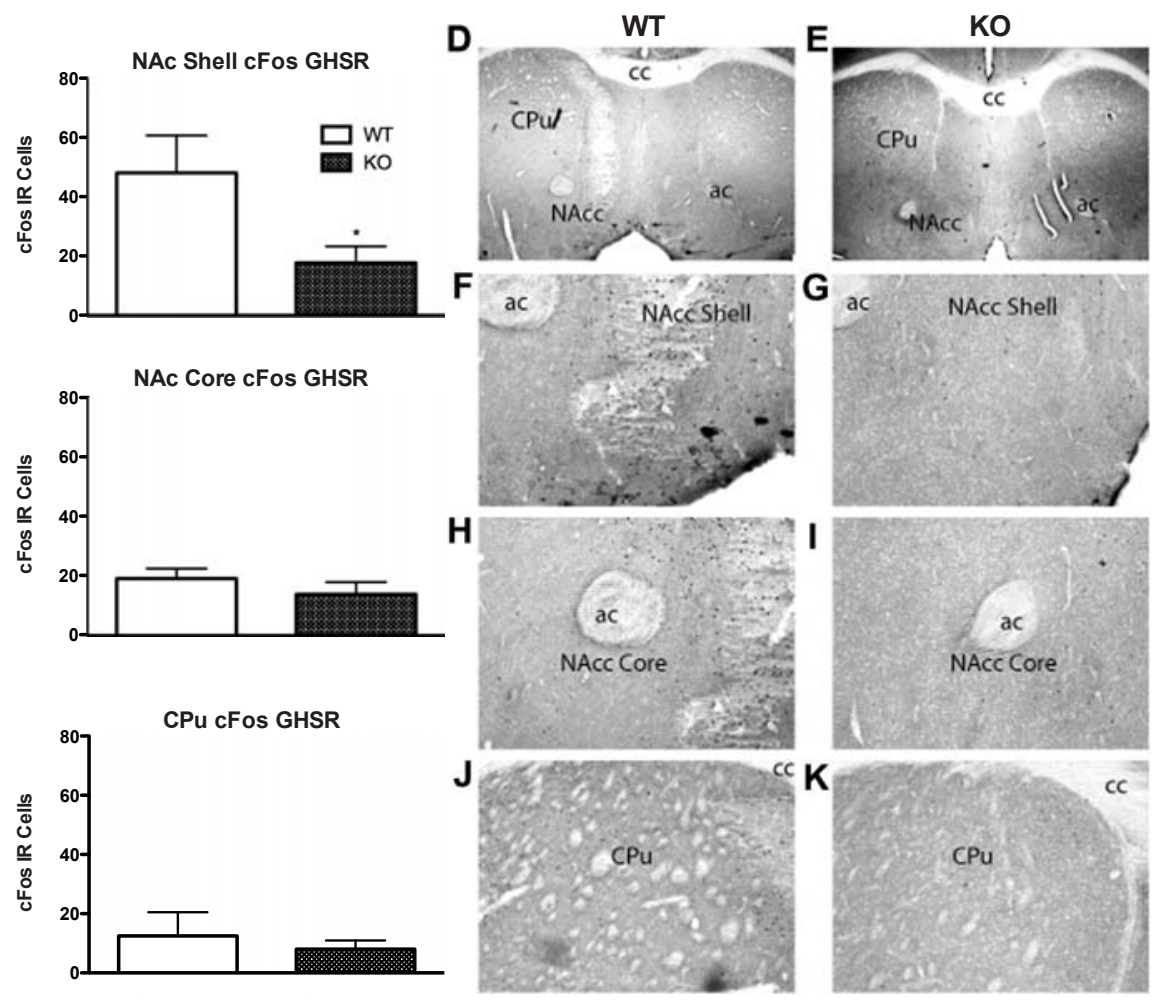

Figure 5.3: The expression of cFOS in the nucleus accumbens shell (NAc shell) was reduced after 7 days of restricted feeding in GHSR KO animals relative to WT mice. (A) Mean \pm SEM number of cFOS-IR nuclei in the nucleus accumbens shell (NAc shell); and (B) NAc core, and (C) caudate putamen $(\mathrm{CPu})$ as a function of genotype. ${ }^{*}$ indicates a significant difference between GHSR KO and WT in the NAc shell only ( $p<.05 ; \mathrm{n}=4-5$ per group). (D) and (E) Photomicrographs showing examples of cFOS expression in the striatum of WT (D) and KO (E) animals. Magnification 2.5×. (F) and (G) Photomicrographs showing examples of cFOS immunoreactivity in the NAc shell of WT $(G)$ and $\mathrm{KO}(\mathrm{G})$ mice; $(\mathrm{H})$ and $(\mathrm{I})$ show cFOS-IR in the NAc Core of WT $(\mathrm{H})$ and $\mathrm{KO}$ (I) mice; and $(\mathrm{J})$ and $(\mathrm{K})$ show cFOS-IR in the $\mathrm{CPu}$ of WT $(\mathrm{J})$ and $\mathrm{KO}(\mathrm{K})$ mice. Magnification $10 \times$. 
A
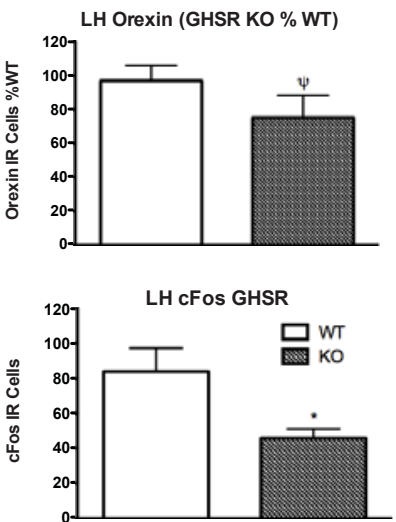

C

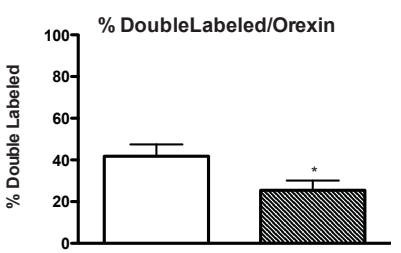

WT

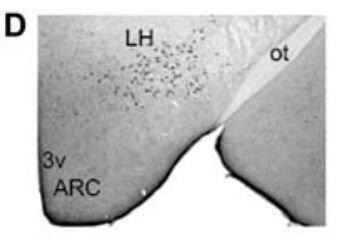

F
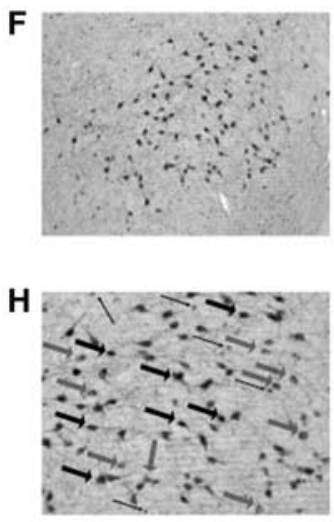

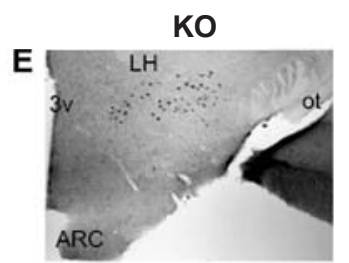

G

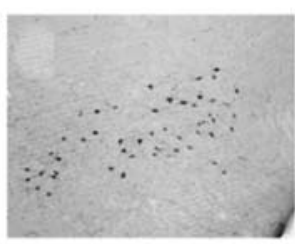

I

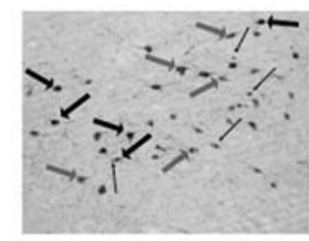

Figure 5.4: The expression of orexin, cFos, and double-labeled cells in the lateral hypothalamus (LH). GHSR KO animals show a trend for a reduced number of orexin cells, and a smaller number of total cFOS and double-labeled orexin/cFOS-IR cells after 7 days of daily food access during the middle of the day. (A) Mean \pm SEM number of orexin-IR cells in the LH as a function of genotype, expressed as a percentage of WT expression levels. Symbol indicates a trend for fewer orexin-IR cells in KO mice $(p<0.1 ; \mathrm{n}=67$ per group). (B) Mean SEM number of cFOS-IR nuclei in the LH was significantly reduced in GHSR KO mice compared to WT ( $p<.05 ; \mathrm{n}=67$ per group). (C) Mean $\pm \mathrm{SEM}$ cells that are immunoreactive for both orexin and cFOS, expressed as a percentage of the number of orexin-IR cells. ${ }^{*}$ Indicates fewer orexin/cFOS-IR cells in KO mice ( $p<.05 ; \mathrm{n}=67$ per group). (D) and (E) Photomicrographs showing examples of cFOS and orexin-immunoreactive expression in the LH of WT (D) and KO (E) animals. Magnification 2.5×. (F) and (G) Examples of WT (F), and $\mathrm{KO}(\mathrm{G})$ mice at $10 \times$ magnification. $(\mathrm{H})$ and $(\mathrm{I})$ show detail of cFOS, orexin, and double-labeled cells in WT (H) and KO (I) mice. Small black arrows show examples of cells positive for cFOS, large gray arrows indicate orexin-IR cells, and large black arrows indicate double-labeled cells. Magnification $10 \times$ 

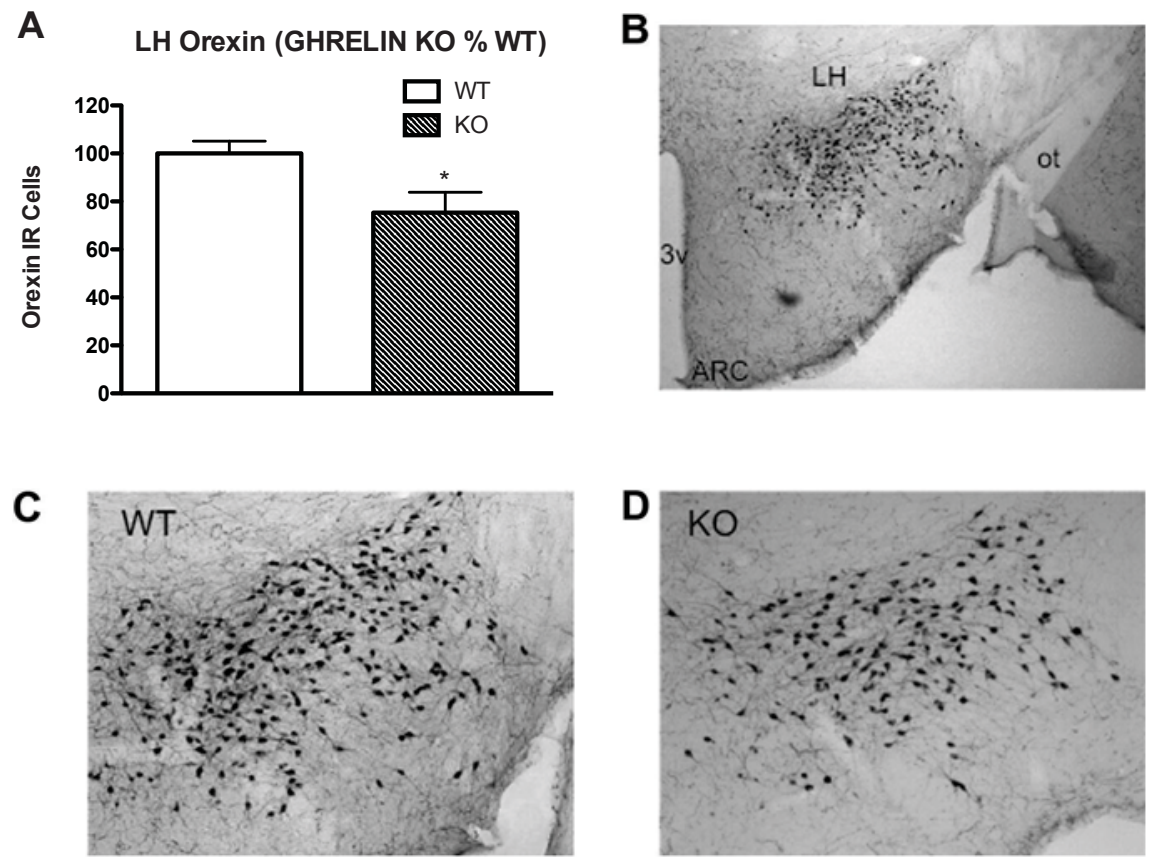

Figure 5.5: The expression of orexin is reduced in the lateral hypothalamus of freely fed animals with a targeted knock out of the ghrelin gene. (A) Mean \pm SEM number of orexin-immunoreactive nuclei in the LH expressed as a percentage of the average number of orexin-IR cells in the WT group.* Indicates a significant difference from the wild type (WT; $p<.05 ; \mathrm{n}=78$ per group). (B) Photomicrographs showing examples of orexin expression in the LH. Magnification 2.5×. (C) and (D) Photomicrographs showing examples of orexin immunoreactivity in the LH of WT (C), and KO (D) mice. Magnification 10× 


\section{Chapter 6}

\section{Putative GOAT Inhibitor, CF801, Prevents Stress Induced Ghrelin Secretion \& Subsequent Increases in Caloric Intake}

Zachary R. Patterson, Martin Wellman, Harry MacKay, Trevor Rodrigues 83 A. Abizaid

Manuscript in preparation

\subsection{Specific Aim 5}

Chronic social defeat stress is associated with increased caloric intake, weight gain and adiposity, ultimately producing a phenotype reminiscent of obesity. The data presented and discussed in the preceding chapters have demonstrated that ghrelin signalling, particularly central ghrelin signalling, is responsible for mediating some of these metabolic changes. However, ghrelin's biological activity and therefore its ability to influence metabolism upon release is entirely dependent on the post-translation modification by ghrelin- $O$-acyltransferase (GOAT). Given this, we hypothesized that pharmacological blockade of GOAT, leading to decreased circulating acylated ghrelin levels, would circumvent the development of stress-induced obesity in the face of chronic social defeat stress. To this end, we exposed mice to the chronic social defeat stress while administering a novel ghrelin-O-acyltransferase inhibitor daily. 


\subsection{Introduction}

Modern treatments of obesity have adopted an appetite-supressing mode of action to aid individuals with their dieting habits. However, it is now becoming more evident that appetite irregularities may not be the driving force behind the development of obesity, especially the development of stress induced obesity. It is now becoming more clear that hormonal influences can dictate an individual's phenotype and therefore play a prominent role in the development of obesity. Ghrelin is one of several hormones released in response to stress and is actively involved in the regulation of food intake and energy homeostasis. As previously discussed, ghrelin is the only known peripherally circulating hormone that has an appetite promoting effect and due to its role in the physiological response to stress, it has been suggested that ghrelin participates in the development of stress-induced obesity (Tschöp et al., 2000; Guo et al., 2007; Davies et al., 2009; Schellekens et al., 2012, 2013; Lutter and Sakata, 2008).

As discussed at length in Chapter 1, ghrelin contributes to long-term energy homeostasis by increasing body weight and adiposity through the reduction of lipid oxidation (Choi et al., 2003; Theander-Carrillo et al., 2006) and by increasing motivation to obtain foods that are highly palatable (Perello et al., 2010; King et al., 2011; Merkestein et al., 2012). The ability of ghrelin to carry out its physiological processes relies on its affinity to the growth hormone secretagogue receptor-1a (GHSR-1a), the only known ghrelin receptor. GHSR1a is widely expressed in both rodents and humans, with highest expression found in the hypothalamus and pituitary gland, both areas heavily invested in energy homeostasis and growth hormone secretion (Howard et al., 1996). Interestingly, activation of GHSR-1a requires a post-translation modification of the mature ghrelin protein (Kojima et al., 1999). The enzyme responsible for the acylation of the mature ghrelin protein is called ghrelin- $O$-acyltransferase, or GOAT (Gutierrez et al., 2008; Yang et al., 2008b). GOAT expression has been demonstrated in many rat, mouse and human tissues, including an exclusive distribution in ghrelin producing X/A-like cells of the gastric oxyntic mucosa lining the stomach (Sakata et al., 2009; Stengel et al., 2010; Lim et al., 2011) and it is believed that GOAT's only acylation target is ghrelin. The importance of GOAT in mediating the physiological role of ghrelin is highlighted by the fact that GOAT null mice have virtually no circulating plasma acylated ghrelin, and therefore no ligand-dependent activation of GHSR-1a (Gutierrez et al., 2008).

Given that stress induced obesity has become a world-wide health concern and that ghrelin is thought to mediate some of the physiological processes underlying the link between stress and obesity, we hypothesized that blunting the acylation of circulating des-acyl-ghrelin in response to stress would prevent the development of the metabolic dysfunctions that arise as a consequence of chronic stress. As such, we exposed mice to a chronic social defeat stress paradigm known to elicit increases in ghrelin secretion, and subsequent metabolic dysfunctions. To test our hypothesis, we administered daily injections of a novel putative GOAT antagonist, CF801, to animals exposed to the chronic social defeat stress paradigm. Results demonstrate that CF801 successfully decreased 
circulating plasma acylated ghrelin levels in mice. Furthermore, we demonstrate that mice exposed to CF801 showed a significant reduction in high-fat diet intake and overall caloric intake in response to the chronic social defeat stress paradigm compared to animals receiving vehicle injections.

\subsection{Materials \& Methods}

\subsubsection{Animals}

Male C57BL/6J mice ( $\mathrm{N}=52$ ), obtained from Jackson Laboratory (Bar Harbor, Maine) weighing 20-22g were used as experimental subjected. Retired breeding CD-1 male mice, aged 13-15 weeks and weighing 40-50g obtained from Charles River Farms (St Constant, Quebec, Canada) were used as bullies for the chronic social defeat stress paradigm. Mice were divided into 6 experimental groups and balanced such that each group had statistically equivalent body weights at the onset of the experiment. The experimental groups were as follows: non-injected controls non-stressed (NIC NS; $\mathrm{n}=10$ ), non-injected controls stressed (NIC $\mathrm{S} ; \mathrm{n}=10$ ), vehicle non-stress (VEH NS; $\mathrm{n}=8$ ), vehicle stress (VEH $\mathrm{S} ; \mathrm{n}=$ 8), CF801 non-stressed (CF801 NS; $\mathrm{n}=8$ ) and CF801 stressed (CF801 S; $\mathrm{n}=$ 8). Throughout the duration of the study, mice were housed under standard laboratory conditions and received ad libitum access to standard laboratory mouse chow and tap water as well as a daily 4-hour access to a high-fat diet containing 60\% caloric content from fat (TD 06414; Harlan Teklad, Indianapolis, Indiana) to measure intake of a preferred diet. High fat diet availability occurred from 10:00AM - 2:00PM daily. All procedures were approved by the Carleton University Animal Care Committee and followed the guidelines of the Canadian Council on Animal Care.

\subsubsection{CF801 Injections}

Throughout the baseline period, animals received no drug treatment. At the onset of the 21-day chronic social defeat stress paradigm, mice were given daily intraperitoneal (IP) injections of either vehicle ( $0.9 \%$ saline) or the putative ghrelin-O-acyltransferase inhibitor CF801 $(22 \mu \mathrm{mol} / \mathrm{kg}$; patent pending). Dosage was chosen based on preliminary data obtained from the provisional United States patent application (United States Patent Application Number: $61 / 857,879$ ) wherein $22 \mu \mathrm{mol} / \mathrm{kg}$ was sufficient in reducing acylated ghrelin, body weight, high-fat diet intake and and blunting a fast-induced refeeding bout in mice. Injections were delivered via a $27^{1 / 2}$ gauge stainless steel needle and were given immediately before the presentation of the high fat diet (10:00AM) each day. To prepare CF801, a $7.895 \mathrm{mg} / \mathrm{mL}$ solution was obtained by dissolving $12.6 \mathrm{~mL}$ of $0.9 \%$ saline into $50 \mathrm{mg}$ of CF 801 . This concentration was chosen in anticipation that each animal would receive an IP injection of approximately $0.2 \mathrm{~mL}$ per day and the solutions were prepared fresh each day prior to injections. 


\subsubsection{Chronic Social Defeat Stress Paradigm}

Following the two week baseline period, half of the mice in each group (NIC, VEH \& CF801) were removed from their room and transported to another room where they were each housed in a cage inhabited by a much larger sexually experienced male CD-1 mouse for the next 21 days. The stressed mice were protected from the aggressive CD-1 mice by an acrylic divider and wire mesh, yet olfactory, visual and auditory contact was maintained with the resident for the entire 21 day stress period. Each day the divider was removed and the animals were permitted to interact until the experimental mouse was subdued or until 15 minutes had passed. Measurements of regular chow intake, high fat diet intake, and body weight were recorded each day during the stress period.

\subsubsection{Behavioural Analysis}

\section{Elevated Plus Maze}

All animals were subjected to an elevated plus maze to measure anxiety levels. Briefly, mice were placed in the centre of the elevated plus maze apparatus and were permitted to explore the maze for 5 minutes. Mice were videotaped throughout the entire 5 minute period and the time spent in the open and closed arms of the maze, as well as the number of entries into the open arms of the maze were recorded by the experimenter.

\subsubsection{Hormone Analysis}

Following rapid decapitation, trunk blood was collected in EDTA-coated tubes placed on ice and centrifuged at $3000 \times \mathrm{g}$ for 15 minutes to separate plasma from red blood cells. Blood plasma was aliquoted separately for each assay to avoid multiple freeze/thaw cycles, and stored at $-80^{\circ} \mathrm{C}$ until processed. To protect the acylated ghrelin molecule, a $50 \mu \mathrm{l}$ aliquot of blood plasma was treated with $2.7 \mu \mathrm{l}$ of $1.0 \mathrm{~N}$ hydrochloric acid and $10 \mu \mathrm{l}$ of $100 \mathrm{mM} 4$-(hydroxymercuri) benzoic acid before storage. Plasma corticosterone levels were measured in duplicates using a commercially available RIA kit (ICN Biomedicals, Inc, Aliso Viejo, California). Plasma acylated ghrelin levels were measured using an ELISA kit (Millipore). All samples had a coefficient of variation $\leq 10$ and $15 \%$, respectively.

\subsubsection{Statistics}

All data were analyzed using $2 \times 3$ ANOVAs with drug (NIC vs. VEH vs. CF801) and treatment (stress vs. non-stress) as the between group factors, unless otherwise stated. All analyses were preceded with a Leven's Test of Quality of Error Variance to ensure the homogeneity of variance assumption was satisfied, and followed up with a LSD post-hoc analysis where necessary. The limit for statistical significance was set at $\alpha=0.05$. 


\subsection{Results}

\subsubsection{CF801 Reduces High Fat Diet Intake \& Overall Caloric Intake}

Regular chow consumption did not differ between any groups at any point during the baseline period (data not shown). Following the stress period, there was an expected increase in average daily regular chow consumption in stressed animals compared to their non-stressed controls (main effect of stress: $\mathrm{F}_{(5,40)}=38.361$, $p<0.001$; Figure 6.2A). Therefore, stressed animals, regardless of their drug group, consumed significantly more regular chow compared to their non-stressed controls. Furthermore, there was a significant effect of drug treatment on daily regular chow consumption (main effect of drug: $\mathrm{F}_{(5,40)}=3.531, p<0.05$ ). LSD post-hoc analysis revealed that animals receiving CF801, in general, consumed significantly more standard chow during the stress period compared to animals receiving vehicle injections (Figure 6.2D). Similarly, there was a significant drug and treatment effect on the change of regular chow consumption from the baseline to the stress period (main effects of drug and treatment: $\mathrm{F}_{(5,40)}=4.658, p$ $<0.05 \& \mathrm{~F}_{(5,40)}=40.865, p<0.001$, respectively). Therefore, stressed animals significantly increased the amount of regular chow consumed during the stress period, compared to what they were eating during the baseline period, relative to their non-stressed controls (Figure 6.2B). Furthermore, LSD post-hoc analysis demonstrated that animals receiving CF801, in general, did not decrease regular chow consumption during the stress period relative to both vehicle injected animals and non-injected controls (Figure 6.2E).

The average amount of high-fat diet consumed daily throughout the baseline period did not differ between groups (data not shown). During the stress period, however, there was a significant effect of treatment on daily high-fat diet intake, where stressed animals showed a significant reduction in high-fat diet intake (main effect of treatment: $\mathrm{F}_{(5,40)}=14.301, p<0.005$; Figure 6.3A). Furthermore, there was a significant effect of drug on the amount of daily high-fat diet consumed during the stress period (main effect of drug: $\mathrm{F}_{(5,45)}=19.295, p<$ 0.0001; Figure 6.3D), however no drug-treatment interaction was observed $(p$ $>0.05)$. An LSD post-hoc analysis revealed that animals receiving CF801, in general, consumed significantly less high-fat diet compared to animals receiving vehicle injections and non-injected controls $(p<0.05$; Figure 6.3D). Next, we analyzed the change in high-fat diet consumption from the end of the baseline period to the end of the stress period. Stress caused a significant reduction in the change in high-fat diet consumption (main effect of stress: $\mathrm{F}_{(5,40)}=7.144, p$ $<0.05$; Figure 6.3B). Similarly, drug treatment also caused a significant reduction in the change of high-fat diet consumption from the baseline to the stress period (main effect of drug: $\mathrm{F}_{(5,40)}=18.174, p<0.0001$; Figure 6.3E). LSD post-hoc analysis demonstrated that animals receiving CF801 injections, both stress and non-stressed, showed a significant reduction in the amount of high-fat diet consumed from the baseline period to the stress period $(p<0.05$; Figure 
$6.3 \mathrm{E})$.

The summed caloric content of both regular laboratory chow and the preferred high-fat diet was used to calculate the overall caloric consumption for each animal throughout the study. During the baseline, there were no differences in caloric consumption between any groups at any time point (data not shown). During the stress period there was a significant effect of stress on caloric consumption (main effect of stress: $\mathrm{F}_{(5,40)}=25.859, p<0.0001$; Figure $6.4 \mathrm{~A}$ ), where stressed animals consumed significantly more calories then their non-stressed controls across all levels of drug treatment (Figure 6.4A). Furthermore, there was a significant effect of drug treatment on caloric consumption during the stress period (main effect of drug: $\mathrm{F}_{(5,40)}=13.009, p<0.0001$ ). Post-hoc analysis demonstrated that animals receiving CF801 treatment, in general, consumed significantly less calories during the stress period compared to non-injected control animals. Stressed animals receiving CF801 treatment consumed significantly less calories compared to non-injected control stressed animals and vehicle injected stress animals $(p$ 's $<0.05)$, suggesting that CF801 decreases overall caloric intake in response to a chronic stressor (Figure 6.4A). There was a significant interaction between drug treatment and stress on the change in caloric intake from the baseline to the stress period (significant drug $\times$ treatment interaction: $\mathrm{F}_{(5,40)}=3.559, p<0.05$; Figure 6.3B). Figure 6.4B illustrates that non-stressed animals receiving CF801 consume significantly less calories compared to non-injected and vehicle injected non-stressed animals, demonstrating CF801's ability to reduce overall caloric intake. Furthermore, the influence of stress on changing caloric intake is dependent on the presence or absence of CF801. Therefore, stressed animals receiving CF801 show significant reduction in overall caloric intake in response to the stressor relative to stressed animals receiving no injections or vehicle injections $(p$ 's $<0.05$; Figure $6.4 \mathrm{~B})$.

\subsubsection{CF801 Causes a Reduction in Body Weight in both Stressed \& Non-Stressed Animals}

There were no group differences at any point during the baseline period on body weight (data not shown). Following the stress period, however, there was a significant drug effect on body weight $\left(\mathrm{F}_{(5,45)}=14.041, p<.05\right.$; Figure $\left.6.1 \mathrm{~A}\right)$. An LSD post-hoc analysis revealed that animals receiving CF801 (both stressed and non-stressed animals) weighed significantly less compared to non-injected control animals. Animals receiving vehicle injections, however, showed no differences in body weight compared to animals receiving CF801 and/or non-injected controls $(p>0.05)$. We next measured the change in body weight from the end of baseline to the end of the stress period. There was a significant treatment effect on the change in body weight (main effect of stress: $\mathrm{F}_{(5,40)}=4.340, p$ $<0.05)$, where stress caused a significant reduction in body weight gain when groups were collapsed across all levels of drug treatment (Figure 6.1B). Although there was no significant interaction between drug and treatment on the change in body weight, it appeared as if the significant reduction in body weight in 
stressed animals was coming from animals in the vehicle injected group. Therefore, an LSD post-hoc analysis was performed to investigate if stressed animals in all groups were showing a reduction in body weight, or if just animals in the vehicle group were showing this trend. Post-hoc analysis demonstrated that vehicle injected stressed animals gained significantly less body weight compared to their non-stressed controls $(p<0.05)$, however the differences in body weight change between non-injected control stressed and non-stressed animals, as well as the change in body weight between CF801 stressed and non-stressed animals did not attain statistical significant ( $p$ 's $=0.115 \& 0.612$, respectively). Furthermore, there was a significant drug effect on the change in body weight (main effect of drug $\mathrm{F}_{(5,40)}=28.293, p<0.001$; Figure 6.1D). Post hoc analysis revealed that animals receiving both vehicle and CF801 injections gained significantly less body weight compared to non-injected control animals. Moreover, animals receiving CF801 treatment gained significantly less body weight compared to vehicle injected animals, suggesting that CF801, in general, was capable of reducing body weight.

\subsubsection{Stress Tended to Increase Blood Glucose Indepen- dently of CF801 Treatment}

Following rapid decapitation, samples of trunk blood were collected and processed for hormonal profiles. Figure 6.5A,B illustrate circulating blood glucose at the time of sacrifice. Here, we show a tendency for stress animals, regardless of their drug treatment, to have higher levels of circulating blood glucose compared to non-stressed animals (main effect of stress: $\mathrm{F}_{(5,36)}=3.530, p=$ 0.068). However, we did not observe a significant effect of drug treatment, nor a drug $\times$ treatment interaction on blood glucose levels.

\subsubsection{Stress Increases Circulating Plasma Acylated Ghre- lin, While CF801 Treatment Blunts This Effect}

It has been previously shown, through work done in the current thesis and by others, that plasma acylated ghrelin increases in response to stressors. Here, we confirm these findings by demonstrating that stressed animals have significantly more acylated ghrelin levels compared to non-stressed animals (main effect of stress: $\mathrm{F}_{(5,36)}=31.481, p<0.0001$; Figure $6.5 \mathrm{C}$ ). Interestingly, there was a significant drug effect (main effect of drug treatment: $F_{(5,36)}=18.669$, $p<0.0001$ ) where animals receiving CF801 treatment had significantly lower acylated ghrelin levels compared to non-injected and vehicle injected animals (Figure 6.5D). There was also a significant drug $\times$ stress interaction (significant drug $\times$ stress interaction $\left(\mathrm{F}_{(5,36)}=4.483, p<0.05\right)$. LSD post-hoc analysis revealed that while stress was capable of eliciting a significant increase in acylated ghrelin in non-injected and vehicle injected animals $(p$ 's $<0.05)$, CF801 treatment abolished the increase in acylated ghrelin seen in stressed animals $(p$ $>0.05$; Figure $6.4 \mathrm{C}$ ). 


\subsubsection{Stress Increases Anxiety-like Behaviours}

\section{Elevated Plus Maze}

There was a significant effect of treatment on the animals performance on the elevated plus maze. Stressed animals, regardless of the drug treatment, spent significantly less time in the open arms of the elevated plus maze (main effect of stress: $\mathrm{F}_{(5,40)}=9.782, p<0.005$; Figure $6.6 \mathrm{~A}$ ). While stress was successful in reducing the amount of time spent in the open arm of the maze, there was no effect of drug treatment (Figure 6.6C). Interestingly, there was a significant drug effect on the number of entries each animal made into the open arms of the maze (main effect of drug: $\mathrm{F}_{(5,40)}=4.288, p=0.021$ ). An LSD post-hoc analysis demonstrated that animals receiving CF801 (both stressed and nonstressed) entered the open arms of the elevated plus maze significantly less then animals receiving vehicle injections $(p<0.05$; Figure $6.6 \mathrm{D})$. However, there was no difference in the number of entries into the open arm made between non-injected control animals and animals receiving CF801 injections.

\subsection{Discussion}

Stress-induced obesity has long been associated with increases in caloric intake and subsequent storage of body mass in the form of adipose tissue. More recently, it has been demonstrated that metabolic hormones, such as ghrelin, released in response to stress contribute to the development of metabolic disorders such as obesity. Work from this thesis suggests that ghrelin signalling in response to stress elicits an increase in overall caloric intake and the promotion of carbohydrate utilization, ultimately producing an obese-like phenotype in mice. The present experiment was aimed at investigating the metabolic outcome of a chronic stressor in the absence of ghrelin signalling through GOAT inhibition. To address this, we exposed mice to a chronic social defeat stress paradigm previously shown to cause an increase in caloric intake and the development of an obese-like phenotype, while administering a novel GOAT antagonist CF801 (patent pending). GOAT is the only known enzyme capable of acylating, and therefore activating, the mature ghrelin protein and GOAT null mice show a complete absence of circulating acylated ghrelin (Gutierrez et al., 2008). Therefore, GOAT antagonists have recently become a target for the development of anti-obesity treatments.

Results from this study confirm that the chronic social defeat stress elicited a significant increase in caloric intake, an effect resultant from increases in regular chow consumption and not the preferred high fat diet. Therefore the chronic social defeat stress, as shown in previous chapters, caused a significant increase in regular chow intake and a significant reduction in high-fat diet intake. Interestingly, CF801 had similar effects on the pattern of caloric intake as did the chronic stress paradigm. As illustrated in Figures 6.2, non-stressed animals receiving no injections or vehicle injections showed a significant reduction in regular chow consumption as they became more familiar with the highly palatable, 
high fat diet. However, non-stressed CF801 treated animals continued to consume equivalent amounts of regular chow as they did during the baseline period. Moreover, stress prevented animals from increasing the amount of high-fat diet consumed and, as a result, non-stressed animals consumed significantly more high-fat diet then their stressed counterparts. Similarly, CF801 treated animals showed a significant reduction in high-fat diet consumption while non-injected and vehicle injected animals continued to consume high-fat diet during the stress period. These results demonstrate that CF801 is capable of reducing the intake of a high-fat diet which is in agreement with others who have demonstrated that GOAT-null mice reduce hedonic feeding and that interruption of GOAT activity leads to an overall decrease in caloric intake (Davis et al., 2012; Teubner et al., 2013). What is unknown, however, is why CF801 treatment seems to prevent animals from discontinuing regular chow consumption. There are several possible mechanisms at play. First, despite decreasing the amount of high-fat diet consumed, animals treated with CF801 must consume their daily quota of calories in order to maintain a homeostatic balance. It is possible that animals will choose their regular laboratory chow over the high-fat diet in the presence of CF801, therefore suggesting CF801 can alter the dietary preference in these animals. In contrast, stress promotes an increase in regular chow consumption presumably to ensure the availability of a rapid fuel source, rather then altering dietary preferences. Therefore, while both stress and CF801 result in an increase in regular chow and a decrease in high-fat diet, their mechanisms may be very different. This is important to note because although CF801 produces the same patterns in caloric intake as does chronic social stressors, CF801 does not lead to an overall increase in caloric intake whereas the stressor does. Another possibility is that stress, CF801, and/or both, induce an anhedonic state in animals. It is known that animals who lack an intact ghrelin signalling system are more likely to develop depressive like symptoms, including anhedonia (Lutter and Sakata, 2008). It is possible, however currently unknown, that CF801 injections produce an anhedonic state in these animals. More experiments are required to answer this question.

Chronic social defeat stress caused a significant increase in plasma acylated ghrelin in non-injected control animals, as well as vehicle injected animals. Interestingly, this increase in plasma acylated ghrelin did not occur in stressed animals receiving CF801 injections. This is the first demonstration that the a putative GOAT antagonist is capable of preventing stress induced ghrelin secretion. Importantly, there was still evidence of circulating acylated ghrelin in animals receiving CF801 injections. This suggest that injections of CF801 do not block $100 \%$ of ghrelin acylation, but rather it reduces the ability of GOAT to acylate ghrelin in times of high-ghrelin output (i.e. stress). Moreover, the pattern of caloric intake seen in stressed animals fits very nicely with the aforementioned pattern of ghrelin secretion. Non-injected and vehicle injected stressed animals both show increases in circulating plasma acylated ghrelin as well as increases in overall caloric intake during the stress period, results that are in agreement with the known physiological role of ghrelin (Tschöp et al., 2000; Choi et al., 2003; Theander-Carrillo et al., 2006; Guo et al., 2007; Davies 
et al., 2009; Lutter and Sakata, 2008) . In contrast, stressed animals receiving CF801 do not show an increase in plasma acylated ghrelin and do not show increases in caloric intake in response to the stressor, suggesting that CF801 prevents stress-induced increases in caloric intake. These are powerful data to support the use of GOAT inhibitors for the treatment of stress induced increases in caloric intake.

It has long been understood that stress-induced caloric intake is associated with increases in body weight, particularly from increases in adipose tissue (Rowland and Antelman, 1976; Foster and Solomon, 2006; Solomon et al., 2007; Kuo et al., 2007). Previous work from this thesis is in agreement with these findings, where chronic social defeat stress was shown to increase caloric intake, body weight and adiposity in mice. While the body composition of animals from this study were not analyzed, there was no significant increases in body weight as a consequence of stress. In fact, the vehicle injected stressed animals lost a significant amount of body weight during the stress period compared to their non stress controls. The absence of weight gain in stressed animals, despite an increase in circulating ghrelin and subsequent caloric intake throughout the entire 21 day stress paradigm is not fully understood. It is known that age is an important variable in ghrelin-mediated increases in body weight (Lin et al., 2011), and it is therefore possible that these animals were resistant to changes in body weight due to a scarcity in GHSR-1a expression. However this is unlikely given that other work from this thesis describes animals of roughly the same age who have shown ghrelin-dependent changes in body weight. Despite stressed animals not showing the anticipated increase in body weight, their metabolic profile (i.e. increased acylated ghrelin, increased caloric intake, etc.) suggests that, over time, these animals are at risk for increased body weight and development of obesity. Animals treated with CF801, however, do not share this metabolic profile and would likely be spared from these metabolic consequences down the road.

In addition to ghrelins role in the development of stress-induced metabolic changes, it has been shown that ghrelin mediates the behavioural consequences of stress (Lutter and Sakata, 2008; Spencer et al., 2012). The anxiolytic properties of ghrelin remain to be fully understood, as there are still laboratories who suggest that ghrelin has anxiogenic effects (Currie et al., 2012; Carvajal et al., 2009). Here we show that stress produces some anxiety-like behaviours, evidenced by stressed animals spending significantly less time in the open arms of the elevated plus maze. These results are in agreement with other labs who demonstrate that social stress causes anxiety-like behaviours in an elevated plus maze (Berton et al., 1998; Ruis et al., 1999; Krishnan et al., 2007; McCormick et al., 2008). CF801 did not influence the anxiogenic effects of the stressor one way or another. However, mice receiving CF801 injections did show a significant reduction in the number of entries made into the open arms of the elevated plus maze relative to vehicle injected animals. Although not conclusive, these data suggest that CF801 has anxiogenic potential, and blunting ghrelin secretion may precipitate anxiety in mice. More work needs to be done to better characterize the contribution of ghrelin to behaviours in response to chronic stress. Previous 
work from our laboratory has failed to see differences in anxiety-like behaviours in GHSR-KO animals (data not shown), thus there remains some uncertainty as to what effects ghrelin has on anxiety.

Overall, these data contribute to the growing body of literature suggesting that ghrelin contributes to the development of metabolic disorders by promoting caloric intake in response to stressors. Furthermore, these data provide a very attractive option for blunting stress-induced ghrelin secretion and subsequent increases in overall caloric intake through the use of GOAT inhibitors. The putative GOAT inhibitor CF801 has sufficiently shown evidence for its ability to inhibit GOAT activity. Reductions in circulating acylated ghrelin, decreases in body weight and high-fat diet consumption are all in support of an efficient GOAT inhibitor. There are several limitations to this study. First, CF801 has not been shown experimentally to inhibit GOAT activity. Therefore, experimental evidence demonstrating that CF801 can successfully block, or reduce, GOAT activity will be required before any concrete conclusions can be drawn. However, these data suggest that reducing acylated ghrelin has beneficial effects regardless of the mechanisms of CF801 action. Secondly, these data show that reducing acylated ghrelin levels through GOAT inhibition is beneficial for the prevention of stress-induced metabolic dysfunctions, however they do not indicate what the outcome may be if an organism already had a metabolic dysfunction. As is usually the case in clinical settings, patients would seek help after the metabolic changes have already occurred. More studies are required to investigate the role of CF801 in reversing, or ameliorating pre-existing metabolic dysfunctions. 


\subsection{Figures \& Tables}
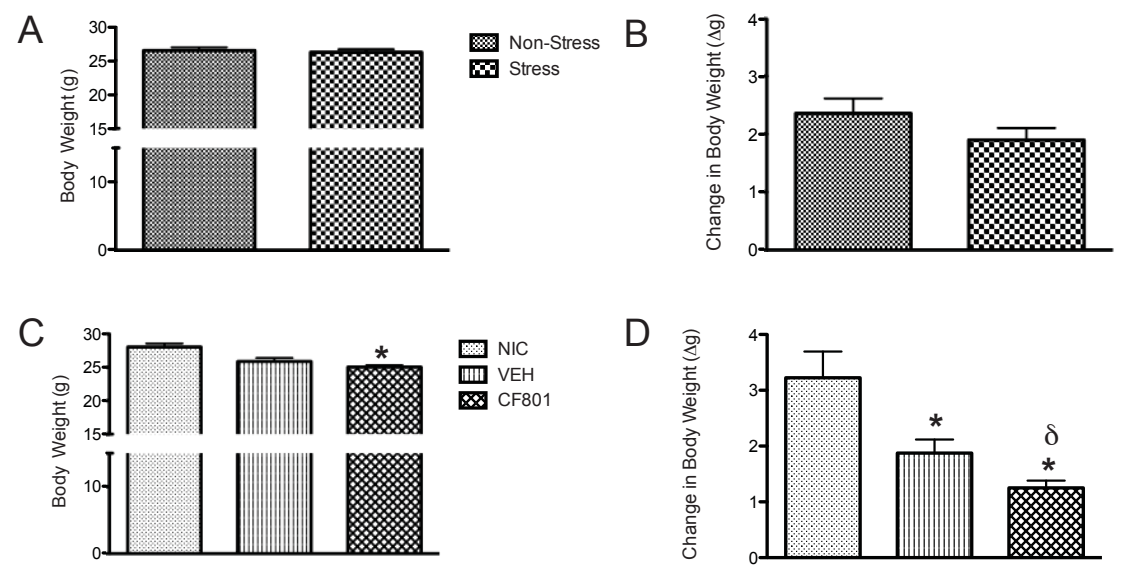

Figure 6.1: Average body weight during the stress period (A \& C); change in body weight from the end of the baseline to the end of the stress period (B \& D). ${ }^{*} p<0.05$ relative to non-injected control mice; ${ }^{\delta} p<0.05$ relative to vehicle injected controls. NIC, non injected control; VEH, vehicle. 

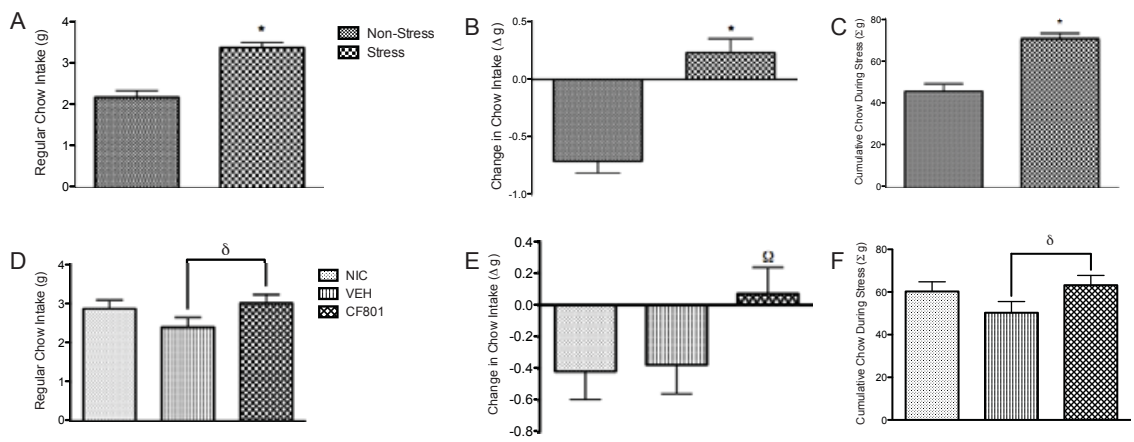

Figure 6.2: Average daily regular chow consumption during the stress period (A $\&$ D); change in regular chow consumption from the end of baseline to the end of the stress period (B \& E); cumulative regular chow intake during the stress period $(\mathrm{C} \& \mathrm{~F}) ;{ }^{*} p<0.05$ relative to non-stressed controls; ${ }^{\delta} p<0.05$ relative to vehicle injected controls; ${ }^{\Omega} p<0.05$ relative to vehicle and non-injected controls. NIC, non injected control; VEH, vehicle. 

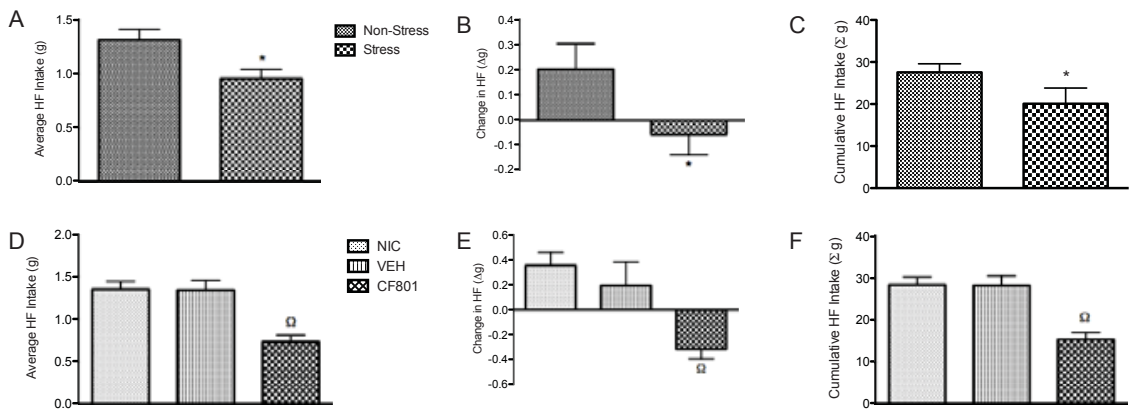

Figure 6.3: Average daily high fat diet consumption during the stress period (A $\&$ D); change in high-fat diet consumption from the end of baseline to the end of the stress period $(\mathrm{B} \& \mathrm{E})$; cumulative high fat diet intake during the stress period $(\mathrm{C} \& \mathrm{~F}) ;{ }^{*} p<0.05$ relative to non-stressed controls; ${ }^{\Omega} p<0.05$ relative to vehicle and non-injected controls. NIC, non injected control; VEH, vehicle. 

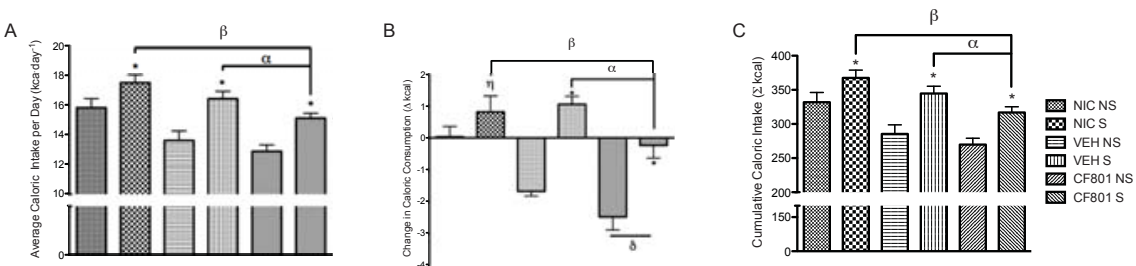

Figure 6.4: (A) Average daily caloric intake during the stress period; (B) change in caloric consumption from the end of baseline to the end of the stress period; (C) Cumulative caloric intake during the stress period; ${ }^{*} p<0.05$ relative to non-stressed controls; ${ }^{\eta} p<0.10$ relative to non-stressed controls; ${ }^{\alpha} p<0.05$ relative to vehicle injected stressed animals. ${ }^{\beta} p<0.05$ relative to non-injected control stressed animals. ${ }^{\delta} p<0.05$ relative to non-injected and vehicle injected controls; NIC, non injected control; VEH, vehicle. 

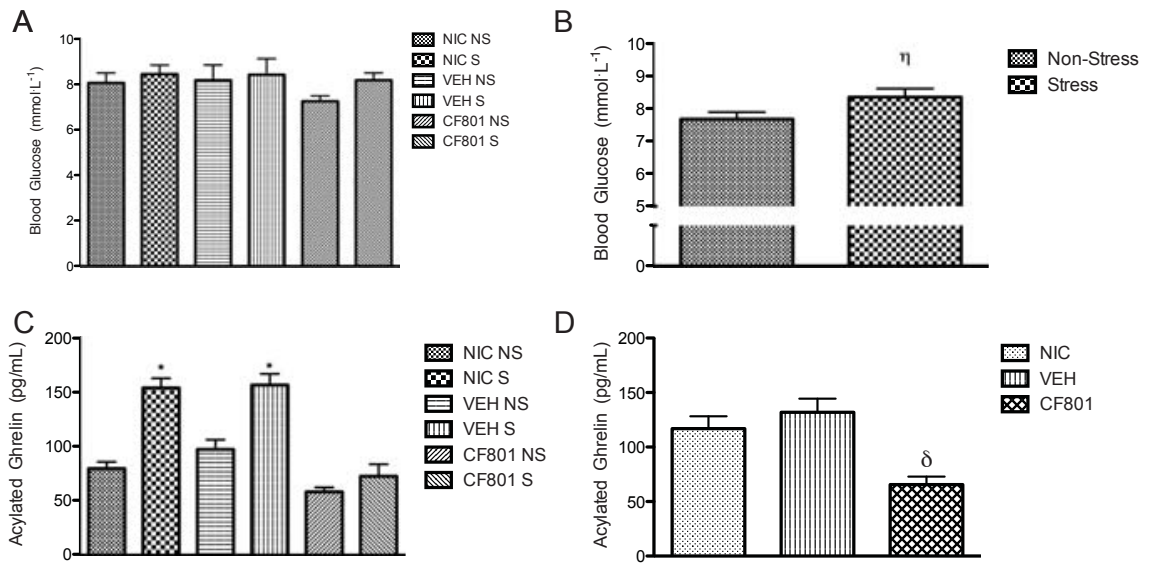

Figure 6.5: Concentration of blood glucose (A \& B) and acylated ghrelin (C $\&$ D) in trunk blood collected at the time of sacrifice, 24 hours after their last stressor. ${ }^{*} p<0.05$ relative to non-stressed controls. ${ }^{n} p<0.10$ relative to non-stressed controls; ${ }^{\delta} p<0.05$ relative to non-injected and vehicle injected controls. NIC, non-injected control; VEH, vehicle. 

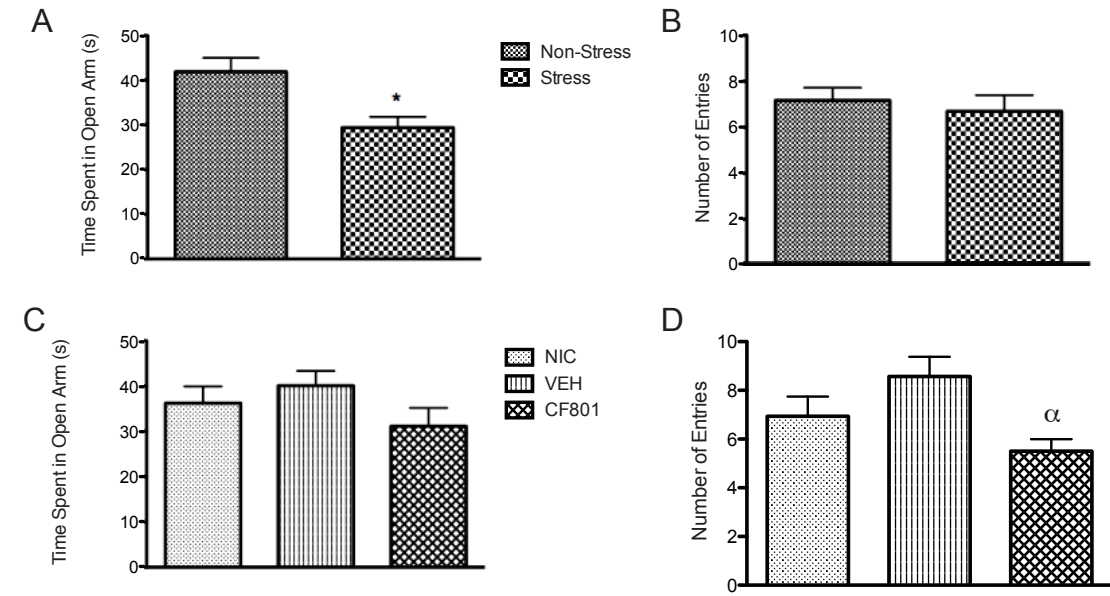

Figure 6.6: Time spent in the open arm of the elevated plus maze (A \& C); number of entries into the open arm of the elevated plus maze (B \& D). ${ }^{*} p$ $<0.05$ relative to non-stressed controls; ${ }^{\alpha} p<0.05$ relative to vehicle injected animals; NIC, non-injected control; VEH, vehicle. 


\section{Chapter 7}

\section{General Discussion}

The present thesis set out to examine and describe the role of ghrelin in mediating the metabolic changes that occur as a consequence of chronic stress. The experiments conducted and described throughout Chapters 2-6 provide some fundamental answers that address the role of ghrelin in mediating stress-induced physiological changes, and investigates potential therapeutic treatments. As a whole, this body of work suggests that ghrelin is secreted in response to stress which represents an organisms attempt at strategically mobilizing a rapid energy source which serves as fuel for the organism to deal with the stressor at hand. Concurrently, the stress-induced ghrelin secretion promotes caloric intake, presumably to replenish an organisms energy reserve in the face of chronic stress. These findings place ghrelin atop a list of hormones necessary for an appropriate physiological response to stress. Like glucocorticoids, for example, ghrelin is required to produce a normal response to stress and in the absence of ghrelin signalling an organism cannot suitably respond to a stressor, both physiologically and/or psychologically speaking. It is possible that ghrelin is as much of a stress hormone as glucocorticoids, the traditional culprit of stressinduced pathologies. To support this claim, it has been shown that ghrelin levels rise in response to stressors, but remain elevated for an extended period of time following cessation of the stressor (Lutter and Sakata, 2008). Therefore, while glucocorticoid secretion may subside rapidly upon removal of a stressor, ghrelin levels linger and thus provide a longer lasting marker of stress exposure. Similarly, it has been shown that stress-induced emotional eating in humans is a function of ghrelin, and not glucocorticoid, signalling (Raspopow et al., 2010). Acutely, the metabolic influences exerted by ghrelin in response to stress are beneficial. Chronic stress, on the other hand, creates an environment conducive to the development of metabolic disorders, and the present thesis demonstrates that many of these are ghrelin dependent.

One of the most impressive findings from this work is that ghrelin appears to defend against the breakdown of adipocytes, and consequently promotes the breakdown of carbohydrates as a fuel source. This finding remains consistent throughout the entire body of work presented in this thesis. Admittedly, there 
are several distinct metabolic outcomes discussed that appear as a consequence of varying experimental protocols, however the fact that ghrelin signalling promotes the preservation of fat stores remains a common theme throughout. For example, Chapter 2 describes an experiment wherein WT mice show reductions in food intake and body weight, despite showing elevated circulating ghrelin levels. These data were thought to contradict the well known physiological role of ghrelin, however discrepancies were attributed to the lack of predictability of the stress paradigm. In contrast, Chapters 3 and 4 describe a metabolic state where animals increased their caloric intake and body weight in response to a very mild and predictable chronic social defeat stress. Despite the opposing effects on food intake and body weight between these two stress paradigms, the common denominator linking stress-induced ghrelin secretion to adiposity is the fact that both groups of animals show increases in visceral adipose tissue. Furthermore, Chapter 3 describes the phenotype of animals exposed to a chronic social defeat stress in the absence of ghrelin signalling (i.e. GHSR KO mice). Here, the absence of GHSR-1a prevented stress from increasing respiratory exchange ratio and therefore the promotion of carbohydrate utilization. These data provide further evidence that ghrelin is protecting adipose tissue as the GHSR KO stressed animals metabolized adipose tissue and showed a significant reduction of visceral adipose tissue as a consequence. Together, the data from the present thesis provides evidence that the conservation of fat stores is the overarching role of stress induced ghrelin secretion. It is unclear what the detailed body composition of animals from Chapter 6 is, however given their hormonal profiles one can hypothesize that stressed animals will show an increase in adiposity while animals who received CF801 administration will not.

One way in which ghrelin is capable of protecting adipose tissue is through the promotion of carbohydrate utilization. Chapter 3 demonstrates that ghrelin signalling increases respiratory exchange ratio's in stressed animals, ultimately promoting the breakdown of carbohydrates as a fuel source. These data are in agreement with others demonstrating that ghrelin increases respiratory quotients, and therefore promotes carbohydrate utilization (Tschöp et al., 2000; Currie et al., 2005). Overtime, the utilization of carbohydrates as a fuel source in the absence of any adipose tissue metabolism would lead to the accumulation of fat mass and development of obesity, a phenotype described by Depke et al. (2008). Furthermore, it has been suggested that ghrelin may promote adipogenesis through up-regulating PPAR $\gamma 2$ mRNA expression and ultimately the formation of new pre-adipocytes, however these effects have not been shown in vivo (Choi et al., 2003). Finally, it has been argued that ghrelin could promote adiposity, independently of food intake, through the expansion of mature adipocytes. Indeed, work from Chapter 3 corroborates these findings as animals with highest ghrelin levels showed evidence of adipocyte swelling. The exact mechanism by which ghrelin protects fat mass in the face of chronic stress remains to be fully understood. However, data presented in this thesis provides evidence that ghrelin is protecting adipose tissue in response to different stress paradigms with diverse metabolic outcomes.

It is important that an organism has access to a rapidly available energy sub- 
strate in times of stress. Therefore, it is possible that ghrelin provides a survival advantage by promoting the utilization of a rapid energy source. Thus, acutely, ghrelin secretion in response to stress is likely beneficial to the survival of the organism. However, prolonged stressors present a difficult metabolic challenge to the organism and could represent the point at which chronic stress becomes detrimental. If ghrelin is constitutively elevated over a long period of time, increased carbohydrate utilization and the conservation of fat mass may become detrimental to the organism (Depke et al., 2008). Data from the current thesis would suggest that 3 weeks of chronically elevated ghrelin is long enough to elicit detrimental changes to an organism, however the exact timeline highlighting the point at which ghrelin signalling becomes detrimental is not yet understood.

For example, how long are animals sensitive to increases in circulating acylated ghrelin? It has been shown that stress causes a significant increase in ghrelin, and that ghrelin concentrations remain elevated for extended period of time after the cessation of the stressor (Lutter and Sakata, 2008). Furthermore, we are presenting data of a three week chronic social defeat stress where ghrelin levels are presumably elevated throughout the entire paradigm. It is possible that ghrelin sensitivity is decreased at some point, however there lacks evidence exploring this idea. What is known, however, is that GHSR-1a internalization would be a mode of desensitization of an organism to ghrelin signalling. Furthermore, circulating fatty acids may in fact dictate the ease of which GHSR-1a becomes internalized. For example, Delhanty et al. (2010b) have shown that high levels of unsaturated fatty acids prevent the internalization of GHSR-1a, and therefore prevent desensitization of ghrelin treatment. In all experiments presented in this thesis animals had restricted access to a high-fat diet containing a large proportion of unsaturated fatty acids. Therefore, the presence of a high-fat diet might in fact prevent animals from desensitizing to the chronically elevated levels of ghrelin and thus prevent desensitization. However, the work showing fatty acid suppression of receptor internalization was in vitro, and therefore it is unclear how diet availability would influence receptor internalization in vivo. Again, more work is required to address this issue.

\subsection{Conclusion}

Together these data provide evidence that ghrelin participates in the physiological responses to stress and ghrelin may serve as a reliable marker for stress exposure. Central ghrelin signalling is important for the promotion of energy consumption that is required to deal with a chronic stressor. Ghrelin may be playing a protective role by promoting the utilization of carbohydrates as a rapid fuel source while sparing the breakdown of fat mass. Over time, this metabolic phenotype leads to increased body weight and adiposity, ultimately producing an obese like state in rodents. Interruption of ghrelin signalling, both genetically and pharmacologically, is capable of reducing some of the negative consequences associated with chronic stress. It is possible that ghrelin signalling influences the AMPK signalling cascade somewhere within the hypothalamus to adjust an 
organisms internal fuel gauge, and shift the homeostatic scales in favour of energy consumption and body weight gain. Furthermore, ghrelin may recruit the orexin neurons in the LH to stimulate food motivated behaviours and therefore promote food intake. Therapeutic avenues exploring potential treatments for stress-induced metabolic dysfunctions should consider targeting the ghrelin signalling system. Here we have proposed the use of a putative GOAT antagonist, CF801, as a potential therapeutic agent to treating stress-induced metabolic disorders. 
Chapter 8

\section{Appendix A - Supplemental Figures}




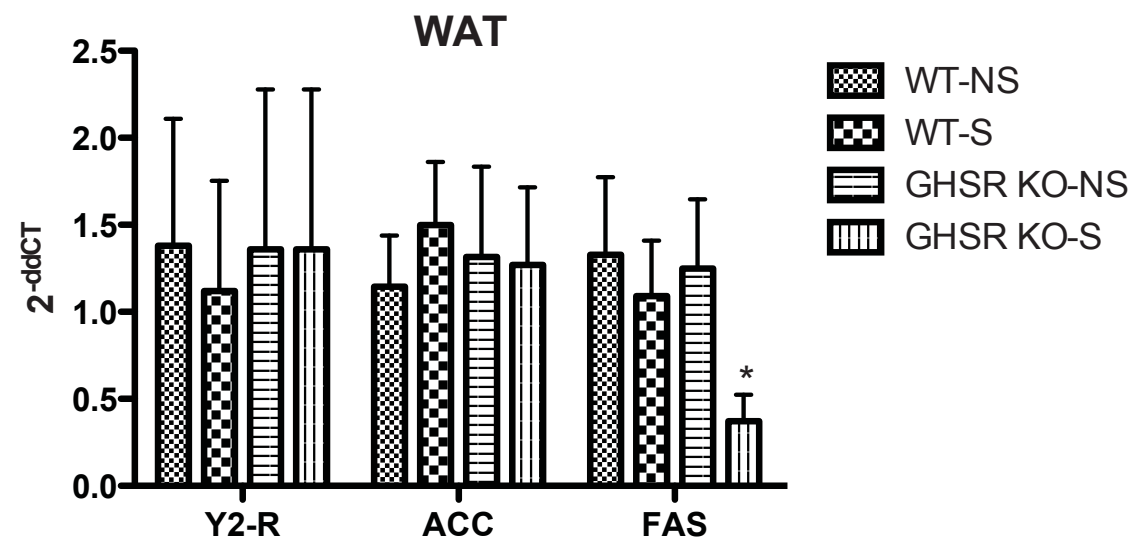

Figure 8.1: mRNA expression of Y2-R, acetyol-CoA carboxylase and fatty acid synthase in white adipose tissue. All values are expressed as mean $\pm \mathrm{SEM}$, relative to GAPDH expression. 
Chapter 9

\section{Appendix B - Supplemental Tables}




\begin{tabular}{lc}
\hline Primer & Sequence \\
\hline GAPDH-F & GCA GTG GCA AAG TGG AGA TTG TTG C \\
GAPDH-R & CCC GTT GAT GAC AAG CTT CCC ATT C \\
NPY-F & TAC CCC TCC AAG CCG GAC AA \\
NPY-R & TTT CAT TTC CCA TCA CCA CAT G \\
AgRP-F & CGG AGG TGC TAG ATC CAC AGA \\
AgRP-R & AGG ACT CGT GCA GCC TTA CAC \\
Y2R-F & AGA TCA TTC CTG ACT TTG AG \\
Y2R-R & GAA CAC GGT GAA GAT GAG \\
FAS-F & CAT GAC CTC GTG ATG AAC GTG T \\
FAS-R & CGG GTG AGG ACG TTT ACA AAG \\
ACC-F & ATT GGG CAC CCC AGA GCT A \\
ACC-R & CCC GCT CCT TCA ACT TGC T \\
\hline
\end{tabular}

Table 9.1: RT-qPCR Sequences 


\section{Chapter 10}

\section{Appendix C - Copyright Documentation}

\subsection{Permission from Frontiers in Neuroendocrin- iology}

The following has been extracted from the Frontiers website (http://www.frontiersin.org/about/faq)

- Under the Frontiers Terms and Conditions, authors retain the copyright to their work. All Frontiers articles are Open Access and distributed under the terms of the Creative Commons Attribution License, (CC-BY 3,0), which permits the use, distribution and reproduction of material from published articles, provided the original authors and source are credited, and subject to any copyright notices concerning any third-party content.

\subsection{Permission from European Journal of Neu- roscience}

Dear Mr. Zachary Patterson,

Thank you for placing your order through Copyright Clearance Center's RightsLink service. John Wiley and Sons has partnered with RightsLink to license its content. This notice is a confirmation that your order was successful.

Your order details and publisher terms and conditions are available by clicking the link below: http://s100.copyright.com/CustomerAdmin/PLF.jsp?ref=1f0d4367- 
b6e7-4c15-b116-ae33f56c9e8b

Order Details

Licensee: Zachary R Patterson

License Date: Jul 30, 2013

License Number: 3198790178116

Publication: European Journal of Neuroscience

Title: Altered metabolic and neurochemical responses to chronic unpredictable stressors in ghrelin receptor-deficient mice

Type Of Use: Dissertation/Thesis

Total: 0.00 USD

To access your account, please visit https://myaccount.copyright.com.

Please note: Online payments are charged immediately after order confirmation; invoices are issued daily and are payable immediately upon receipt.

To ensure we are continuously improving our services, please take a moment to complete our customer satisfaction survey.

B.1:v4.2

\subsection{Permission from Endocrinology}

1. Endocrinology

ISSN: $1945-7170$

Publication Type: e-Journal

Volume: 154

Issue: 3

Start page: 1080

Publisher: ENDOCRINE SOCIETY

Author/Editor: Endocrine Society; HighWire Press

Permission type:Republish or display content

Type of use:Republish in a thesis/dissertation

\subsection{Permission from Neuroscience}

Dear Mr. Zachary Patterson,

Thank you for placing your order through Copyright Clearance Center's RightsLink service. Elsevier has partnered with RightsLink to license its content. This notice is a confirmation that your order was successful.

Your order details and publisher terms and conditions are available by clicking the link below: http://s100.copyright.com/CustomerAdmin/PLF.jsp?ref=d95e5985- 
a1e2-40ab-8f3b-ce43ea0b5c5d

Order Details

Licensee: Zachary R Patterson

License Date: Jul 30, 2013

License Number: 3198790779369

Publication: Neuroscience

Title: Ghrelin-deficient mice have fewer orexin cells and reduced cFOS expression in the mesolimbic dopamine pathway under a restricted feeding paradigm Type Of Use: reuse in a thesis/dissertation Total: 0.00 USD

To access your account, please visit https://myaccount.copyright.com.

Please note: Online payments are charged immediately after order confirmation; invoices are issued daily and are payable immediately upon receipt.

To ensure we are continuously improving our services, please take a moment to complete our customer satisfaction survey.

B.1:v4.2

\subsection{Co-Author Permission Statements}


To whom it may concern,

I, Albert Isaacs, hereby grant Zachary Patterson permission to use all contents associated with the manuscript, Interruption of Ghrelin Signalling in the PVN Increases High Fat Diet Intake \& Body Weight in Male C57BL6J Mice published in Frontiers in Neuroendocrine Science, as part of his $\mathrm{PhD}$ dissertation.

Aytsere

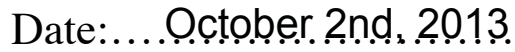


To whom it may concern,

I, Alfonso Abizaid, hereby grant Zachary Patterson permission to use all contents associated with the manuscript, Stress Induced Obesity: Lessons from Rodent Models of Stress published in Frontiers in Neuroendocrine Science, as part of his $\mathrm{PhD}$ dispertation.

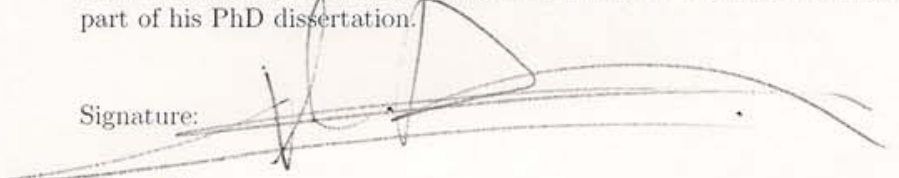

Date: $S_{\text {DP }} 23^{\text {AS }}, 2013$ 
To whom it may concern,

I, Alfonso Abizaid, hereby grant Zachary Patterson permission to use all contents associated with the manuscript, Ghrelin Deficient Mice Have Fewer Orexin. Cells $\&$ Reduced cFos Expression in the Mesolimbic Dopamine Pathway Under a Restricted Feeding Paradigympublished in-Neuroscience as part of his PhD dissertation.

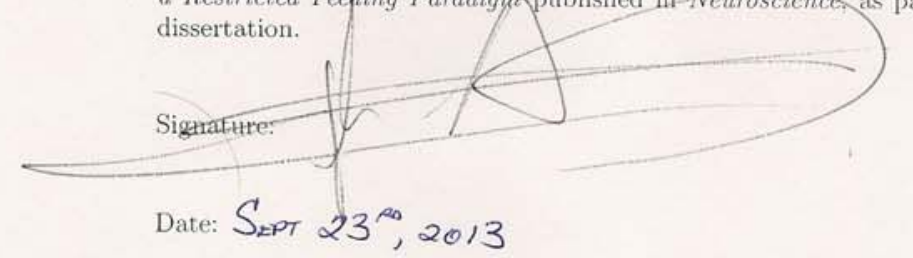


To whom it may concern,

I, Alfonso Abizaid, hereby grant Zachary Patterson permission to use all contents associated with the manuscript, Central Ghrelin Signalling Mediates the Metabolic Response of C57 BL6J Male Mice to Chronic Social Defeat Stress published in Endocrinology, as part of his $\mathrm{PhD}$ dissertation.

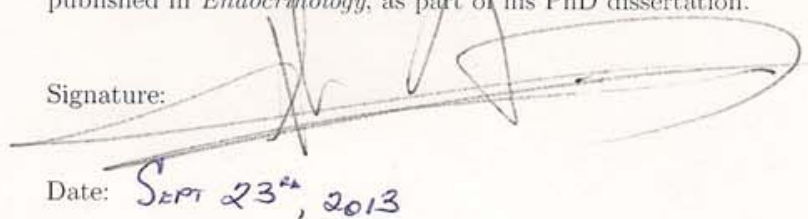


To whom it may concern,

I, Alfonso Abizaid, hereby grant Zachary Patterson permission to use all contents associated with the manuscript, Altered Metabolic \& Neurochemical Responses to Chronic Unpredictable Stressors in Ghrelin Receptor-Deficient Mice published in the European Jourmal of Neuroscience, as part of his PhD dissertation.

Signature:

Date: Sept $23^{\text {RA }}, 2013$ 
To whom it may concern,

I, Elaine Waddington-Lamont, as a postdoc in Dr. Abizaid Lab, supervised and collaborated with Zachary Patterson in conducting the experiments that are described in the paper entitled Ghrelin-deficient mice have fewer orexin cells and reduced cFOS expression in the mesolimbic dopamine pathway under a restricted feeding paradigm published in Neurssience. Noting that, I hereby grant Zachary Patterson permission to use the contents associated with this manuscript as part of his $\mathrm{PhD}$ dissertation.

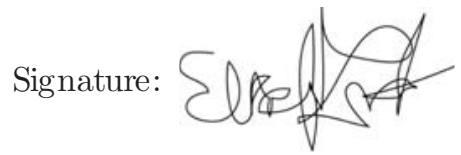

Date: Sept. 23, 2013 
To whom it may concern,

I, Dr. Hymie Anisman, hereby grant Zachary Patterson permission to use all contents associated with the manuscript, Central Ghrelin Signalling Mediates the Metabolic Response of C57 BL6J Male Mice to Chronic Social Defeat Stress published in Endocrinology, as part of his $\mathrm{PhD}$ dissertation.

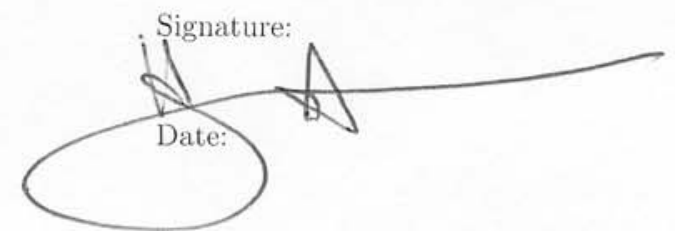


To whom it may concern,

I, Dr. Hymie Anisman, hereby grant Zachary Patterson permission to use all contents associated with the manuscript, Altered Metabolic $\&$ Neurochemical Responses to Chronic Unpredictable Stressors in Ghrelin Receptor-Deficient Mice published in the European Journal of Neuroscience, as part of his PhD dissertation.

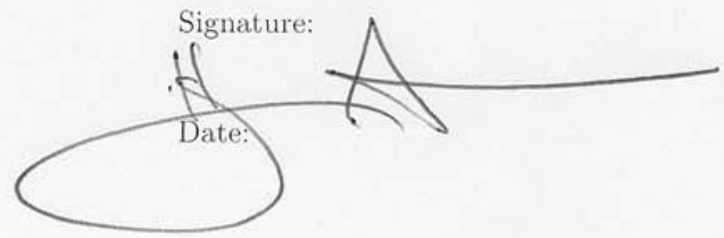


To whom it may concern,

I, Ian David Blum, hereby grant Zachary Patterson permission to use all contents associated with the manuscript, Ghrelin Deficient Mice Have Fewer Orexin Cells 8 Reduced cFo Expression in the Mesolimbic Dopamine Pathway Under a Restricted Feeding Paradigm published in Neuroscience, as part of his $\mathrm{PhD}$ dissertation.

Signature:

Date: Wed Sept 26, 2013 
To when in may concrin.

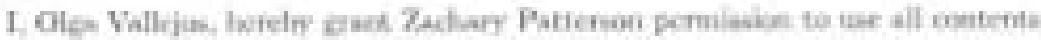

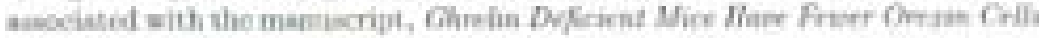

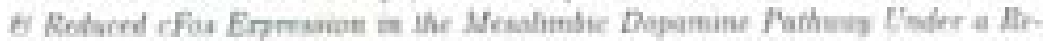

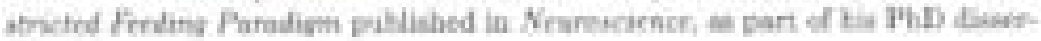
LALivin.

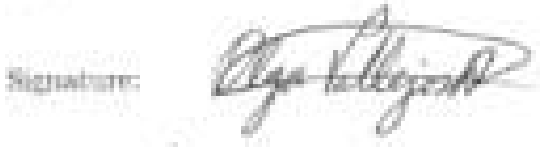

Dater

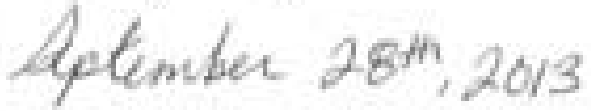


To whom it may concern,

I, Rim Khazall, hereby grant Zachary Patterson permission to use all contents associated with the manuscript, Central Ghrelin Signalling Mediates the Metabolic Response of C57 BL6J Male Mice to Chronic Social Defeat Stress published in Endocrinology, as part of his $\mathrm{PhD}$ dissertation.

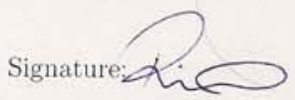

Date: Sept. 23.2013 
'To whom it may oncerm,

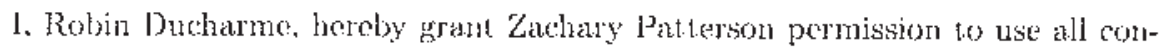

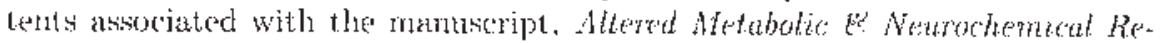

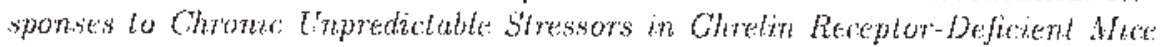

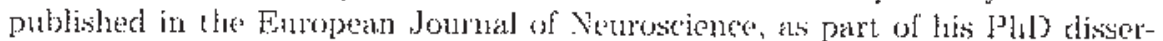
tation.

Sigrature, Polin Aucharme

Date: October 2,2013 
To whom it may concern,

I. Tamara Parno, hereby grant Zachary Patterson permission to use all contents associated with the manuscript, Interruption of Ghrelin Signalling in the PVN Increases High Fat Diet Intake \& Body Weight in Male C57BL6J Mice published in Frontiers in Neuroendocrine Science, as part of his PhD dissertation

Signature: $\mathcal{J a m}_{\text {Pav }}$

Date: Sept 23 
To whom it may concern,

I, Trevor Rodrigues, hereby grant Zachary Patterson permission to use all contents associated with the manuscript, Ghrelin Deficient Mice Have Fewer Orexin Cells 8 Reduced cFos Expression in the Mesolimbic Dopamine Pathway Under a Restricted Feeding Paradigm published in Neuroscience, as part of his PhD dissertation.

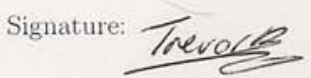

Date: 23 rd September 2013 


\section{Bibliography}

Abercrombie, E. D. and Jacobs, B. L. (1987). Single-unit response of noradrenergic neurons in the locus coeruleus of freely moving cats. I. Acutely presented stressful and nonstressful stimuli. The Journal of neuroscience : the official journal of the Society for Neuroscience, 7(9):2837-43.

Abizaid, A. (2009). Ghrelin and dopamine: new insights on the peripheral regulation of appetite. Journal of neuroendocrinology, 21(9):787-93.

Abizaid, A. and Horvath, T. (2008). Brain Circuits Regulating Energy Homeostasis. Regulatory peptides, 149:3-10.

Abizaid, A. and Liu, Z. (2006). Ghrelin modulates the activity and synaptic input organization of midbrain dopamine neurons while promoting appetite. Journal of Clinical ..., 116(12):3229-3239.

Aguilera, G., Millan, M. A., Hauger, R. L., and Catt, K. J. (1987). Corticotropin-releasing factor receptors: distribution and regulation in brain, pituitary, and peripheral tissues. Annals of the New York Academy of Sciences, 512:48-66.

Aguilera, G., Wynn, P. C., Harwood, J. P., Hauger, R. L., Millan, M. A., Grewe, C., and Catt, K. J. (1986). Receptor-Mediated Actions of CorticotropinReleasing Factor in Pituitary Gland and Nervous System. Neuroendocrinology, 43(1):79-88.

Ahima, R. S., Prabakaran, D., Mantzoros, C., Qu, D., Lowell, B., Maratos-Flier, E., and Flier, J. S. (1996). Role of leptin in the neuroendocrine response to fasting. Nature, 382(6588):250-2.

Akabayashi, A., Koenig, J. I., Watanabe, Y., Alexander, J. T., and Leibowitz, S. F. (1994). Galanin-containing neurons in the paraventricular nucleus: a neurochemical marker for fat ingestion and body weight gain. Proceedings of the National Academy of Sciences of the United States of America, 91(22):10375-9.

Akana, S. F., Strack, A. M., Hanson, E. S., Horsley, C. J., Milligan, E. D., Bhatnagar, S., and Dallman, M. F. (1999). Interactions among chronic cold, 
corticosterone and puberty on energy intake and deposition. Stress (Amsterdam, Netherlands), 3(2):131-46.

Akiyama, M., Yuasa, T., Hayasaka, N., Horikawa, K., Sakurai, T., and Shibata, S. (2004). Reduced food anticipatory activity in genetically orexin (hypocretin) neuron-ablated mice. The European journal of neuroscience, 20(11):3054-62.

Alvarez-Crespo, M., Skibicka, K. P., Farkas, I., Molnár, C. S., Egecioglu, E., Hrabovszky, E., Liposits, Z., and Dickson, S. L. (2012). The amygdala as a neurobiological target for ghrelin in rats: neuroanatomical, electrophysiological and behavioral evidence. PloS one, 7(10):e46321.

Andersson, U., Filipsson, K., Abbott, C. R., Woods, A., Smith, K., Bloom, S. R., Carling, D., and Small, C. J. (2004). AMP-activated Protein Kinase Plays a Role in the Control of Food Intake *. The Journal of biological chemistry, pages $12005-12008$.

Andreis, P. G., Malendowicz, L. K., Trejter, M., Neri, G., Spinazzi, R., Rossi, G. P., and Nussdorfer, G. G. (2003). Ghrelin and growth hormone secretagogue receptor are expressed in the rat adrenal cortex: Evidence that ghrelin stimulates the growth, but not the secretory activity of adrenal cells. FEBS letters, 536(1-3):173-9.

Andrews, Z. B., Liu, Z.-w., Walllingford, N., Erion, D. M., Borok, E., Shanabrough, M., Cline, G., Shulman, G. I., Coppola, A., Friedman, J. M., Tscho, M. H., Diano, S., Gao, X.-b., and Horvath, T. L. (2008). UCP2 mediates ghrelin's action on NPY/AgRP neurons by lowering free radicals. Nature, 454(August).

Angeles-Castellanos, M., Mendoza, J., and Escobar, C. (2007). Restricted feeding schedules phase shift daily rhythms of c-Fos and protein Per1 immunoreactivity in corticolimbic regions in rats. Neuroscience, 144(1):344-55.

Anisman, H. and Matheson, K. (2005). Stress, depression, and anhedonia: caveats concerning animal models. Neuroscience and biobehavioral reviews, 29(4-5):525-46.

Anisman, H., Merali, Z., and Stead, J. D. H. (2008). Experiential and genetic contributions to depressive- and anxiety-like disorders: clinical and experimental studies. Neuroscience and biobehavioral reviews, 32(6):1185-206.

Anisman, H. and Zacharko, R. M. (1990). Multiple neurochemical and behavioral consequences of stressors: implications for depression. Pharmacology $\mathcal{E}$ therapeutics, 46(1):119-36.

Antoni, F. A. (1986). Hypothalamic control of adrenocorticotropin secretion: advances since the discovery of 41-residue corticotropin-releasing factor. Endocrine reviews, 7(4):351-78. 
Aravich, P. F. and Sclafani, A. (1983). Paraventricular hypothalamic lesions and medial hypothalamic knife cuts produce similar hyperphagia syndromes. Behavioral neuroscience, 97(6):970-83.

Ariyasu, H. (2001). Stomach Is a Major Source of Circulating Ghrelin, and Feeding State Determines Plasma Ghrelin-Like Immunoreactivity Levels in Humans. Journal of Clinical Endocrinology 83 Metabolism, 86(10):4753-4758.

Arnsten, A. F. (2000). Stress impairs prefrontal cortical function in rats and monkeys: role of dopamine D1 and norepinephrine alpha-1 receptor mechanisms. Progress in brain research, 126:183-92.

Arnsten, A. F. T. (2009). Stress signalling pathways that impair prefrontal cortex structure and function. Nature reviews. Neuroscience, 10(6):410-22.

Aronsson, M. (1988). Localization of Glucocorticoid Receptor mRNA in the Male Rat Brain by in situ Hybridization. Proceedings of the National Academy of Sciences, 85(23):9331-9335.

Arriza, J. L., Simerly, R. B., Swanson, L. W., and Evans, R. M. (1988). The neuronal mineralocorticoid receptor as a mediator of glucocorticoid response. Neuron, 1(9):887-900.

Asakawa, A., Inui, A., Kaga, T., Yuzuriha, H., Nagata, T., Fujimiya, M., Katsuura, G., Makino, S., Fujino, M. A., and Kasuga, M. (2001a). A role of ghrelin in neuroendocrine and behavioral responses to stress in mice. Neuroendocrinology, 74(3):143-7.

Asakawa, A., Inui, A., Kaga, T., Yuzuriha, H., Nagata, T., Ueno, N., Makino, S., Fujimiya, M., Niijima, A., Fujino, M. A., and Kasuga, M. (2001b). Ghrelin is an appetite-stimulatory signal from stomach with structural resemblance to motilin. Gastroenterology, 120(2):337-45.

Aston-Jones, G., Chiang, C., and Alexinsky, T. (1991). Discharge of noradrenergic locus coeruleus neurons in behaving rats and monkeys suggests a role in vigilance. Progress in brain research, 88:501-20.

Atasoy, D., Betley, J. N., Su, H. H., and Sternson, S. M. (2012). Deconstruction of a neural circuit for hunger. Nature, 488(7410):172-7.

Baler, R. D. and Volkow, N. D. (2006). Drug addiction: the neurobiology of disrupted self-control. Trends in molecular medicine, 12(12):559-66.

Bamshad, M., Aoki, V. T., Adkison, M. G., Warren, W. S., and Bartness, T. J. (1998). Central nervous system origins of the sympathetic nervous system outflow to white adipose tissue. The American journal of physiology, 275(1 Pt 2):R291-9.

Barnett, S. A. (1958). Physiological effects of social stress in wild rats. I. The adrenal cortex. Journal of psychosomatic research, 3(1):1-11. 
Barnett, S. A., EATON, J. C., and McCallum, H. M. (1960). Physiological effects of "social stress" in wild rats-II. Liver glycogen and blood glucose. Journal of psychosomatic research, 4:251-60.

Bartolomucci, A. (2010). Increased vulnerability to psychosocial stress in heterozygous serotonin transporter knockout mice. Dis Model ..., 000:1-12.

Bartolomucci, A., Cabassi, A., and Govoni, P. (2009). Metabolic consequences and vulnerability to diet-induced obesity in male mice under chronic social stress. PLoS One, 4(1).

Bartolomucci, A. and Palanza, P. (2005). Social factors and individual vulnerability to chronic stress exposure. Neuroscience $\mathcal{E}$. . ., 29:67-81.

Bartolomucci, a., Palanza, P., Gaspani, L., Limiroli, E., Panerai, a. E., Ceresini, G., Poli, M. D., and Parmigiani, S. (2001). Social status in mice: behavioral, endocrine and immune changes are context dependent. Physiology $\& 3$ behavior, 73(3):401-10.

Bartolomucci, A. and Pederzani, T. (2004). Behavioral and physiological characterization of male mice under chronic psychosocial stress. Psychoneuroendocrinology, pages 899-910.

Beck, B. and Max, J.-P. (2008). Neuropeptide Y in the arcuato-paraventricular pathway and diet selection in the vasopressin-deficient Brattleboro rat. Brain research bulletin, 76(4):454-7.

Bednarek, M. A., Feighner, S. D., Pong, S. S., McKee, K. K., Hreniuk, D. L., Silva, M. V., Warren, V. A., Howard, A. D., Van Der Ploeg, L. H., and Heck, J. V. (2000). Structure-function studies on the new growth hormonereleasing peptide, ghrelin: minimal sequence of ghrelin necessary for activation of growth hormone secretagogue receptor 1a. Journal of medicinal chemistry, 43(23):4370-6.

Berthoud, H.-R. (2002). Multiple neural systems controlling food intake and body weight. Neuroscience and biobehavioral reviews, 26(4):393-428.

Berthoud, H.-R., Sutton, G. M., Townsend, R. L., Patterson, L. M., and Zheng, H. (2006). Brainstem mechanisms integrating gut-derived satiety signals and descending forebrain information in the control of meal size. Physiology 83 behavior, 89(4):517-24.

Berton, O., Aguerre, S., Sarrieau, A., Mormede, P., and Chaouloff, F. (1998). Differential effects of social stress on central serotonergic activity and emotional reactivity in Lewis and spontaneously hypertensive rats. Neuroscience, 82(1):147-59.

Bhatnagar, S. and Vining, C. (2003). Facilitation of hypothalamic-pituitaryadrenal responses to novel stress following repeated social stress using the resident/intruder paradigm. Hormones and behavior, 43(1):158-65. 
Birnbaum, S., Gobeske, K. T., Auerbach, J., Taylor, J. R., and Arnsten, A. F. (1999). A role for norepinephrine in stress-induced cognitive deficits: alpha1-adrenoceptor mediation in the prefrontal cortex. Biological psychiatry, 46(9):1266-74.

Björntorp, P. (2001). Do stress reactions cause abdominal obesity and comorbidities? Obesity reviews : an official journal of the International Association for the Study of Obesity, 2(2):73-86.

Björntorp, P. and Rosmond, R. (1999). Hypothalamic origin of the metabolic syndrome X. Annals of the New York Academy of Sciences, 892:297-307.

Björntorp, P. and Rosmond, R. (2000). The metabolic syndrome-a neuroendocrine disorder? The British journal of nutrition, 83 Suppl 1:S49-57.

Black, P. (2006). The Inflammatory consequences of psychological stress: relationship to insulin resistance, obesity, atherosclerosis and diabetes mellitus, type II. Medical hypotheses, 67(4):879-91.

Blackburn, J. R., Pfaus, J. G., and Phillips, A. G. (1992). Dopamine functions in appetitive and defensive behaviours. Progress in neurobiology, 39(3):247-79.

Blanchard, D. and Spencer, R. (1995). Visible burrow system as a model of chronic social stress: behavioral and neuroendocrine correlates. Psychoneuroendocrinology, 20(2):117-134.

Blanchard, D. C., Sakai, R. R., McEwen, B., Weiss, S. M., and Blanchard, R. J. (1993). Subordination stress: behavioral, brain, and neuroendocrine correlates. Behavioural brain research, 58(1-2):113-21.

Blanchard, R. J., Blanchard, D. C., Takahashi, T., and Kelley, M. J. (1977). Attack and defensive behaviour in the albino rat. Animal behaviour, 25(3):62234 .

Blum, I. D., Patterson, Z., Khazall, R., Lamont, E. W., Sleeman, M. W., Horvath, T. L., and Abizaid, a. (2009). Reduced anticipatory locomotor responses to scheduled meals in ghrelin receptor deficient mice. Neuroscience, 164(2):351-9.

Bondensgaard, K., Ankersen, M., Thø gersen, H., Hansen, B. S., Wulff, B. S., and Bywater, R. P. (2004). Recognition of privileged structures by G-protein coupled receptors. Journal of medicinal chemistry, 47(4):888-99.

Borgland, S. L., Chang, S.-J., Bowers, M. S., Thompson, J. L., Vittoz, N., Floresco, S. B., Chou, J., Chen, B. T., and Bonci, A. (2009). Orexin A/hypocretin-1 selectively promotes motivation for positive reinforcers. The Journal of neuroscience : the official journal of the Society for Neuroscience, 29(36):11215-25. 
Borgland, S. L., Taha, S. A., Sarti, F., Fields, H. L., and Bonci, A. (2006). Orexin A in the VTA is critical for the induction of synaptic plasticity and behavioral sensitization to cocaine. Neuron, 49(4):589-601.

Boudaba, C., Szabó, K., and Tasker, J. G. (1996). Physiological mapping of local inhibitory inputs to the hypothalamic paraventricular nucleus. The Journal of neuroscience : the official journal of the Society for Neuroscience, 16(22):7151-60.

Bray, G. A. (2000). Afferent signals regulating food intake. The Proceedings of the Nutrition Society, 59(3):373-84.

Bremner, J. D., Krystal, J. H., Southwick, S. M., and Charney, D. S. (1996). Noradrenergic mechanisms in stress and anxiety: I. Preclinical studies. Synapse (New York, N.Y.), 23(1):28-38.

Brennan, I. M., Otto, B., Feltrin, K. L., Meyer, J. H., Horowitz, M., and FeinleBisset, C. (2007). Intravenous CCK-8, but not GLP-1, suppresses ghrelin and stimulates PYY release in healthy men. Peptides, 28(3):607-11.

Brindley, D. N. and Rolland, Y. (1989). Possible connections between stress, diabetes, obesity, hypertension and altered lipoprotein metabolism that may result in atherosclerosis. Clinical science (London, England : 1979), 77(5):45361.

Broberger, C., Landry, M., Wong, H., Walsh, J. N., and Hökfelt, T. (1997). Subtypes Y1 and Y2 of the neuropeptide Y receptor are respectively expressed in pro-opiomelanocortin- and neuropeptide-Y-containing neurons of the rat hypothalamic arcuate nucleus. Neuroendocrinology, 66(6):393-408.

Broglio, F., Gottero, C., Benso, A., Prodam, F., Destefanis, S., Gauna, C., Maccario, M., Deghenghi, R., van der Lely, A. J., and Ghigo, E. (2003). Effects of ghrelin on the insulin and glycemic responses to glucose, arginine, or free fatty acids load in humans. The Journal of clinical endocrinology and metabolism, 88(9):4268-72.

Brown, P. and Molliver, M. E. (2000). Dual serotonin (5-HT) projections to the nucleus accumbens core and shell: relation of the 5-HT transporter to amphetamine-induced neurotoxicity. The Journal of neuroscience : the official journal of the Society for Neuroscience, 20(5):1952-63.

Brunetti, L., Recinella, L., and Orlando, G. (2002). Effects of ghrelin and amylin on dopamine, norepinephrine and serotonin release in the hypothalamus. European journal of ..., 454:189-192.

Brunner, E. J., Chandola, T., and Marmot, M. G. (2007). Prospective effect of job strain on general and central obesity in the Whitehall II Study. American journal of epidemiology, 165(7):828-37. 
Buckingham, J. C. (2006). Glucocorticoids: exemplars of multi-tasking. British journal of pharmacology, 147 Suppl:S258-68.

Burdette, A. M. and Hill, T. D. (2008). An examination of processes linking perceived neighborhood disorder and obesity. Social science 83 medicine (1982), 67(1):38-46.

Butcher, R. W., Robison, G. A., Hardman, J. G., and Sutherland, E. W. (1968). The role of cyclic AMP in hormone actions. Advances in enzyme regulation, 6:357-89.

Cabral, A., Suescun, O., Zigman, J. M., and Perello, M. (2012). Ghrelin indirectly activates hypophysiotropic CRF neurons in rodents. PloS one, $7(2): \mathrm{e} 31462$.

Cai, Y., Hay, M., and Bishop, V. S. (1996). Synaptic connections and interactions between area postrema and nucleus tractus solitarius. Brain research, 724(1):121-4.

Calogero, A. E., Gallucci, W. T., Chrousos, G. P., and Gold, P. W. (1988). Catecholamine effects upon rat hypothalamic corticotropin-releasing hormone secretion in vitro. The Journal of clinical investigation, 82(3):839-46.

Camiña, J. P. (2006). Cell biology of the ghrelin receptor. Journal of neuroendocrinology, 18(1):65-76.

Camiña, J. P., Carreira, M. C., El Messari, S., Llorens-Cortes, C., Smith, R. G., and Casanueva, F. F. (2004). Desensitization and endocytosis mechanisms of ghrelin-activated growth hormone secretagogue receptor 1a. Endocrinology, 145(2):930-40.

Campbell, J. E., Peckett, A. J., D'souza, A. M., Hawke, T. J., and Riddell, M. C. (2011). Adipogenic and lipolytic effects of chronic glucocorticoid exposure. American journal of physiology. Cell physiology, 300(1):C198-209.

Cannon, W. (1929). Organization for physiological homeostasis. Physiological Reviews, IX(3):399-431.

Carlini, V. P., Monzón, M. E., Varas, M. M., Cragnolini, A. B., Schiöth, H. B., Scimonelli, T. N., and de Barioglio, S. R. (2002). Ghrelin increases anxiety-like behavior and memory retention in rats. Biochemical and biophysical research communications, 299(5):739-43.

Carlini, V. P., Varas, M. M., Cragnolini, A. B., Schiöth, H. B., Scimonelli, T. N., and de Barioglio, S. R. (2004). Differential role of the hippocampus, amygdala, and dorsal raphe nucleus in regulating feeding, memory, and anxiety-like behavioral responses to ghrelin. Biochemical and biophysical research communications, 313(3):635-41. 
Cartwright, M., Wardle, J., Steggles, N., Simon, A. E., Croker, H., and Jarvis, M. J. (2003). Stress and dietary practices in adolescents. Health psychology: official journal of the Division of Health Psychology, American Psychological Association, 22(4):362-9.

Caruso, C. C. (2006). Possible broad impacts of long work hours. Industrial health, 44(4):531-6.

Carvajal, P., Carlini, V. P., Schiöth, H. B., de Barioglio, S. R., and Salvatierra, N. A. (2009). Central ghrelin increases anxiety in the Open Field test and impairs retention memory in a passive avoidance task in neonatal chicks. Neurobiology of learning and memory, 91(4):402-7.

Castañeyra Perdomo, A., Pérez-Delgado, M. M., Montagnese, C., and Coen, C. W. (1992). Brainstem projections to the medial preoptic region containing the luteinizing hormone-releasing hormone perikarya in the rat. An immunohistochemical and retrograde transport study. Neuroscience letters, 139(1):135-9.

Castonguay, T. W. (1991). Glucocorticoids as modulators in the control of feeding. Brain research bulletin, 27(3-4):423-8.

Cavagnini, F., Croci, M., Putignano, P., Petroni, M. L., and Invitti, C. (2000). Glucocorticoids and neuroendocrine function. International journal of obesity and related metabolic disorders : journal of the International Association for the Study of Obesity, 24 Suppl 2:S77-9.

Cecchi, M., Khoshbouei, H., Javors, M., and Morilak, D. A. (2002). Modulatory effects of norepinephrine in the lateral bed nucleus of the stria terminalis on behavioral and neuroendocrine responses to acute stress. Neuroscience, 112(1):13-21.

Chagra, S. L., Zavala, J. K., Hall, M. V., and Gosselink, K. L. (2011). Acute and repeated restraint differentially activate orexigenic pathways in the rat hypothalamus. Regulatory peptides, 167(1):70-8.

Chalmers, D. T., Lovenberg, T. W., and De Souza, E. B. (1995). Localization of novel corticotropin-releasing factor receptor (CRF2) mRNA expression to specific subcortical nuclei in rat brain: comparison with CRF1 receptor mRNA expression. The Journal of neuroscience : the official journal of the Society for Neuroscience, 15(10):6340-50.

Chao, H., Digruccio, M., Chen, P., and Li, C. (2012). Type 2 corticotropinreleasing factor receptor in the ventromedial nucleus of hypothalamus is critical in regulating feeding and lipid metabolism in white adipose tissue. Endocrinology, 153(1):166-76.

Chen, A., Zorrilla, E., Smith, S., Rousso, D., Levy, C., Vaughan, J., Donaldson, C., Roberts, A., Lee, K.-F., and Vale, W. (2006). Urocortin 2-deficient 
mice exhibit gender-specific alterations in circadian hypothalamus-pituitaryadrenal axis and depressive-like behavior. The Journal of neuroscience : the official journal of the Society for Neuroscience, 26(20):5500-10.

Chen, P., Vaughan, J., Donaldson, C., Vale, W., and Li, C. (2010). Injection of Urocortin 3 into the ventromedial hypothalamus modulates feeding, blood glucose levels, and hypothalamic POMC gene expression but not the HPA axis. American journal of physiology. Endocrinology and metabolism, 298(2):E337-45.

Choi, K., Roh, S., and Hong, Y. (2003). The role of ghrelin and growth hormone secretagogues receptor on rat adipogenesis. Endocrinology, 144:754-759.

Chrousos, G. P. and Gold, P. W. (1998). A healthy body in a healthy mindand vice versa-the damaging power of "uncontrollable" stress. The Journal of clinical endocrinology and metabolism, 83(6):1842-5.

Chuang, J. and Perello, M. (2011). Ghrelin mediates stress-induced food-reward behavior in mice. The Journal of .., 121(7).

Chuang, J.-c., Krishnan, V., Yu, H. G., Mason, B., Cui, H., Wilkinson, M. B., Zigman, J. M., Elmquist, J. K., Nestler, E. J., and Lutter, M. (2010). Regulates Behavioral and Metabolic Changes Induced by Chronic Stress. BPS.

Ciofi, P. (2011). The arcuate nucleus as a circumventricular organ in the mouse. Neuroscience letters, 487(2):187-90.

Clark, J. T., Kalra, P. S., Crowley, W. R., and Kalra, S. P. (1984). Neuropeptide $\mathrm{Y}$ and human pancreatic polypeptide stimulate feeding behavior in rats. Endocrinology, 115(1):427-9.

Coccurello, R., D'Amato, F., and Moles, A. (2008). Chronic social stress, hedonism and vulnerability to obesity: Lessons from Rodents. Neuroscience 8 Biobehavioral ..., 33:537-550.

Cole, M. A., Kim, P. J., Kalman, B. A., and Spencer, R. L. (2000). Dexamethasone suppression of corticosteroid secretion: evaluation of the site of action by receptor measures and functional studies. Psychoneuroendocrinology, 25(2):151-67.

Cone, R. D., Cowley, M. A., Butler, A. A., Fan, W., Marks, D. L., and Low, M. J. (2001). The arcuate nucleus as a conduit for diverse signals relevant to energy homeostasis. International journal of obesity and related metabolic disorders : journal of the International Association for the Study of Obesity, 25 Suppl 5:S63-7.

Coste, S. C., Kesterson, R. A., Heldwein, K. A., Stevens, S. L., Heard, A. D., Hollis, J. H., Murray, S. E., Hill, J. K., Pantely, G. A., Hohimer, A. R., Hatton, D. C., Phillips, T. J., Finn, D. A., Low, M. J., Rittenberg, M. B., Stenzel, P., and Stenzel-Poore, M. P. (2000). Abnormal adaptations to stress 
and impaired cardiovascular function in mice lacking corticotropin-releasing hormone receptor-2. Nature genetics, 24(4):403-9.

Cowley, M. A., Smart, J. L., Rubinstein, M., Cerdán, M. G., Diano, S., Horvath, T. L., Cone, R. D., and Low, M. J. (2001). Leptin activates anorexigenic POMC neurons through a neural network in the arcuate nucleus. Nature, 411(6836):480-4.

Cowley, M. A., Smith, R. G., Diano, S., Tschöp, M., Pronchuk, N., Grove, K. L., Strasburger, C. J., Bidlingmaier, M., Esterman, M., Heiman, M. L., Garcia-Segura, L. M., Nillni, E. A., Mendez, P., Low, M. J., Sotonyi, P., Friedman, J. M., Liu, H., Pinto, S., Colmers, W. F., Cone, R. D., and Horvath, T. L. (2003). The distribution and mechanism of action of ghrelin in the CNS demonstrates a novel hypothalamic circuit regulating energy homeostasis. Neuron, 37(4):649-61.

Crowley, V. E. F., Yeo, G. S. H., and O'Rahilly, S. (2002). Obesity therapy: altering the energy intake-and-expenditure balance sheet. Nature reviews. Drug discovery, 1(4):276-86.

Cummings, D. E., Purnell, J. Q., Frayo, R. S., Schmidova, K., Wisse, B. E., and Weigle, D. S. (2001). A preprandial rise in plasma ghrelin levels suggests a role in meal initiation in humans. Diabetes, 50(8):1714-9.

Cummings, D. E. and Shannon, M. H. (2003). Roles for ghrelin in the regulation of appetite and body weight. Archives of surgery (Chicago, Ill. : 1960), 138(4):389-96.

Cunningham, E. T., Bohn, M. C., and Sawchenko, P. E. (1990). Organization of adrenergic inputs to the paraventricular and supraoptic nuclei of the hypothalamus in the rat. The Journal of comparative neurology, 292(4):651-67.

Cunningham, E. T. and Sawchenko, P. E. (1988). Anatomical specificity of noradrenergic inputs to the paraventricular and supraoptic nuclei of the rat hypothalamus. The Journal of comparative neurology, 274(1):60-76.

Currie, P., Mirza, A., and Fuld, R. (2005). Ghrelin is an orexigenic and metabolic signaling peptide in the arcuate and paraventricular nuclei. American Journal of ..., pages 353-358.

Currie, P. J., Khelemsky, R., Rigsbee, E. M., Dono, L. M., Coiro, C. D., Chapman, C. D., and Hinchcliff, K. (2012). Ghrelin is an orexigenic peptide and elicits anxiety-like behaviors following administration into discrete regions of the hypothalamus. Behavioural brain research, 226(1):96-105.

Dal-Zotto, S., Martí, O., Delgado, R., and Armario, A. (2004). Potentiation of glucocorticoid release does not modify the long-term effects of a single exposure to immobilization stress. Psychopharmacology, 177(1-2):230-7. 
Dalla, C., Antoniou, K., Drossopoulou, G., Xagoraris, M., Kokras, N., Sfikakis, a., and Papadopoulou-Daifoti, Z. (2005). Chronic mild stress impact: are females more vulnerable? Neuroscience, 135(3):703-14.

Dalla, C., Pitychoutis, P. M., Kokras, N., and Papadopoulou-Daifoti, Z. (2010). Sex differences in animal models of depression and antidepressant response. Basic $\& 3$ clinical pharmacology $\& 3$ toxicology, 106(3):226-33.

Dallman, M. and Pecoraro, N. (2003). Chronic stress and obesity: a new view of comfort food. Proceedings of the ....

Dallman, M. and Warne, J. (2007). Glucocorticoids and insulin both modulate caloric intake through actions on the brain. The Journal of . ., 2:431-436.

Dallman, M. F., Pecoraro, N. C., and Fleur, S. E. (2005). Chronic stress and comfort foods: Self-medication and abdominal obesity . BRAIN, BEHAVIOUR, AND IMMUNITY, 19:275-280.

Dallman, M. F., Pecoraro, N. C., La Fleur, S. E., Warne, J. P., Ginsberg, A. B., Akana, S. F., Laugero, K. C., Houshyar, H., Strack, A. M., Bhatnagar, S., and Bell, M. E. (2006). Glucocorticoids, chronic stress, and obesity. Progress in brain research, 153:75-105.

Dallman, M. F., Strack, A. M., Akana, S. F., Bradbury, M. J., Hanson, E. S., Scribner, K. A., and Smith, M. (1993). Feast and famine: critical role of glucocorticoids with insulin in daily energy flow. Frontiers in neuroendocrinology, 14(4):303-47.

Daniels, W. M., Jaffer, A., Russell, V. A., and Taljaard, J. J. (1993). Alpha 2 - and beta-adrenergic stimulation of corticosterone secretion in rats. Neurochemical research, 18(2):159-64.

Date, Y., Murakami, N., Toshinai, K., Matsukura, S., Niijima, A., Matsuo, H., Kangawa, K., and Nakazato, M. (2002). The role of the gastric afferent vagal nerve in ghrelin-induced feeding and growth hormone secretion in rats. Gastroenterology, 123(4):1120-1128.

Date, Y., Shimbara, T., Koda, S., and Toshinai, K. (2006). Peripheral ghrelin transmits orexigenic signals through the noradrenergic pathway from the hindbrain to the hypothalamus. Cell metabolism, (October):323-331.

Davies, J. S., Kotokorpi, P., Eccles, S. R., Barnes, S. K., Tokarczuk, P. F., Allen, S. K., Whitworth, H. S., Guschina, I. A., Evans, B. A. J., Mode, A., Zigman, J. M., and Wells, T. (2009). Ghrelin induces abdominal obesity via GHS-R-dependent lipid retention. Molecular endocrinology (Baltimore, Md.), 23(6):914-24.

Davis, J. F., Perello, M., Choi, D. L., Magrisso, I. J., Kirchner, H., Pfluger, P. T., Tschoep, M., Zigman, J. M., and Benoit, S. C. (2012). GOAT induced ghrelin acylation regulates hedonic feeding. Hormones and behavior, 62(5):598-604. 
Day, H. E., Campeau, S., Watson, S. J., and Akil, H. (1997). Distribution of alpha 1a-, alpha 1b- and alpha 1d-adrenergic receptor mRNA in the rat brain and spinal cord. Journal of chemical neuroanatomy, 13(2):115-39.

Dayas, C. V., Buller, K. M., Crane, J. W., Xu, Y., and Day, T. a. (2001). Stressor categorization: acute physical and psychological stressors elicit distinctive recruitment patterns in the amygdala and in medullary noradrenergic cell groups. The European journal of neuroscience, 14(7):1143-52.

de la Cour, C. D., Norlén, P., and Hå kanson, R. (2007). Secretion of ghrelin from rat stomach ghrelin cells in response to local microinfusion of candidate messenger compounds: a microdialysis study. Regulatory peptides, 143(1$3): 118-26$.

De Souza, E. B. and Kuhar, M. J. (1986). Corticotropin-releasing factor receptors: autoradiographic identification. Research publications - Association for Research in Nervous and Mental Disease, 64:179-98.

Delhanty, P., Sun, Y., and Visser, J. (2010a). Unacylated ghrelin rapidly modulates lipogenic and insulin signaling pathway gene expression in metabolically active tissues of GHSR deleted mice. PLoS One, 5(7).

Delhanty, P. J. D., van Kerkwijk, A., Huisman, M., van de Zande, B., VerhoefPost, M., Gauna, C., Hofland, L., Themmen, A. P. N., and van der Lely, a. J. (2010b). Unsaturated fatty acids prevent desensitization of the human growth hormone secretagogue receptor by blocking its internalization. American journal of physiology. Endocrinology and metabolism, 299(3):E497-505.

Depke, M., Fusch, G., and Domanska, G. (2008). Hypermetabolic syndrome as a consequence of repeated psychological stress in mice. Endocrinology, 149(6):2714-2723.

Depoortere, I., Thijs, T., and Peeters, T. (2006). The contractile effect of the ghrelin receptor antagonist, D-Lys3-GHRP-6, in rat fundic strips is mediated through 5-HT receptors. European journal of pharmacology, 537(1-3):160-5.

Devoino, L., Alperina, E., and Pavina, T. (2003). Immunological consequences of the reversal of social status in C57BL/6J mice. Brain, behavior, and immunity, 17(1):28-34.

Dhillo, W. S. and Bloom, S. R. (2001). Hypothalamic peptides as drug targets for obesity. Current opinion in pharmacology, 1(6):651-5.

Di, S., Malcher-Lopes, R., Halmos, K. C., and Tasker, J. G. (2003). Nongenomic glucocorticoid inhibition via endocannabinoid release in the hypothalamus: a fast feedback mechanism. The Journal of neuroscience : the official journal of the Society for Neuroscience, 23(12):4850-7. 
Di, S., Malcher-Lopes, R., Marcheselli, V. L., Bazan, N. G., and Tasker, J. G. (2005). Rapid glucocorticoid-mediated endocannabinoid release and opposing regulation of glutamate and gamma-aminobutyric acid inputs to hypothalamic magnocellular neurons. Endocrinology, 146(10):4292-301.

Diano, S., Farr, S. A., Benoit, S. C., McNay, E. C., da Silva, I., Horvath, B., Gaskin, F. S., Nonaka, N., Jaeger, L. B., Banks, W. A., Morley, J. E., Pinto, S., Sherwin, R. S., Xu, L., Yamada, K. A., Sleeman, M. W., Tschöp, M. H., and Horvath, T. L. (2006). Ghrelin controls hippocampal spine synapse density and memory performance. Nature neuroscience, 9(3):381-8.

Díaz-Regueira, S. and Anadón, R. (1992). Central projections of the vagus nerve in Chelon labrosus Risso (Teleostei, O. Perciformes). Brain, behavior and evolution, 40(6):297-310.

Dinneen, S., Alzaid, A., Miles, J., and Rizza, R. (1993). Metabolic effects of the nocturnal rise in cortisol on carbohydrate metabolism in normal humans. The Journal of clinical investigation, 92(5):2283-90.

Diorio, D., Viau, V., and Meaney, M. (1993). The Role of the Medial Prefrontal Cortex (Cingulate Gyrus) in the Regulation of HypothalamicPituitary-Adrenal Responses to Stress. The Journal of neuroscience, 13(September):3839-3847.

Dornonville de la Cour, C., Björkqvist, M., Sandvik, A. K., Bakke, I., Zhao, C. M., Chen, D., and Hå kanson, R. (2001). A-like cells in the rat stomach contain ghrelin and do not operate under gastrin control. Regulatory peptides, 99(2-3):141-50.

Drazen, D. L., Vahl, T. P., D'Alessio, D. A., Seeley, R. J., and Woods, S. C. (2006). Effects of a fixed meal pattern on ghrelin secretion: evidence for a learned response independent of nutrient status. Endocrinology, 147(1):23-30.

Duncko, R., Kiss, A., Skultétyová, I., Rusnák, M., and Jezová, D. (2001). Corticotropin-releasing hormone mRNA levels in response to chronic mild stress rise in male but not in female rats while tyrosine hydroxylase mRNA levels decrease in both sexes. Psychoneuroendocrinology, 26(1):77-89.

Dunn, A. J., Swiergiel, A. H., and Palamarchouk, V. (2004). Brain circuits involved in corticotropin-releasing factor-norepinephrine interactions during stress. Annals of the New York Academy of Sciences, 1018:25-34.

Dunn, J. D. and Orr, S. E. (1984). Differential plasma corticosterone responses to hippocampal stimulation. Experimental brain research. Experimentelle Hirnforschung. Expérimentation cérébrale, 54(1):1-6.

Elias, C. F., Lee, C., Kelly, J., Aschkenasi, C., Ahima, R. S., Couceyro, P. R., Kuhar, M. J., Saper, C. B., and Elmquist, J. K. (1998). Leptin activates hypothalamic CART neurons projecting to the spinal cord. Neuron, 21(6):137585 . 
Ellenberger, H. H. and Feldman, J. L. (1990). Brainstem connections of the rostral ventral respiratory group of the rat. Brain research, 513(1):35-42.

Elmquist, J. K. (2000). Anatomic basis of leptin action in the hypothalamus. Frontiers of hormone research, 26:21-41.

Esposito, M., Pellinen, J., Kapás, L., and Szentirmai, E. (2012). Impaired wake-promoting mechanisms in ghrelin receptor-deficient mice. The European journal of neuroscience, 35(2):233-43.

Fadel, J. and Deutch, A. Y. (2002). Anatomical substrates of orexin-dopamine interactions: lateral hypothalamic projections to the ventral tegmental area. Neuroscience, 111(2):379-87.

Farias-Silva, E., Sampaio-Barros, M. M., Amaral, M. E. C., Carneiro, E. M., Boschero, A. C., Grassi-Kassisse, D. M., and Spadari-Bratfisch, R. C. (2002). Subsensitivity to insulin in adipocytes from rats submitted to foot-shock stress. Canadian journal of physiology and pharmacology, 80(8):783-9.

Faulconbridge, L. F., Cummings, D. E., Kaplan, J. M., and Grill, H. J. (2003). Hyperphagic effects of brainstem ghrelin administration. Diabetes, $52(9): 2260-5$.

Feighner, S. D., Howard, A. D., Prendergast, K., Palyha, O. C., Hreniuk, D. L., Nargund, R., Underwood, D., Tata, J. R., Dean, D. C., Tan, C. P., McKee, K. K., Woods, J. W., Patchett, A. A., Smith, R. G., and Van der Ploeg, L. H. (1998). Structural requirements for the activation of the human growth hormone secretagogue receptor by peptide and nonpeptide secretagogues. Molecular endocrinology (Baltimore, Md.), 12(1):137-45.

Fendler, K., Karmos, G., and Telegdy, G. (1961). The effect of hippocampal lesion on pituitary-adrenal function. Acta physiologica Academiae Scientiarum Hungaricae, 20:293-7.

Ferguson, A. V. (1991). The area postrema: a cardiovascular control centre at the blood-brain interface? Canadian journal of physiology and pharmacology, 69(7):1026-34.

Fields, H. L., Hjelmstad, G. O., Margolis, E. B., and Nicola, S. M. (2007). Ventral tegmental area neurons in learned appetitive behavior and positive reinforcement. Annual review of neuroscience, 30:289-316.

Figueiredo, H. F., Bodie, B. L., Tauchi, M., Dolgas, C. M., and Herman, J. P. (2003a). Stress integration after acute and chronic predator stress: differential activation of central stress circuitry and sensitization of the hypothalamopituitary-adrenocortical axis. Endocrinology, 144(12):5249-58.

Figueiredo, H. F., Bruestle, A., Bodie, B., Dolgas, C. M., and Herman, J. P. (2003b). The medial prefrontal cortex differentially regulates stress-induced c-fos expression in the forebrain depending on type of stressor. European Journal of Neuroscience, 18(8):2357-2364. 
Flegal, K. M., Carroll, M. D., Kit, B. K., and Ogden, C. L. (2012). Prevalence of obesity and trends in the distribution of body mass index among US adults, 1999-2010. JAMA : the journal of the American Medical Association, 307(5):491-7.

Flegal, K. M., Carroll, M. D., Ogden, C. L., and Curtin, L. R. (2010). Prevalence and trends in obesity among US adults, 1999-2008. JAMA : the journal of the American Medical Association, 303(3):235-41.

Foster, M. and Solomon, M. (2006). Social defeat increases food intake, body mass, and adiposity in Syrian hamsters. American Journal of ..., pages $1284-1293$.

Fraley, G. S., Dinh, T. T., and Ritter, S. (2002). Immunotoxic catecholamine lesions attenuate 2DG-induced increase of AGRP mRNA. Peptides, 23(6):10939 .

Fraley, G. S. and Ritter, S. (2003). Immunolesion of norepinephrine and epinephrine afferents to medial hypothalamus alters basal and 2-deoxy-Dglucose-induced neuropeptide $\mathrm{Y}$ and agouti gene-related protein messenger ribonucleic acid expression in the arcuate nucleus. Endocrinology, 144(1):7583.

Frasure-Smith, N. and Lespérance, F. (2005). Reflections on depression as a cardiac risk factor. Psychosomatic medicine, 67 Suppl 1:S19-25.

Fried, S. K., Bunkin, D. A., and Greenberg, A. S. (1998). Omental and subcutaneous adipose tissues of obese subjects release interleukin-6: depot difference and regulation by glucocorticoid. The Journal of clinical endocrinology and metabolism, 83(3):847-50.

Friedman, J. M. (2002). The function of leptin in nutrition, weight, and physiology. Nutrition reviews, 60(10 Pt 2):S1-14; discussion S68-84, 85-7.

Furay, A. R., Bruestle, A. E., and Herman, J. P. (2008). The role of the forebrain glucocorticoid receptor in acute and chronic stress. Endocrinology, 149(11):5482-90.

Ganong, W. F., Chalett, J., Jones, H., Kaplan, S. L., Karteszi, M., Stith, R. D., and Van de Kar, L. D. (1982). Further characterization of putative alphaadrenergic receptors in brain that affect blood pressure and the secretion of ACTH, GH and renin in dogs. Endocrinologia experimentalis, 16(3-4):191205.

Gao, Q. and Horvath, T. L. (2007). Neurobiology of feeding and energy expenditure. Annual review of neuroscience, 30:367-98.

Geliebter, A., Gibson, C. D., Hernandez, D. B., Atalayer, D., Kwon, A., Lee, M. I., Mehta, N., Phair, D., and Gluck, M. E. (2012). Plasma cortisol levels in response to a cold pressor test did not predict appetite or ad libitum test meal intake in obese women. Appetite, 59(3):956-9. 
George, G. C., Milani, T. J., Hanss-Nuss, H., and Freeland-Graves, J. H. (2005). Compliance with dietary guidelines and relationship to psychosocial factors in low-income women in late postpartum. Journal of the American Dietetic Association, 105(6):916-26.

Gerald, C., Walker, M. W., Criscione, L., Gustafson, E. L., Batzl-Hartmann, C., Smith, K. E., Vaysse, P., Durkin, M. M., Laz, T. M., Linemeyer, D. L., Schaffhauser, A. O., Whitebread, S., Hofbauer, K. G., Taber, R. I., Branchek, T. A., and Weinshank, R. L. (1996). A receptor subtype involved in neuropeptide-Y-induced food intake. Nature, 382(6587):168-71.

Germano, C. M. R., de Castro, M., Rorato, R., Laguna, M. T. C., AntunesRodrigues, J., Elias, C. F., and Elias, L. L. K. (2007). Time course effects of adrenalectomy and food intake on cocaine- and amphetamine-regulated transcript expression in the hypothalamus. Brain research, 1166:55-64.

Ginsburg, B. and Allee, W. (1942). Some Effects of Conditioning on Social Dominance and Subordination in Inbred Strains of Mice. Physiological Zoology, 15(4):485-506.

Giordano, R., Pellegrino, M., Picu, A., Bonelli, L., Balbo, M., Berardelli, R., Lanfranco, F., Ghigo, E., and Arvat, E. (2006). Neuroregulation of the hypothalamus-pituitary-adrenal (HPA) axis in humans: effects of GABA, mineralocorticoid-, and GH-Secretagogue-receptor modulation. TheScientific WorldJournal, 6:1-11.

Goebel-Stengel, M., Hofmann, T., Elbelt, U., Teuffel, P., Ahnis, A., Kobelt, P., Lambrecht, N. W. G., Klapp, B. F., and Stengel, A. (2013). The ghrelin activating enzyme ghrelin-O-acyltransferase (GOAT) is present in human plasma and expressed dependent on body mass index. Peptides, 43:13-9.

Goldman, N., Glei, D. A., Seplaki, C., Liu, I.-W., and Weinstein, M. (2005). Perceived stress and physiological dysregulation in older adults. Stress (Amsterdam, Netherlands), 8(2):95-105.

Goldstein, D. S. and McEwen, B. (2002). Allostasis, homeostats, and the nature of stress. Stress (Amsterdam, Netherlands), 5(1):55-8.

Grassi, G. (2007). Adrenergic overdrive as the link among hypertension, obesity, and impaired thermogenesis: lights and shadows. Hypertension, 49(1):5-6.

Green, P. K., Wilkinson, C. W., and Woods, S. C. (1992). Intraventricular corticosterone increases the rate of body weight gain in underweight adrenalectomized rats. Endocrinology, 130(1):269-75.

Greeno, C. G. and Wing, R. R. (1994). Stress-induced eating. Psychological bulletin, 115(3):444-64. 
Griffiths, J., Shanks, N., and Anisman, H. (1992). Strain-specific alterations in consumption of a palatable diet following repeated stressor exposure. Pharmacology, biochemistry, and behavior, 42(2):219-27.

Grill, H. J. and Kaplan, J. M. (2001). Interoceptive and integrative contributions of forebrain and brainstem to energy balance control. International journal of obesity and related metabolic disorders : journal of the International Association for the Study of Obesity, 25 Suppl 5:S73-7.

Grill, H. J. and Kaplan, J. M. (2002). The neuroanatomical axis for control of energy balance. Frontiers in neuroendocrinology, 23(1):2-40.

Groesz, L. M., McCoy, S., Carl, J., Saslow, L., Stewart, J., Adler, N., Laraia, B., and Epel, E. (2012). What is eating you? Stress and the drive to eat. Appetite, 58(2):717-21.

Grosch, J. W., Caruso, C. C., Rosa, R. R., and Sauter, S. L. (2006). Long hours of work in the U.S.: associations with demographic and organizational characteristics, psychosocial working conditions, and health. American journal of industrial medicine, 49(11):943-52.

Grunberg, N. E. and Straub, R. O. (1992). The role of gender and taste class in the effects of stress on eating. Health psychology : official journal of the Division of Health Psychology, American Psychological Association, 11(2):97100.

Guan, X. M., Yu, H., Palyha, O. C., McKee, K. K., Feighner, S. D., Sirinathsinghji, D. J., Smith, R. G., Van der Ploeg, L. H., and Howard, A. D. (1997). Distribution of mRNA encoding the growth hormone secretagogue receptor in brain and peripheral tissues. Brain research. Molecular brain research, 48(1):23-9.

Guarnieri, D. J., Brayton, C. E., Richards, S. M., Maldonado-Aviles, J., Trinko, J. R., Nelson, J., Taylor, J. R., Gourley, S. L., and DiLeone, R. J. (2012). Gene profiling reveals a role for stress hormones in the molecular and behavioral response to food restriction. Biological psychiatry, 71(4):358-65.

Gunapala, K. M., Gallardo, C. M., Hsu, C. T., and Steele, A. D. (2011). Single gene deletions of orexin, leptin, neuropeptide Y, and ghrelin do not appreciably alter food anticipatory activity in mice. PloS one, 6(3):e18377.

Guo, Z.-F., Zheng, X., Qin, Y.-W., Hu, J.-Q., Chen, S.-P., and Zhang, Z. (2007). Circulating preprandial ghrelin to obestatin ratio is increased in human obesity. The Journal of clinical endocrinology and metabolism, 92(5):1875-80.

Gutierrez, J. A., Solenberg, P. J., Perkins, D. R., Willency, J. A., Knierman, M. D., Jin, Z., Witcher, D. R., Luo, S., Onyia, J. E., and Hale, J. E. (2008). Ghrelin octanoylation mediated by an orphan lipid transferase. Proceedings of the National Academy of Sciences of the United States of America, 105(17):6320-5. 
Ha, J., Daniel, S., Broyles, S. S., and Kim, K. H. (1994). Critical phosphorylation sites for acetyl-CoA carboxylase activity. The Journal of biological chemistry, 269(35):22162-8.

Hagan, M. M., Rushing, P. A., Schwartz, M. W., Yagaloff, K. A., Burn, P., Woods, S. C., and Seeley, R. J. (1999). Role of the CNS melanocortin system in the response to overfeeding. The Journal of neuroscience: the official journal of the Society for Neuroscience, 19(6):2362-7.

Halem, H. A., Taylor, J. E., Dong, J. Z., Shen, Y., Datta, R., Abizaid, A., Diano, S., Horvath, T. L., and Culler, M. D. (2005). A novel growth hormone secretagogue-1a receptor antagonist that blocks ghrelin-induced growth hormone secretion but induces increased body weight gain. Neuroendocrinology, 81(5):339-49.

Halford, J. C. G., Harrold, J. A., Boyland, E. J., Lawton, C. L., and Blundell, J. E. (2007). Serotonergic drugs : effects on appetite expression and use for the treatment of obesity. Drugs, 67(1):27-55.

Haller, J., Fuchs, E., Halász, J., and Makara, G. B. (1999). Defeat is a major stressor in males while social instability is stressful mainly in females: towards the development of a social stress model in female rats. Brain research bulletin, $50(1): 33-9$.

Hanukoglu, I., Feuchtwanger, R., and Hanukoglu, A. (1990). Mechanism of corticotropin and cAMP induction of mitochondrial cytochrome P450 system enzymes in adrenal cortex cells. The Journal of biological chemistry, 265(33):20602-8.

Haque, Z., Akbar, N., Yasmin, F., Haleem, M. a., and Haleem, D. J. (2012). Inhibition of immobilization stress-induced anorexia, behavioral deficits, and plasma corticosterone secretion by injected leptin in rats. Stress (Amsterdam, Netherlands), 16(3):353-62.

Hardie, D. G. and Carling, D. (1997). The AMP-activated protein kinase-fuel gauge of the mammalian cell? European journal of biochemistry / FEBS, 246(2):259-73.

Hardie, L. J., Guilhot, N., and Trayhurn, P. (1996). Regulation of leptin production in cultured mature white adipocytes. Hormone and metabolic research $=$ Hormon- und Stoffwechselforschung = Hormones et métabolisme, 28(12):6859.

Hardy, M. P., Sottas, C. M., Ge, R., McKittrick, C. R., Tamashiro, K. L., McEwen, B. S., Haider, S. G., Markham, C. M., Blanchard, R. J., Blanchard, D. C., and Sakai, R. R. (2002). Trends of reproductive hormones in male rats during psychosocial stress: role of glucocorticoid metabolism in behavioral dominance. Biology of reproduction, 67(6):1750-5. 
Harris, R. B., Zhou, J., Youngblood, B. D., Rybkin, I. I., Smagin, G. N., and Ryan, D. H. (1998). Effect of repeated stress on body weight and body composition of rats fed low- and high-fat diets. The American journal of physiology, 275(6 Pt 2):R1928-38.

Havel, P. J. (2001). Peripheral signals conveying metabolic information to the brain: short-term and long-term regulation of food intake and energy homeostasis. Experimental biology and medicine (Maywood, N.J.), 226(11):963-77.

Hayashi, R., Wada, H., Ito, K., and Adcock, I. M. (2004). Effects of glucocorticoids on gene transcription. European journal of pharmacology, 500(1-3):5162 .

Hayes, D. J. and Greenshaw, A. J. (2011). 5-HT receptors and reward-related behaviour: a review. Neuroscience and biobehavioral reviews, 35(6):1419-49.

Haynes, R. C. (1958). The activation of adrenal phosphorylase by the adrenocorticotropic hormone. The Journal of biological chemistry, 233(5):1220-2.

Heisler, L., Cowley, M., Tecott, L., and Fan, W. (2002). Activation of central melanocortin pathways by fenfluramine. Science, 609(2002).

Heisler, L. K., Cowley, M. A., Kishi, T., Tecott, L. H., Fan, W., Low, M. J., Smart, J. L., Rubinstein, M., Tatro, J. B., Zigman, J. M., Cone, R. D., and Elmquist, J. K. (2003). Central serotonin and melanocortin pathways regulating energy homeostasis. Annals of the New York Academy of Sciences, 994:169-74.

Hellerstedt, W. L. and Jeffery, R. W. (1997). The association of job strain and health behaviours in men and women. International journal of epidemiology, 26(3):575-83.

Hennessy, M. B. and Levine, S. (1977). Effects of various habituation procedures on pituitary-adrenal responsiveness in the mouse. Physiology $\mathscr{E}$ behavior, 18(5):799-802.

Herman, J. P. (1993). Regulation of adrenocorticosteroid receptor mRNA expression in the central nervous system. Cellular and Molecular Neurobiology, 13(4):349-372.

Herman, J. P., Cullinan, W. E., Morano, M. I., Akil, H., and Watson, S. J. (1995). Contribution of the Ventral Subiculum to Inhibitory Regulation of the Hypothalamo-Pituitary-Adrenocortical Axis. Journal of Neuroendocrinology, $7(6): 475-482$.

Herman, J. P., Cullinan, W. E., Young, E. A., Akil, H., and Watson, S. J. (1992). Selective forebrain fiber tract lesions implicate ventral hippocampal structures in tonic regulation of paraventricular nucleus corticotropin-releasing hormone $(\mathrm{CRH})$ and arginine vasopressin (AVP) mRNA expression. Brain research, 592(1-2):228-38. 
Herman, J. P., Figueiredo, H., Mueller, N. K., Ulrich-Lai, Y., Ostrander, M. M., Choi, D. C., and Cullinan, W. E. (2003). Central mechanisms of stress integration: hierarchical circuitry controlling hypothalamo-pituitary-adrenocortical responsiveness. Frontiers in neuroendocrinology, 24(3):151-80.

Herman, J. P., Tasker, J. G., Ziegler, D. R., and Cullinan, W. E. (2002). Local circuit regulation of paraventricular nucleus stress integration: glutamateGABA connections. Pharmacology, biochemistry, and behavior, 71(3):457-68.

Herzog, H., Baumgartner, M., Vivero, C., Selbie, L. A., Auer, B., and Shine, J. (1993). Genomic organization, localization, and allelic differences in the gene for the human neuropeptide Y Y1 receptor. The Journal of biological chemistry, 268(9):6703-7.

Heym, J. and Gladfelter, W. E. (1982). Locomotor activity and ingestive behavior after damage to ascending serotonergic systems. Physiology $\&$ behavior, 29(3):459-67.

Hill, J. W. (2012). PVN pathways controlling energy homeostasis. Indian journal of endocrinology and metabolism, 16(Suppl 3):S627-36.

Hoebel, B. G., Hernandez, L., Schwartz, D. H., Mark, G. P., and Hunter, G. A. (1989). Microdialysis studies of brain norepinephrine, serotonin, and dopamine release during ingestive behavior. Theoretical and clinical implications. Annals of the New York Academy of Sciences, 575:171-91; discussion $192-3$.

Hofmann, K. (2000). A superfamily of membrane-bound O-acyltransferases with implications for wnt signaling. Trends in biochemical sciences, 25(3):111-2.

Holst, B., Cygankiewicz, A., Jensen, T. H., Ankersen, M., and Schwartz, T. W. (2003). High constitutive signaling of the ghrelin receptor-identification of a potent inverse agonist. Molecular endocrinology (Baltimore, Md.), 17(11):2201-10.

Holst, B. and Schwartz, T. W. (2004). Constitutive ghrelin receptor activity as a signaling set-point in appetite regulation. Trends in pharmacological sciences, $25(3): 113-7$.

Howard, A. D., Feighner, S. D., Cully, D. F., Arena, J. P., Liberator, P. A., Rosenblum, C. I., Hamelin, M., Hreniuk, D. L., Palyha, O. C., Anderson, J., Paress, P. S., Diaz, C., Chou, M., Liu, K. K., McKee, K. K., Pong, S. S., Chaung, L. Y., Elbrecht, A., Dashkevicz, M., Heavens, R., Rigby, M., Sirinathsinghji, D. J., Dean, D. C., Melillo, D. G., Patchett, A. A., Nargund, R., Griffin, P. R., DeMartino, J. A., Gupta, S. K., Schaeffer, J. M., Smith, R. G., and Van der Ploeg, L. H. (1996). A receptor in pituitary and hypothalamus that functions in growth hormone release. Science (New York, N.Y.), 273(5277):974-7. 
Huhman, K. L. (2006). Social conflict models: can they inform us about human psychopathology? Hormones and behavior, 50(4):640-6.

Hurley, K. M., Herbert, H., Moga, M. M., and Saper, C. B. (1991). Efferent projections of the infralimbic cortex of the rat. The Journal of comparative neurology, 308(2):249-76.

Inhoff, T., Wiedenmann, B., Klapp, B. F., Mönnikes, H., and Kobelt, P. (2009). Is desacyl ghrelin a modulator of food intake? Peptides, 30(5):991-4.

Iwasaki-Sekino, A., Mano-Otagiri, A., Ohata, H., Yamauchi, N., and Shibasaki, T. (2009). Gender differences in corticotropin and corticosterone secretion and corticotropin-releasing factor mRNA expression in the paraventricular nucleus of the hypothalamus and the central nucleus of the amygdala in response to footshock stress or psychological. Psychoneuroendocrinology, 34(2):226-37.

Jacobson, L. (1999). Glucocorticoid replacement, but not corticotropin-releasing hormone deficiency, prevents adrenalectomy-induced anorexia in mice. Endocrinology, 140(1):310-7.

Jahng, J. W., Kim, N. Y., Ryu, V., Yoo, S. B., Kim, B.-T., Kang, D.-W., and Lee, J.-H. (2008). Dexamethasone reduces food intake, weight gain and the hypothalamic 5-HT concentration and increases plasma leptin in rats. European journal of pharmacology, 581(1-2):64-70.

Jankowski, M. P. and Sesack, S. R. (2004). Prefrontal cortical projections to the rat dorsal raphe nucleus: ultrastructural features and associations with serotonin and gamma-aminobutyric acid neurons. The Journal of comparative neurology, 468(4):518-29.

Jerlhag, E. (2008). Systemic administration of ghrelin induces conditioned place preference and stimulates accumbal dopamine. Addiction biology, 13(3-4):35863.

Jerlhag, E., Egecioglu, E., Dickson, S. L., Douhan, A., Svensson, L., and Engel, J. A. (2007). Ghrelin administration into tegmental areas stimulates locomotor activity and increases extracellular concentration of dopamine in the nucleus accumbens. Addiction biology, 12(1):6-16.

Jiang, H., Betancourt, L., and Smith, R. G. (2006). Ghrelin amplifies dopamine signaling by cross talk involving formation of growth hormone secretagogue receptor/dopamine receptor subtype 1 heterodimers. Molecular endocrinology (Baltimore, Md.), 20(8):1772-85.

Jø rgensen, H., Knigge, U., Kjaer, A., Mø ller, M., and Warberg, J. (2002). Serotonergic stimulation of corticotropin-releasing hormone and proopiomelanocortin gene expression. Journal of neuroendocrinology, 14(10):78895 . 
Jø rgensen, H., Knigge, U., Kjaer, A., Vadsholt, T., and Warberg, J. (1998). Serotonergic involvement in stress-induced ACTH release. Brain research, 811(1-2):10-20.

Joibari, M. M. and Khazali, H. (2013). Effect of stress on fasting-induced ghrelin, orexin and galanin secretion in male rats fed different levels of their energy requirement. Obesity (Silver Spring, Md.), 21(1):130-4.

Jones, M. T., Hillhouse, E. W., and Burden, J. L. (1977). Structure-activity relationships of corticosteroid feedback at the hypothalamic level. The Journal of endocrinology, 74(3):415-24.

Kalra, P. S., Dube, M. G., Xu, B., Farmerie, W. G., and Kalra, S. P. (1988). Neuropeptide Y (NPY) Y1 receptor mRNA is upregulated in association with transient hyperphagia and body weight gain: evidence for a hypothalamic site for concurrent development of leptin resistance. Journal of neuroendocrinology, 10(1):43-9.

Kalra, S. P., Dube, M. G., Sahu, A., Phelps, C. P., and Kalra, P. S. (1991). Neuropeptide Y secretion increases in the paraventricular nucleus in association with increased appetite for food. Proceedings of the National Academy of Sciences of the United States of America, 88(23):10931-5.

Kanehisa, M., Akiyoshi, J., Kitaichi, T., Matsushita, H., Tanaka, E., Kodama, K., Hanada, H., and Isogawa, K. (2006). Administration of antisense DNA for ghrelin causes an antidepressant and anxiolytic response in rats. Progress in neuro-psychopharmacology $\& 3$ biological psychiatry, 30(8):1403-7.

Kanoski, S. E., Fortin, S. M., Ricks, K. M., and Grill, H. J. (2013). Ghrelin signaling in the ventral hippocampus stimulates learned and motivational aspects of feeding via PI3K-Akt signaling. Biological psychiatry, 73(9):91523.

Kaur, S., Thankachan, S., Begum, S., Blanco-Centurion, C., Sakurai, T., Yanagisawa, M., and Shiromani, P. J. (2008). Entrainment of temperature and activity rhythms to restricted feeding in orexin knock out mice. Brain research, 1205:47-54.

Kawakami, A. (2008). Leptin inhibits and ghrelin augments hypothalamic noradrenaline release after stress. Stress: The ..., (770885181).

Keeney, A. and Jessop, D. (2006). Differential Effects of Acute and Chronic Social Defeat Stress on HypothalamicâPituitaryâAdrenal Axis Function and Hippocampal Serotonin Release in Mice. Journal of ..., 18(13):330-338.

Kendler, K. S., Gardner, C. O., and Prescott, C. A. (2002). Toward a comprehensive developmental model for major depression in women. The American journal of psychiatry, 159(7):1133-45. 
Kim, K., Arai, K., Sanno, N., Osamura, R. Y., Teramoto, A., and Shibasaki, T. (2001). Ghrelin and growth hormone (GH) secretagogue receptor (GHSR) mRNA expression in human pituitary adenomas. Clinical endocrinology, 54(6):759-68.

King, S. J., Isaacs, a. M., O'Farrell, E., and Abizaid, a. (2011). Motivation to obtain preferred foods is enhanced by ghrelin in the ventral tegmental area. Hormones and behavior, 60(5):572-80.

Kirchner, H., Gutierrez, J. A., Solenberg, P. J., Pfluger, P. T., Czyzyk, T. A., Willency, J. A., Schürmann, A., Joost, H.-g., Jandacek, R. J., Hale, J. E., Heiman, M. L., and Tschöp, M. H. (2009). GOAT links dietary lipids with the endocrine control of energy balance. Nature Medicine, 15(7):741-746.

Kiss, A. and Aguilera, G. (1992). Participation of alpha 1-adrenergic receptors in the secretion of hypothalamic corticotropin-releasing hormone during stress. Neuroendocrinology, 56(2):153-60.

Kiss, J. Z., Martos, J., and Palkovits, M. (1991). Hypothalamic paraventricular nucleus: a quantitative analysis of cytoarchitectonic subdivisions in the rat. The Journal of comparative neurology, 313(4):563-73.

Knigge, K. M. (1961). Adrenocortical Response to Stress in Rats with Lesions in Hippocampus and Amygdala. Experimental Biology and Medicine, 108(1):1821.

Knigge, K. M. and Hays, M. (1963). Evidence of Inhibitive Role of Hippocampus in Neural Regulation of ACTH Release. Experimental Biology and Medicine, 114(1):67-69.

Kobelt, P., Wisser, A.-S., Stengel, A., Goebel, M., Bannert, N., Gourcerol, G., Inhoff, T., Noetzel, S., Wiedenmann, B., Klapp, B. F., Taché, Y., and Mönnikes, H. (2008). Peripheral obestatin has no effect on feeding behavior and brain Fos expression in rodents. Peptides, 29(6):1018-27.

Kohno, D., Gao, H.-Z., Muroya, S., Kikuyama, S., and Yada, T. (2003). Ghrelin directly interacts with neuropeptide-Y-containing neurons in the rat arcuate nucleus: Ca2+ signaling via protein kinase A and N-type channel-dependent mechanisms and cross-talk with leptin and orexin. Diabetes, 52(4):948-56.

Kohno, D., Nakata, M., Maekawa, F., Fujiwara, K., Maejima, Y., Kuramochi, M., Shimazaki, T., Okano, H., Onaka, T., and Yada, T. (2007). Leptin suppresses ghrelin-induced activation of neuropeptide $\mathrm{Y}$ neurons in the arcuate nucleus via phosphatidylinositol 3-kinase- and phosphodiesterase 3-mediated pathway. Endocrinology, 148(5):2251-63.

Kojima, M., Hosoda, H., Date, Y., Nakazato, M., Matsuo, H., and Kangawa, K. (1999). Ghrelin is a growth-hormone-releasing acylated peptide from stomach. Nature, 402(6762):656-60. 
Kojima, M. and Kangawa, K. (2005). Ghrelin: structure and function. Physiological reviews, 85(2):495-522.

Kola, B., Hubina, E., Tucci, S. A., Kirkham, T. C., Garcia, E. A., Mitchell, S. E., Williams, L. M., Hawley, S. A., Hardie, D. G., Grossman, A. B., and Korbonits, M. (2005). Cannabinoids and ghrelin have both central and peripheral metabolic and cardiac effects via AMP-activated protein kinase. The Journal of biological chemistry, 280(26):25196-201.

Konturek, S. J., Konturek, J. W., Pawlik, T., and Brzozowski, T. (2004). Braingut axis and its role in the control of food intake. Journal of physiology and pharmacology : an official journal of the Polish Physiological Society, 55(1 Pt 2):137-54.

Koolhaas, J. M., Meerlo, P., De Boer, S. F., Strubbe, J. H., and Bohus, B. (1997). The temporal dynamics of the stress response. Neuroscience and biobehavioral reviews, 21(6):775-82.

Kramer, R. E., Anderson, C. M., Peterson, J. A., Simpson, E. R., and Waterman, M. R. (1982). Adrenodoxin biosynthesis by bovine adrenal cells in monolayer culture. Induction by adrenocorticotropin. The Journal of biological chemistry, 257(24):14921-5.

Krieger, H. P. and Krieger, D. T. (1970). Chemical stimulation of the brain: effect on adrenal corticoid release. The American journal of physiology, 218(6):1632-41.

Krishnan, V., Han, M., Graham, D., and Berton, O. (2007). Molecular adaptations underlying susceptibility and resistance to social defeat in brain reward regions. Cell, 1:391-404.

Kristensen, P., Judge, M. E., Thim, L., Ribel, U., Christjansen, K. N., Wulff, B. S., Clausen, J. T., Jensen, P. B., Madsen, O. D., Vrang, N., Larsen, P. J., and Hastrup, S. (1998). Hypothalamic CART is a new anorectic peptide regulated by leptin. Nature, 393(6680):72-6.

Kristenssson, E., Sundqvist, M., Astin, M., Kjerling, M., Mattsson, H., Dornonville de la Cour, C., Hå kanson, R., and Lindström, E. (2006). Acute psychological stress raises plasma ghrelin in the rat. Regulatory peptides, 134(2-3):114-7.

Krotkiewski, M., Kral, J. G., and Karlsson, J. (1980). Effects of castration and testosterone substitution on body composition and muscle metabolism in rats. Acta physiologica Scandinavica, 109(3):233-7.

Krukoff, T. L. (1998). Central regulation of autonomic function: no brakes? Clinical and experimental pharmacology $\& 3$ physiology, 25(6):474-8. 
Kumar, J., Chuang, J.-C., Na, E. S., Kuperman, A., Gillman, A. G., Mukherjee, S., Zigman, J. M., McClung, C. A., and Lutter, M. (2013). Differential effects of chronic social stress and fluoxetine on meal patterns in mice. Appetite, 64:81-8.

Kuo, L. and Czarnecka, M. (2008). Chronic stress, combined with a highfat/high-sugar diet, shifts sympatheic signaling toward neuropeptide $\mathrm{Y}$ and leads to obesity and the metabolic syndrome. Annals of the New ..., pages 232-237.

Kuo, L., Kitlinska, J., Tilan, J., and Li, L. (2007). Neuropeptide Y acts directly in the periphery on fat tissue and mediates stress-induced obesity and metabolic syndrome. Nature medicine, 13(7).

Kuperman, Y. and Issler, O. (2010). Perifornical Urocortin-3 mediates the link between stress-induced anxiety and energy homeostasis. Proceedings of the .., 107(18):8393-8398.

Kyrou, I. and Tsigos, C. (2007). Stress mechanisms and metabolic complications. Hormone and metabolic research $=$ Hormon- und Stoffwechselforschung = Hormones et métabolisme, 39(6):430-8.

la Fleur, S. E. (2006). The effects of glucocorticoids on feeding behavior in rats. Physiology \& behavior, 89(1):110-4.

Laitinen, J., Ek, E., and Sovio, U. (2002). Stress-related eating and drinking behavior and body mass index and predictors of this behavior. Preventive medicine, 34(1):29-39.

Lam, T. K. T., Pocai, A., Gutierrez-Juarez, R., Obici, S., Bryan, J., AguilarBryan, L., Schwartz, G. J., and Rossetti, L. (2005). Hypothalamic sensing of circulating fatty acids is required for glucose homeostasis. Nature medicine, $11(3): 320-7$.

Larsen, P. J., Vrang, N., Petersen, P. C., and Kristensen, P. (2000). Chronic intracerebroventricular administration of recombinant CART(42-89) peptide inhibits and causes weight loss in lean and obese Zucker (fa/fa) rats. Obesity research, 8(8):590-6.

Lawrence, C. B., Snape, A. C., Baudoin, F. M.-H., and Luckman, S. M. (2002). Acute central ghrelin and GH secretagogues induce feeding and activate brain appetite centers. Endocrinology, 143(1):155-62.

Le Feuvre, R. A., Aisenthal, L., and Rothwell, N. J. (1991). Involvement of corticotrophin releasing factor $(\mathrm{CRF})$ in the thermogenic and anorexic actions of serotonin (5-HT) and related compounds. Brain research, 555(2):245-50.

Leibowitz, S. F. (1988). Hypothalamic paraventricular nucleus: interaction between alpha 2-noradrenergic system and circulating hormones and nutrients in relation to energy balance. Neuroscience and biobehavioral reviews, 12(2):101-9. 
Leibowitz, S. F., Diaz, S., and Tempel, D. (1989). Norepinephrine in the paraventricular nucleus stimulates corticosterone release. Brain research, 496(12):219-27.

LeSauter, J., Hoque, N., Weintraub, M., Pfaff, D. W., and Silver, R. (2009). Stomach ghrelin-secreting cells as food-entrainable circadian clocks. Proceedings of the National Academy of Sciences of the United States of America, 106(32):13582-7.

Levine, A. and Morley, J. (1981). Stress-induced eating in rats. American Journal of Physiology- ....

Lim, C. T., Kola, B., and Korbonits, M. (2011). The ghrelin/GOAT/GHS-R system and energy metabolism. Reviews in endocrine 83 metabolic disorders, 12(3):173-86.

Lin, L., Saha, P., Ma, X., and Henshaw, I. (2011). Ablation of ghrelin receptor reduces adiposity and improves insulin sensitivity during aging by regulating fat metabolism in white and brown adipose tissues. Aging ....

Liposits, Z., Phelix, C., and Paull, W. K. (1986). Adrenergic innervation of corticotropin releasing factor ( $\mathrm{CRF}$ )-synthesizing neurons in the hypothalamic paraventricular nucleus of the rat. A combined light and electron microscopic immunocytochemical study. Histochemistry, 84(3):201-5.

Liu, J., Garza, J. C., Li, W., and Lu, X.-Y. (2013). Melanocortin-4 receptor in the medial amygdala regulates emotional stress-induced anxiety-like behaviour, anorexia and corticosterone secretion. The international journal of neuropsychopharmacology / official scientific journal of the Collegium Internationale Neuropsychopharmacologicum (CINP), 16(1):105-20.

López, M., Lage, R., Saha, A. K., Pérez-Tilve, D., Vázquez, M. J., Varela, L., Sangiao-Alvarellos, S., Tovar, S., Raghay, K., Rodríguez-Cuenca, S., Deoliveira, R. M., Castañeda, T., Datta, R., Dong, J. Z., Culler, M., Sleeman, M. W., Alvarez, C. V., Gallego, R., Lelliott, C. J., Carling, D., Tschöp, M. H., Diéguez, C., and Vidal-Puig, A. (2008). Hypothalamic fatty acid metabolism mediates the orexigenic action of ghrelin. Cell metabolism, 7(5):389-99.

Lucidi, P., Murdolo, G., Di Loreto, C., Parlanti, N., De Cicco, A., Fatone, C., Taglioni, C., Fanelli, C., Broglio, F., Ghigo, E., Bolli, G. B., Santeusanio, F., and De Feo, P. (2005). Metabolic and endocrine effects of physiological increments in plasma ghrelin concentrations. Nutrition, metabolism, and cardiovascular diseases : NMCD, 15(6):410-7.

Lutter, M., Krishnan, V., Russo, S. J., Jung, S., McClung, C. A., and Nestler, E. J. (2008). Orexin Signaling Mediates the Antidepressant-Like Effect of Calorie Restriction. Journal of Neuroscience, 28(12):3071-3075.

Lutter, M. and Sakata, I. (2008). The orexigenic hormone ghrelin defends against depressive symptoms of chronic stress. Nature . ., 11(7):752-753. 
Mahmoud, S. N., Scaccianoce, S., Scraggs, P. R., Nicholson, S. A., Gillham, B., and Jones, M. T. (1984). Characteristics of corticosteroid inhibition of adrenocorticotrophin release from the anterior pituitary gland of the rat. The Journal of endocrinology, 102(1):33-42.

Makimura, H., Mizuno, T. M., Roberts, J., Silverstein, J., Beasley, J., and Mobbs, C. V. (2000). Adrenalectomy reverses obese phenotype and restores hypothalamic melanocortin tone in leptin-deficient ob/ob mice. Diabetes, 49(11):1917-23.

Malagón, M. M., Luque, R. M., Ruiz-Guerrero, E., Rodríguez-Pacheco, F., García-Navarro, S., Casanueva, F. F., Gracia-Navarro, F., and Castaño, J. P. (2003). Intracellular signaling mechanisms mediating ghrelin-stimulated growth hormone release in somatotropes. Endocrinology, 144(12):5372-80.

Mangos, G. J., Walker, B. R., Kelly, J. J., Lawson, J. A., Webb, D. J., and Whitworth, J. A. (2000). Cortisol inhibits cholinergic vasodilation in the human forearm. American journal of hypertension, 13(11):1155-60.

Maniam, J. and Morris, M. J. (2012). Neuropharmacology The link between stress and feeding behaviour. Neuropharmacology, 63(1):97-110.

Marcus, S. M., Young, E. A., Kerber, K. B., Kornstein, S., Farabaugh, A. H., Mitchell, J., Wisniewski, S. R., Balasubramani, G. K., Trivedi, M. H., and Rush, A. J. (2005). Gender differences in depression: findings from the $\mathrm{STAR}^{*}$ D study. Journal of affective disorders, 87(2-3):141-50.

Margolis, E. B., Lock, H., Hjelmstad, G. O., and Fields, H. L. (2006). The ventral tegmental area revisited: is there an electrophysiological marker for dopaminergic neurons? The Journal of physiology, 577(Pt 3):907-24.

Marín Bivens, C. L., Thomas, W. J., and Stanley, B. G. (1998). Similar feeding patterns are induced by perifornical neuropeptide $\mathrm{Y}$ injection and by food deprivation. Brain research, 782(1-2):271-80.

Márquez, C., Belda, X., and Armario, A. (2002). Post-stress recovery of pituitary-adrenal hormones and glucose, but not the response during exposure to the stressor, is a marker of stress intensity in highly stressful situations. Brain research, 926(1-2):181-5.

Martí, O., Martí, J., and Armario, a. (1994). Effects of chronic stress on food intake in rats: influence of stressor intensity and duration of daily exposure. Physiology $\&$ behavior, 55(4):747-53.

Masuda, Y., Tanaka, T., Inomata, N., Ohnuma, N., Tanaka, S., Itoh, Z., Hosoda, H., Kojima, M., and Kangawa, K. (2000). Ghrelin stimulates gastric acid secretion and motility in rats. Biochemical and biophysical research communications, 276(3):905-8. 
McCormick, C. M., Smith, C., and Mathews, I. Z. (2008). Effects of chronic social stress in adolescence on anxiety and neuroendocrine response to mild stress in male and female rats. Behavioural brain research, 187(2):228-38.

McCune, S. K., Voigt, M. M., and Hill, J. M. (1993). Expression of multiple alpha adrenergic receptor subtype messenger RNAs in the adult rat brain. Neuroscience, 57(1):143-51.

McEwen, B. (2007). Physiology and neurobiology of stress and adaptation: central role of the brain. Physiological reviews, pages 873-904.

McEwen, B. S. (1998). Stress, adaptation, and disease. Allostasis and allostatic load. Annals of the New York Academy of Sciences, 840:33-44.

McEwen, B. S. (2000). Allostasis and allostatic load: implications for neuropsychopharmacology. Neuropsychopharmacology : official publication of the American College of Neuropsychopharmacology, 22(2):108-24.

McEwen, B. S. (2004). Protection and damage from acute and chronic stress: allostasis and allostatic overload and relevance to the pathophysiology of psychiatric disorders. Annals of the New York Academy of Sciences, 1032:1-7.

McEwen, B. S. and Wingfield, J. C. (2010). What is in a name? Integrating homeostasis, allostasis and stress. Hormones and behavior, 57(2):105-11.

McGarry, J. D., Stark, M. J., and Foster, D. W. (1978). Hepatic malonyl-CoA levels of fed, fasted and diabetic rats as measured using a simple radioisotopic assay. The Journal of biological chemistry, 253(22):8291-3.

McKittrick, C. R., Blanchard, D. C., Blanchard, R. J., McEwen, B. S., and Sakai, R. R. (1995). Serotonin receptor binding in a colony model of chronic social stress. Biological psychiatry, 37(6):383-93.

McKittrick, C. R., Magariños, A. M., Blanchard, D. C., Blanchard, R. J., McEwen, B. S., and Sakai, R. R. (2000). Chronic social stress reduces dendritic arbors in CA3 of hippocampus and decreases binding to serotonin transporter sites. Synapse (New York, N.Y.), 36(2):85-94.

McLaughlin, T., Abbasi, F., Lamendola, C., Frayo, R. S., and Cummings, D. E. (2004). Plasma ghrelin concentrations are decreased in insulin-resistant obese adults relative to equally obese insulin-sensitive controls. The Journal of clinical endocrinology and metabolism, 89(4):1630-5.

Merkestein, M., Brans, M. A. D., Luijendijk, M. C. M., de Jong, J. W., Egecioglu, E., Dickson, S. L., and Adan, R. A. H. (2012). Ghrelin mediates anticipation to a palatable meal in rats. Obesity (Silver Spring, Md.), 20(5):963-71.

Michaud, K., Matheson, K., Kelly, O., and Anisman, H. (2008). Impact of stressors in a natural context on release of cortisol in healthy adult humans: a meta-analysis. Stress (Amsterdam, Netherlands), 11(3):177-97. 
Miczek, K. A. (1979). A new test for aggression in rats without aversive stimulation: differential effects of d-amphetamine and cocaine. Psychopharmacology, $60(3): 253-9$.

Miczek, K. A., Thompson, M. L., and Shuster, L. (1982). Opioid-like analgesia in defeated mice. Science (New York, N.Y.), 215(4539):1520-2.

Mieda, M., Williams, S. C., Sinton, C. M., Richardson, J. A., Sakurai, T., and Yanagisawa, M. (2004). Orexin neurons function in an efferent pathway of a food-entrainable circadian oscillator in eliciting food-anticipatory activity and wakefulness. The Journal of neuroscience : the official journal of the Society for Neuroscience, 24(46):10493-501.

Miell, J. P., Englaro, P., and Blum, W. F. (1996). Dexamethasone induces an acute and sustained rise in circulating leptin levels in normal human subjects. Hormone and metabolic research $=$ Hormon- und Stoffwechselforschung = Hormones et métabolisme, 28(12):704-7.

Mineur, Y. S., Belzung, C., and Crusio, W. E. (2006). Effects of unpredictable chronic mild stress on anxiety and depression-like behavior in mice. Behavioural brain research, 175(1):43-50.

Mineur, Y. S., Belzung, C., and Crusio, W. E. (2007). Functional implications of decreases in neurogenesis following chronic mild stress in mice. Neuroscience, $150(2): 251-9$.

Mineur, Y. S., Prasol, D. J., Belzung, C., and Crusio, W. E. (2003). Agonistic behavior and unpredictable chronic mild stress in mice. Behavior genetics, $33(5): 513-9$.

Mitchell, V., Bouret, S., Beauvillain, J. C., Schilling, A., Perret, M., Kordon, C., and Epelbaum, J. (2001). Comparative distribution of mRNA encoding the growth hormone secretagogue-receptor (GHS-R) in Microcebus murinus (Primate, lemurian) and rat forebrain and pituitary. The Journal of comparative neurology, 429(3):469-89.

Mizoguchi, K., Ishige, A., Aburada, M., and Tabira, T. (2003). Chronic stress attenuates glucocorticoid negative feedback: involvement of the prefrontal cortex and hippocampus. Neuroscience, 119(3):887-897.

Moles, a., Bartolomucci, a., Garbugino, L., Conti, R., Caprioli, a., Coccurello, R., Rizzi, R., Ciani, B., and D'Amato, F. R. (2006). Psychosocial stress affects energy balance in mice: modulation by social status. Psychoneuroendocrinology, 31(5):623-33.

Moreau, J. L., Bourson, A., Jenck, F., Martin, J. R., and Mortas, P. (1994). Curative effects of the atypical antidepressant mianserin in the chronic mild stress-induced anhedonia model of depression. Journal of psychiatry $\&$ neuroscience : JPN, 19(1):51-6. 
Mori, K., Kim, J., and Sasaki, K. (2011). Electrophysiological effects of orexin$\mathrm{B}$ and dopamine on rat nucleus accumbens shell neurons in vitro. Peptides, $32(2): 246-52$.

Morilak, D. A., Fornal, C. A., and Jacobs, B. L. (1987a). Effects of physiological manipulations on locus coeruleus neuronal activity in freely moving cats. II. Cardiovascular challenge. Brain research, 422(1):24-31.

Morilak, D. A., Fornal, C. A., and Jacobs, B. L. (1987b). Effects of physiological manipulations on locus coeruleus neuronal activity in freely moving cats. III. Glucoregulatory challenge. Brain research, 422(1):32-9.

Morimoto, M., Morita, N., Ozawa, H., Yokoyama, K., and Kawata, M. (1996). Distribution of glucocorticoid receptor immunoreactivity and mRNA in the rat brain: an immunohistochemical and in situ hybridization study. Neuroscience research, 26(3):235-69.

Morita, S. and Miyata, S. (2012). Different vascular permeability between the sensory and secretory circumventricular organs of adult mouse brain. Cell and tissue research, 349(2):589-603.

Morita, S. and Miyata, S. (2013). Accessibility of low-molecular-mass molecules to the median eminence and arcuate hypothalamic nucleus of adult mouse. Cell biochemistry and function, (October 2012).

Mostyn, A., Keisler, D. H., Webb, R., Stephenson, T., and Symonds, M. E. (2001). The role of leptin in the transition from fetus to neonate. The Proceedings of the Nutrition Society, 60(2):187-94.

Munday, M. R., Campbell, D. G., Carling, D., and Hardie, D. G. (1988). Identification by amino acid sequencing of three major regulatory phosphorylation sites on rat acetyl-CoA carboxylase. European journal of biochemistry / FEBS, 175(2):331-8.

Mundinger, T., Cummings, D., and Jr, G. T. (2006). Direct stimulation of ghrelin secretion by sympathetic nerves. Endocrinology, pages 2893-2901.

Muscat, R. and Willner, P. (1992). Suppression of sucrose drinking by chronic mild unpredictable stress: a methodological analysis. Neuroscience and biobehavioral reviews, 16(4):507-17.

Nakamura, K., Aoike, A., Hosokawa, T., Rokutan, K., Koyama, K., Nishi, Y., Yoshida, A., and Kawai, K. (1990). Effect of food-restriction stress on immune response in mice. Journal of neuroimmunology, 30(1):23-9.

Nakazato, M., Murakami, N., Date, Y., Kojima, M., Matsuo, H., Kangawa, K., and Matsukura, S. (2001). A role for ghrelin in the central regulation of feeding. Nature, 409(6817):194-8. 
Naleid, A. M., Grace, M. K., Cummings, D. E., and Levine, A. S. (2005). Ghrelin induces feeding in the mesolimbic reward pathway between the ventral tegmental area and the nucleus accumbens. Peptides, 26(11):2274-9.

Nieuwenhuizen, A. G. and Rutters, F. (2008). The hypothalamic-pituitaryadrenal-axis in the regulation of energy balance. Physiology $\&$ behavior, 94(2):169-77.

Niswender, K. D. and Schwartz, M. W. (2003). Insulin and leptin revisited: adiposity signals with overlapping physiological and intracellular signaling capabilities. Frontiers in neuroendocrinology, 24(1):1-10.

Nogueiras, R; Lopez, M; Dieguez, C. (2009). Regulation of lipid metabolism by energy availability :. Obesity Reviews, (13):185-201.

Nonogaki, K., Ohashi-Nozue, K., and Oka, Y. (2006). A negative feedback system between brain serotonin systems and plasma active ghrelin levels in mice. Biochemical and biophysical research communications, 341(3):703-7.

Obici, S., Feng, Z., Arduini, A., Conti, R., and Rossetti, L. (2003). Inhibition of hypothalamic carnitine palmitoyltransferase-1 decreases food intake and glucose production. Nature medicine, 9(6):756-61.

Obici, S., Feng, Z., Morgan, K., Stein, D., Karkanias, G., and Rossetti, L. (2002). Central administration of oleic acid inhibits glucose production and food intake. Diabetes, 51(2):271-5.

Ochi, M., Tominaga, K., Tanaka, F., and Tanigawa, T. (2008). Effect of chronic stress on gastric emptying and plasma ghrelin levels in rats. Life sciences, $82: 862-868$.

O'Connor, D. B., Jones, F., Conner, M., McMillan, B., and Ferguson, E. (2008). Effects of daily hassles and eating style on eating behavior. Health psychology : official journal of the Division of Health Psychology, American Psychological Association, 27(1 Suppl):S20-31.

Olijslagers, J. E., de Kloet, E. R., Elgersma, Y., van Woerden, G. M., Joëls, M., and Karst, H. (2008). Rapid changes in hippocampal CA1 pyramidal cell function via pre- as well as postsynaptic membrane mineralocorticoid receptors. The European journal of neuroscience, 27(10):2542-50.

Ollmann, M. M., Wilson, B. D., Yang, Y. K., Kerns, J. A., Chen, Y., Gantz, I., and Barsh, G. S. (1997). Antagonism of central melanocortin receptors in vitro and in vivo by agouti-related protein. Science (New York, N.Y.), 278(5335):135-8.

Olszewski, P. K., Li, D., Grace, M. K., Billington, C. J., Kotz, C. M., and Levine, A. S. (2003). Neural basis of orexigenic effects of ghrelin acting within lateral hypothalamus. Peptides, 24(4):597-602. 
Ottosson, M., Lönnroth, P., Björntorp, P., and Edén, S. (2000). Effects of cortisol and growth hormone on lipolysis in human adipose tissue. The Journal of clinical endocrinology and metabolism, 85(2):799-803.

Pacak, K., Palkovits, M., Kopin, I. J., and Goldstein, D. S. (1995). Stressinduced norepinephrine release in the hypothalamic paraventricular nucleus and pituitary-adrenocortical and sympathoadrenal activity: in vivo microdialysis studies. Frontiers in neuroendocrinology, 16(2):89-150.

Page, M. E., Akaoka, H., Aston-Jones, G., and Valentino, R. J. (1992). Bladder distention activates noradrenergic locus coeruleus neurons by an excitatory amino acid mechanism. Neuroscience, 51(3):555-63.

Palkovits, M. (1973). Isolated removal of hypothalamic or other brain nuclei of the rat. Brain research, 59:449-50.

Palmiter, R. D. (2007). Is dopamine a physiologically relevant mediator of feeding behavior? Trends in neurosciences, 30(8):375-81.

Parker, E., Van Heek, M., and Stamford, A. (2002). Neuropeptide Y receptors as targets for anti-obesity drug development: perspective and current status. European journal of pharmacology, 440(2-3):173-87.

Parker, R. M. and Herzog, H. (1999). Regional distribution of Y-receptor subtype mRNAs in rat brain. The European journal of neuroscience, 11(4):143148 .

Patel, K., Dixit, V. D., Lee, J. H., Kim, J. W., Schaffer, E. M., Nguyen, D., and Taub, D. D. (2012). Identification of ghrelin receptor blocker, D-[Lys3] GHRP-6 as a CXCR4 receptor antagonist. International journal of biological sciences, 8(1):108-17.

Patterson, Z. and Ducharme, R. (2010). Altered metabolic and neurochemical responses to chronic unpredictable stressors in ghrelin receptorâdeficient mice. European Journal of ..., (May):1-8.

Paxinos, G. and Franklin, K. (1997). The Mouse Brain in Stereotaxic Coordinates. Academic Press.

Pearson, R., Gatti, P., and Sahibzada, N. (2007). Ultrastructural evidence for selective noradrenergic innervation of CNS vagal projections to the fundus of the rat. Autonomic ..., 136(202):31-42.

Pecoraro, N., Reyes, F., Gomez, F., Bhargava, A., and Dallman, M. F. (2004). Chronic stress promotes palatable feeding, which reduces signs of stress: feedforward and feedback effects of chronic stress. Endocrinology, 145(8):3754-62.

Peeters, A., Barendregt, J. J., Willekens, F., Mackenbach, J. P., Al Mamun, A., and Bonneux, L. (2003). Obesity in adulthood and its consequences for life expectancy: a life-table analysis. Annals of internal medicine, 138(1):24-32. 
Perello, M., Sakata, I., Birnbaum, S., Chuang, J.-C., Osborne-Lawrence, S., Rovinsky, S. A., Woloszyn, J., Yanagisawa, M., Lutter, M., and Zigman, J. M. (2010). Ghrelin increases the rewarding value of high-fat diet in an orexin-dependent manner. Biological psychiatry, 67(9):880-6.

Pesic, V., Marinkovic, P., Janac, B., Ignjatovic, S., Popic, J., Kanazir, S., and Ruzdijic, S. (2010). Changes of behavioral parameters during long-term food restriction in middle-aged Wistar rats. Physiology $\mathcal{E}$ behavior, 101(5):672-8.

Pfluger, P. T., Kirchner, H., Günnel, S., Schrott, B., Perez-Tilve, D., Fu, S., Benoit, S. C., Horvath, T., Joost, H.-G., Wortley, K. E., Sleeman, M. W., and Tschöp, M. H. (2008). Simultaneous deletion of ghrelin and its receptor increases motor activity and energy expenditure. American journal of physiology. Gastrointestinal and liver physiology, 294(3):G610-8.

Pieribone, V. A., Nicholas, A. P., Dagerlind, A., and Hökfelt, T. (1994). Distribution of alpha 1 adrenoceptors in rat brain revealed by in situ hybridization experiments utilizing subtype-specific probes. The Journal of neuroscience : the official journal of the Society for Neuroscience, 14(7):4252-68.

Pitcher, J. A., Freedman, N. J., and Lefkowitz, R. J. (1998). G protein-coupled receptor kinases. Annual review of biochemistry, 67:653-92.

Plotsky, P. M. (1987). Facilitation of immunoreactive corticotropin-releasing factor secretion into the hypophysial-portal circulation after activation of catecholaminergic pathways or central norepinephrine injection. Endocrinology, 121(3):924-30.

Popper, R., Smits, G., Meiselman, H. L., and Hirsch, E. (1989). Eating in combat: a survey of U.S. Marines. Military medicine, 154(12):619-23.

Pothion, S., Bizot, J.-C., Trovero, F., and Belzung, C. (2004). Strain differences in sucrose preference and in the consequences of unpredictable chronic mild stress. Behavioural brain research, 155(1):135-46.

Pralong, F. P., Corder, R., and Gaillard, R. C. (1993). The effects of chronic glucocorticoid excess, adrenalectomy and stress on neuropeptide $\mathrm{Y}$ in individual rat hypothalamic nuclei. Neuropeptides, 25(4):223-31.

Pratt, W. B., Morishima, Y., Murphy, M., and Harrell, M. (2006). Chaperoning of glucocorticoid receptors. Handbook of experimental pharmacology, (172):111-38.

Rabasa, C., Muñoz Abellán, C., Daviu, N., Nadal, R., and Armario, A. (2011). Repeated exposure to immobilization or two different footshock intensities reveals differential adaptation of the hypothalamic-pituitary-adrenal axis. Physiology $\&$ behavior, 103(2):125-33. 
Raber, J. (1998). Detrimental effects of chronic hypothalamic-pituitary-adrenal axis activation. From obesity to memory deficits. Molecular neurobiology, 18(1):1-22.

Radley, J. (2008). Noradrenergic innervation of the dorsal medial prefrontal cortex modulates hypothalamo-pituitary-adrenal responses to acute emotional stress. The Journal of ..., 28(22):5806-5816.

Räikkönen, K., Keltikangas-Järvinen, L., Adlercreutz, H., and Hautanen, A. (1996). Psychosocial stress and the insulin resistance syndrome. Metabolism: clinical and experimental, 45(12):1533-8.

Ramos, E. J. B., Meguid, M. M., Campos, A. C. L., and Coelho, J. C. U. (2005). Neuropeptide Y, alpha-melanocyte-stimulating hormone, and monoamines in food intake regulation. Nutrition (Burbank, Los Angeles County, Calif.), 21(2):269-79.

Rasmussen, K., Morilak, D. A., and Jacobs, B. L. (1986). Single unit activity of locus coeruleus neurons in the freely moving cat. I. During naturalistic behaviors and in response to simple and complex stimuli. Brain research, $371(2): 324-34$

Raspopow, K., Abizaid, A., Matheson, K., and Anisman, H. (2010). Psychosocial stressor effects on cortisol and ghrelin in emotional and non-emotional eaters: influence of anger and shame. Hormones and behavior, 58(4):677-84.

Razzoli, M. and Domenici, E. (2011). A role for BDNF/TrkB signaling in behavioral and physiological consequences of social defeat stress. Genes, Brain and ..., pages $1-10$.

Rebuffé-Scrive, M., Walsh, U. A., McEwen, B., and Rodin, J. (1992). Effect of chronic stress and exogenous glucocorticoids on regional fat distribution and metabolism. Physiology $\&$ behavior, 52(3):583-90.

Reichenbach, A., Steyn, F. J., Sleeman, M. W., and Andrews, Z. B. (2012). Ghrelin receptor expression and colocalization with anterior pituitary hormones using a GHSR-GFP mouse line. Endocrinology, 153(11):5452-66.

Reimer, R. A., Maurer, A. D., Lau, D. C. W., and Auer, R. N. (2010). Longterm dietary restriction influences plasma ghrelin and GOAT mRNA level in rats. Physiology \& behavior, 99(5):605-10.

Richardson, N. R. and Gratton, A. (1996). Behavior-relevant changes in nucleus accumbens dopamine transmission elicited by food reinforcement: an electrochemical study in rat. The Journal of neuroscience : the official journal of the Society for Neuroscience, 16(24):8160-9.

Riediger, T., Traebert, M., Schmid, H. A., Scheel, C., Lutz, T. A., and Scharrer, E. (2003). Site-specific effects of ghrelin on the neuronal activity in the hypothalamic arcuate nucleus. Neuroscience letters, 341(2):151-5. 
Rizza, R. A., Mandarino, L. J., and Gerich, J. E. (1982). Cortisol-induced insulin resistance in man: impaired suppression of glucose production and stimulation of glucose utilization due to a postreceptor detect of insulin action. The Journal of clinical endocrinology and metabolism, 54(1):131-8.

Robinson, S., Rainwater, A. J., Hnasko, T. S., and Palmiter, R. D. (2007). Viral restoration of dopamine signaling to the dorsal striatum restores instrumental conditioning to dopamine-deficient mice. Psychopharmacology, 191(3):567-78.

Rosmond, R., Dallman, M. F., and Björntorp, P. (1998). Stress-related cortisol secretion in men: relationships with abdominal obesity and endocrine, metabolic and hemodynamic abnormalities. The Journal of clinical endocrinology and metabolism, 83(6):1853-9.

Rowland, N. E. and Antelman, S. M. (1976). Stress-induced hyperphagia and obesity in rats: a possible model for understanding human obesity. Science (New York, N.Y.), 191(4224):310-12.

Rubin, R. T., Mandell, A. J., and Crandall, P. H. (1966). Corticosteroid responses to limbic stimulation in man: localization of stimulus sites. Science (New York, N.Y.), 153(3737):767-8.

Ruis, M. A., te Brake, J. H., Buwalda, B., De Boer, S. F., Meerlo, P., Korte, S. M., Blokhuis, H. J., and Koolhaas, J. M. (1999). Housing familiar male wildtype rats together reduces the long-term adverse behavioural and physiological effects of social defeat. Psychoneuroendocrinology, 24(3):285-300.

Russell, C. D., Petersen, R. N., Rao, S. P., Ricci, M. R., Prasad, A., Zhang, Y., Brolin, R. E., and Fried, S. K. (1998). Leptin expression in adipose tissue from obese humans: depot-specific regulation by insulin and dexamethasone. The American journal of physiology, 275(3 Pt 1):E507-15.

Saad, M. F., Bernaba, B., Hwu, C.-M., Jinagouda, S., Fahmi, S., Kogosov, E., and Boyadjian, R. (2002). Insulin regulates plasma ghrelin concentration. The Journal of clinical endocrinology and metabolism, 87(8):3997-4000.

Saito, M. and Bray, G. A. (1984). Adrenalectomy and food restriction in the genetically obese (ob/ob) mouse. The American journal of physiology, 246(1 Pt 2):R20-5.

Sakata, I., Yang, J., Lee, C. E., Osborne-lawrence, S., Rovinsky, S. A., Elmquist, J. K., Zigman, J. M., Jk, E., and Jm, Z. (2009). Colocalization of ghrelin $\mathrm{O}$-acyltransferase and ghrelin in gastric mucosal cells. American journal of physiology. Endocrinology and metabolism, pages 134-141.

Sangiao-Alvarellos, S., Varela, L., Vázquez, M. J., Da Boit, K., Saha, A. K., Cordido, F., Diéguez, C., and López, M. (2010). Influence of ghrelin and growth hormone deficiency on AMP-activated protein kinase and hypothalamic lipid metabolism. Journal of neuroendocrinology, 22(6):543-56. 
Saphier, D. and Feldman, S. (1989). Adrenoceptor specificity in the central regulation of adrenocortical secretion. Neuropharmacology, 28(11):1231-7.

Sapolsky, R. M., Krey, L. C., and McEwen, B. S. (1984). Glucocorticoidsensitive hippocampal neurons are involved in terminating the adrenocortical stress response. Proceedings of the National Academy of Sciences of the United States of America, 81(19):6174-7.

Sapolsky, R. M., Krey, L. C., and McEwen, B. S. (1986). The neuroendocrinology of stress and aging: the glucocorticoid cascade hypothesis. Endocrine reviews, $7(3): 284-301$.

Sapolsky, R. M., Romero, L. M., and Munck, A. U. (2000). How do glucocorticoids influence stress responses? Integrating permissive, suppressive, stimulatory, and preparative actions. Endocrine reviews, 21(1):55-89.

Savard, P., Mérand, Y., Leblanc, J., and Dupont, A. (1983). Limitation of access to highly palatable foods increases the norepinephrine content of many discrete hypothalamic and amygdaloidal nuclei of rat brain. Life sciences, $33(25): 2513-9$.

Savontaus, E., Conwell, I. M., and Wardlaw, S. L. (2002). Effects of adrenalectomy on AGRP, POMC, NPY and CART gene expression in the basal hypothalamus of fed and fasted rats. Brain research, 958(1):130-8.

Sawchenko, P. E. and Swanson, L. W. (1983). The organization of forebrain afferents to the paraventricular and supraoptic nuclei of the rat. The Journal of comparative neurology, 218(2):121-44.

Schellekens, H., Dinan, T. G., and Cryan, J. F. (2013). Ghrelin at the interface of obesity and reward. Vitamins and hormones, 91:285-323.

Schellekens, H., Finger, B. C., Dinan, T. G., and Cryan, J. F. (2012). Ghrelin signalling and obesity: at the interface of stress, mood and food reward. Pharmacology \&5 therapeutics, 135(3):316-26.

Schmid, D. A., Held, K., Ising, M., Uhr, M., Weikel, J. C., and Steiger, A. (2005). Ghrelin stimulates appetite, imagination of food, GH, ACTH, and cortisol, but does not affect leptin in normal controls. Neuropsychopharmacology : official publication of the American College of Neuropsychopharmacology, 30(6):1187-92.

Schmidt, M. (2009). Chronic social stress during adolescence in mice alters fat distribution in late life: Prevention by antidepressant treatment. Stress: The ..., (770885181).

Schmidt, M. V., Scharf, S. H., Liebl, C., Harbich, D., Mayer, B., Holsboer, F., and Müller, M. B. (2010). Hormones and Behavior A novel chronic social stress paradigm in female mice. Hormones and Behavior, 57(4-5):415-420. 
Schmittgen, T. D. and Livak, K. J. (2008). Analyzing real-time PCR data by the comparative C(T) method. Nature protocols, 3(6):1101-8.

Schwartz, M. W., Woods, S. C., Porte, D., Seeley, R. J., and Baskin, D. G. (2000). Central nervous system control of food intake. Nature, 404(6778):66171.

Sclafani, A., Rinaman, L., Vollmer, R. R., and Amico, J. A. (2007). Oxytocin knockout mice demonstrate enhanced intake of sweet and nonsweet carbohydrate solutions. American journal of physiology. Regulatory, integrative and comparative physiology, 292(5):R1828-33.

Scott, M., Perello, M., Chuang, J., and Sakata, I. (2012). Hindbrain Ghrelin Receptor Signaling Is Sufficient to Maintain Fasting Glucose. PLoS ONE, $7(8): 1-6$.

Seoane, L. M., López, M., Tovar, S., Casanueva, F. F., Señarís, R., and Diéguez, C. (2003). Agouti-related peptide, neuropeptide Y, and somatostatinproducing neurons are targets for ghrelin actions in the rat hypothalamus. Endocrinology, 144(2):544-51.

Sesack, S. R., Deutch, A. Y., Roth, R. H., and Bunney, B. S. (1989). Topographical organization of the efferent projections of the medial prefrontal cortex in the rat: an anterograde tract-tracing study with Phaseolus vulgaris leucoagglutinin. The Journal of comparative neurology, 290(2):213-42.

Sgoifo, A., Koolhaas, J., De Boer, S., Musso, E., Stilli, D., Buwalda, B., and Meerlo, P. (1999). Social stress, autonomic neural activation, and cardiac activity in rats. Neuroscience and biobehavioral reviews, 23(7):915-23.

Shanks, N., Francis, D., Zalcman, S., Meaney, M. J., and Anisman, H. (1994). Alterations in central catecholamines associated with immune responding in adult and aged mice. Brain research, 666(1):77-87.

Shimizu, H., Oh-I, S., Okada, S., and Mori, M. (2009). Nesfatin-1: an overview and future clinical application. Endocrine journal, 56(4):537-43.

Shors, T. J., Mathew, J., Sisti, H. M., Edgecomb, C., Beckoff, S., and Dalla, C. (2007). Neurogenesis and helplessness are mediated by controllability in males but not in females. Biological psychiatry, 62(5):487-95.

Silva, R. C. B., Cruz, A. P. M., Avanzi, V., Landeira-Fernandez, J., and Brandão, M. L. (2002). Distinct contributions of median raphe nucleus to contextual fear conditioning and fear-potentiated startle. Neural plasticity, $9(4): 233-47$.

Simerly, R. (2006). Feeding signals and drugs meet in the midbrain. Nature medicine, 12(11):1244-6. 
Skibicka, K. P., Hansson, C., Alvarez-Crespo, M., Friberg, P. A., and Dickson, S. L. (2011). Ghrelin directly targets the ventral tegmental area to increase food motivation. Neuroscience, 180:129-37.

Smith, S. M. and Vale, W. W. (2006). The role of the hypothalamic-pituitaryadrenal axis in neuroendocrine responses to stress. Dialogues in clinical neuroscience, 8(4):383-95.

Solomon, M. B., Foster, M. T., Bartness, T. J., and Huhman, K. L. (2007). Social defeat and footshock increase body mass and adiposity in male Syrian hamsters. American journal of physiology. Regulatory, integrative and comparative physiology, 292(1):R283-90.

Spencer, S., Xu, L., and Clarke, M. (2012). Ghrelin Regulates the Hypothalamic-Pituitary-Adrenal Axis and Restricts Anxiety After Acute Stress. Biological ....

Stengel, A., Goebel, M., Wang, L., Taché, Y., Sachs, G., and Lambrecht, N. W. G. (2010). Differential distribution of ghrelin-O-acyltransferase (GOAT) immunoreactive cells in the mouse and rat gastric oxyntic mucosa. Biochemical and biophysical research communications, 392(1):67-71.

Stengel, A., Goebel, M., Yakubov, I., Wang, L., Witcher, D., Coskun, T., Taché, Y., Sachs, G., and Lambrecht, N. W. G. (2009). Identification and characterization of nesfatin-1 immunoreactivity in endocrine cell types of the rat gastric oxyntic mucosa. Endocrinology, 150(1):232-8.

Stengel, A., Wang, L., and Taché, Y. (2011). Stress-related alterations of acyl and desacyl ghrelin circulating levels: mechanisms and functional implications. Peptides, 32(11):2208-2217.

Sternson, S. M., Shepherd, G. M. G., and Friedman, J. M. (2005). Topographic mapping of $\mathrm{VMH}-i$ arcuate nucleus microcircuits and their reorganization by fasting. Nature neuroscience, 8(10):1356-63.

Stevanović, D., Milosević, V., Starcević, V. P., and Severs, W. B. (2007). The effect of centrally administered ghrelin on pituitary ACTH cells and circulating ACTH and corticosterone in rats. Life sciences, 80(9):867-72.

Stöhr, T., Szuran, T., Welzl, H., Pliska, V., Feldon, J., and Pryce, C. R. (2000). Lewis/Fischer rat strain differences in endocrine and behavioural responses to environmental challenge. Pharmacology, biochemistry, and behavior, 67(4):809-19.

Stratakis, C. A. and Chrousos, G. P. (1995). Neuroendocrinology and pathophysiology of the stress system. Annals of the New York Academy of Sciences, 771:1-18. 
Sujoldzić, A. and De Lucia, A. (2007). A cross-cultural study of adolescentsBMI, body image and psychological well-being. Collegium antropologicum, 31(1):123-30.

Sun, Y., Asnicar, M., and Smith, R. G. (2007a). Central and peripheral roles of ghrelin on glucose homeostasis. Neuroendocrinology, 86(3):215-28.

Sun, Y., Garcia, J. M., and Smith, R. G. (2007b). Ghrelin and growth hormone secretagogue receptor expression in mice during aging. Endocrinology, 148(3):1323-9.

Svensson, T. H. (1987). Peripheral, autonomic regulation of locus coeruleus noradrenergic neurons in brain: putative implications for psychiatry and psychopharmacology. Psychopharmacology, 92(1):1-7.

Szentirmai, E., Kapás, L., and Krueger, J. M. (2007). Ghrelin microinjection into forebrain sites induces wakefulness and feeding in rats. American journal of physiology. Regulatory, integrative and comparative physiology, 292(1):R575-85.

Takagishi, M. and Chiba, T. (1991). Efferent projections of the infralimbic (area $25)$ region of the medial prefrontal cortex in the rat: an anterograde tracer PHA-L study. Brain research, 566(1-2):26-39.

Tamashiro, K. (2007). Social stress and recovery: implications for body weight and body composition. American Journal of ..., pages 1864-1874.

Tamashiro, K. and Hegeman, M. (2007). Dynamic body weight and body composition changes in response to subordination stress. Physiology $83 \ldots$, 91:440-448.

Tamashiro, K. L. K., Hegeman, M. a., and Sakai, R. R. (2006). Chronic social stress in a changing dietary environment. Physiology $\&$ behavior, 89(4):53642 .

Tamashiro, K. L. K., Nguyen, M. M. N., Fujikawa, T., Xu, T., Yun Ma, L., Woods, S. C., and Sakai, R. R. (2004). Metabolic and endocrine consequences of social stress in a visible burrow system. Physiology $\mathcal{E}^{3}$ behavior, 80(5):68393.

Tannenbaum, B., Tannenbaum, G. S., Sudom, K., and Anisman, H. (2002). Neurochemical and behavioral alterations elicited by a chronic intermittent stressor regimen: implications for allostatic load. Brain research, 953(1-2):8292.

Tassone, F., Broglio, F., Destefanis, S., Rovere, S., Benso, A., Gottero, C., Prodam, F., Rossetto, R., Gauna, C., van der Lely, A. J., Ghigo, E., and Maccario, M. (2003). Neuroendocrine and metabolic effects of acute ghrelin administration in human obesity. The Journal of clinical endocrinology and metabolism, 88(11):5478-83. 
Taylor, S. E., Klein, L. C., Lewis, B. P., Gruenewald, T. L., Gurung, R. A., and Updegraff, J. A. (2000). Biobehavioral responses to stress in females: tend-and-befriend, not fight-or-flight. Psychological review, 107(3):411-29.

Teubner, B. J. W., Garretson, J. T., Hwang, Y., Cole, P. a., and Bartness, T. J. (2013). Inhibition of ghrelin O-acyltransferase attenuates food deprivationinduced increases in ingestive behavior. Hormones and behavior, 63(4):66773.

Theander-Carrillo, C., Wiedmer, P., Cettour-Rose, P., Nogueiras, R., PerezTilve, D., Pfluger, P., Castaneda, T. R., Muzzin, P., Schürmann, A., Szanto, I., Tschöp, M. H., and Rohner-Jeanrenaud, F. (2006). Ghrelin action in the brain controls adipocyte metabolism. The Journal of clinical investigation, 116(7):1983-93.

Thompson, J. L. and Borgland, S. L. (2011). A role for hypocretin/orexin in motivation. Behavioural brain research, 217(2):446-53.

Tomiyama, A. J., Dallman, M. F., and Epel, E. S. (2011). Comfort food is comforting to those most stressed: evidence of the chronic stress response network in high stress women. Psychoneuroendocrinology, 36(10):1513-9.

Torres, S. and Nowson, C. (2007). Relationship between stress, eating behavior, and obesity. Nutrition, 23:887-894.

Toshinai, K., Date, Y., Murakami, N., Shimada, M., Mondal, M. S., Shimbara, T., Guan, J.-L., Wang, Q.-P., Funahashi, H., Sakurai, T., Shioda, S., Matsukura, S., Kangawa, K., and Nakazato, M. (2003). Ghrelin-induced food intake is mediated via the orexin pathway. Endocrinology, 144(4):1506-12.

Treece, B. R., Ritter, R. C., and Burns, G. A. (2000). Lesions of the dorsal vagal complex abolish increases in meal size induced by NMDA receptor blockade. Brain research, 872(1-2):37-43.

Tschöp, M., Smiley, D., and Heiman, M. (2000). Ghrelin induces adiposity in rodents. Nature, 407(October).

Tsujino, N. and Sakurai, T. (2009). Orexin/hypocretin: a neuropeptide at the interface of sleep, energy homeostasis, and reward system. Pharmacological reviews, 61(2):162-76.

Tung, Y. L., Hewson, A. K., and Dickson, S. L. (2004). Glucocorticoiddependent stimulation of adiposity and appetite by a ghrelin mimetic in the rat. European journal of endocrinology / European Federation of Endocrine Societies, 150(6):905-11.

Ulrich-Lai, Y. and Herman, J. (2009). Neural regulation of endocrine and autonomic stress responses. Nature Reviews Neuroscience, 10(juNe). 
Vale, W., Spiess, J., Rivier, C., and Rivier, J. (1981). Characterization of a 41residue ovine hypothalamic peptide that stimulates secretion of corticotropin and beta-endorphin. Science (New York, N.Y.), 213(4514):1394-7.

Valentino, R., Foote, S., and Page, M. (1993). The Locus Coeruleus as a Site for Integrating Corticotropin-Releasing Factor and Noradrenergic Mediation of Stress Responses. ... of the New York Academy ..., 697:173-188.

Vallès, A., Martí, O., García, A., and Armario, A. (2000). Single exposure to stressors causes long-lasting, stress-dependent reduction of food intake in rats. American journal of physiology. Regulatory, integrative and comparative physiology, 279(3):R1138-44.

van den Pol, A. N. (1982). The magnocellular and parvocellular paraventricular nucleus of rat: intrinsic organization. The Journal of comparative neurology, 206(4):317-45.

van der Lely, A. J. (2009). Ghrelin and new metabolic frontiers. Hormone research, 71 Suppl 1:129-33.

Velásquez, D., Martínez, G., and Romero, A. (2011). The central Sirtuin 1/p53 pathway is essential for the orexigenic action of ghrelin. Diabetes.

Velloso, L. A., Araújo, E. P., and de Souza, C. T. (2008). Diet-induced inflammation of the hypothalamus in obesity. Neuroimmunomodulation, 15(3):189-93.

Verhulst, P.-j. and Depoortere, I. (2012). Ghrelin's second life : From appetite stimulator to glucose regulator. 18(25):3183-3195.

Verhulst, P. J., Janssen, S., Tack, J., and Depoortere, I. (2012). Role of the AMP-activated protein kinase (AMPK) signaling pathway in the orexigenic effects of endogenous ghrelin. Regulatory peptides, 173(1-3):27-35.

Vertes, R. P., Fortin, W. J., and Crane, A. M. (1999). Projections of the median raphe nucleus in the rat. The Journal of comparative neurology, 407(4):55582.

Volkow, N. D. and Wise, R. A. (2005). How can drug addiction help us understand obesity? Nature neuroscience, 8(5):555-60.

Wahlestedt, C., Yanaihara, N., and Hå kanson, R. (1986). Evidence for different pre-and post-junctional receptors for neuropeptide $\mathrm{Y}$ and related peptides. Regulatory peptides, 13(3-4):307-18.

Wang, G.-J., Volkow, N. D., and Fowler, J. S. (2002). The role of dopamine in motivation for food in humans: implications for obesity. Expert opinion on therapeutic targets, 6(5):601-9.

Wang, G.-J., Volkow, N. D., Thanos, P. K., and Fowler, J. S. (2004). Similarity between obesity and drug addiction as assessed by neurofunctional imaging: a concept review. Journal of addictive diseases, 23(3):39-53. 
Wang, S.-X., Chen, J.-X., Yue, G.-X., Bai, M.-H., Kou, M.-J., and Jin, Z.-Y. (2012). Xiaoyaosan decoction regulates changes in neuropeptide y and leptin receptor in the rat arcuate nucleus after chronic immobilization stress. Evidence-based complementary and alternative medicine : eCAM, 2012:381278.

Wansink, B., Cheney, M. M., and Chan, N. (2003). Exploring comfort food preferences across age and gender. Physiology 83 behavior, 79(4-5):739-47.

Weaver, J. U. (2008). Classical endocrine diseases causing obesity. Frontiers of hormone research, 36:212-28.

Weaver, J. U., Kopelman, P. G., McLoughlin, L., Forsling, M. L., and Grossman, A. (1993). Hyperactivity of the hypothalamo-pituitary-adrenal axis in obesity: a study of ACTH, AVP, beta-lipotrophin and cortisol responses to insulininduced hypoglycaemia. Clinical endocrinology, 39(3):345-50.

Wellman, P. J. (2000). Norepinephrine and the control of food intake. Nutrition (Burbank, Los Angeles County, Calif.), 16(10):837-42.

Westenbroek, C., Ter Horst, G. J., Roos, M. H., Kuipers, S. D., Trentani, A., and den Boer, J. a. (2003). Gender-specific effects of social housing in rats after chronic mild stress exposure. Progress in neuro-psychopharmacology 83 biological psychiatry, 27(1):21-30.

White, J. D. and Kershaw, M. (1990). Increased hypothalamic neuropeptide Y expression following food deprivation. Molecular and cellular neurosciences, $1(1): 41-8$.

Whitnall, M. H., Kiss, A., and Aguilera, G. (1993). Contrasting effects of central alpha-1-adrenoreceptor activation on stress-responsive and stressnonresponsive subpopulations of corticotropin-releasing hormone neurosecretory cells in the rat. Neuroendocrinology, 58(1):42-8.

Wilding, J. P., Gilbey, S. G., Bailey, C. J., Batt, R. A., Williams, G., Ghatei, M. A., and Bloom, S. R. (1993). Increased neuropeptide-Y messenger ribonucleic acid (mRNA) and decreased neurotensin mRNA in the hypothalamus of the obese (ob/ob) mouse. Endocrinology, 132(5):1939-44.

Willesen, M. G., Kristensen, P., and Rø mer, J. (1999). Co-localization of growth hormone secretagogue receptor and NPY mRNA in the arcuate nucleus of the rat. Neuroendocrinology, 70(5):306-16.

Williams, L. B., Fawcett, R. L., Waechter, A. S., Zhang, P., Kogon, B. E., Jones, R., Inman, M., Huse, J., and Considine, R. V. (2000). Leptin production in adipocytes from morbidly obese subjects: stimulation by dexamethasone, inhibition with troglitazone, and influence of gender. The Journal of clinical endocrinology and metabolism, 85(8):2678-84. 
Willner, P. (1991). Animal models as simulations of depression. Trends in pharmacological sciences, 12(4):131-6.

Willner, P. (1997). Validity, reliability and utility of the chronic mild stress model of depression: a 10-year review and evaluation. Psychopharmacology, 134(4):319-29.

Willner, P., Muscat, R., and Papp, M. (1992). Chronic mild stress-induced anhedonia: a realistic animal model of depression. Neuroscience and biobehavioral reviews, 16(4):525-34.

Willner, P., Towell, A., Sampson, D., Sophokleous, S., and Muscat, R. (1987). Reduction of sucrose preference by chronic unpredictable mild stress, and its restoration by a tricyclic antidepressant. Psychopharmacology, 93(3):358-64.

Wilson, M., Fisher, J., Fischer, A., and Lee, V. (2008). Quantifying food intake in socially housed monkeys : Social status effects on caloric consumption. Physiology \&3 ..., 94:586-594.

Wise, R. A. (2004). Dopamine, learning and motivation. Nature reviews. Neuroscience, 5(6):483-94.

Wise, R. A. (2005). Forebrain substrates of reward and motivation. The Journal of comparative neurology, 493(1):115-21.

Wise, R. A. (2006). The parsing of food reward. American journal of physiology. Regulatory, integrative and comparative physiology, 291(5):R1234-5.

Woodward, D. J., Moises, H. C., Waterhouse, B. D., Yeh, H. H., and Cheun, J. E. (1991). Modulatory actions of norepinephrine on neural circuits. Advances in experimental medicine and biology, 287:193-208.

Wortley, K. E., del Rincon, J.-P., Murray, J. D., Garcia, K., Iida, K., Thorner, M. O., and Sleeman, M. W. (2005). Absence of ghrelin protects against earlyonset obesity. The Journal of clinical investigation, 115(12):3573-8.

Woulfe, J. M., Flumerfelt, B. A., and Hrycyshyn, A. W. (1990). Efferent connections of the A1 noradrenergic cell group: a DBH immunohistochemical and PHA-L anterograde tracing study. Experimental neurology, 109(3):308-22.

Wu, Q., Clark, M. S., and Palmiter, R. D. (2012). Deciphering a neuronal circuit that mediates appetite. Nature, 483(7391):594-7.

Xu, G., Li, Y., An, W., Li, S., Guan, Y., Wang, N., Tang, C., Wang, X., Zhu, Y., Li, X., Mulholland, M. W., and Zhang, W. (2009). Gastric mammalian target of rapamycin signaling regulates ghrelin production and food intake. Endocrinology, 150(8):3637-44.

Yalcin, I., Belzung, C., and Surget, A. (2008). Mouse strain differences in the unpredictable chronic mild stress: a four-antidepressant survey. Behavioural brain research, 193(1):140-3. 
Yamanaka, A., Beuckmann, C. T., Willie, J. T., Hara, J., Tsujino, N., Mieda, M., Tominaga, M., ichi Yagami, K., Sugiyama, F., Goto, K., Yanagisawa, M., and Sakurai, T. (2003). Hypothalamic orexin neurons regulate arousal according to energy balance in mice. Neuron, 38(5):701-13.

Yang, J., Brown, M. S., Liang, G., Grishin, N. V., and Goldstein, J. L. (2008a). Identification of the acyltransferase that octanoylates ghrelin, an appetitestimulating peptide hormone. Cell, 132(3):387-96.

Yang, J., Zhao, T.-J., Goldstein, J. L., and Brown, M. S. (2008b). Inhibition of ghrelin O-acyltransferase (GOAT) by octanoylated pentapeptides. Proceedings of the National Academy of Sciences of the United States of America, 105(31):10750-5.

Young, E. and Korszun, A. (2010). Sex, trauma, stress hormones and depression. Molecular psychiatry, 15(1):23-8.

Zakrzewska, K. E., Cusin, I., Stricker-Krongrad, A., Boss, O., Ricquier, D., Jeanrenaud, B., and Rohner-Jeanrenaud, F. (1999a). Induction of obesity and hyperleptinemia by central glucocorticoid infusion in the rat. Diabetes, 48(2):365-70.

Zakrzewska, K. E., Sainsbury, A., Cusin, I., Rouru, J., Jeanrenaud, B., and Rohner-Jeanrenaud, F. (1999b). Selective dependence of intracerebroventricular neuropeptide Y-elicited effects on central glucocorticoids. Endocrinology, 140(7):3183-7.

Zbucki, R. L., Sawicki, B., Hryniewicz, A., and Winnicka, M. M. (2008). Cannabinoids enhance gastric X/A-like cells activity. Folia histochemica et cytobiologica / Polish Academy of Sciences, Polish Histochemical and Cytochemical Society, 46(2):219-24.

Zhang, J. V., Ren, P.-G., Avsian-Kretchmer, O., Luo, C.-W., Rauch, R., Klein, C., and Hsueh, A. J. W. (2005). Obestatin, a peptide encoded by the ghrelin gene, opposes ghrelin's effects on food intake. Science (New York, N.Y.), 310(5750):996-9.

Zhao, T.-J., Sakata, I., Li, R. L., Liang, G., Richardson, J. A., Brown, M. S., Goldstein, J. L., and Zigman, J. M. (2010). Ghrelin secretion stimulated by \{beta\}1-adrenergic receptors in cultured ghrelinoma cells and in fasted mice. Proceedings of the National Academy of Sciences of the United States of America, 107(36):15868-73.

Zheng, H. and Berthoud, H. (2008). Neural systems controlling the drive to eat: mind versus metabolism. Physiology, pages 75-83.

Zheng, J., Dobner, A., Babygirija, R., Ludwig, K., and Takahashi, T. (2009). Effects of repeated restraint stress on gastric motility in rats. American journal of physiology. Regulatory, integrative and comparative physiology, 296(5):R1358-65. 
Zhou, L., Williams, T., Lachey, J. L., Kishi, T., Cowley, M. A., and Heisler, L. K. (2005). Serotonergic pathways converge upon central melanocortin systems to regulate energy balance. Peptides, 26(10):1728-32.

Zigman, J. and Jones, J. (2006). Expression of ghrelin receptor mRNA in the rat and the mouse brain. The Journal of .., 548(August 2005):528-548.

Zuber, M. X., Simpson, E. R., Hall, P. F., and Waterman, M. R. (1985). Effects of adrenocorticotropin on 17 alpha-hydroxylase activity and cytochrome P-450(17 alpha) synthesis in bovine adrenocortical cells. The Journal of biological chemistry, 260(3):1842-8. 